\title{
Uniform homeomorphisms between unit spheres of interpolation spaces
}

\author{
Rafaela Gesing
}

\author{
Dissertation SubmitTed \\ TO THE \\ Institute of Mathematics And Statistics \\ OF THE \\ University of SÃO PAUlO \\ FOR \\ THE DEGREE \\ OF \\ MASTER OF SCIENCE \\ Program: Mathematics \\ Advisor: Prof. Dr. Valentin Raphael Henri Ferenczi
}

During the development of this dissertation the author was financially supported by FAPESP Grants 2017/14848-2 and 2018/08362-2

São Paulo, July 2020 


\section{Uniform homeomorphisms between unit spheres of interpolation spaces}

Esta versão da dissertação contém as correções e alterações sugeridas pela Comissão Julgadora durante a defesa da versão original do trabalho, realizada em 20/07/2020. Uma cópia da versão original está disponível no

Instituto de Matemática e Estatística da Universidade de São Paulo.

Comissão Julgadora:

- Prof. Dr. Valentin Raphael Henri Ferenczi - IME-USP

- Prof. Dr. Pedro Tradacete - ICMAT

- Prof. Dr. Willian Hans Goes Corrêa - ICMC-USP 


\section{Acknowledgements}

I would first like to thank my advisor Valentin Ferenczi for all the support, help and guidance during this process. I also want to thank Pedro Tradacete for welcoming me during my time in Spain, and for all the help and advice.

To my friends, thank you for being with me, and for making this process undoubtedly more pleasant. To my mathematical colleagues: your support, help and collaboration certainly made me grow immeasurably.

Finally, I would like to thank the members of the Judging Committee for the helpful comments, and FAPESP for the support that made possible the development of this project. 



\section{Resumo}

GESING, R. Homeomorfismos uniformes entre esferas unitárias de espaços de interpolação. 2020. 100 f. Dissertação (Mestrado) - Instituto de Matemática e Estatística, Universidade de São Paulo, São Paulo, 2020.

Esta dissertação tem por objetivo apresentar um estudo detalhado do artigo Homéomorphismes uniformes entre les sphères unité des espaces d'interpolation de M. Daher (1995), onde ele mostra que, sob certas hipóteses, as esferas unitárias de dois espaços de interpolação complexa são uniformemente homeomorfas. Com esse intuito em mente, conceitos essenciais serão abordados, entre eles, primeiramente, a teoria em que os resultados investigados são desenvolvidos: teoria de espaços uniformemente convexos, integral de Bochner, Método Complexo de Interpolação de A. Calderón. Em segundo lugar, apresentamos aplicações do estudo de homeomorfismos uniformes entre esferas unitárias da escala de interpolação de espaços de Banach, incluindo os espaços $L_{p}$ e os espaços $L_{p}$ com peso. Finalmente, apresentamos alguns tópicos sobre a teoria de reticulados de Banach e sua interação com a teoria de interpolação, apresentando a construção de Calderón-Lozanovskii e o homeomorfismo uniforme entre as esferas unitárias nesse cenário.

Palavras-chave: Interpolação complexa, homeomorfismos uniformes entre esferas, reticulados de Banach. 



\section{Abstract}

GESING, R. Uniform homeomorphisms between unit spheres of interpolation spaces. 2020. 100 p. Dissertation (Master) - Instituto de Matemática e Estatística, Universidade de São Paulo, São Paulo, 2020.

This dissertation aims to present a detailed study of the article Homéomorphismes uniformes entre les sphères unité des espaces d'interpolation by M. Daher (1995), where he shows that, under certain hypotheses, the unit spheres of two complex interpolation spaces are uniformly homeomorphic. With this goal in mind, essential concepts will be addressed, among them, first, the theory where the results investigated are developed: theory of uniformly convex spaces, Bochner integral, and the Complex Interpolation Method of A. Calderón. Following, we present applications on the study of uniform homeomorphisms between unit spheres of Banach spaces on the interpolation scale, including the context of $L_{p}$ spaces and weighted $L_{p}$ spaces. Finally, we introduce some topics on the theory of Banach lattices and its interplay with interpolation theory, presenting the Calderón-Lozanovskii construction and the uniform homeomorphism between unit spheres in this setting.

Keywords: Complex Interpolation, Uniform homeomorphisms between spheres, Banach lattices. 



\section{Contents}

$\begin{array}{ll}\text { Introduction } & 1\end{array}$

1 Preliminary Results 5

1.1 Results in Functional Analysis . . . . . . . . . . . . . . . . . . . . . 5

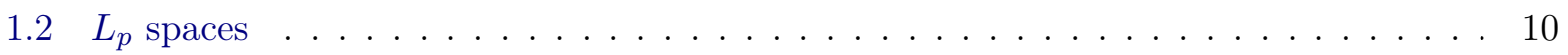

1.3 Hardy Spaces . . . . . . . . . . . . . . . . . . . . . . . . . 15

1.4 Holomorphic Functions in Banach Spaces . . . . . . . . . . . . . . . 17

1.5 Bochner Integral . . . . . . . . . . . . . . . . . . . . . . . . . 21

2 The Complex Method of Interpolation $\quad 29$

2.1 Riesz-Thorin Interpolation Theorem . . . . . . . . . . . . . . . . . . . 30

2.2 Interpolation Spaces . . . . . . . . . . . . . . . . . . . . . . 33

2.3 The Complex Method of Interpolation . . . . . . . . . . . . . . . . . 37

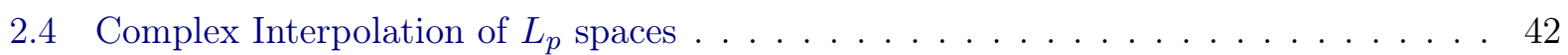

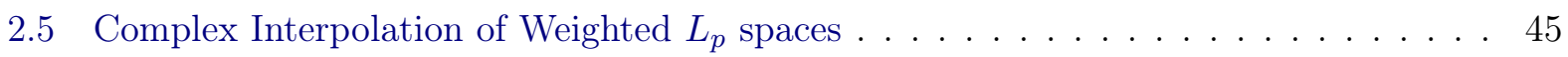

2.6 Notes and Comments . . . . . . . . . . . . . . . . . . . . . 50

3 Uniform homeomorphisms between unit spheres of interpolation spaces $\quad 51$

3.1 Definitions and Notations . . . . . . . . . . . . . . . . . . 51

3.2 Uniform homeomorphism between unit spheres . . . . . . . . . . . . . . . . 59

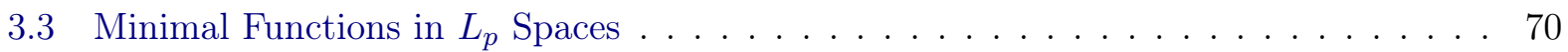

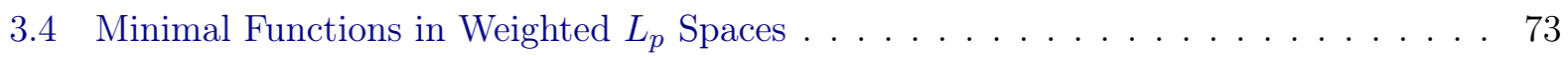

3.5 Vertical and Horizontal Maps . . . . . . . . . . . . . . . . . . . . . 77

3.6 Notes and Comments . . . . . . . . . . . . . . . . . . . . 81

4 Banach Lattices $\quad 83$

4.1 Initial Definitions . . . . . . . . . . . . . . . . . . . . . 83

4.2 Construction of Banach lattices . . . . . . . . . . . . . . . . . . . . 84

4.2 .1 Calderón-Lozanovskii Construction . . . . . . . . . . . . . . . . . . . 87

4.3 Banach Lattices and Uniform Homeomorphisms . . . . . . . . . . . . . . . . . 91

5 Further discussion $\quad 95$

$\begin{array}{ll}\text { Bibliography } & 97\end{array}$

$\begin{array}{lr}\text { Index } & 100\end{array}$ 



\section{Introduction}

Banach spaces fall into the class of vector spaces that have a norm and, therefore, a topology. Thus, when considering their classification, we can categorize them through their linear structure and, in this sense, bounded linear operators represent the translation of characteristics from one space to another. As for the topological structure, such classification occurs through homeomorphisms. An intermediate notion that can be considered is the uniform structure and, in this regard, we can classify Banach spaces through uniform homeomorphisms.

Let us analyze the interplay between these different notions. Note that if two Banach spaces are linearly isomorphic, this automatically implies that they are uniformly homeomorphic (since any bounded linear application is uniformly continuous). The question then remains of the validity of the converse:

If two spaces are uniformly homeomorphic, are they linearly isomorphic? $(*)$

The answer is negative when we consider only homeomorphisms, not necessarily uniform: M. I. Kadec [Kad67] proved that all separable infinite-dimensional Banach spaces are mutually homeomorphic (thus, for $1 \leq p, q<\infty, L_{p}$ and $L_{q}$ are homeomorphic, but it is well-known that they are not linearly isomorphic for $p \neq q$ ). In addition, if two Banach spaces are homeomorphic, they are not necessarily uniformly homeomorphic: the results of J. Lindenstrauss [Lin64] and P. Enflo [Enf70a] show us that for $1 \leq p<q<\infty, L_{p}$ is not uniformly homeomorphic to $L_{q}$.

Going in the direction of answering the question $(*)$, we have the result of P. Enflo [Enf70b] which states that if a Banach space is uniformly homeomorphic to a Hilbert space, then it is isomorphic to the Hilbert space. A more recent result given by W. B. Johnson, J. Lindenstrauss and G. Schechtman [JLS96] shows that if a Banach space is uniformly homeomorphic to some $\ell_{p}, 1<p<\infty$, then it is linearly isomorphic to the same space.

The negative answer to the question $(*)$ was presented by M. Ribe [Rib84], where he shows an example of two separable uniformly homeomorphic Banach spaces which are not linearly isomorphic. Later on, using the techniques presented by M. Ribe, I. Aharoni and J. Lindenstrauss [AL85], W. B. Johnson, J. Lindenstrauss and G. Schechtman [JLS96], and N. J. Kalton [Kal13] exhibited more examples of spaces that are uniformly homeomorphic, but not linearly isomorphic.

One of the classical results concerning the topology of infinite-dimensional Banach spaces was given by S. Mazur [Maz29], where he proved that the unit spheres of two $L_{p}$ spaces, for $1 \leq p<\infty$, 
are uniformly homeomorphic, giving an explicit mapping that we call the Mazur Map. Thus, if two spaces have their unit spheres uniformly homeomorphic, the spaces are not necessarily uniformly homeomorphic. Therefore, it becomes interesting to classify Banach spaces through uniform homeomorphisms between their spheres.

Our study will focus on presenting in detail a result that sheds light on the theory of classification of Banach spaces through the uniform structure of their spheres: under certain hypotheses, the unit spheres of two interpolation spaces are uniformly homeomorphic. This result was presented by M. Daher in [Dah95].

The interpolation spaces that we consider here are obtained through the Complex Interpolation Method, introduced by A. Calderón in [Cal64]. Given a couple $\left(A_{0}, A_{1}\right)$ of Banach spaces that are embedded in a common space and $0<\theta<1$, the complex interpolation method defines the Banach space $A_{\theta}=\left(A_{0}, A_{1}\right)_{\theta}$ in the following way: let $\mathbb{S}=\{z \in \mathbb{C}: 0<\mathfrak{R} z<1\}$ and let $\mathcal{F}(\bar{A})$ be the space of holomorphic bounded functions $f: \mathbb{S} \longrightarrow A_{0}+A_{1}$ such that $f$ has a continuous extension to the closure $\overline{\mathbb{S}}$ and the functions $t \longmapsto f(j+i t)$ are continuous and bounded on $A_{j}$ for $j=0,1$. The space $\mathcal{F}(\bar{A})$ is a Banach space if we consider the norm:

$$
\|f\|_{\mathcal{F}(\bar{A})}=\max \left\{\sup _{t \in \mathbb{R}}\|f(i t)\|_{A_{0}}, \sup _{t \in \mathbb{R}}\|f(1+i t)\|_{A_{1}}\right\} .
$$

We define $A_{\theta}=\left(A_{0}, A_{1}\right)_{\theta}$ as the interpolation space by the following relation: $a \in A_{\theta}$ if, and only if, there is $f \in \mathcal{F}(\bar{A})$ such that $f(\theta)=a$. The norm in $A_{\theta}$ is given by

$$
\|a\|_{A_{\theta}}=\inf \left\{\|f\|_{\mathcal{F}(\bar{A})}: f \in \mathcal{F}(\bar{A}), f(\theta)=a\right\} .
$$

With the notation above, the result presented by Daher in [Dah95] is as follows: if $\left(A_{0}, A_{1}\right)$ is an interpolation couple, $0<\theta_{1}, \theta_{2}<1$ and $A_{0}$ and $A_{1}$ are uniformly convex, then there is a uniform homeomorphism between the unit sphere of the complex interpolation space $A_{\theta_{1}}$ and the unit sphere of $A_{\theta_{2}}$.

In Chapter 1 we gather preliminary results that will be useful throughout the text. As we saw above, the $L_{p}$ spaces provide a wide range of examples in different contexts. It will be no different for the theory developed here, we will use these spaces extensively to illustrate the concepts presented. Section 1.2 assemble some results regarding Lebesgue spaces.

The article by Daher [Dah95] relies on a collection of classical theories in the context of Banach spaces. In Sections 1.4 and 1.5 we study those generalizations:

1. Holomorphic functions in Banach Spaces: The classical theory of complex functions was extended to a setting where the functions take values in a Banach space and holomorphic functions in this context satisfy a "weak property".

2. Bochner integral: We can generalize the theory of Lebesgue to, once more, a setting where functions are taking values in a Banach space. If we consider the integral of a holomorphic function in this setting we can see that many important results are valid here, e.g. the Cauchy Integral Formula. 
Chapter 2 is devoted to the study of the complex interpolation method. Section 2.1 presents one motivation for the complex method, the Riesz-Thorin Interpolation Theorem. The intuition behind a interpolation space is of being an intermediate space between two other spaces; Section 2.2 focuses on formalizing those intuitive ideas.

In the section 2.3 we present the description of the method, along with some properties. The last sections of Chapter 2 present examples of the use of the complex interpolation method.

Chapter 3 is dedicated to the proof of the main result obtained by Daher. We will see that we can define the complex interpolation space through the holomorphic functions over $\mathbb{S}$ however replacing the continuity of the operators over $\overline{\mathbb{S}}$ by a condition of $L_{p}$ integrability. This allows us to find a function that reaches the infimum in equation (1), this minimal representation is what we call minimal function, and plays an essential role in obtaining uniform homeomorphism between the spheres of the interpolation spaces. In the last sections of Chapter 3 we apply Daher's theory to the $L_{p}$ and weighted $L_{p}$ spaces, finding the minimal functions associated to these interpolation scales. Lastly, we investigate some symmetries of the theory by comparing the horizontal maps that arise at different levels of the interpolation scale.

Chapter 4 is dedicated to the theory of Banach lattices and its interplay with the complex interpolation method. We present the Calderón-Lozanovskii Construction and the minimal function associated with the Banach lattice interpolation scale.

Finally, we include further discussion on the topics presented here. We use standard notation and terminology. The reader is expected to be familiar with basic concepts in complex analysis, functional analysis and measure theory. 



\section{Chapter 1}

\section{Preliminary Results}

In this section, we will present preliminary results that will be useful throughout the text and we will fix some notations. We begin by presenting some results of functional analysis, $L_{p}$ spaces and Hardy spaces; we also present the underlying theory needed to understand Daher's statements in [Dah95]: the theory of holomorphic functions that take values in Banach spaces and integration of Banach valued functions.

\subsection{Results in Functional Analysis}

In this section, we recall some classical results and definitions in Functional Analysis with the purpose of fixing notations and ensuring the appropriate versions of classical theorems that we will need. The main references used in this section are [Meg12] and $\left[\mathrm{FHH}^{+} 01\right]$.

Let $X$ be a normed space. We denote by $B\left(x_{0}, r\right)$ the open ball centered at $x_{0} \in X$ with radius $r$, i.e., $B\left(x_{0}, r\right)=\left\{x \in X:\left\|x-x_{0}\right\|_{X}<r\right\}$. We consider $B_{X}=B(0,1)$ (unit ball centered at the origin). $S_{X}$ will denote the unit sphere centered at the origin.

The following lemma is classically used to demonstrate the Open Mapping Theorem.

Lemma 1.1.1. Let $X$ be a Banach space, $\mathrm{Y}$ a normed space, and $T \in \mathcal{B}(X, Y)$. If $r, s>0$ satisfy $B(0, s) \subset \overline{T(B(0, r))}$, then $B(0, s) \subset T(B(0, r))$.

Proof. See [FHH $\left.{ }^{+} 01\right]$, Lemma 2.23.

The following lemma will be useful later on:

Lemma 1.1.2. Let $\left(X,\|\cdot\|_{1}\right)$ be a Banach space, $Y$ a subspace of $X$, and $\|\cdot\|_{2}$ a norm in $Y$ such that $\left(Y,\|\cdot\|_{2}\right)$ is a Banach space. Suppose that $\|x\|_{1} \leq\|x\|_{2}$ for all $x \in Y$ and $B_{Y}=\left\{y \in Y:\|y\|_{2} \leq 1\right\}$ is dense in $B_{X}=\left\{x \in X:\|x\|_{1} \leq 1\right\}$, then $X=Y$ and $\|\cdot\|_{1}=\|\cdot\|_{2}$.

Proof. Consider the identity $I d:\left(Y,\|\cdot\|_{2}\right) \longrightarrow\left(X,\|\cdot\|_{1}\right)$. Since $\|x\|_{1} \leq\|x\|_{2}$ for every $x \in Y$, we have that $I d$ is continuous. Let $B_{1}=\left\{y \in Y:\|y\|_{1}<1\right\}$ and $B_{2}=\left\{y \in Y:\|y\|_{2}<1\right\}$. Note that $B_{2} \subseteq B_{1}$. 
Let us show that $B_{1} \subseteq B_{2}$. Let $\epsilon>0$ and $y \in B_{1} \subseteq B_{X}$. By hypothesis we have $\bar{B}_{Y}^{\|\cdot\|_{1}}=B_{X}$, so there is $z \in B_{Y}$ such that $\|z-y\|_{1}<\frac{\epsilon}{2}$. Moreover, since $\overline{B_{2}}\|\cdot\|_{2}=B_{Y}$, there is $w \in B_{2}$ such that $\|z-w\|_{2}<\frac{\epsilon}{2}$. Thus

$$
\|y-w\|_{1} \leq\|y-z\|_{1}+\|z-w\|_{1} \leq\|y-z\|_{1}+\|z-w\|_{2}<\frac{\epsilon}{2}+\frac{\epsilon}{2}=\epsilon .
$$

Therefore, $y \in{\overline{B_{2}}}^{\|\cdot\|_{1}}$, i.e. $B_{1} \subseteq{\overline{B_{2}}}^{\|\cdot\|_{1}}={\overline{\operatorname{Id(}\left(B_{2}\right)}}^{\|\cdot\|_{1}}$. It follows, then, from Lemma 1.1.1 that $B_{1} \subseteq$ $\operatorname{Id}\left(B_{2}\right)=B_{2}$. Thus, $B_{1}=B_{2}$.

Now let $y \in Y$. Let us show that $\|y\|_{1}=\|y\|_{2}$. For each $n \in \mathbb{N}$, let $x_{n} \doteq \frac{y}{\|y\|_{1}+\frac{1}{n}}$. Note that $\left\|x_{n}\right\|_{1}=$ $\frac{\|y\|_{1}}{\|y\|_{1}+\frac{1}{n}}<1, \forall n \in \mathbb{N}$, i.e. $x_{n} \in B_{1}=B_{2}$. Thus, for all $n \in \mathbb{N},\|y\|_{2}<\|y\|_{1}+\frac{1}{n}$ which implies that $\|y\|_{2} \leq\|y\|_{1}$. Along with the hypothesis, we therefore have $\|y\|_{1}=\|y\|_{2}, \forall y \in Y$.

Since $\left(Y,\|\cdot\|_{2}\right)$ is Banach and $\|\cdot\|_{1}=\|\cdot\|_{2}$ in $Y$, we have that $\left(Y,\|\cdot\|_{1}\right)$ is Banach, and therefore closed. In addition, $B_{X} \cap Y=\left\{x \in Y:\|x\|_{1} \leq 1\right\}=\left\{x \in Y:\|x\|_{2} \leq 1\right\}=B_{Y}$. Thus, $B_{X}=\overline{B_{Y}}\|\|_{1}=\bar{B} X \cap^{\|\cdot\|_{1}} \subseteq$ $\bar{B}_{X}\|\cdot\|_{1} \cap \bar{Y}^{\|\cdot\|_{1}}=B_{X} \cap Y$, which implies that $B_{X} \subseteq Y$. Finally, if $y \in Y \backslash\{0\}$, then $\frac{y}{\|y\|_{1}} \in B_{X}$, and $y \in X$. Hence, $X=Y$ and $\|\cdot\|_{1}=\|\cdot\|_{2}$.

The Hahn-Banach Theorem is an essential tool in functional analysis. We can say, in fact, that the Hahn-Banach Theorem refers to a class of operator extension theorems, or to a class of separation theorems. We will now present the statement of the Hahn-Banach Theorem which concerns the extension of linear functionals, as well as some of its important corollaries.

Theorem 1.1.3. (Hahn-Banach Theorem) Let $X$ be a normed space and $Y$ a subspace of $X$. If $y^{*} \in Y^{*}$, then there is $x^{*} \in X^{*}$ such that $y^{*}(y)=x^{*}(x)$ for all $y \in Y$ and $\left\|y^{*}\right\|_{Y^{*}}=\left\|x^{*}\right\|_{X^{*}}$.

Proof. See [Meg12], Theorem 1.9.6.

Corollary 1.1.4. Let $X$ be a normed space and $0 \neq x \in X$. Then there is $x^{*} \in X^{*}$ such that $\left\|x^{*}\right\|_{X^{*}}=1$ and $x^{*}(x)=\|x\|_{X}$.

Proof. See [Meg12], Corollary 1.9.8.

Corollary 1.1.5. Let $X$ be a normed space and $x, y \in X$ such that $x \neq y$. Then there is $0 \neq x^{*} \in X^{*}$ such that $x^{*}(x) \neq x^{*}(y)$.

Proof. See [Meg12], Corollary 1.9.9.

Definition 1.1.6. Let $X$ be a normed space. For each $x \in X$ and $x^{*} \in X^{*}$, let $I(x)\left(x^{*}\right)=x^{*} x$. Note that $I$ is an isometric isomorphism from $X$ into $I(X) \subseteq X^{* *} . I$ is called the natural map from $X$ into $X^{* *}$. The normed space $X$ is called reflexive if $I(X)=X^{* *}$.

Proposition 1.1.7. Consider $X$ a reflexive space. Then: 
(a) Every closed subspace of $X$ is reflexive.

(b) If $Y$ is isomorphic to $X$, then $Y$ is reflexive.

Proof. See [Meg12].

Proposition 1.1.8. A Banach space $X$ is reflexive if and only if for each closed convex nonempty subset $A$ of $X$, there is at least one $x \in A$ such that $\|x\|_{X}=\mathrm{d}(0, A)$.

Proof. See [Phe60], page 253.

The theory of uniformly convex spaces will be essential for the main result presented here.

Definition 1.1.9. A normed space $X$ is strictly convex if $\|t x+(1-t) y\|_{X}<1$ for all $t \in(0,1)$ and for all $x, y \in S_{X}$, with $x \neq y$.

Proposition 1.1.10. Consider $X$ a strictly convex normed space and $x, y \in X$. Then $\|x+y\| \leq$ $\|x\|+\|y\|$, with equality if and only if one of the two vectors is a nonnegative real multiple of the other.

Proof. See [Meg12], Corollary 5.1.12.

Let us consider some characterizations of strictly convex spaces:

Proposition 1.1.11. Let $X$ be a normed space and $1<p<\infty$. We have:

(a) $X$ is strictly convex if and only if, for all $x, y \in X$ such that $x \neq y$, we have

$$
\left\|\frac{x+y}{2}\right\|_{X}^{p}<\frac{\|x\|_{X}^{p}+\|y\|_{X}^{p}}{2} .
$$

(b) $X$ is strictly convex if and only if, for all $x, y \in X$ such that $x \neq y,\|x\|_{X}=\|y\|_{X}=1$ we have

$$
\left\|\frac{x+y}{2}\right\|_{X}<1
$$

Proof. See [Bea11], Proposition 1 in Part 3, Chapter 1, Section 1.

Definition 1.1.12. Let $(X,\|\cdot\|)$ be a normed space and $\epsilon \in(0,2]$. We define the modulus of convexity of the norm $\|\cdot\|$ (or the normed space) by

$$
\delta_{X}(\epsilon)=\inf \left\{1-\left\|\frac{x+y}{2}\right\|: x, y \in B_{X},\|x-y\| \geq \epsilon\right\} .
$$


The norm $\|\cdot\|$ is called uniformly convex if $\delta_{X}(\epsilon)>0$ for all $\epsilon \in(0,2]$. The space $(X,\|\cdot\|)$ is then called a uniformly convex space.

In the following proposition we give some properties of uniformly convex spaces:

Proposition 1.1.13. Consider $X$ a uniformly convex space. Then:

(a) $X$ is strictly convex.

(b) $X$ is reflexive.

(c) Every closed subspace of $X$ is uniformly convex.

(d) If $Y$ is isometric to $X$, then $Y$ is uniformly convex.

Proof. See [Meg12].

Lemma 1.1.14. Let $X$ be a normed space and $\delta_{X}(\epsilon)$ its modulus of convexity. Then

$$
\begin{aligned}
\delta_{X}(\epsilon) & =\inf \left\{1-\left\|\frac{x+y}{2}\right\|: x, y \in B_{X},\|x-y\| \geq \epsilon\right\} \\
& =\inf \left\{1-\left\|\frac{x+y}{2}\right\|: x, y \in S_{X},\|x-y\| \geq \epsilon\right\} \\
& =\inf \left\{1-\left\|\frac{x+y}{2}\right\|: x, y \in B_{X},\|x-y\|=\epsilon\right\} .
\end{aligned}
$$

Proof. See [Meg12], Theorem 5.2.5.

Proposition 1.1.15. Let $1<p<\infty$ and $X$ be a normed space. $X$ is uniformly convex if and only if, for every $\epsilon>0$ there is a number $\delta_{p}(\epsilon)>0$ such that, for all $x, y \in B_{X}$ such that $\|x-y\|_{X} \geq \epsilon$ we have

$$
\left\|\frac{x+y}{2}\right\|_{X}^{p} \leq \frac{1}{2}\left[\|x\|_{X}^{p}+\|y\|_{X}^{p}\right]\left[1-\delta_{p}(\epsilon)\right]
$$

Therefore, $X$ is uniformly convex if and only if, for all $x, y \in X$, such that $(x, y) \neq 0$,

$$
\left\|\frac{x+y}{2}\right\|_{X}^{p} \leq\left[1-\delta_{p}\left(\frac{\|x-y\|_{X}}{\max \left\{\|x\|_{X},\|y\|_{X}\right\}}\right)\right] \frac{1}{2}\left[\|x\|_{X}^{p}+\|y\|_{X}^{p}\right] .
$$

Proof. See [Bea11], Proposition 1 in Chapter II, Section 1.

Definition 1.1.16. Let $X_{1}, \ldots, X_{n}$ be Banach spaces and $1 \leq p \leq \infty$. We denote by $X_{1} \oplus^{p} \cdots \oplus^{p} X_{n}$ the $p$-direct sum space $X_{1} \times \cdots \times X_{n}$ with the norm given by

$$
\left\|\left(x_{1}, \ldots, x_{n}\right)\right\|_{(p)}=\left(\sum_{j=1}^{n}\left\|x_{j}\right\|_{X_{j}}^{p}\right)^{\frac{1}{p}}, 1 \leq p<\infty
$$




$$
\left\|\left(x_{1}, \ldots, x_{n}\right)\right\|_{(\infty)}=\max _{1 \leq j \leq n}\left\|x_{j}\right\|_{X_{j}}
$$

The space presented in the definition above has the following properties:

(a) $X_{1} \oplus^{p} \cdots \oplus^{p} X_{n}$ is a Banach space.

(b) If $X_{j}, 1 \leq j \leq n$, is reflexive, then $X_{1} \oplus^{p} \cdots \oplus^{p} X_{n}$ is reflexive.

Proposition 1.1.17. If $X_{0}, X_{1}$ are uniformly convex spaces, then for $1<p<\infty, X_{0} \oplus^{p} X_{1}$ is uniformly convex.

Proof. Let $\epsilon>0, x=\left(x_{0}, x_{1}\right), y=\left(y_{0}, y_{1}\right) \in X_{0} \oplus^{p} X_{1}$ be such that $\|x-y\|_{(p)}^{p} \geq \epsilon$. Denote by $\delta_{0}$ and $\delta_{1}$ the moduli of convexity of $X_{0}$ and $X_{1}$, respectively, according to (1.1). Thus,

$$
\begin{aligned}
\left\|\frac{x+y}{2}\right\|_{(p)}^{p} & =\left\|\frac{x_{0}+y_{0}}{2}\right\|_{X_{0}}^{p}+\left\|\frac{x_{1}+y_{1}}{2}\right\|_{X_{1}}^{p} \\
& \leq\left[1-\delta_{0}\left(\frac{\left\|x_{0}-y_{0}\right\|_{X_{0}}}{\max \left\{\left\|x_{0}\right\|_{X_{0}},\left\|y_{0}\right\|_{X_{0}}\right\}}\right)\right] \frac{1}{2}\left[\left\|x_{0}\right\|_{X_{0}}^{p}+\left\|y_{0}\right\|_{X_{0}}^{p}\right] \\
& +\left[1-\delta_{1}\left(\frac{\left\|x_{1}-y_{1}\right\|_{X_{1}}}{\max \left\{\left\|x_{1}\right\|_{X_{1}},\left\|y_{1}\right\|_{X_{1}}\right\}}\right)\right] \frac{1}{2}\left[\left\|x_{1}\right\|_{X_{1}}^{p}+\left\|y_{1}\right\|_{X_{1}}^{p}\right] \\
& =\frac{1}{2}\left[\|x\|_{(p)}^{p}+\|y\|_{(p)}^{p}\right]-\frac{1}{2}\left[\left\|x_{0}\right\|_{X_{0}}^{p}+\left\|y_{0}\right\|_{X_{0}}^{p}\right]\left[\delta_{0}\left(\frac{\left\|x_{0}-y_{0}\right\|_{X_{0}}}{\max \left\{\left\|x_{0}\right\|_{X_{0}},\left\|y_{0}\right\|_{X_{0}}\right\}}\right)\right] \\
& -\frac{1}{2}\left[\left\|x_{1}\right\|_{X_{1}}^{p}+\left\|y_{1}\right\|_{X_{1}}^{p}\right]\left[\delta_{1}\left(\frac{\left\|x_{1}-y_{1}\right\|_{X_{1}}}{\max \left\{\left\|x_{1}\right\|_{X_{1}},\left\|y_{1}\right\|_{X_{1}}\right\}}\right)\right] \\
& \leq \frac{1}{2}\left[\|x\|_{(p)}^{p}+\|y\|_{(p)}^{p}\right]-\frac{1}{2}\left[\max \left\{\left\|x_{0}\right\|_{X_{0}},\left\|y_{0}\right\|_{X_{0}}\right\}\right]^{p}\left[\delta_{0}\left(\frac{\left\|x_{0}-y_{0}\right\|_{X_{0}}}{\max \left\{\left\|x_{0}\right\|_{X_{0}},\left\|y_{0}\right\|_{X_{0}}\right\}}\right)\right] \\
& -\frac{1}{2}\left[\max \left\{\left\|x_{1}\right\|_{X_{1}},\left\|y_{1}\right\|_{X_{1}}\right\}\right]^{p}\left[\delta_{1}\left(\frac{\left\|x_{1}-y_{1}\right\|_{X_{1}}}{\max \left\{\left\|x_{1}\right\|_{X_{1}},\left\|y_{1}\right\|_{X_{1}}\right\}}\right)\right] .
\end{aligned}
$$

Let us now consider some estimates: Suppose, without loss of generality, that

$$
\max \left\{\left\|x_{0}-y_{0}\right\|_{X_{0}}^{p},\left\|x_{1}-y_{1}\right\|_{X_{1}}^{p}\right\}=\left\|x_{0}-y_{0}\right\|_{X_{0}}^{p}
$$

Therefore,

$$
\epsilon \leq\|x-y\|_{(p)}^{p}=\left\|x_{0}-y_{0}\right\|_{X_{0}}^{p}+\left\|x_{1}-y_{1}\right\|_{X_{1}}^{p} \leq 2 \max \left\{\left\|x_{0}-y_{0}\right\|_{X_{0}}^{p},\left\|x_{1}-y_{1}\right\|_{X_{1}}^{p}\right\}=2\left\|x_{0}-y_{0}\right\|_{X_{0}}^{p} .
$$

Moreover, since $\|x\|_{(p)}^{p}=\left\|x_{0}\right\|_{X_{0}}^{p}+\left\|x_{1}\right\|_{X_{1}}^{p} \leq 1$ and $\|y\|_{(p)}^{p}=\left\|y_{0}\right\|_{X_{0}}^{p}+\left\|y_{1}\right\|_{X_{1}}^{p} \leq 1$, it follows that $\max \left\{\left\|x_{0}\right\|_{X_{0}},\left\|y_{0}\right\|_{X_{0}}\right\} \leq 1$. Thus,

$$
\frac{\left\|x_{0}-y_{0}\right\|_{X_{0}}}{\max \left\{\left\|x_{0}\right\|_{X_{0}},\left\|y_{0}\right\|_{X_{0}}\right\}} \geq\left(\frac{\epsilon}{2}\right)^{\frac{1}{p}} .
$$


Since $\delta_{0}$ is non-decreasing, it follows that

$$
\delta_{0}\left(\frac{\left\|x_{0}-y_{0}\right\|_{X_{0}}}{\max \left\{\left\|x_{0}\right\|_{X_{0}},\left\|y_{0}\right\|_{X_{0}}\right\}}\right) \geq \delta_{0}\left(\left(\frac{\epsilon}{2}\right)^{\frac{1}{p}}\right) .
$$

Moreover, since $\left(\frac{\epsilon}{2}\right)^{\frac{1}{p}} \leq\left\|x_{0}-y_{0}\right\|_{X_{0}} \leq 2 \max \left\{\left\|x_{0}\right\|_{X_{0}},\left\|y_{0}\right\|_{X_{0}}\right\}$, we have

$$
\left[\max \left\{\left\|x_{0}\right\|_{X_{0}},\left\|y_{0}\right\|_{X_{0}}\right\}\right]^{p} \delta_{0}\left(\frac{\left\|x_{0}-y_{0}\right\|_{X_{0}}}{\max \left\{\left\|x_{0}\right\|_{X_{0}},\left\|y_{0}\right\|_{X_{0}}\right\}}\right) \geq \frac{\epsilon}{2^{p+1}} \delta_{0}\left(\left(\frac{\epsilon}{2}\right)^{\frac{1}{p}}\right) .
$$

Thus, returning to (1.3), we have

$$
\begin{aligned}
\left\|\frac{x+y}{2}\right\|_{(p)}^{p} & \leq \frac{1}{2}\left[\|x\|_{(p)}^{p}+\|y\|_{(p)}^{p}\right]-\frac{1}{2}\left[\max \left\{\left\|x_{0}\right\|_{X_{0}},\left\|y_{0}\right\|_{X_{0}}\right\}\right]^{p}\left[\delta_{0}\left(\frac{\left\|x_{0}-y_{0}\right\|_{X_{0}}}{\max \left\{\left\|x_{0}\right\|_{X_{0}},\left\|y_{0}\right\|_{X_{0}}\right\}}\right)\right] \\
& -\frac{1}{2}\left[\max \left\{\left\|x_{1}\right\|_{X_{1}},\left\|y_{1}\right\|_{X_{1}}\right\}\right]^{p}\left[\delta_{1}\left(\frac{\left\|x_{1}-y_{1}\right\|_{X_{1}}}{\max \left\{\left\|x_{1}\right\|_{X_{1}},\left\|y_{1}\right\|_{X_{1}}\right\}}\right)\right] \\
& \leq \frac{1}{2}\left[\|x\|_{(p)}^{p}+\|y\|_{(p)}^{p}\right]-\frac{1}{2}\left[\max \left\{\left\|x_{0}\right\|_{X_{0}},\left\|y_{0}\right\|_{X_{0}}\right\}\right]^{p}\left[\delta_{0}\left(\frac{\left\|x_{0}-y_{0}\right\|_{X_{0}}}{\max \left\{\left\|x_{0}\right\|_{X_{0}},\left\|y_{0}\right\|_{X_{0}}\right\}}\right)\right] \\
& \leq \frac{1}{2}\left[\|x\|_{(p)}^{p}+\|y\|_{(p)}^{p}\right]-\frac{\epsilon}{2^{p+2}} \delta_{0}\left(\left(\frac{\epsilon}{2}\right)^{\frac{1}{p}}\right) \leq 1-\frac{\epsilon}{2^{p+2}} \delta_{0}\left(\left(\frac{\epsilon}{2}\right)^{\frac{1}{p}}\right) .
\end{aligned}
$$

Since $X_{0}$ is uniformly convex, it follows that $\frac{\epsilon}{2^{p+2}} \delta_{0}\left(\left(\frac{\epsilon}{2}\right)^{\frac{1}{p}}\right)>0$, hence, $X_{0} \oplus^{p} X_{1}$ is uniformly convex.

Remark 1.1.18. Notice that, from equation (1.4) in the last proof, it follows that the modulus of convexity $\delta$ of $X_{0} \oplus^{p} X_{1}$ is bounded in terms of the modulus of convexity $\delta_{0}$ of $X_{0}$, i.e., we have for every $\epsilon>0, \delta(\epsilon) \geq \frac{\epsilon}{2^{p+2}} \min \left\{\delta_{0}\left(\left(\frac{\epsilon}{2}\right)^{\frac{1}{p}}\right), \delta_{1}\left(\left(\frac{\epsilon}{2}\right)^{\frac{1}{p}}\right)\right\}$.

\section{$1.2 \quad L_{p}$ spaces}

The spaces $L_{p}$ are a very important example in Functional Analysis, and as expected, will often illustrate concepts from the theory developed herein.

In this section we will consider $(\Omega, \mathcal{M}, \mu)$ as a measure space, where $\Omega$ is a set, $\mathcal{M}$ denotes a $\sigma$ algebra and $\mu$ is a positive measure. We will adopt the convention that two functions are equal if they differ only in a set of $\mu$-null measure. The main references used in this section are [Fol99] and [Rud70].

Let us first state some important results in measure theory. We denote $\mathbb{K}=\mathbb{R}$ or $\mathbb{C}$.

Theorem 1.2.1. (Lebesgue's Dominated Convergence Theorem) Suppose that $\left(f_{n}\right)$ is a sequence of complex measurable functions on $\Omega$ such that

$$
f(\omega)=\lim _{n \rightarrow \infty} f_{n}(\omega)
$$


exists for every $\omega \in \Omega$. If there is an integrable function $g$ such that $\left|f_{n}(\omega)\right| \leq|g(\omega)|$ for all $\omega \in \Omega$ and $n \in \mathbb{N}$, then $f$ is integrable,

$$
\lim _{n \rightarrow \infty} \int\left|f_{n}-f\right| d \mu=0
$$

and

$$
\lim _{n \rightarrow \infty} \int f_{n} d \mu=\int f d \mu
$$

Proof. See [Rud70], Theorem 1.34.

Theorem 1.2.2. (Hölder's Inequality) Let $1 \leq p, q<\infty$ be such that $\frac{1}{p}+\frac{1}{q}=1$ (we say that $q$ is the conjugate exponent of $\mathrm{p}$ ), and $f, g: \Omega \longrightarrow \mathbb{K}$ be two measurable functions. Then

$$
\int|f g| d \mu \leq\left(\int|f|^{p} d \mu\right)^{\frac{1}{p}}\left(\int|g|^{q} d \mu\right)^{\frac{1}{q}}
$$

Proof. See [Rud70], Theorem 3.5.

Definition 1.2.3. Consider $1 \leq p \leq \infty$. We denote by $L_{p}(\Omega, \mathcal{M}, \mu)$ (or simply $L_{p}(\Omega, \mu), L_{p}(\Omega)$ or $L_{p}$ ) the Lebesgue space of (all the equivalent classes of) measurable functions $f: \Omega \longrightarrow \mathbb{C}$ such that

$$
\begin{gathered}
\int_{\Omega}|f(\omega)|^{p} d \mu(\omega)<\infty, 1 \leq p<\infty \\
\underset{\omega \in \Omega}{\operatorname{ess} \sup }|f(\omega)|<\infty, p=\infty,
\end{gathered}
$$

equipped with the norm

$$
\begin{gathered}
\|f\|_{L_{p}(\Omega, \mathcal{M}, \mu)}=\left(\int_{\Omega}|f(\omega)|^{p} d \mu(\omega)\right)^{1 / p}, 1 \leq p<\infty \\
\|f\|_{L_{\infty}(\Omega, \mathcal{M}, \mu)}=\underset{\omega \in \Omega}{\operatorname{essipsup}}|f(\omega)|, p=\infty .
\end{gathered}
$$

For $1 \leq p \leq \infty, L_{p}(\Omega, \mathcal{M}, \mu)$ is a Banach space.

Definition 1.2.4. Consider $\mu$ a positive measure on $\mathcal{M}$ and $\lambda$ an arbitraty measure on $\mathcal{M}$. We say that $\lambda$ is absolutely continuous with respect to $\mu$ (and denote by $\lambda \ll \mu$ ) if given $E \in \mathcal{M}$ such that $\mu(E)=0$, we have that $\lambda(E)=0$.

Theorem 1.2.5. (Radon-Nikodým Theorem) Consider $\mu$ and $\lambda$ two $\sigma$-finite measures on the measurable space $(\Omega, \mathcal{M})$, such that $\lambda \ll \mu$. Then there is a positive function $g \in L_{1}(\Omega, \mathcal{M}, \mu)$ such that

$$
\lambda(E)=\int_{E} g d \mu
$$

for all $E \in \mathcal{M}$.

Proof. See [Rud70], Theorem 6.9. 
We consider the following set of simple functions that are zero outside a set of finite measure:

$$
\mathcal{D}=\left\{f=\sum_{n=1}^{m} \alpha_{n} \chi_{E_{n}}: f \text { is a simple function, } m \in \mathbb{N}, \mu\left(E_{n}\right)<\infty, \alpha_{n} \in \mathbb{K}, n=1, \ldots, m\right\} \text {. }
$$

Proposition 1.2.6. If $1 \leq p \leq \infty$, the set $\mathcal{D}$ is dense in $L_{p}$.

Proof. See [Fol99], Proposition 6.7 and Theorem 6.8.

Proposition 1.2.7. If $1 \leq p<q<r \leq \infty$, then $L_{q} \subset L_{p}+L_{r}$.

Proof. We will show that $f \in L_{q}$ is the sum of a function in $L_{p}$ and a function in $L_{r}$. Consider the measurable set $E=\{\omega \in \Omega:|f(\omega)|>1\}$ and the functions $g=f \chi_{E}$ and $h=f \chi_{E^{c}}$. Note that $f=g+h$. Let us show that $g \in L_{p}$ and $h \in L_{r}$. Indeed, for $r \neq \infty$ we have that for all $\omega \in \Omega$

$$
\begin{gathered}
|g(\omega)|^{p}=\left|f(\omega) \chi_{E}(\omega)\right|^{p}=|f(\omega)|^{p} \chi_{E}(\omega) \leq|f(\omega)|^{q} \chi_{E}(\omega) \Rightarrow g \in L_{p} \\
|h(\omega)|^{r}=\left|f(\omega) \chi_{E^{c}}(\omega)\right|^{r}=|f(\omega)|^{r} \chi_{E^{c}}(\omega) \leq|f(\omega)|^{q} \chi_{E^{c}}(\omega) \Rightarrow h \in L_{r}
\end{gathered}
$$

For $r=\infty$ we have that $\sup _{\omega \in \Omega}|h(\omega)|=\sup _{\omega \in \Omega}\left|f(\omega) \chi_{E^{c}}(\omega)\right|=\sup _{\omega \in E^{c}}|f(\omega)| \leq 1$, thus $h \in L_{\infty}$.

Proposition 1.2.8. If $1 \leq p<q<r \leq \infty$, then $L_{p} \cap L_{r} \subset L_{q}$ and $\|f\|_{L_{q}} \leq\|f\|_{L_{p}}^{\theta}\|f\|_{L_{r}}^{1-\theta}$, for every $f \in L_{p} \cap L_{r}$, where $0<\theta<1$ is defined by $\frac{1}{q}=\frac{\theta}{p}+\frac{1-\theta}{r}$.

Proof. Let us first study the case $r \neq \infty$. Consider $f \in L_{p} \cap L_{r}, p^{\prime}=\frac{p}{q \theta}$ and $r^{\prime}=\frac{r}{(1-\theta) q}$; then we have $\frac{1}{p^{\prime}}+\frac{1}{r^{\prime}}=1$ and by Hölder's Inequality (Theorem 1.2.2)

$$
\begin{aligned}
\int|f|^{q} & =\int|f|^{q \theta}|f|^{q(1-\theta)} \leq\left(\int|f|^{q \theta p^{\prime}}\right)^{1 / p^{\prime}}\left(\int|f|^{q(1-\theta) r^{\prime}}\right)^{1 / r^{\prime}} \\
& =\left(\int|f|^{p}\right)^{q \theta / p}\left(\int|f|^{r}\right)^{(1-\theta) q / r}=\|f\|_{L_{p}}^{q \theta}\|f\|_{L_{r}}^{q(1-\theta)}<\infty
\end{aligned}
$$

This shows that $f \in L_{q}$ and $\|f\|_{L_{q}} \leq\|f\|_{L_{p}}^{\theta}\|f\|_{L_{r}}^{1-\theta}$.

For $r=\infty$ we have that $1 / q=\theta / p$ and for all $\omega \in \Omega$ it is valid that $|f(\omega)|^{q}=|f(\omega)|^{q-p}|f(\omega)|^{p} \leq$ $\|f\|_{L_{\infty}}^{q-p}|f(\omega)|^{p}$. Thus, since $p / q=\theta$, we have

$$
\|f\|_{L_{q}}=\left(\int|f|^{q}\right)^{1 / q} \leq\|f\|_{L_{\infty}}^{1-p / q}\left(\int|f|^{p}\right)^{1 / q}=\|f\|_{L_{\infty}}^{1-\theta}\left(\int|f|^{p}\right)^{\theta / p}=\|f\|_{L_{\infty}}^{1-\theta}\|f\|_{L_{p}}^{\theta}<\infty
$$

This shows that $f \in L_{q}$ and $\|f\|_{L_{q}} \leq\|f\|_{L_{p}}^{\theta}\|f\|_{L_{\infty}}^{1-\theta}$.

Theorem 1.2.9. Let $1 \leq p, q<\infty$ with $\frac{1}{q}+\frac{1}{p}=1$ and $f \in L_{p}$, then

$$
\|f\|_{L_{p}}=\sup \left\{\left|\int f g\right|: g \in \mathcal{D},\|g\|_{L_{q}}=1\right\} .
$$


Proof. Note first that if $f=0 \mu$-almost everywhere, the equality is trivial. Suppose then that $f \neq 0$ $\mu$-almost everywhere. Let us start by showing that the equality (1.5) is valid if we consider the supremum over all the functions $g \in L_{q}$ such that $\|g\|_{L_{q}}=1$. Indeed, given such $g \in L_{q}$, we have by Hölder's Inequality (Theorem 1.2.2) that

$$
\left|\int f g\right| \leq \int|f g| \leq\left(\int|f|^{p}\right)^{1 / p}\left(\int|g|^{q}\right)^{1 / q}=\|f\|_{L_{p}}\|g\|_{L_{q}}=\|f\|_{L_{q}},
$$

therefore $\sup \left\{\left|\int f g\right|: g \in L_{q},\|g\|_{L_{q}}=1\right\} \leq\|f\|_{L_{p}}$. In order to prove the other inequality, consider $h: \Omega \longrightarrow \mathbb{C}$ given by

$$
h(\omega)=\frac{e^{-i \arg f(\omega)}|f(\omega)|^{p-1}}{\|f\|_{L_{p}}^{p-1}}, \forall \omega \in \Omega .
$$

Note that, since $q(p-1)=p$,

$$
\|h\|_{L_{q}}^{q}=\int_{\Omega}\left|\frac{e^{-i \arg f(\omega)}|f(\omega)|^{p-1}}{\|f\|_{L_{p}}^{p-1}}\right|^{q} d \mu=\frac{1}{\|f\|_{L_{p}}^{(p-1) q}} \int_{\Omega}|f(\omega)|^{(p-1) q} d \mu=\frac{1}{\|f\|_{L_{p}}^{p}} \int_{\Omega}|f(\omega)|^{p} d \mu=1 .
$$

Thus, $h \in L_{q}$. Moreover,

$$
\begin{aligned}
\int f h & =\int_{\Omega} f(x) \frac{e^{-i \arg f(x)}|f(x)|^{p-1}}{\|f\|_{L_{p}}^{p-1}} d \mu=\int_{\Omega} \frac{|f(x)| e^{i \arg f(x)} e^{-i \arg f(x)}|f(x)|^{p-1}}{\|f\|_{L_{p}}^{p-1}} d \mu \\
& =\frac{1}{\|f\|_{L_{p}}^{p-1}} \int_{\Omega}|f(x)|^{p} d \mu=\|f\|_{L_{p}}^{1-p}\|f\|_{L_{p}}^{p}=\|f\|_{L_{p}},
\end{aligned}
$$

from which follows that $\sup \left\{\left|\int f g\right|: g \in L_{q},\|g\|_{L_{q}}=1\right\} \geq\|f\|_{L_{p}}$, and therefore

$$
\sup \left\{\left|\int f g\right|: g \in L_{q},\|g\|_{L_{q}}=1\right\}=\|f\|_{L_{p}} \text {. }
$$

Now let us show that

$$
\sup \left\{\left|\int f g\right|: g \in \mathcal{D},\|g\|_{L_{q}}=1\right\}=\sup \left\{\left|\int f g\right|: g \in L_{q},\|g\|_{L_{q}}=1\right\} .
$$

It is immediate that

$$
\sup \left\{\left|\int f g\right|: g \in \mathcal{D},\|g\|_{L_{q}}=1\right\} \leq \sup \left\{\left|\int f g\right|: g \in L_{q},\|g\|_{L_{q}}=1\right\} .
$$

For the other inequality, consider $\epsilon>0$. By the supremum property, there is $g_{0} \in L_{q}$, with $\left\|g_{0}\right\|_{L_{q}}=1$ such that

$$
\sup \left\{\left|\int f g\right|: g \in L_{q},\|g\|_{L_{q}}=1\right\} \leq\left|\int f g_{0}\right|+\frac{\epsilon}{2}
$$

Moreover, by Proposition 1.2.6, there is a simple function $h_{0} \in \mathcal{D}$ such that

$$
\left\|g_{0}-h_{0}\right\|_{L_{q}}<\frac{\epsilon}{2\|f\|_{L_{p}}} .
$$


From Hölder's Inequality (Theorem 1.2.2) we have

$$
\begin{aligned}
\sup \left\{\left|\int f g\right|: g \in L_{q},\|g\|_{L_{q}}=1\right\} & \leq\left|\int f g_{0}\right|+\frac{\epsilon}{2}=\left|\int f\left(h_{0}+g_{0}-h_{0}\right)\right|+\frac{\epsilon}{2} \\
& \leq\left|\int f\left(g_{0}-h_{0}\right)\right|+\left|\int f h_{0}\right|+\frac{\epsilon}{2} \\
& \leq\left|\int f h_{0}\right|+\int\left|f \| g_{0}-h_{0}\right|+\frac{\epsilon}{2} \\
& \leq\left|\int f h_{0}\right|+\|f\|_{L_{p}}\left\|g_{0}-h_{0}\right\|_{L_{q}}+\frac{\epsilon}{2} \\
& \leq \sup \left\{\left|\int f g\right|: g \in \mathcal{D},\|g\|_{L_{q}}=1\right\}+\|f\|_{L_{p}}\left\|g_{0}-h_{0}\right\|_{L_{q}}+\frac{\epsilon}{2} \\
& <\sup \left\{\left|\int f g\right|: g \in \mathcal{D},\|g\|_{L_{q}}=1\right\}+\|f\|_{L_{p}} \frac{\epsilon}{2\|f\|_{L_{p}}}+\frac{\epsilon}{2} \\
& =\sup \left\{\left|\int f g\right|: g \in \mathcal{D},\|g\|_{L_{q}}=1\right\}+\epsilon .
\end{aligned}
$$

Since $\epsilon>0$ is arbitrary, it follows that

$$
\sup \left\{\left|\int f g\right|: g \in \Sigma,\|g\|_{L_{q}}=1\right\}=\sup \left\{\left|\int f g\right|: g \in L_{q},\|g\|_{L_{q}}=1\right\}=\|f\|_{L_{p}} .
$$

Theorem 1.2.10. If $1<p<\infty$, then $L_{p}(\Omega, \mathcal{M}, \mu)$ is uniformly convex.

Proof. See [Meg12], Theorem 5.2.11.

The result also follows from Proposition 1.5.13 for $X=\mathbb{C}$.

Note that for $1<p<\infty, L_{p}(\Omega, \mathcal{M}, \mu)$ is also strictly convex.

The spaces defined in the following definition will also often serve as examples in this text.

Definition 1.2.11. Let $1 \leq p \leq \infty,(\Omega, \mathcal{M}, \mu)$ be a positive measure space and $w: \Omega \longrightarrow[0, \infty)$ be a measurable function. We define the space $L_{p}(w)$, the $w$-weighted $L_{p}$ space, as the space of all equivalent classes of measurable functions $f: \Omega \longrightarrow \mathbb{C}$ such that

$$
\begin{gathered}
\int_{\Omega}|f(x)|^{p} w(x) d \mu(x)<\infty, 1 \leq p<\infty \\
\underset{x \in \Omega}{\operatorname{ess} \sup }|f(x) w(x)|<\infty, p=\infty,
\end{gathered}
$$

equipped with the norm

$$
\begin{gathered}
\|f\|_{L_{p}(w)}=\left(\int_{\Omega}|f(x)|^{p} w(x) d \mu(x)\right)^{1 / p}, 1 \leq p<\infty \\
\|f\|_{L_{\infty}(w)}=\underset{x \in \Omega}{\operatorname{ess} \sup }|f(x) w(x)|, p=\infty .
\end{gathered}
$$

Note that if we consider the measure $\nu=w d \mu$, then $L_{p}(w)=L_{p}(\Omega, \mathcal{M}, \nu)$. Thus, for $1<p<\infty$ we 
have that $L_{p}(w)$ is uniformly convex.

\subsection{Hardy Spaces}

In this section we introduce some concepts of the theory of Hardy Spaces. Hardy Spaces were introduced by F. Riesz in [Rie23] after the work of G. H. Hardy in [Har15].

We denote by $\mathbb{D}$ the unit open disk centered at the origin in $\mathbb{C}, \mathbb{T}=\partial \mathbb{D}$ the boundary of the disk and $m$ the normalized Lesbesgue measure over $\mathbb{T}$. The main references used in this section are [Pis16], [Dur70] and [Koo98].

Definition 1.3.1. Let $1 \leq p<\infty$. The Hardy Space $H^{p}(\mathbb{D})$ is defined as the space of all holomorphic functions $f: \mathbb{D} \longrightarrow \mathbb{C}$ such that

$$
\sup _{0 \leq r<1} \frac{1}{2 \pi} \int_{0}^{2 \pi}\left|f\left(r e^{i t}\right)\right|^{p} d t<\infty
$$

equipped with the norm

$$
\|f\|_{H^{p}(\mathbb{D})}=\sup _{0 \leq r<1}\left(\frac{1}{2 \pi} \int_{0}^{2 \pi}\left|f\left(r e^{i t}\right)\right|^{p} d t\right)^{\frac{1}{p}}
$$

The space $H^{\infty}(\mathbb{D})$ is the space of bounded holomorphic functions $f$ on $\mathbb{D}$ equipped with the supremum norm:

$$
\|f\|_{H^{\infty}(\mathbb{D})}=\sup _{z \in \mathbb{D}}|f(z)| .
$$

Note that if $1 \leq p<q \leq \infty$, we have $H^{q}(\mathbb{D}) \subseteq H^{p}(\mathbb{D})$.

For all $r \in(0,1)$ and for all $\theta \in \mathbb{T}$ put

$$
P_{r}(\theta)=\frac{1-r^{2}}{r^{2}-2 r \cos \theta+1}
$$

If $z=r e^{i \theta} \in \mathbb{D}$ put $P_{z}(t)=P_{r}(\theta-t) . P_{r}$ is called the Poisson kernel for the unit disk.

Recall the following result:

Theorem 1.3.2. (Fatou's Theorem) Consider $1 \leq p \leq \infty$. For every $f \in H^{p}(\mathbb{D})$ the radial limit

$$
f^{*}\left(e^{i t}\right)=\lim _{r \rightarrow 1^{-}} f\left(r e^{i t}\right)
$$

exists almost everywhere in $t$. We also have that $f^{*} \in L_{p}(\mathbb{T})$ and $\|f\|_{H^{p}(\mathbb{D})}=\left\|f^{*}\right\|_{L_{p}(\mathbb{T})}$.

Proof. See [Rud70], Theorem 11.20.

Moreover, $f$ satisfies the Poisson Integral for each $z=r e^{i \theta} \in \mathbb{D}$, that is,

$$
f(z)=\int_{\mathbb{T}} f^{*}\left(e^{i t}\right) P_{r}(\theta-t) d m(t) .
$$


Thus, we can identify $f$ with $f^{*}$ and regard $H^{p}(\mathbb{D})$ as a closed subspace of $L_{p}(\mathbb{T})$.

Theorem 1.3.3. A holomorphic function $f: \mathbb{D} \longrightarrow \mathbb{C}$ is representable in the form

$$
f(z)=\int_{\mathbb{T}} \varphi\left(e^{i t}\right) P_{r}(\theta-t) d m(t)
$$

as the Poisson integral of a function $\varphi \in L_{p}(\mathbb{T})(1 \leq p \leq \infty)$ if and only if $f \in H^{p}(\mathbb{D})$. In this case, $\varphi\left(e^{i t}\right)=f^{*}\left(e^{i t}\right)$ a.e.

Proof. See [Dur70], Theorem 3.1.

In this text, to facilitate notation, we will identify $f \in H^{p}(\mathbb{D})$ with $f \in L_{p}(\mathbb{T})$ omitting the $*$ in the radial limit.

Theorem 1.3.4. For $1 \leq p \leq \infty$, if $f \in H^{p}(\mathbb{D})$ and $z=r e^{i \theta} \in \mathbb{D}$, we have

$$
\log |f(z)| \leq \int_{\mathbb{T}} \log \left|f\left(e^{i t}\right)\right| P_{r}(\theta-t) d m(t) \leq \frac{1+r}{1-r} \int_{\mathbb{T}} \log \left|f\left(e^{i t}\right)\right| d m(t) .
$$

Proof. See [Dur70], Theorem 2.7 .

Definition 1.3.5. Let $1 \leq p \leq \infty$. An outer function for the space $H^{p}(\mathbb{D})$ is a function of the form

$$
F(z)=e^{i \alpha} \exp \left(\int_{\mathbb{T}} \frac{e^{i t}+z}{e^{i t}-z} \log \psi\left(e^{i t}\right) d m(t)\right), z \in \mathbb{D}
$$

where $\alpha \in \mathbb{R}, \psi \in L_{p}(\mathbb{T}), \log \psi \in L_{1}(\mathbb{T})$ and $\psi \geq 0$.

Definition 1.3.6. A function of the form

$$
B(z)=z^{m} \prod_{n} \frac{\left|a_{n}\right|}{a_{n}} \frac{a_{n}-z}{1-\overline{a_{n}} z}, z \in \mathbb{D}
$$

is called a Blaschke product, where $m \in \mathbb{N}, \sum\left(1-\left|a_{n}\right|\right)<\infty$ and $\left\{a_{n}\right\}$ is a set of complex numbers that may be finite, or even empty. If $\left\{a_{n}\right\}=\varnothing$, we consider $B(z)=z^{m}$.

Definition 1.3.7. A function of the form

$$
S(z)=\exp \left(-\int_{\mathbb{T}} \frac{e^{i t}+z}{e^{i t}-z} d \mu(t)\right), z \in \mathbb{D}
$$

is called a singular inner function, where $\mu: \mathbb{T} \longrightarrow \mathbb{R}$ is a bounded nondecreasing function such that $\mu^{\prime}(t)=0$ a.e.

The Hardy spaces satisfy the following characterization: 
Theorem 1.3.8. (Canonical Factorization Theorem) Let $1 \leq p \leq \infty$. Every function $f \in H^{p}(\mathbb{D})$, $f \equiv 0$, has a unique factorization of the form $f=B S F$, where $B$ is a Blaschke product, $S$ is a singular inner function, and $F$ is an outer function for the class $H^{p}(\mathbb{D})$, with

$$
F(z)=e^{i \alpha} \exp \left(\int_{\mathbb{T}} \frac{e^{i t}+z}{e^{i t}-z} \log \left|f\left(e^{i t}\right)\right| d m(t)\right), z \in \mathbb{D}
$$

for some $\alpha \in \mathbb{R}$. Conversely, every such product $B S F$ belongs to $H^{p}(\mathbb{D})$.

Proof. See [Dur70], Theorem 2.8 .

Theorem 1.3.9. Given a positive function $\varphi \in L_{p}(\mathbb{T})$, there exist $f \in H^{p}(\mathbb{D})$ such that $\varphi\left(e^{i t}\right)=$ $\left|f\left(e^{i t}\right)\right|$ for almost every $t \in \mathbb{T}$, if and only if

$$
\int_{\mathbb{T}} \log \varphi\left(e^{i t}\right) d m(t)>-\infty
$$

Proof. See [Pis16], Theorem 4.12.

Corollary 1.3.10. Given $\varphi$ as in Theorem 1.3.9, there is a unique outer function $G \in H^{p}(\mathbb{D})$ such that $\left|G\left(e^{i t}\right)\right|=\varphi\left(e^{i t}\right)$ for almost every $t \in \mathbb{T}$, and $G(0)>0$.

Proof. See [Pis16], Corollary 4.13.

\subsection{Holomorphic Functions in Banach Spaces}

We now want to define holomorphic vector-valued functions. A natural way to do that is by analogy with the scalar valued case, defining the derivative of $f$ through limits. Since we are considering functions taking values in a Banach space another natural way of defining holomorphic function is trough a "weak property". We will see that these two possible definitions are equivalent and the latter will allow us to extend classical results from the theory of complex functions to the case of Banach space valued functions. The main references used in this section are [Ahl66], [Rem98], [RS12] and [Lim87].

In the following, $\Omega$ is a nonempty, open and connected subset of $\mathbb{C}, X$ is a complex Banach space and $\mathbb{S}=\{z \in \mathbb{C}: 0<\mathfrak{R} z<1\}$ is the strip in the complex plane.

Let us first state some results of the classical theory of holomorphic functions.

Theorem 1.4.1. (Cauchy's Integral Formula) Let $f: \Omega \longrightarrow \mathbb{C}$ be a holomorphic function and let $D$ be an open disk such that $\bar{D} \subset \Omega$. Then for all $\omega \in D$

$$
f(\omega)=\frac{1}{2 \pi i} \int_{\partial D} \frac{f(z)}{z-w} d z .
$$

and

$$
f^{(k)}(w)=\frac{k !}{2 \pi i} \int_{\partial D} \frac{f(z)}{(z-w)^{k+1}} d z, \forall k \in \mathbb{N} .
$$


Proof. See [Rem98].

Theorem 1.4.2. (Cauchy's Integral Theorem) Let $f: \Omega \longrightarrow \mathbb{C}$ be a holomorphic function. Then $\int_{\gamma} f(z) d z=0$ for every closed piecewise $C^{1}$ curve in $\Omega$.

Proof. See [Rem98].

The converse of the previous theorem is also true.

Theorem 1.4.3. (Morera's Theorem) Let $f: \Omega \longrightarrow \mathbb{C}$ be a continuous function. If

$$
\int_{\gamma} f(z) d z=0
$$

for every closed piecewise $C^{1}$ curve $\gamma$ in $\Omega$, then $f$ is holomorphic on $\Omega$.

Proof. See [Ahl66].

Theorem 1.4.4. (Maximum Modulus Principle) Suppose that $\Omega$ is a bounded region of the complex plane and $f: \bar{\Omega} \longrightarrow \mathbb{C}$ is a continuous function which is holomorphic on $\Omega$. Then the maximum of the function $|f|$ over $\bar{\Omega}$ is assumed on the boundary $\partial \Omega$ of $\Omega$ :

$$
|f(z)| \leq|f|_{\partial \Omega}, \quad \forall z \in \bar{\Omega} .
$$

Proof. See [Rem98].

Remark 1.4.5. Note that the Maximum Modulus Principle implies the following: under the same assumptions, if there is $z_{0} \in \Omega$ such that $\left|f\left(z_{0}\right)\right| \geq|f(z)|$ for all $z \in \Omega$, then $f$ is constant on $\bar{\Omega}$.

Lemma 1.4.6. (Phragmén-Lindelöf Maximum Principle) Let $f: \overline{\mathbb{S}} \longrightarrow \mathbb{C}$ be a continuous and bounded function which is holomorphic on $\mathbb{S}$. If $|f(i t)| \leq M$ and $|f(1+i t)| \leq M$, for all $t \in \mathbb{R}$, then $|f(z)| \leq M, \forall z \in \overline{\mathbb{S}}$.

Proof. Suppose first that for all $\theta \in(0,1), f(\theta+i t) \longrightarrow 0$ when $t \longrightarrow \pm \infty$. Then there is $t_{0} \in \mathbb{R}$ such that $|f(\theta+i t)| \leq M, \forall|t|>\left|t_{0}\right|$ and for all $0<\theta<1$. Applying the Maximum Modulus Principle to the rectangle $R=\left\{z \in \mathbb{C}:|\mathfrak{I} z| \leq t_{0}, 0 \leq \mathfrak{R} z \leq 1\right\}$ we have that $|f(z)| \leq M, \forall \in R$.

For the general case, for each $n \in \mathbb{N}$ consider the function

$$
f_{n}(z)=f(z) e^{z^{2} / n}=f(\theta+i t) e^{\left(\theta^{2}-t^{2}\right) / n} e^{2 i \theta t / n} .
$$


Note that for all $\theta \in(0,1)$ and for all $n \in \mathbb{N}, f_{n}(\theta+i t) \longrightarrow 0$ when $t \longrightarrow \pm \infty$. Moreover,

$$
\begin{gathered}
\left|f_{n}(i t)\right|=|f(i t)| e^{-t^{2} / n} \leq M \\
\left|f_{n}(1+i t)\right|=|f(1+i t)| e^{\left(1-t^{2}\right) / n}=|f(1+i t)| e^{1 / n} e^{-t^{2} / n} \leq e^{1 / n} M
\end{gathered}
$$

Thus, by the previous case, we have that $\left|f_{n}(z)\right| \leq M e^{1 / n}, \forall z \in \overline{\mathbb{S}}, \forall n \in \mathbb{N}$. Consequently,

$$
\left|f_{n}(z)\right|=|f(z)| e^{\left(\theta^{2}-t^{2}\right) / n} \leq M e^{1 / n} \Rightarrow|f(z)| \leq M e^{1 / n} e^{-\left(\theta^{2}-t^{2}\right) / n} .
$$

Fixing $z \in \overline{\mathbb{S}}$ and taking $n \rightarrow \infty$ in (1.7) we have that $|f(z)| \leq M, \forall z \in \overline{\mathbb{S}}$.

We will now present the theory of holomorphic functions taking values in Banach spaces.

Definition 1.4.7. Let $f: \Omega \longrightarrow X$. We say that

(a) $f$ is strongly holomorphic on $\Omega$ if for all $z_{0} \in \Omega$ the limit

$$
\lim _{z \rightarrow z_{0}} \frac{f(z)-f\left(z_{0}\right)}{z-z_{0}}
$$

exists. In this case, we denote the limit by $f^{\prime}\left(z_{0}\right)$.

(b) $f$ is weakly holomorphic on $\Omega$ if for all $z_{0} \in \Omega$ the limit

$$
\lim _{z \rightarrow z_{0}} \frac{\varphi(f(z))-\varphi\left(f\left(z_{0}\right)\right)}{z-z_{0}}
$$

exists for all $\varphi \in X^{*}$.

In other words, $f$ is weakly holomorphic on $\Omega$ if for all $\varphi \in X^{*}$, the function $F: \Omega \longrightarrow \mathbb{C}$ defined by $F(z)=\varphi(f(z))$ is holomorphic on $\Omega$ in the usual (scalar) sense.

Theorem 1.4.8. $f: \Omega \longrightarrow \mathbb{C}$ is strongly holomorphic on $\Omega$ if and only if $f$ is weakly holomorphic on $\Omega$.

Proof. $(\Rightarrow)$ Let $\varphi \in X^{*}$ and $z_{0} \in \Omega$. Then, by the continuity of $\varphi$ we have

$$
\left|\frac{\varphi(f(z))-\varphi\left(f\left(z_{0}\right)\right)}{z-z_{0}}-\varphi\left(f^{\prime}\left(z_{0}\right)\right)\right|=\left|\varphi\left(\frac{f(z)-f\left(z_{0}\right)}{z-z_{0}}-f^{\prime}\left(z_{0}\right)\right)\right| \leq\|\varphi\|_{X^{*}}\left\|\frac{f(z)-f\left(z_{0}\right)}{z-z_{0}}-f^{\prime}\left(z_{0}\right)\right\|_{X}
$$

Therefore, assuming that $f^{\prime}\left(z_{0}\right)=\lim _{z \rightarrow z_{0}} \frac{f(z)-f\left(z_{0}\right)}{z-z_{0}}$, we have that $\varphi\left(f^{\prime}\left(z_{0}\right)\right)=\lim _{z \rightarrow z_{0}} \frac{\varphi(f(z))-\varphi\left(f\left(z_{0}\right)\right)}{z-z_{0}}$. $(\Leftarrow)$ Suppose now that $f$ is weakly holomorphic. Let $D$ be an open disk such that $z_{0} \in D$ and together with its boundary $\partial D$ lies wholly in $\Omega$. For each $\varphi \in X^{*}$ we have that the function $F_{\varphi}(z)=\varphi(f(z))$ is holomorphic on $\Omega$. By the Cauchy Integral Formula (Theorem 1.4.1) applied to $F_{\varphi}$ and $F_{\varphi}^{\prime}$ we 
have

$$
\begin{aligned}
\frac{\varphi(f(z))-\varphi\left(f\left(z_{0}\right)\right)}{z-z_{0}}-\frac{d}{d z}\left(\varphi\left(f\left(z_{0}\right)\right)\right) & =\frac{F_{\varphi}(z)-F_{\varphi}\left(z_{0}\right)}{z-z_{0}}-F_{\varphi}^{\prime}\left(z_{0}\right) \\
& =\frac{1}{2 \pi i} \int_{\partial D}\left[\left(\frac{F_{\varphi}(\omega)}{\omega-z}-\frac{F_{\varphi}(\omega)}{\omega-z_{0}}\right) \frac{1}{z-z_{0}}-\frac{F_{\varphi}(\omega)}{\left(\omega-z_{0}\right)^{2}}\right] d \omega \\
& =\frac{1}{2 \pi i} \int_{\partial D}\left[\frac{1}{z-z_{0}}\left(\frac{1}{\omega-z}-\frac{1}{\omega-z_{0}}\right)-\frac{1}{\left(\omega-z_{0}\right)^{2}}\right] \varphi(f(\omega)) d \omega .
\end{aligned}
$$

For each $\omega \in \partial D$, consider the operator

$$
\begin{aligned}
\Psi_{\omega}: X^{*} & \longrightarrow \mathbb{C} \\
x^{*} & \longmapsto x^{*}(f(\omega))
\end{aligned}
$$

Note that $\left|\Psi_{\omega}\left(x^{*}\right)\right|=\left|x^{*}(f(\omega))\right| \leq\left\|x^{*}\right\|_{X^{*}}\|f(\omega)\|_{X}$, thus $\Psi_{\omega} \in X^{* *}$. Also, since $\partial D$ is compact and $F_{x^{*}}$ is continuous on $\partial D$, there is $C_{x^{*}}>0$ such that $\left|F_{x^{*}}(\omega)\right|=\left|x^{*}(f(\omega))\right| \leq C_{x^{*}}, \forall \omega \in \partial D$.

Consider the family $\mathcal{F}=\left\{\Psi_{\omega}: \omega \in \partial D\right\}$. For all $x^{*} \in X^{*}$ we have $\sup \left\{\left|\Psi_{\omega}\left(x^{*}\right)\right|: \Psi_{\omega} \in \mathcal{F}\right\}=$ $\sup \{|\gamma(f(\omega))|: \omega \in \partial D\} \leq C_{x^{*}}$.

By the Uniform Boundness Principle, we have that $\sup \left\{\left\|\Psi_{\omega}\right\|_{X^{* *}}: \omega \in \partial D\right\} \leq C<\infty$, for some $C>0$. Thus

$$
\sup \left\{\left\|\Psi_{\omega}\right\|_{X^{* *}}: \omega \in \partial D\right\}=\sup \left\{\sup _{x^{*} \in X^{*}} \frac{\left|\Psi_{\omega}\left(x^{*}\right)\right|}{\left\|x^{*}\right\|_{X^{*}}}: \omega \in \partial D\right\}=\sup \left\{\sup _{x^{*} \in X^{*}} \frac{\left|x^{*}(f(\omega))\right|}{\left\|x^{*}\right\|_{X^{*}}}: \omega \in \partial D\right\} \leq C
$$

Therefore, $\sup _{x^{*} \in X^{*}} \frac{\left|x^{*}(f(\omega))\right|}{\left\|x^{*}\right\|_{X^{*}}} \leq C$ for all $\omega \in \partial D$, which implies that $|\varphi(f(\omega))| \leq\|\varphi\|_{X^{*}} C$ for all $\omega \in \partial D$. Therefore, from (1.8) we have

$$
\left|\frac{\varphi(f(z))-\varphi\left(f\left(z_{0}\right)\right)}{z-z_{0}}-\frac{d}{d z}\left(\varphi\left(f\left(z_{0}\right)\right)\right)\right| \leq \frac{1}{2 \pi} \int_{\partial D}\left|\frac{1}{z-z_{0}}\left(\frac{1}{\omega-z}-\frac{1}{\omega-z_{0}}\right)-\frac{1}{\left(\omega-z_{0}\right)^{2}}\right| d \omega\|\varphi\|_{X^{*}} C
$$

that goes to zero as $z \rightarrow z_{0}$.

Remark 1.4.9. If a function $f: \Omega \longrightarrow X$ satisfies one (and therefore both) conditions of Definition 1.4.7, we simply say that $f$ is holomorphic.

As expected, Banach-valued holomorphic functions are continuous:

Proposition 1.4.10. If $f: \Omega \longrightarrow X$ is holomorphic, then it is continuous.

Proof. We have, for $z \neq z_{0} \in \Omega$

$$
\left\|f(z)-f\left(z_{0}\right)\right\|_{X}=\left\|\frac{f(z)-f\left(z_{0}\right)}{z-z_{0}}\left(z-z_{0}\right)\right\|_{X}=\left|z-z_{0}\right|\left\|\frac{f(z)-f\left(z_{0}\right)}{z-z_{0}}\right\|_{X} \stackrel{z \rightarrow z_{0}}{\longrightarrow} 0
$$


Theorem 1.4.11. (Phragmen-Lindelöff Maximum Principle for Banach spaces) Let $f$ : $\overline{\mathbb{S}} \longrightarrow X$ be continuous and bounded, and holomorphic on $\mathbb{S}$. Then

$$
\sup _{z \in \overline{\mathbb{S}}}\|f(z)\|_{X} \leq \max \left\{\sup _{t \in \mathbb{R}}\|f(i t)\|_{X}, \sup _{t \in \mathbb{R}}\|f(1+i t)\|_{X}\right\} .
$$

Proof. Theorem 1.4.6 shows us that the result is valid for $X=\mathbb{C}$. Given $z_{0} \in \mathbb{S}$, we have that $f\left(z_{0}\right) \in X$. It follows from the Hahn-Banach Theorem (Corollary 1.1.4) that there is $\varphi \in X^{*}$ such that $\varphi\left(f\left(z_{0}\right)\right)=\left\|f\left(z_{0}\right)\right\|_{X}$ and $\|\varphi\|_{X^{*}}=1$. Also, $F(z) \doteq \varphi(f(z))$ is holomorphic at $z_{0}$ (in the usual sense); then

$$
|F(z)| \leq \max \left\{\sup _{t \in \mathbb{R}}|F(i t)|, \sup _{t \in \mathbb{R}}|F(1+i t)|\right\}
$$

Now, for all $t \in \mathbb{R}$ we have

$$
\begin{gathered}
|F(i t)|=|\varphi(f(i t))| \leq\|\varphi\|_{X^{*}}\|f(i t)\|_{X}=\|f(i t)\|_{X} \\
|F(1+i t)|=|\varphi(f(1+i t))| \leq\|\varphi\|_{X^{*}}\|f(1+i t)\|_{X}=\|f(1+i t)\|_{X}
\end{gathered}
$$

Replacing in (1.9), we have

$$
\left|\varphi\left(f\left(z_{0}\right)\right)\right|=\left\|f\left(z_{0}\right)\right\|_{X} \leq \max \left\{\sup _{t \in \mathbb{R}}\|f(i t)\|_{X}, \sup _{t \in \mathbb{R}}\|f(1+i t)\|_{X}\right\} .
$$

Since the chosen $z_{0}$ is arbitrary, the result follows.

\subsection{Bochner Integral}

The Bochner Integral extends the definition of Lebesgue Integral to functions taking values in Banach spaces. In this Section we will present definitions and results in this direction. We will see that many of the results of classical Lebesgue theory can be extended to this context. The main references used in this section are [DUJ43] and [Mik78].

Let $(\Omega, \mathcal{M}, \mu)$ be a measure space and $X$ a Banach space.

Definition 1.5.1. A function $f: \Omega \longrightarrow X$ is called simple if there are $x_{1}, \ldots, x_{n} \in X$ and $E_{1}, \ldots, E_{n} \in \mathcal{M}$ such that

$$
f=\sum_{i=1}^{n} x_{i} \chi_{E_{i}}
$$

We define the Bochner Integral of a simple function in a measurable set $E \in \Sigma$ as

$$
\int_{E} f d \mu=\sum_{i=1}^{n} \mu\left(E \cap E_{i}\right) x_{i}
$$

Definition 1.5.2. A function $f: \Omega \longrightarrow X$ is called Bochner measurable if there is a sequence of simple functions $\left(f_{n}\right)$ such that $\lim _{n \rightarrow \infty}\left\|f_{n}-f\right\|_{X}=0 \mu$-almost everywhere. 
Definition 1.5.3. A Bochner measurable function $f: \Omega \longrightarrow X$ is called Bochner integrable if there is a sequence $\left(f_{n}\right)$ of simple functions such that

$$
\lim _{n} \int_{\Omega}\left\|f_{n}-f\right\|_{X} d \mu=0
$$

In this case, the integral of $f$ in a measurable set $E \in \mathcal{M}$ is defined by

$$
\int_{E} f d \mu=\lim _{n} \int_{E} f_{n} d \mu \text {. }
$$

It is possible to show that the Bochner integral of a function is well-defined, i.e. it does not depend on the choice of the sequence $\left(f_{n}\right)$.

Lemma 1.5.4. If $f$ is a Bochner integrable function, then $\varphi f$ is Bochner integrable for all $\varphi \in X^{*}$.

Proof. Let us see first that $\varphi f$ is measurable: Indeed, if $\left(f_{n}\right)$ is a sequence of simple functions such that $\lim _{n}\left\|f_{n}-f\right\|_{X}=0$, then $\left(\varphi f_{n}\right)$ is a sequence of simple functions and we have $\left|\varphi f_{n}-\varphi f\right| \leq$ $\|\varphi\|_{X^{*}}\left\|f_{n}-f\right\|_{X} \rightarrow 0$ as $n \rightarrow \infty$. Moreover,

$$
\lim _{n} \int\left|\varphi f_{n}-\varphi f\right| \leq \int\|\varphi\|_{X^{*}}\left\|f_{n}-f\right\|_{X}=\|\varphi\|_{X^{*}} \int\left\|f_{n}-f\right\|_{X} \rightarrow 0 \text { as } n \rightarrow \infty .
$$

Theorem 1.5.5. (Hille's Theorem) If $f$ is a Bochner integrable function, then for all $\varphi \in X^{*}$ and for all $E \in \mathcal{M}$ we have

$$
\varphi\left(\int_{E} f \mu\right)=\int_{E} \varphi f d \mu .
$$

Proof. Let us show first that (1.10) is valid for simple functions. Let $f=\sum_{i=1}^{n} \chi_{E_{i}} x_{i}$, with $E_{i} \cap E_{j}=\varnothing$, if $i \neq j$. We have

$$
\varphi\left(\int_{E} f d \mu\right)=\varphi\left(\sum_{i=1}^{n} x_{i} \mu\left(E_{i}\right)\right)=\sum_{i=1}^{n} \mu\left(E_{i}\right) \varphi\left(x_{i}\right)
$$

and

$$
\int_{E} \varphi f d \mu=\int_{E} \varphi\left(\sum_{i=1}^{n} x_{i} \chi_{E_{i}}\right) d \mu=\int_{E} \sum_{i=1}^{n} \chi_{E_{i}} \varphi\left(x_{i}\right) d \mu=\sum_{i=1}^{n} \mu\left(E_{i}\right) \varphi\left(x_{i}\right),
$$

which proves the equality in the case of simple functions. Now, let $f$ be any Bochner integrable function and $\left(f_{n}\right)$ a sequence of simple functions such that $\int_{E} f d \mu=\lim _{n} \int_{E} f_{n} d \mu$. Note first that, since $\varphi$ is continuous, we have

$$
\varphi\left(\int_{E} f \mu\right)=\varphi\left(\lim _{n} \int_{E} f_{n} d \mu\right)=\lim _{n} \varphi\left(\int_{E} f_{n} d \mu\right),
$$

then, since the result is valid for simple functions and using properties of the Lebesgue integral and 
the fact that $f$ is Bochner integrable, we have

$$
\begin{aligned}
\left|\varphi\left(\int_{E} f_{n} d \mu\right)-\int_{E} \varphi f d \mu\right| & =\left|\int_{E} \varphi f_{n}-\int \varphi f d \mu\right| \leq \int_{E}\left|\varphi\left(f_{n}-f\right)\right| d \mu \\
& \leq \int_{E}\|\varphi\|_{X^{*}}\left\|f_{n}-f\right\|_{X}=\|\varphi\|_{X^{*}} \int_{E}\left\|f_{n}-f\right\|_{X} \rightarrow 0 \text { as } n \rightarrow \infty
\end{aligned}
$$

Therefore,

$$
\varphi\left(\int_{E} f d \mu\right)=\lim _{n} \varphi\left(\int_{E} f_{n} d \mu\right)=\int_{E} \varphi f d \mu
$$

Let us see that the Cauchy Integral Formula is also valid in the case of vector valued functions.

Theorem 1.5.6. Let $\Omega$ be a nonempty, open and connected subset of $\mathbb{C}, f: \Omega \longrightarrow X$ a holomorphic function and $D$ an open disk such that $\bar{D} \subset \Omega$. Then for all $z \in D$ we have

$$
f(z)=\frac{1}{2 \pi i} \int_{\partial D} \frac{f(\omega)}{\omega-z} d \mu(\omega) .
$$

Proof. For all $\varphi \in X^{*}$, using the Cauchy Integral Formula (Theorem 1.4.1) and Hille's Theorem (Theorem 1.5.5), we have that

$$
\begin{aligned}
\varphi(f(z)) & =\frac{1}{2 \pi i} \int_{\partial D} \frac{\varphi(f(\omega))}{\omega-z} d \mu(\omega)=\frac{1}{2 \pi i} \int_{\partial D} \varphi\left(\frac{f(\omega)}{\omega-z}\right) d \mu(\omega) \\
& =\frac{1}{2 \pi i} \varphi\left(\int_{\partial D} \frac{f(\omega)}{\omega-z} d \mu(\omega)\right)=\varphi\left(\frac{1}{2 \pi i} \int_{\partial D} \frac{f(\omega)}{\omega-z} d \mu(\omega)\right) .
\end{aligned}
$$

By the Hanh-Banach Theorem (separation of points) it follows that

$$
f(z)=\frac{1}{2 \pi i} \int_{\partial D} \frac{f(\omega)}{\omega-z} d \mu(\omega) .
$$

Proposition 1.5.7. Let $u: \overline{\mathbb{D}} \longrightarrow \mathbb{C}$ be a continuous function which is holomorphic on $\mathbb{D}$. For all $z=r e^{i \theta} \in \mathbb{D}$, we have

$$
u(z)=\frac{1}{2 \pi} \int_{0}^{2 \pi} u\left(e^{i t}\right) \frac{1-r^{2}}{1-2 r \cos (t-\theta)+r^{2}} d t
$$

Proof. Note that for $|z|<R<1$, we have that the map $w \longmapsto \frac{u(w)}{w-\frac{R^{2}}{\bar{z}}}$ is holomorphic on the disk $D_{R} \doteq\{z \in \mathbb{C}:|z|<R\}$. Thus, $\int_{\partial D_{R}} \frac{u(w)}{w-\frac{R^{2}}{\bar{z}}} d w=0$. By the Cauchy's Integral Formula, we have for $|z|<R$

$$
\begin{aligned}
u(z) & =\frac{1}{2 \pi i} \int_{\partial D_{R}} \frac{u(w)}{w-z} d w=\frac{1}{2 \pi i} \int_{\partial D_{R}} u(w)\left(\frac{1}{w-z}+\frac{1}{\frac{w \bar{w}}{\bar{z}}-w}\right) d w \\
& =\frac{1}{2 \pi i} \int_{\partial D_{R}} u(w) \frac{w \bar{w}-z \bar{z}}{w(w-z)(\bar{w}-\bar{z})} d w
\end{aligned}
$$


Considering the change of variable $w=R e^{i t}, t \in[0,2 \pi), d w=i R e^{i t} d t$, we have for $z=r e^{i \theta} \in \mathbb{D}$,

$$
u(z)=\frac{1}{2 \pi i} \int_{\partial D_{R}} u(w) \frac{w \bar{w}-z \bar{z}}{w(w-z)(\bar{w}-\bar{z})} d w=\frac{1}{2 \pi} \int_{0}^{2 \pi} u\left(R e^{i t}\right) \frac{R^{2}-r^{2}}{R^{2}-2 R r \cos (\theta-t)+r^{2}} d t
$$

Taking the limit $R \rightarrow 1^{-}$and applying the Lebesgue's Dominated Convergence Theorem (Theorem 1.2.1), we have the desired result.

Proposition 1.5.8. Let $v: \overline{\mathbb{S}} \longrightarrow \mathbb{C}$ be a continuous function which is holomorphic on $\mathbb{S}$. For all $z=r+i s \in \mathbb{S}$, we have

$v(z)=\int_{\mathbb{R}} v(i t) \frac{e^{-\pi s} \sin (\pi r)}{\sin ^{2}(\pi r)+\left(\cos (\pi r)-e^{-\pi(t-s)}\right)^{2}} d t+\int_{\mathbb{R}} v(1+i t) \frac{e^{-\pi s} \sin (\pi r)}{\sin ^{2}(\pi r)+\left(\cos (\pi r)-e^{i \pi-\pi(t-s)}\right)^{2}} d t$

Proof. Consider the conformal mapping

$$
\begin{aligned}
\Lambda: & \mathbb{S} \longrightarrow \mathbb{D} \\
& z \longmapsto \frac{1+i e^{i \pi z}}{i+e^{i \pi z}}
\end{aligned}
$$

and apply the above Proposition to $v \circ \Lambda^{-1}: \mathbb{D} \rightarrow \mathbb{C}$.

Remark 1.5.9. Let $f: \overline{\mathbb{D}} \longrightarrow X$ be a continuous function which is holomorphic on $\mathbb{D}$. Then, given $x^{*} \in X^{*}$, we have that $x^{*} \circ f$ is under the conditions of the Proposition 1.5.7, and for all $z=r e^{i \theta} \in \mathbb{D}$ we have

$x^{*}(f(z))=\frac{1}{2 \pi} \int_{0}^{2 \pi} x^{*}\left(f\left(e^{i t}\right)\right) \frac{1-r^{2}}{1-2 r \cos (t-\theta)+r^{2}} d t=x^{*}\left(\frac{1}{2 \pi} \int_{0}^{2 \pi} f\left(e^{i t}\right) \frac{1-r^{2}}{1-2 r \cos (t-\theta)+r^{2}} d t\right)$

where the last equality follows from Hille's Theorem (Theorem 1.5.5). Since (1.11) is valid for all $x^{*} \in X^{*}$, it follows from the Hahn-Banach Theorem (Corollary 1.1.5) that for all $z=r e^{i \theta} \in \mathbb{D}$

$$
f(z)=\frac{1}{2 \pi} \int_{0}^{2 \pi} f\left(e^{i t}\right) \frac{1-r^{2}}{1-2 r \cos (t-\theta)+r^{2}} d t
$$

Similarly to what we considered above, given a continuous function $g: \overline{\mathbb{S}} \longrightarrow X$, holomorphic on $\mathbb{S}$, we have, for all $z=r+i s \in \mathbb{S}$ the equality

$g(z)=\int_{\mathbb{R}} g(i t) \frac{e^{-\pi s} \sin (\pi r)}{\sin ^{2}(\pi r)+\left(\cos (\pi r)-e^{-\pi(t-s)}\right)^{2}} d t+\int_{\mathbb{R}} g(1+i t) \frac{e^{-\pi s} \sin (\pi r)}{\sin ^{2}(\pi r)+\left(\cos (\pi r)-e^{i \pi-\pi(t-s)}\right)^{2}} d t$.

With the notion of Bochner measurability and integrability, we can now consider $L_{p}$ spaces with its elements taking values in a Banach space.

Definition 1.5.10. Let $1 \leq p \leq \infty, X$ be a Banach space and $(\Omega, \mathcal{M}, \mu)$ be a positive measure space. We define the space $L_{p}(\Omega, \mu, X)$, the vector valued $L_{p}$ space, as the space of all equivalence classes 
of Bochner-measurable functions $f: \Omega \longrightarrow X$ such that

$$
\begin{gathered}
\int_{\Omega}\|f(\omega)\|_{X}^{p} d \mu(\omega)<\infty, 1 \leq p<\infty \\
\underset{\omega \in \Omega}{\operatorname{ess} \sup }\|f(\omega)\|_{X}<\infty, p=\infty
\end{gathered}
$$

equipped with the norm

$$
\begin{gathered}
\|f\|_{L_{p}(\Omega, \mu, X)}=\left(\int_{\Omega}\|f(\omega)\|_{X}^{p} d \mu(\omega)\right)^{\frac{1}{p}}, \quad 1 \leq p<\infty \\
\|f\|_{L_{\infty}(\Omega, \mu, X)}=\underset{\omega \in \Omega}{\operatorname{essipup}}\|f(\omega)\|_{X}, \quad p=\infty
\end{gathered}
$$

These spaces are Banach spaces. Moreover, we have the following properties:

Proposition 1.5.11. If $X$ is reflexive, then for $1<p<\infty, L_{p}(\Omega, \mu, X)$ is reflexive.

Proof. See [Phi43], Theorem 5.7.

Proposition 1.5.12. If $X$ is strictly convex, then, for $1<p<\infty, L_{p}(\Omega, \mu, X)$ is strictly convex.

Proof. Suppose $X$ is strictly convex and that $L_{p}(\Omega, \mu, X)$ is not strictly convex. Then, according to Proposition 1.1.11 (b), there are $f, g \in L_{p}(\Omega, \mu, X), f \neq g,\|f\|_{L_{p}(\Omega, \mu, X)}=\|g\|_{L_{p}(\Omega, \mu, X)}=1$ and such that

$$
\left\|\frac{f+g}{2}\right\|_{L_{p}(\Omega, \mu, X)}=1=\frac{\|f\|_{L_{p}(\Omega, \mu, X)}^{p}+\|g\|_{L_{p}(\Omega, \mu, X)}^{p}}{2}
$$

Thus,

$$
\int_{\Omega}\left[\frac{\|f(\omega)\|_{X}^{p}+\|g(\omega)\|_{X}^{p}}{2}-\left\|\frac{f(\omega)+g(\omega)}{2}\right\|_{X}^{p}\right] d \mu=0
$$

Note that, since the function $t \mapsto t^{p}$ is convex, we have

$$
\frac{\|f(\omega)\|_{X}^{p}+\|g(\omega)\|_{X}^{p}}{2}-\left\|\frac{f(\omega)+g(\omega)}{2}\right\|_{X}^{p} \geq 0, \quad \forall \omega \in \Omega
$$

Therefore, it follows from (1.12) that

$$
\frac{\|f(\omega)\|_{X}^{p}+\|g(\omega)\|_{X}^{p}}{2}=\left\|\frac{f(\omega)+g(\omega)}{2}\right\|_{X}^{p}, \text { for almost every } \omega \in \Omega
$$

Hence, from Proposition 1.1.11 (a), we have that $f=g$ and we have a contradiction. Thus, $L_{p}(\Omega, \mu, X)$ is strictly convex.

Proposition 1.5.13. If $X$ is uniformly convex, then, for $1<p<\infty, L_{p}(\Omega, \mu, X)$ is uniformly convex. 
Proof. Let $\epsilon>0$ and consider $f, g \in L_{p}(\Omega, \mu, X)$, such that $\|f\|_{L_{p}(\Omega, \mu, X)}=\|g\|_{L_{p}(\Omega, \mu, X)}=1$, $\|f-g\|_{L_{p}(\Omega, \mu, X)} \geq \epsilon$. Given $\omega \in \Omega$, if $f(\omega)=g(\omega)=0$ we make the convention $\delta_{p}\left(\frac{\|f(\omega)-g(\omega)\|_{X}}{\max \left\{\|f(\omega)\|_{X},\|g(\omega)\|_{X}\right\}}\right)=$ 0 . Thus, since $X$ is uniformly convex, it follows from Proposition 1.1.15 that for all $\omega \in \Omega$

$$
\left\|\frac{f(\omega)+g(\omega)}{2}\right\|_{X}^{p} \leq\left[1-\delta_{p}\left(\frac{\|f(\omega)-g(\omega)\|_{X}}{\max \left\{\|f(\omega)\|_{X},\|g(\omega)\|_{X}\right\}}\right)\right] \frac{1}{2}\left[\|f(\omega)\|_{X}^{p}+\|g(\omega)\|_{X}^{p}\right] .
$$

By integrating equation (1.13), we obtain

$$
\left\|\frac{f+g}{2}\right\|_{L_{p}(\Omega, \mu, X)} \leq 1-C
$$

where

$$
2 C=\int_{\Omega}\left[\delta_{p}\left(\frac{\|f(\omega)-g(\omega)\|_{X}}{\max \left\{\|f(\omega)\|_{X},\|g(\omega)\|_{X}\right\}}\right)\right]\left[\|f(\omega)\|_{X}^{p}+\|g(\omega)\|_{X}^{p}\right] d \mu .
$$

Thus, if we show that $C>0$, we have the desired result. For each $\alpha>0$, consider

$$
I_{\alpha} \doteq\left\{\omega \in \Omega:\|f(\omega)-g(\omega)\|_{X} \leq \alpha \max \left\{\|f(\omega)\|_{X},\|g(\omega)\|_{X}\right\}\right\},
$$

and put $J_{\alpha} \doteq I_{\alpha}^{c}$. Note that for all $\omega \in \Omega$ we have

$$
\max \left\{\|f(\omega)\|_{X},\|g(\omega)\|_{X}\right\}^{p} \leq 2^{p-1}\left(\|f(\omega)\|_{X}^{p}+\|g(\omega)\|_{X}^{p}\right)
$$

thus,

$$
\int_{\Omega} \max \left\{\|f(\omega)\|_{X},\|g(\omega)\|_{X}\right\}^{p} d \mu \leq 2^{p-1}\left(\int_{\Omega}\|f(\omega)\|_{X}^{p} d \mu+\int_{\Omega}\|g(\omega)\|_{X}^{p} d \mu\right) \leq 2^{p-1} 2=2^{p} .
$$

Therefore,

$$
\begin{aligned}
\epsilon^{p} \leq\|f-g\|_{L_{p}(\Omega, \mu, X)}^{p} & =\int_{\Omega}\|f(\omega)-g(\omega)\|_{X}^{p} d \mu=\int_{I_{\alpha}}\|f(\omega)-g(\omega)\|_{X}^{p} d \mu+\int_{J_{\alpha}}\|f(\omega)-g(\omega)\|_{X}^{p} d \mu \\
& \leq \alpha^{p} \int_{I_{\alpha}} \max \left\{\|f(\omega)\|_{X},\|g(\omega)\|_{X}\right\}^{p} d \mu+\int_{J_{\alpha}}\|f(\omega)-g(\omega)\|_{X}^{p} d \mu \\
& \leq \alpha^{p} 2^{p}+2^{p} \int_{J_{\alpha}} \max \left\{\|f(\omega)\|_{X},\|g(\omega)\|_{X}\right\}^{p} d \mu
\end{aligned}
$$

Now, consider $\alpha^{p} \doteq \frac{\epsilon^{p}}{2^{p+1}}$, it follows then from (1.14) that

$$
\int_{J_{\alpha}} \max \left\{\|f(\omega)\|_{X},\|g(\omega)\|_{X}\right\}^{p} d \mu \geq \frac{\epsilon^{p}}{2}
$$

Since for all $\omega \in J_{\alpha}$ we have $\frac{\|f(\omega)-g(\omega)\|_{X}}{\max \left\{\|f(\omega)\|_{X},\|g(\omega)\|_{X}\right\}} \geq \alpha$, it follows that

$$
\begin{aligned}
2 C & =\int_{\Omega}\left[\delta_{p}\left(\frac{\|f(\omega)-g(\omega)\|_{X}}{\max \left\{\|f(\omega)\|_{X},\|g(\omega)\|_{X}\right\}}\right)\right]\left[\|f(\omega)\|_{X}^{p}+\|g(\omega)\|_{X}^{p}\right] d \mu \\
& \geq \int_{J_{\alpha}}\left[\delta_{p}\left(\frac{\|f(\omega)-g(\omega)\|_{X}}{\max \left\{\|f(\omega)\|_{X},\|g(\omega)\|_{X}\right\}}\right)\right]\left[\|f(\omega)\|_{X}^{p}+\|g(\omega)\|_{X}^{p}\right] d \mu \\
& \geq \delta_{p}(\alpha) \int_{J_{\alpha}} \max \left\{\|f(\omega)\|_{X},\|g(\omega)\|_{X}\right\}^{p} d \mu \geq \delta_{p}\left(\frac{\epsilon^{p}}{2^{p+1}}\right) \frac{\epsilon^{p}}{2}>0 .
\end{aligned}
$$


We denote by $C_{c}(\Omega)$ the set of continuous compactly supported functions on $\Omega \subseteq \mathbb{R}$.

Proposition 1.5.14. Let $\Omega \subseteq \mathbb{R}$ open and $m$ the Lebesgue measure in $\mathbb{R}$. The set

$$
\left\{\varphi=\sum_{j=1}^{N} \varphi_{j} x_{j}: N \in \mathbb{N}, \varphi_{j} \in C_{c}(\Omega), x_{j} \in X, \forall j=1, \ldots, N\right\}
$$

is dense in $L_{p}(\Omega, m, X)$.

Proof. See [Pis16], Proposition 1.2 and Remark 1.4.

In what follows, let $m$ be the normalized Lebesgue measure on $\mathbb{T}=\partial \mathbb{D}$. For each $z=r e^{i \theta} \in \mathbb{D}$, and $t \in \mathbb{T}$ we denote

$$
d \nu_{z}(t)=\frac{1-r^{2}}{1-2 r \cos (t-\theta)+r^{2}} d m(t) .
$$

Definition 1.5.15. Let $1 \leq p \leq \infty$ and $X$ be a Banach space. We define $H^{p}(X)$ as the space of all functions $g: \overline{\mathbb{D}} \longrightarrow X$ such that $g$ is holomorphic on $\mathbb{D}$, the mapping $t \mapsto g\left(e^{i t}\right)$ is measurable and $g(z)=\int_{\mathbb{T}} g\left(e^{i t}\right) d \nu_{z}(t)$, for all $z \in \mathbb{D}$, equipped with the norm

$$
\begin{gathered}
\|g\|_{H^{p}(X)}^{p}=\int_{\mathbb{T}}\left\|g\left(e^{i t}\right)\right\|_{X}^{p} d m(t), 1 \leq p<\infty, \\
\|g\|_{H^{\infty}(X)}=\underset{\mathbb{T}}{\operatorname{ess} \sup }\left\|g\left(e^{i t}\right)\right\|_{X}, p=\infty .
\end{gathered}
$$

Proposition 1.5.16. For $1 \leq p \leq \infty, H^{p}(X)$ is a Banach space.

Proof. Consider

$$
\begin{aligned}
\Upsilon: H^{p}(X) & \longrightarrow L_{p}(\mathbb{T}, m, X) \\
g & \longmapsto g \uparrow_{\mathbb{T}}
\end{aligned}
$$

Note that $\Upsilon$ is an isometry and

$$
\Upsilon\left(H^{p}(X)\right)=\left\{h \in L_{p}(\mathbb{T}, m, X): z \longmapsto \int_{\mathbb{T}} h\left(e^{i t}\right) d \nu_{z}(t) \text { defines a holomorphic function on } \mathbb{D}\right\} \text {. }
$$

Let us show that $\Upsilon\left(H^{p}(X)\right)$ is closed, from which follows that $H^{p}(X)$ is closed.

Let $\left(\Upsilon\left(f_{n}\right)\right)$ be a sequence in $\Upsilon\left(H^{p}(X)\right)$ such that $\Upsilon\left(f_{n}\right) \longrightarrow h \in L_{p}(\mathbb{T}, m, X)$. We know that the application

$$
\begin{aligned}
\Phi: H^{p}(\mathbb{D}) & \longrightarrow L_{p}(\mathbb{T}) \\
f & \longmapsto f^{*}
\end{aligned}
$$


where $f^{*}$ denotes the radial limit of $f$, is an isometry and $\Phi\left(H^{p}(\mathbb{D})\right)$ is closed.

Let $x^{*} \in X^{*}$. Note that $x^{*} \circ \Upsilon\left(f_{n}\right) \in \Phi\left(H^{p}(\mathbb{D})\right)$, which implies that $x^{*} \circ h \in \Phi\left(H^{p}(\mathbb{D})\right)$. Therefore,

$$
z \longmapsto \int_{\mathbb{T}} x^{*}\left(h\left(e^{i t}\right)\right) d \nu_{z}(t)=x^{*}\left(\int_{\mathbb{T}} h\left(e^{i t}\right) d \nu_{z}(t)\right)
$$

defines a holomorphic function on $\mathbb{D}$ for all $x^{*} \in X^{*}$. Thus, $z \longmapsto \int_{\mathbb{T}} h\left(e^{i t}\right) d \nu_{z}(t)$ defines a holomorphic function on $\mathbb{D}$, i.e., $h \in \Upsilon\left(H^{p}(X)\right)$. 


\section{Chapter 2}

\section{The Complex Method of Interpolation}

Let $A_{0}$ and $A_{1}$ be two Banach spaces. The idea behind an interpolation space $A_{\theta}$ (where $0<\theta<1$ ) is of being an intermediate space between $A_{0}$ and $A_{1}$.

$$
\begin{aligned}
& 0<\theta<1
\end{aligned}
$$

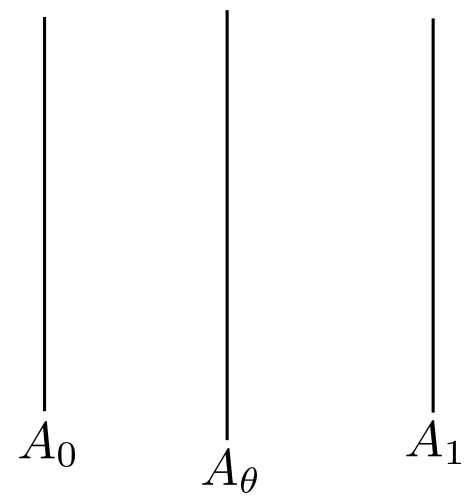

In this chapter, we aim to present one of the motivations and the description of the Complex Interpolation Method of A. Calderón [Cal64].

We start by presenting the Riesz-Thorin Interpolation Theorem, that states the following: for $1<$ $q<p<r<\infty$, if $T$ is an operator on $L_{q}+L_{r}$ that is bounded on both $L_{q}$ and $L_{r}$, then it is also bounded on $L_{p}$. The Complex Interpolation Method can be seen as a generalization of this theorem. Section 2.2 is devoted to formalizing the intuitive ideas of intermediate and interpolation spaces in an abstract context. We also present some properties that will be useful later on.

In Section 2.3 we present the description of the complex interpolation method of A. Calderón. In the last sections of this chapter, we present examples to illustrate the complex method of interpolation. The main references used in this chapter are [BL12], [KS02], [Lun18] and [BS88]. 


\subsection{Riesz-Thorin Interpolation Theorem}

In the following, we will denote by $\mathbb{S}$ the strip in the complex plane given by

$$
\mathbb{S}=\{z \in \mathbb{C}: 0<\mathfrak{R} z<1\}
$$

Recall that the sign of $z=r e^{i \theta} \in \mathbb{C}$ is given by $\operatorname{sgn} z=e^{i \theta}$.

Lemma 2.1.1. (Three Line Theorem): Consider $F: \overline{\mathbb{S}} \longrightarrow \mathbb{C}$ a continuous and bounded function, and holomorphic on $\mathbb{S}$. If for all $t \in \mathbb{R}$ we have that $|F(i t)| \leq M_{0}$ and $|F(1+i t)| \leq M_{1}$ then $|F(\theta+i t)| \leq M_{0}^{1-\theta} M_{1}^{\theta}$ for all $t \in \mathbb{R}, 0<\theta<1$.

Proof. Let $n \in \mathbb{N}$ and $\lambda \in \mathbb{R}$. For each $z=\theta+i t \in \overline{\mathbb{S}}$ define

$$
F_{n}(z)=e^{z^{2} / n+\lambda z} F(z)=F(\theta+i t) e^{\left(\theta^{2}-t^{2}\right) / n+\lambda \theta} e^{2 i \theta t / n+\lambda i t}
$$

Note that $F_{n}$ is holomorphic on $\mathbb{S}$ and continuous on $\overline{\mathbb{S}}$. Moreover, $F_{n}(\theta+i t) \rightarrow 0$ when $t \rightarrow \pm \infty$, and for all $t \in \mathbb{R}$, we have

$$
\begin{gathered}
\left|F_{n}(i t)\right|=|F(i t)|\left|e^{-t^{2} / n+\lambda i t}\right|=|F(i t)| e^{-t^{2} / n} \leq M_{0} \\
\left|F_{n}(1+i t)\right|=|F(1+i t)| e^{\left(1-t^{2}\right) / n+\lambda}=|F(1+i t)| e^{1 / n+\lambda} e^{-t^{2} / n} \leq e^{1 / n+\lambda} M_{1}
\end{gathered}
$$

Therefore $F_{n}$ is bounded on $\overline{\mathbb{S}}$. It follows from the Phragmén-Lindelöf Maximum Principle (Lemma 1.4.6) that $\left|F_{n}(z)\right| \leq \max \left\{M_{0}, M_{1} e^{1 / n+\lambda}\right\}, \forall z \in \overline{\mathbb{S}}$. Then, for all $0<\theta<1$ and for all $t \in \mathbb{R}$

$$
\left|F_{n}(\theta+i t)\right|=|F(\theta+i t)| e^{\left(\theta^{2}-t^{2}\right) / n+\lambda \theta} \leq \max \left\{M_{0}, M_{1} e^{1 / n+\lambda}\right\} .
$$

Consequently,

$$
|F(\theta+i t)| \leq e^{-\left(\theta^{2}-t^{2}\right) / n} e^{-\lambda \theta} \max \left\{M_{0}, M_{1} e^{1 / n+\lambda}\right\} .
$$

Fixing $\theta$ and $t$ and taking $n \rightarrow \infty$ in (2.1), we have that $|F(\theta+i t)| \leq e^{-\theta \lambda} \max \left\{M_{0}, M_{1} e^{\lambda}\right\}=$ $\left(e^{\lambda}\right)^{-\theta} \max \left\{M_{0}, M_{1} e^{\lambda}\right\}$. Putting $e^{\lambda}=\frac{M_{0}}{M_{1}}$, it follows that for $0<\theta<1$ and $t \in \mathbb{R}$

$$
|F(\theta+i t)| \leq\left(\frac{M_{0}}{M_{1}}\right)^{-\theta} \max \left\{M_{0}, M_{1} \frac{M_{0}}{M_{1}}\right\}=M_{0}^{1-\theta} M_{1}^{\theta} .
$$

The idea of the proof of the Riesz-Thorin Theorem will be creating an auxiliary function that satisfies the Three Line Theorem.

Theorem 2.1.2. (Riesz-Thorin Interpolation Theorem) Let $(U, \mathcal{M}, \mu)$ and $(V, \mathcal{N}, \nu)$ be positive measure spaces, $1 \leq p_{0}, p_{1}, q_{0}, q_{1}<\infty$ with $p_{0} \neq p_{1}$ and $q_{0} \neq q_{1}$. Suppose that

(a) $T: L_{p_{0}}(U, \mu) \longrightarrow L_{q_{0}}(V, \nu)$ is a bounded operator with norm $M_{0}$. 
(b) $T: L_{p_{1}}(U, \mu) \longrightarrow L_{q_{1}}(V, \nu)$ is a bounded operator with norm $M_{1}$.

If $0<\theta<1$ and $1 \leq p, q<\infty$ are such that

$$
\frac{1}{p}=\frac{1-\theta}{p_{0}}+\frac{\theta}{p_{1}}, \quad \frac{1}{q}=\frac{1-\theta}{q_{0}}+\frac{\theta}{q_{1}}
$$

then $T: L_{p}(U, \mu) \longrightarrow L_{q}(V, \nu)$ is a bounded operator with norm $M \leq M_{0}^{1-\theta} M_{1}^{\theta}$.

Proof. Denote by $q^{\prime}$ the conjugate exponent of $q$. Consider

$$
\begin{aligned}
& \mathcal{D}_{U}=\left\{f=\sum_{n=1}^{m} \alpha_{n} \chi_{F_{n}}: f \text { is a simple function, } m \in \mathbb{N}, F_{n} \in \mathcal{M}, \mu\left(F_{n}\right)<\infty, n=1, \ldots, m\right\}, \\
& \mathcal{D}_{V}=\left\{f=\sum_{n=1}^{m} \alpha_{n} \chi_{E_{n}}: f \text { is a simple function, } m \in \mathbb{N}, E_{n} \in \mathcal{N}, \nu\left(E_{n}\right)<\infty, n=1, \ldots, m\right\} .
\end{aligned}
$$

We have from Theorem 1.2.9 that

$$
M=\sup \left\{\|T f\|_{L_{q}}: f \in L_{p},\|f\|_{L_{p}}=1\right\}=\sup \left\{\left|\int(T f) g\right|: f \in L_{p}, g \in \mathcal{D}_{V},\|f\|_{L_{p}}=\|g\|_{L_{q^{\prime}}}=1\right\} .
$$

Thus, let $f \in L_{p}$ with $\|f\|_{L_{p}}=1$. It is enough to show that

$$
\left|\int_{V}(T f) g d \nu\right| \leq M_{0}^{1-\theta} M_{1}^{\theta}
$$

with $g \in \mathcal{D}_{V}$ such that $\|g\|_{L_{q^{\prime}}}=1$. We will prove the result for $f \in \mathcal{D}_{U}$. We know from Proposition 1.2.6 that $\mathcal{D}_{U}$ is dense in $L_{p}$, therefore, the general result follows from Lebesgue's Dominated Convergence Theorem (Theorem 1.2.1).

Suppose that $f=\sum_{i=1}^{n} a_{i} \chi_{A_{i}}, g=\sum_{j=1}^{m} b_{j} \chi_{B_{j}}$, with $a_{i}, b_{j} \in \mathbb{C}, \mu\left(A_{i}\right)<\infty, \nu\left(B_{j}\right)<\infty$ for all $i=$ $1, \ldots, n, j=1, \ldots, m, A_{i} \cap A_{k}=\varnothing$ if $i \neq k$ e $B_{j} \cap B_{l}=\varnothing$ if $j \neq l$.

For each $z \in \mathbb{C}$, consider

$$
\alpha(z)=\frac{1-z}{p_{0}}+\frac{z}{p_{1}}, \quad \beta(z)=\frac{1-z}{q_{0}}+\frac{z}{q_{1}} .
$$

Note that $\alpha(\theta)=\frac{1}{p}$ e $\beta(\theta)=\frac{1}{q}$. Now, for each $z \in \overline{\mathbb{S}}$, define

$$
\begin{gathered}
f_{z}(x)=\operatorname{sgn}(f(x))|f(x)|^{\frac{\alpha(z)}{\alpha(\theta)}}, \quad \forall x \in U, \\
g_{z}(y)=\operatorname{sgn}(g(y))|g(y)|^{\frac{1-\beta(z)}{1-\beta(\theta)}}, \quad \forall y \in V .
\end{gathered}
$$

Or more explicitly, considering that $A_{k}$ and $B_{j}$ are pairwise disjoint,

$$
\begin{aligned}
& f_{z}(x)=\operatorname{sgn}\left(\sum_{k=1}^{n} a_{k} \chi_{A_{k}}(x)\right)\left|\sum_{k=1}^{n} a_{k} \chi_{A_{k}}(x)\right|^{\frac{\alpha(z)}{\alpha(\theta)}}=\sum_{k=1}^{n} \operatorname{sgn}\left(a_{k}\right)\left|a_{k}\right|^{\frac{\alpha(z)}{\alpha(\theta)}} \chi_{A_{k}} \\
& g_{z}(y)=\operatorname{sgn}\left(\sum_{k=1}^{m} b_{k} \chi_{B_{k}}(x)\right)\left|\sum_{j=1}^{m} b_{j} \chi_{B_{j}}(y)\right|^{\frac{1-\beta(z)}{1-\beta(\theta)}}=\sum_{j=1}^{m} \operatorname{sgn}\left(b_{j}\right)\left|b_{j}\right|^{\frac{1-\beta(z)}{1-\beta(\theta)}} \chi_{B_{j}}
\end{aligned}
$$


Note that for each $z \in \overline{\mathbb{S}}, f_{z} \in \mathcal{D}_{U}$ and $g_{z} \in \mathcal{D}_{V}$; therefore, $f_{z} \in L_{p_{0}} \cap L_{p_{1}}$, from which follows, together with the hypothesis of the theorem, that $T f_{z} \in L_{q_{0}} \cap L_{q_{1}}$; we also have that $g_{z} \in L_{q_{0}} \cap L_{q_{1}}$.

Thus, we can consider

$$
F(z)=\int_{V}\left(T f_{z}\right) g_{z} d \nu, \forall z \in \overline{\mathbb{S}} .
$$

Let us show that $F$ satisfies the hypothesis of the Three Line Theorem (Lemma 2.1.1):

(i) $F$ is holomorphic on $\mathbb{S}$ :

$$
\begin{aligned}
F(z) & =\int_{V}\left(T f_{z}\right) g_{z} d \nu=\int_{V} T\left(\sum_{k=1}^{n} \operatorname{sgn}\left(a_{k}\right)\left|a_{k}\right|^{\frac{\alpha(z)}{\alpha(\theta)}} \chi_{A_{k}}\right)\left(\sum_{j=1}^{m} \operatorname{sgn}\left(b_{j}\right)\left|b_{j}\right|^{\frac{1-\beta(z)}{1-\beta(\theta)}} \chi_{B_{j}}\right) d \nu \\
& =\int_{V}\left(\sum_{k=1}^{n} \operatorname{sgn}\left(a_{k}\right)\left|a_{k}\right|^{\frac{\alpha(z)}{\alpha(\theta)}} T\left(\chi_{A_{k}}\right)\right)\left(\sum_{j=1}^{m} \operatorname{sgn}\left(b_{j}\right)\left|b_{j}\right|^{\frac{1-\beta(z)}{1-\beta(\theta)}} \chi_{B_{j}}\right) d \nu \\
& =\sum_{k=1}^{n} \sum_{j=1}^{m} \operatorname{sgn}\left(a_{k}\right) \operatorname{sgn}\left(b_{j}\right)\left|a_{k}\right|^{\frac{\alpha(z)}{\alpha(\theta)}}\left|b_{j}\right|^{\frac{1-\beta(z)}{1-\beta(\theta)}} \int_{V} T\left(\chi_{A_{k}}\right) \chi_{B_{j}} d \nu
\end{aligned}
$$

Note that $\alpha$ and $\beta$ are holomorphic functions, thus, since $F$ is a linear combination of holomorphic functions, it follows that $F$ is holomorphic on $\mathbb{S}$.

(ii) $F$ is bounded on $\overline{\mathbb{S}}$ : If $z=s+i t \in \overline{\mathbb{S}}$, then

$$
\begin{gathered}
\mathfrak{R}\left[\frac{\alpha(z)}{\alpha(\theta)}\right]=\mathfrak{R}\left[\left(\frac{1-s-i t}{p_{0}}+\frac{s+i t}{p_{1}}\right) p\right] \\
\mathfrak{R}\left[\frac{1-\beta(z)}{1-\beta(\theta)}\right]=\mathfrak{R}\left[\frac{1-\left(\frac{1-(s+i t)}{q_{0}}+\frac{s+i t}{q_{1}}\right)}{1-\frac{1}{q}}\right]
\end{gathered}
$$

only depend on $s \in[0,1]$, therefore, $\mathfrak{R}\left[\frac{\alpha(z)}{\alpha(\theta)}\right]$ and $\mathfrak{R}\left[\frac{1-\beta(z)}{1-\beta(\theta)}\right]$ are bounded. If

$$
C=\max \left\{\left|\int_{V} T\left(\chi_{A_{k}}\right) \chi_{B_{j}} d \nu\right|: k=1, \ldots, n, j=1, \ldots, m\right\}
$$

we have that

$$
|F(z)| \leq\left.\left. C \sum_{k=1}^{n} \sum_{j=1}^{m}\left|\operatorname{sgn}\left(a_{k}\right) \operatorname{sgn}\left(b_{j}\right)\right||| a_{k}\right|^{\frac{\alpha(z)}{\alpha(\theta)}}||\left|b_{j}\right|^{\frac{1-\beta(z)}{1-\beta(\theta)}}\left|=C \sum_{k=1}^{n} \sum_{j=1}^{m}\right| a_{k}\right|^{\Re\left[\frac{\alpha(z)}{\alpha(\theta)}\right]}\left|b_{j}\right|^{\Re\left[\frac{1-\beta(z)}{1-\beta(\theta)}\right]}
$$

is also bounded on $\overline{\mathbb{S}}$.

(iii) $|F(i t)| \leq M_{0}$ and $|F(1+i t)| \leq M_{1}$ for all $t \in \mathbb{R}$ : If $q_{0}^{\prime}$ is the conjugate exponent of $q_{0}$, we have by Hölder's Inequality (Theorem 1.2.2) that

$$
\begin{aligned}
|F(i t)| & =\left|\int_{V}\left(T f_{i t}\right) g_{i t} d \nu\right| \leq\left(\int_{V}\left|T f_{i t}\right|^{q_{0}}\right)^{1 / q_{0}}\left(\int_{V}\left|g_{i t}\right|^{q_{0}^{\prime}}\right)^{1 / q_{0}^{\prime}} \\
& =\left\|T f_{i t}\right\|_{L_{q_{0}}}\left\|g_{i t}\right\|_{L_{q_{0}^{\prime}}} \leq M_{0}\left\|f_{i t}\right\|_{L_{p_{0}}}\left\|g_{i t}\right\|_{L_{q_{0}^{\prime}}}
\end{aligned}
$$


Moreover, we have that

$$
\left\|f_{i t}\right\|_{L_{p_{0}}}^{p_{0}}=\left.\left.\int_{U}|\operatorname{sgn}(f(x))| f(x)\right|^{\frac{\alpha(i t)}{\alpha(\theta)}}\right|^{p_{0}} d \mu=\int_{U}|f(x)|^{\Re\left[\frac{\alpha(i t)}{\alpha(\theta)}\right] p_{0}} d \mu=\int_{U}|f(x)|^{\frac{p}{p_{0}} p_{0}} d \mu=\|f\|_{L_{p}}^{p}=1
$$

In the same way, we can show that $\left\|g_{i t}\right\|_{L_{q_{0}^{\prime}}}=1$, then, $|F(i t)| \leq M_{0}$. In a similar way, we can show that $|F(1+i t)| \leq M_{1}$.

Thus, it follows from the Three Line Theorem that $|F(\theta+i t)| \leq M_{0}^{1-\theta} M_{1}^{\theta}, \forall t \in \mathbb{R}$.

Now, note that $f_{\theta}=f$ and $g_{\theta}=g$, then

$$
|F(\theta)|=\left|\int_{V}\left(T f_{\theta}\right) g_{\theta} d \nu\right|=\left|\int_{V}(T f) g d \nu\right| \leq M_{0}^{1-\theta} M_{1}^{\theta} .
$$

\subsection{Interpolation Spaces}

This section is devoted to the abstract theory of interpolation. We consider here the context of normed spaces.

Definition 2.2.1. A topological vector space $\mathfrak{A}$ is a a vector space with a topology $\tau$ such that the vector space operations are continuous with respect to $\tau$.

Definition 2.2.2. Let $X$ and $Y$ be two topological vector spaces. We say that $Y$ is (algebraically and topologically) embedded in $X$ if:

(i) $Y$ is included in $X$.

(ii) The space $X$ induces a structure of vector space in $Y$ that coincides with the structure of $Y$.

(iii) The natural inclusion $i: Y \rightarrow X$ is continuous.

In the context of normed spaces, the above definition is equivalent to saying that $Y$ is a subspace of $X$ and the natural inclusion is continuous. Thus, if $X$ and $Y$ are normed spaces, the condition (iii) is equivalent to

(iii') There is $c \in \mathbb{R}^{+}$such that

$$
\|x\|_{X} \leq c\|x\|_{Y}, \forall x \in Y \text {. }
$$

If $Y$ is embedded in $X$, we set the notation $Y \rightarrow X$.

The intuitive idea of an interpolation space is to be an intermediate space between other spaces. It is natural to demand that they are all embedded in a common space. This motivates the following definition:

Definition 2.2.3. Let $A_{0}$ and $A_{1}$ be two topological vector spaces. We say that $A_{0}$ and $A_{1}$ are compatible (or that the pair $\left(A_{0}, A_{1}\right)$ is compatible) if there is a topological Hausdorff vector 
space $\mathfrak{A}$ such that $A_{0}$ and $A_{1}$ are embedded in $\mathfrak{A}$.

Given two compatible spaces $A_{0}$ and $A_{1}$, we can consider the sum $A_{0}+A_{1}=\left\{a \in \mathfrak{A}: a=a_{0}+a_{1}, a_{0} \epsilon\right.$ $\left.A_{0}, a_{1} \in A_{1}\right\}$ and the intersection $A_{0} \cap A_{1}=\left\{a \in \mathfrak{A}: a \in A_{0}, a \in A_{1}\right\}$ of these spaces.

Lemma 2.2.4. Suppose that $A_{0}$ and $A_{1}$ are compatible normed spaces. Then $A_{0} \cap A_{1}$ is a normed space with the norm given by

$$
\|a\|_{A_{0} \cap A_{1}}=\max \left\{\|a\|_{A_{0}},\|a\|_{A_{1}}\right\}
$$

And $A_{0}+A_{1}$ is a normed space with the norm

$$
\|a\|_{A_{0}+A_{1}}=\inf \left\{\left\|a_{0}\right\|_{A_{0}}+\left\|a_{1}\right\|_{A_{1}}: a=a_{0}+a_{1}, a_{0} \in A_{0}, a_{1} \in A_{1}\right\} .
$$

Moreover, if $A_{0}$ and $A_{1}$ are Banach spaces, then $A_{0} \cap A_{1}$ and $A_{0}+A_{1}$ are Banach spaces.

Proof. It is immediate that (2.2) and (2.3) represent norms in the respective spaces (it follows from the fact that $\|\cdot\|_{A_{0}}$ and $\|\cdot\|_{A_{1}}$ are norms). Let us see that if $A_{0}$ and $A_{1}$ are Banach spaces, then $A_{0} \cap A_{1}$ and $A_{0}+A_{1}$ are also complete.

(i) $A_{0} \cap A_{1}$ is complete: Let $\epsilon>0$ and $\left(a_{n}\right)$ be a Cauchy sequence in $A_{0} \cap A_{1}$. Then there is $n_{0} \in \mathbb{N}$ such that $\left\|a_{n}-a_{m}\right\|_{A_{0} \cap A_{1}}<\epsilon, \forall n, m \geq n_{0}$. Thus, for $j=0,1$, we have

$$
\left\|a_{n}-a_{m}\right\|_{A_{j}} \leq\left\|a_{n}-a_{m}\right\|_{A_{0} \cap A_{1}}<\epsilon, \forall n, m \geq n_{0},
$$

that is, $\left(a_{n}\right)$ is a Cauchy sequence in $A_{j}$ (which is complete) and therefore there are $a^{j} \in A_{j}$ such that $a_{n} \rightarrow a^{j}$ in $A_{j}$, for $j=0,1$. Since $A_{0}$ and $A_{1}$ are embedded in a Hausdorff space $\mathfrak{A}$, it follows that $a_{n} \rightarrow a^{j}$ in $\mathfrak{A}$, and by the uniqueness of the limit in $\mathfrak{A}$, we have that $a^{0}=a^{1} \in A_{0} \cap A_{1}$. Thus, the Cauchy sequence $\left(a_{n}\right)$ is convergent in $A_{0} \cap A_{1}$.

(ii) $A_{0}+A_{1}$ is complete: Consider $\left(a_{n}\right)$ a sequence in $A_{0}+A_{1}$ such that $\sum_{n}\left\|a_{n}\right\|_{A_{0}+A_{1}}<\infty$. Let us show that the series $\sum_{n} a_{n}$ is convergent in $A_{0}+A_{1}$. For each $n \in \mathbb{N}$ we know that

$$
\left\|a_{n}\right\|_{A_{0}+A_{1}}=\inf \left\{\left\|a_{n}^{0}\right\|_{A_{0}}+\left\|a_{n}^{1}\right\|_{A_{1}}: a_{n}=a_{n}^{0}+a_{n}^{1}, a_{n}^{0} \in A_{0}, a_{n}^{1} \in A_{1}\right\} .
$$

By the infimum property, for every $\epsilon>0$ we can find a decomposition $a_{n}=a_{n}^{0}+a_{n}^{1}$ such that

$$
\left\|a_{n}^{0}\right\|_{A_{0}}+\left\|a_{n}^{1}\right\|_{A_{1}} \leq\left\|a_{n}\right\|_{A_{0}+A_{1}}+\epsilon
$$

In particular, for $\epsilon=\left\|a_{n}\right\|_{A_{0}+A_{1}}$, we have that

$$
\left\|a_{n}^{0}\right\|_{A_{0}}+\left\|a_{n}^{1}\right\|_{A_{1}} \leq 2\left\|a_{n}\right\|_{A_{0}+A_{1}}
$$

(note that if $\left\|a_{n}\right\|_{A_{0}+A_{1}}=0$, the above inequality it is still valid). Thus, $\sum_{n}\left\|a_{n}^{0}\right\|_{A_{0}}<\infty$ and $\sum_{n}\left\|a_{n}^{1}\right\|_{A_{1}}<\infty$. Since both $A_{0}$ and $A_{1}$ are complete, there are $a^{0} \in A_{0}$ and $a^{1} \in A_{1}$ such that $a^{0}=\sum_{n} a_{n}^{0}$ and $a^{1}=\sum_{n} a_{n}^{1}$. Putting $a=a^{0}+a^{1}$, let us show that $a=\sum_{n} a_{n} \in A_{0}+A_{1}$. Indeed, 
given $\epsilon>0$, there is $n_{0} \in \mathbb{N}$ such that

$$
\left\|a^{j}-\sum_{n=1}^{m} a_{n}^{j}\right\|_{A_{j}}<\frac{\epsilon}{2}, \forall m \geq n_{0}, j=0,1
$$

Thus, for all $m \geq n_{0}$, we have that

$$
\left\|a-\sum_{n=1}^{m} a_{n}\right\|_{A_{0}+A_{1}} \leq\left\|a^{0}-\sum_{n=1}^{m} a_{n}^{0}\right\|_{A_{0}}+\left\|a^{1}-\sum_{n=1}^{m} a_{n}^{1}\right\|_{A_{1}}<\epsilon .
$$

Whenever we consider the sum and intersection spaces, we will be considering the norms as given in the above lemma.

Given $A$ and $B$ vector spaces, $C$ subspace of $A$ and $T: A \longrightarrow B$ an operator, we denote by $T_{C}$ the restriction of $T$ to $C$.

Definition 2.2.5. Let $\left(A_{0}, A_{1}\right)$ and $\left(B_{0}, B_{1}\right)$ be two normed compatible couples, and $A$ and $B$ be normed spaces.

(a) We say that $A$ is an intermediate space between $A_{0}$ and $A_{1}$ (or with respect to $\left(A_{0}, A_{1}\right)$ ) if

$$
A_{0} \cap A_{1} \hookrightarrow A \hookrightarrow A_{0}+A_{1}
$$

(b) $A$ is said to be an interpolated space with respect to $\left(A_{0}, A_{1}\right)$ if $A$ is an intermediate space with respect to $\left(A_{0}, A_{1}\right)$ and if

$$
T \in \mathcal{B}\left(A_{0}+A_{1}\right) \text { such that } T_{A_{j}} \in \mathcal{B}\left(A_{j}\right), j=0,1 \text { implies that } T_{A} \in \mathcal{B}(A) \text {. }
$$

(c) $A$ and $B$ are called interpolated spaces with respect to $\left(A_{0}, A_{1}\right)$ and $\left(B_{0}, B_{1}\right)$ if $A$ and $B$ are intermediate spaces with respect to $\left(A_{0}, A_{1}\right)$ and $\left(B_{0}, B_{1}\right)$ respectively, and if

$$
T \in \mathcal{B}\left(A_{0}+A_{1}, B_{0}+B_{1}\right) \text { such that } T_{A_{j}} \in \mathcal{B}\left(A_{j}, B_{j}\right), j=0,1 \text { implies that } T_{A} \in \mathcal{B}(A, B) \text {. }
$$

Example 2.2.6. If $1 \leq p_{0}<p<p_{1} \leq \infty$ then $L_{p}$ is an interpolated space between $L_{p_{0}}$ and $L_{p_{1}}$. Let $0<\theta<1$ be such that $\frac{1}{p}=\frac{1-\theta}{p_{0}}+\frac{\theta}{p_{1}}$. According to Propositions 1.2.7 and 1.2.8 we have that

$$
L_{p_{0}} \cap L_{p_{1}} \subset L_{p} \subset L_{p_{0}}+L_{p_{1}}
$$

Moreover, if $f \in L_{p_{0}} \cap L_{p_{1}}$, then

$$
\begin{aligned}
\|f\|_{L_{p}} & \leq\|f\|_{L_{p_{0}}}^{\theta}\|f\|_{L_{p_{1}}}^{1-\theta} \leq \max \left\{\|f\|_{L_{p_{0}}}^{\theta}\|f\|_{L_{p_{0}}}^{1-\theta},\|f\|_{L_{p_{1}}}^{\theta}\|f\|_{L_{p_{1}}}^{1-\theta}\right\} \\
& =\max \left\{\|f\|_{L_{p_{0}}},\|f\|_{L_{p_{1}}}\right\}=\|f\|_{L_{p_{0}} \cap L_{p_{1}}}
\end{aligned}
$$


Now, if $f \in L_{p}$, taking $g$ and $h$ as in the proof of Proposition 1.2.7 we have

$$
\begin{aligned}
\|f\|_{L_{p_{0}}+L_{p_{1}}} & \leq\|g\|_{L_{p_{0}}}+\|h\|_{L_{p_{1}}}=\left(\int|g|^{p_{0}}\right)^{1 / p_{0}}+\left(\int|h|^{p_{1}}\right)^{1 / p_{1}} \\
& \leq\left(\int|f|^{p}\right)^{1 / p_{0}}+\left(\int|f|^{p}\right)^{1 / p_{1}}=\|f\|_{L_{p}}^{p / p_{0}}+\|f\|_{L_{p}}^{p / p_{1}}
\end{aligned}
$$

Suppose first that $\|f\|_{L_{p}}=1$, then, from (2.4),

$$
\|f\|_{L_{p_{0}}+L_{p_{1}}} \leq\|f\|_{L_{p}}^{p / p_{0}}+\|f\|_{L_{p}}^{p / p_{1}}=2=2\|f\|_{L_{p}}
$$

Now, if $f \in L_{p}$ is arbitrary, consider $g=\frac{f}{\|f\|_{L_{p}}} \in L_{p}$. Thus $\|g\|_{L_{p}}=1$ and by (2.5) it follows that

$$
\|g\|_{L_{p_{0}}+L_{p_{1}}}=\frac{\|f\|_{L_{p_{0}}+L_{p_{1}}}}{\|f\|_{L_{p}}} \leq 2\|g\|_{L_{p}}=\frac{2\|f\|_{L_{p}}}{\|f\|_{L_{p}}}
$$

that is, we have $\|f\|_{L_{p_{0}}+L_{p_{1}}} \leq 2\|f\|_{L_{p}}$.

Therefore, the inclusions $L_{p_{0}} \cap L_{p_{1}} \subseteq L_{p} \subseteq L_{p_{0}}+L_{p_{1}}$ are continuous and $L_{p}$ is an intermediate space between $L_{p_{0}}$ and $L_{p_{1}}$.

By the Riesz-Thorin Theorem (2.1.2), it follows that $L_{p}$ is an interpolated space with respect to $\left(L_{p_{0}}, L_{p_{1}}\right)$.

Example 2.2.7. $A_{0} \cap A_{1}$ and $B_{0} \cap B_{1}$ are interpolated spaces with respect to $\left(A_{0}, A_{1}\right)$ and $\left(B_{0}, B_{1}\right)$. The same is valid for $A_{0}+A_{1}$ and $B_{0}+B_{1}$.

If $a \in A_{0} \cap A_{1}$, then $\|a\|_{A_{0}+A_{1}} \leq\|a\|_{A_{j}}, j=0,1$. Thus,

$$
\|a\|_{A_{0}+A_{1}} \leq \max \left\{\|a\|_{A_{0}},\|a\|_{A_{1}}\right\}=\|a\|_{A_{0} \cap A_{1}}
$$

so the inclusion $A_{0} \cap A_{1} \hookrightarrow A_{0}+A_{1}$ is continuous and we have that $A_{0} \cap A_{1}$ is an intermediate space with respect to $\left(A_{0}, A_{1}\right)$ and $A_{0}+A_{1}$ is an intermediate space with respect to $\left(A_{0}, A_{1}\right)$.

Now, consider $T \in \mathcal{B}\left(A_{0}+A_{1}, B_{0}+B_{1}\right)$ such that $T_{A_{j}} \in \mathcal{B}\left(A_{j}, B_{j}\right), j=0,1$. If $a \in A_{0} \cap A_{1}$ it is immediate that $T(a) \in B_{0} \cap B_{1}$. Thus, $T_{A} \in \mathcal{B}(A, B)$; therefore, $A_{0} \cap A_{1}$ and $B_{0} \cap B_{1}$ are interpolated spaces with respect to $\left(A_{0}, A_{1}\right)$ and $\left(B_{0}, B_{1}\right)$, and the same is valid for $A_{0}+A_{1}$ and $B_{0}+B_{1}$.

We will now present a result involving duality that will be useful later on.

Theorem 2.2.8. Let $\left(A_{0}, A_{1}\right)$ be a compatible Banach couple. If $A_{0} \cap A_{1}$ is dense in both $A_{0}$ and $A_{1}$ then $\left(A_{0}+A_{1}\right)^{*}=A_{0}^{*} \cap A_{1}^{*}$, with equal norms.

Proof. Let $a^{*} \in A_{0}^{*} \cap A_{1}^{*}, a \in A_{0}+A_{1}$ and $\epsilon>0$. Using the infimum property and the definition of 
the norm in $A_{0}+A_{1}$, consider $a_{0} \in A_{0}, a_{1} \in A_{1}$ such that $a=a_{0}+a_{1}$ and

$$
\left\|a_{0}\right\|_{A_{0}}+\left\|a_{1}\right\|_{A_{1}} \leq\|a\|_{A_{0}+A_{1}}+\frac{\epsilon}{\left\|a^{*}\right\|_{A_{0}^{*}+A_{1}^{*}}} .
$$

We have,

$$
\begin{aligned}
\left|a^{*}(a)\right| & \leq\left|a^{*}\left(a_{0}\right)\right|+\left|a^{*}\left(a_{1}\right)\right| \leq\left\|a^{*}\right\|_{A_{0}^{*}}\left\|a_{0}\right\|_{A_{0}}+\left\|a^{*}\right\|_{A_{1}^{*}}\left\|a_{1}\right\|_{A_{1}} \leq \max \left\{\left\|a^{*}\right\|_{A_{0}^{*}},\left\|a^{*}\right\|_{A_{1}^{*}}\right\}\left(\left\|a_{0}\right\|_{A_{0}}+\left\|a_{1}\right\|_{A_{1}}\right) \\
& =\left\|a^{*}\right\|_{A_{0}^{*} \cap A_{1}^{*}}\left(\left\|a_{0}\right\|_{A_{0}}+\left\|a_{1}\right\|_{A_{1}}\right) \leq\left\|a^{*}\right\|_{A_{0}^{*} \cap A_{1}^{*}}\left(\|a\|_{A_{0}+A_{1}}+\frac{\epsilon}{\left\|a^{*}\right\|_{A_{0}^{*}+A_{1}^{*}}}\right)=\left\|a^{*}\right\|_{A_{0}^{*} \cap A_{1}^{*}}\|a\|_{A_{0}+A_{1}}+\epsilon
\end{aligned}
$$

By the arbitrariness of $\epsilon>0$, it follows that $a^{*} \in\left(A_{0}+A_{1}\right)^{*}$ and $\left\|a^{*}\right\|_{\left(A_{0}+A_{1}\right)^{*}} \leq\left\|a^{*}\right\|_{A_{0}^{*} \cap A_{1}^{*}}$.

On the other hand, let $a^{*} \in\left(A_{0}+A_{1}\right)^{*}$. Denote by $b^{*}$ the restriction of $a^{*}$ to $A_{0} \cap A_{1}$. Thus, for every $a \in A_{0} \cap A_{1}$ we have

$$
\left|b^{*}(a)\right|=\left|a^{*}(a)\right| \leq\left\|a^{*}\right\|_{\left(A_{0}+A_{1}\right)^{*}}\|a\|_{A_{0}+A_{1}} \leq\left\|a^{*}\right\|_{\left(A_{0}+A_{1}\right)^{*}}\|a\|_{A_{0} \cap A_{1}},
$$

that is, $b^{*} \in\left(A_{0} \cap A_{1}\right)^{*}$ and $\left\|b^{*}\right\|_{\left(A_{0} \cap A_{1}\right)^{*}} \leq\left\|a^{*}\right\|_{\left(A_{0}+A_{1}\right)^{*}}$. By the Hahn-Banach Theorem (Corollary 1.1.4), there are $b_{j}^{*} \in A_{j}^{*}, j=0,1$, such that

$$
\begin{gathered}
b_{j}^{*}(a)=b^{*}(a)=a^{*}(a), \quad \forall a \in A_{j}, \\
\left\|b_{j}^{*}\right\|_{A_{j}^{*}}=\left\|b^{*}\right\|_{\left(A_{0} \cap A_{1}\right)^{*}}
\end{gathered}
$$

By the density hypothesis, $b_{0}^{*}$ and $b_{1}^{*}$ are determined by their values in $A_{0} \cap A_{1}$, thus,

$$
b_{j}^{*}(a)=a^{*}(a), \forall a \in A_{j},
$$

and it follows that $a^{*} \in A_{0}^{*} \cap A_{1}^{*}$. Moreover,

$$
\left\|a^{*}\right\|_{A_{0}^{*} \cap A_{1}^{*}}=\max \left\{\left\|a^{*}\right\|_{A_{0}^{*}}\left\|a^{*}\right\|_{A_{1}^{*}}\right\}=\left\|b^{*}\right\|_{\left(A_{0} \cap A_{1}\right)^{*}} \leq\left\|a^{*}\right\|_{\left(A_{0}+A_{1}\right)^{*}} .
$$

Under the same hypotheses as the theorem above, it is also valid that $\left(A_{0} \cap A_{1}\right)^{*}=A_{0}^{*}+A_{1}^{*}$ (see [BL12], Theorem 2.7.1, for an elegant proof of this fact).

\subsection{The Complex Method of Interpolation}

The Complex Interpolation Method due to A. Calderón [Cal64] can be seen as a generalization of the Riesz-Thorin Theorem and is mainly based on extending some ideas from the classical theory of complex analysis to the context of functions taking values in Banach spaces.

We reiterate that we will consider all vector spaces over the complex field. Interpolation methods for real spaces also exist and are rather different (see e.g. Real Interpolation Method in [BL12]).

Consider $\bar{A}=\left(A_{0}, A_{1}\right)$ a compatible Banach couple. 
Definition 2.3.1. The space $\mathcal{F}(\bar{A})$ is defined as the set of functions $f: \overline{\mathbb{S}} \longrightarrow A_{0}+A_{1}$ that are bounded and continuous on $\overline{\mathbb{S}}$ and holomorphic on $\mathbb{S}$, such that the functions $t \longmapsto f(j+i t), j=0,1$ are continuous from $\mathbb{R}$ into $A_{j}$, and $\|f(j+i t)\|_{A_{j}} \longrightarrow 0$ when $|t| \rightarrow+\infty(j=0,1)$.

Note that $\mathcal{F}(\bar{A})$ is a vector space. Let us consider on $\mathcal{F}(\bar{A})$ the norm

$$
\|f\|_{\mathcal{F}(\bar{A})}=\max \left\{\sup _{t \in \mathbb{R}}\|f(i t)\|_{A_{0}}, \sup _{t \in \mathbb{R}}\|f(1+i t)\|_{A_{1}}\right\} .
$$

It is immediate that (2.6) satisfies triangular inequality and homogeneity. Let us see that $\|f\|_{\mathcal{F}(\bar{A})}=0$ implies $f=0$.

It follows from the Phragmen-Lindelöf principle for Banach spaces (Theorem 1.4.11) that for all $z \in \overline{\mathbb{S}}$

$$
\|f(z)\|_{A_{0}+A_{1}} \leq \max \left\{\sup _{t \in \mathbb{R}}\|f(i t)\|_{A_{0}+A_{1}}, \sup _{t \in \mathbb{R}}\|f(1+i t)\|_{A_{0}+A_{1}}\right\} .
$$

Moreover, as $\|f(j+i t)\|_{A_{0}+A_{1}} \leq\|f(j+i t)\|_{A_{j}}, j=0,1$, it follows that

$$
\begin{aligned}
\|f(z)\|_{A_{0}+A_{1}} & =\max \left\{\sup _{t \in \mathbb{R}}\|f(i t)\|_{A_{0}+A_{1}}, \sup _{t \in \mathbb{R}}\|f(1+i t)\|_{A_{0}+A_{1}}\right\} \\
& \leq \max \left\{\sup _{t \in \mathbb{R}}\|f(i t)\|_{A_{0}}, \sup _{t \in \mathbb{R}}\|f(1+i t)\|_{A_{1}}\right\}=\|f\|_{\mathcal{F}(\bar{A})} .
\end{aligned}
$$

and therefore (2.6) defines a norm.

Lemma 2.3.2. $\mathcal{F}(\bar{A})$ is a Banach space.

Proof. Let $\left(f_{n}\right)$ be a sequence of functions in $\mathcal{F}(\bar{A})$ such that $\sum_{n}\left\|f_{n}\right\|_{\mathcal{F}}<\infty$. From equation (2.7), we have that $\forall z \in \overline{\mathbb{S}}$

$$
\left\|f_{n}(z)\right\|_{A_{0}+A_{1}} \leq\|f\|_{\mathcal{F}(\bar{A})} .
$$

Therefore, $\sum_{n}\left\|f_{n}(z)\right\|_{A_{0}+A_{1}}<\infty, \forall z \in \overline{\mathbb{S}}$. Since $A_{0}+A_{1}$ is a Banach space (Lemma 2.2.4), we have that for each $z \in \bar{S}, \sum_{n} f_{n}(z)$ is convergent in $A_{0}+A_{1}$. Let us show that the function

$$
\begin{aligned}
f: \bar{S} & \longrightarrow A_{0}+A_{1} \\
z & \longmapsto \sum_{n} f_{n}(z)
\end{aligned}
$$

belongs to $\mathcal{F}(\bar{A})$. Note that for all $z \in \overline{\mathbb{S}}$ and $N \in \mathbb{N}$

$$
\left\|\sum_{n=1}^{N} f_{n}(z)-f(z)\right\|_{A_{0}+A_{1}}=\left\|\sum_{n \geq N} f_{n}(z)\right\|_{A_{0}+A_{1}} \leq \sum_{n \geq N}\left\|f_{n}(z)\right\|_{A_{0}+A_{1}} \leq \sum_{n \geq N}\left\|f_{n}\right\|_{\mathcal{F}(\bar{A})}
$$

Thus, the series $\sum_{n} f_{n}(z)$ converges uniformly on $\overline{\mathbb{S}}$. Therefore, $f$ is bounded and continuous on $\overline{\mathbb{S}}$ and holomorphic on $\mathbb{S}$.

Moreover, for $j=0,1$ and for all $t \in \mathbb{R}$ we have $\left\|f_{n}(j+i t)\right\|_{A_{j}} \leq\left\|f_{n}\right\|_{\mathcal{F}(\bar{A})}$, and therefore, $\sum_{n} f_{n}(j+i t)$ converges uniformly to $g_{j}(t) \in A_{j}$. Besides that, $g_{j}(t)=f(j+i t) \in A_{j} \subset A_{0}+A_{1}$, because for $N \in \mathbb{N}$ 
we have

$$
\begin{aligned}
\left\|g_{j}(t)-f(j+i t)\right\|_{A_{0}+A_{1}} & \leq\left\|\sum_{n=1}^{N} f_{n}(j+i t)-g_{j}(t)\right\|_{A_{0}+A_{1}}+\left\|\sum_{n=1}^{N} f_{n}(j+i t)-f(j+i t)\right\|_{A_{0}+A_{1}} \\
& \leq\left\|\sum_{n=1}^{N} f_{n}(j+i t)-g_{j}(t)\right\|_{A_{j}}+\left\|\sum_{n=1}^{N} f_{n}(j+i t)-f(j+i t)\right\|_{A_{0}+A_{1}}
\end{aligned}
$$

and then $\left\|g_{j}(t)-f(j+i t)\right\|_{A_{0}+A_{1}} \rightarrow 0$ when $N \rightarrow \infty$. Thus, $\sum_{n} f_{n}(j+i t)$ converges uniformly to $f(j+i t)$ in $A_{j}$, and it follows that the functions $t \mapsto f(j+i t)$ are continuous from $\mathbb{R}$ to $A_{j}(j=0,1)$. This uniform convergence also implies that $f(j+i t)$ converges to zero when $|t| \rightarrow \infty$. Therefore, $f \in \mathcal{F}(\bar{A})$ and $\mathcal{F}(\bar{A})$ is a Banach space.

Definition 2.3.3. The complex interpolation space $A_{\theta}=\left(A_{0}, A_{1}\right)_{\theta}$ is defined by the following relation: $a \in A_{\theta}$ if, and only if, there is $f \in \mathcal{F}(\bar{A})$ such that $f(\theta)=a$. The space $A_{\theta}$ is equipped with the norm

$$
\|a\|_{A_{\theta}}=\inf \left\{\|f\|_{\mathcal{F}(\bar{A})}: f(\theta)=a\right\} .
$$

Theorem 2.3.4. $A_{\theta}$ is a Banach space.

Proof. Consider the map

$$
\begin{aligned}
\Psi_{\theta}: \mathcal{F}(\bar{A}) & \longrightarrow A_{0}+A_{1} \\
f & \longmapsto f(\theta)
\end{aligned}
$$

Note that $\Psi_{\theta}$ is linear and it follows from (2.7) that for every $f \in \mathcal{F}(\bar{A}),\|f(\theta)\|_{A_{0}+A_{1}} \leq\|f\|_{\mathcal{F}(\bar{A})}$, i.e. $\Psi_{\theta}$ is bounded. It follows from the continuity of $\Psi_{\theta}$ that $\operatorname{ker} \Psi_{\theta}=\{f \in \mathcal{F}(\bar{A}): f(\theta)=0\}$ is closed. Since $\mathcal{F}(\bar{A})$ is Banach, the quotient space $\mathcal{F}(\bar{A}) / \operatorname{ker} \Psi_{\theta}$ is Banach. Moreover, the map

$$
\begin{aligned}
\Phi_{\theta}: A_{\theta} & \longrightarrow \frac{\mathcal{F}(\bar{A})}{\operatorname{ker} \Psi_{\theta}} \\
a & \longmapsto[f]
\end{aligned}
$$

where $f \in \mathcal{F}(\bar{A})$ is such that $f(\theta)=a$, defines an isometry between $A_{\theta}$ and $\mathcal{F}(\bar{A}) / \operatorname{ker} \Psi_{\theta}$, thus, $A_{\theta}$ is a Banach space.

Let us show that the space $A_{\theta}$ is in fact an interpolation space according to the Definition 2.2.5.

Theorem 2.3.5. $A_{\theta}$ is an interpolation space with respect to $\left(A_{0}, A_{1}\right)$.

Proof. Let us first show that $A_{\theta}$ is an intermediate space between $A_{0}$ and $A_{1}$, i.e. $A_{0} \cap A_{1} \hookrightarrow A_{\theta} \hookrightarrow$ $A_{0}+A_{1}$.

Let $a \in A_{\theta}$ and $\epsilon>0$. By the infimum property there is $f \in \mathcal{F}(\bar{A})$ such that $f(\theta)=a \in A_{0}+A_{1}$ and $\|f\|_{\mathcal{F}(\bar{A})} \leq\|a\|_{A_{\theta}}+\epsilon$. Thus, $\|a\|_{A_{0}+A_{1}}=\|f(\theta)\|_{A_{0}+A_{1}} \leq\|f\|_{\mathcal{F}(\bar{A})} \leq\|a\|_{A_{\theta}}+\epsilon$, from which follows that 
$\|a\|_{A_{0}+A_{1}} \leq\|a\|_{A_{\theta}}$, and $A_{\theta} \hookrightarrow A_{0}+A_{1}$.

Now, consider $a \in A_{0} \cap A_{1}, \delta>0$ and $f_{\delta}: \overline{\mathbb{S}} \longrightarrow A_{0}+A_{1}$ given by $f_{\delta}(z)=e^{\delta(z-\theta)^{2}} a$. Note that for every $a^{*} \in\left(A_{0}+A_{1}\right)^{*}$ we have that $a^{*}\left(f_{\delta}(z)\right)=e^{\delta(z-\theta)^{2}} a^{*}(a)$ is holomorphic on $\mathbb{S}$. Moreover, if $\left(z_{n}\right)$ is a sequence in $\overline{\mathbb{S}}$ such that $z_{n} \rightarrow z \in \overline{\mathbb{S}}$, we have

$$
\left\|f_{\delta}\left(z_{n}\right)-f_{\delta}(z)\right\|_{A_{0}+A_{1}}=\left\|e^{\delta\left(z_{n}-\theta\right)^{2}} a-e^{\delta(z-\theta)^{2}} a\right\|_{A_{0}+A_{1}}=\left|e^{\delta\left(z_{n}-\theta\right)^{2}}-e^{\delta(z-\theta)^{2}}\right|\|a\|_{A_{0}+A_{1}} \stackrel{n \rightarrow \infty}{\longrightarrow} 0 .
$$

Note that $e^{\delta(z-\theta)^{2}}$ is bounded on $\overline{\mathbb{S}}$, and thus $f_{\delta}$ is bounded on $\overline{\mathbb{S}}$. In addition, for every $t \in \mathbb{R}$ and $j=0,1$ we have

$$
A_{j} \ni f_{\delta}(j+i t)=e^{\delta(j+i t)^{2}} a=e^{\delta\left(j^{2}+2 j i t-t^{2}\right)} a
$$

and thus $t \longmapsto f_{\delta}(j+i t)$ is continuous from $\mathbb{R}$ to $A_{j}$, and

$$
\|f(j+i t)\|_{A_{j}}=e^{\delta j^{2}} e^{-\delta t^{2}}\|a\|_{A_{j}} \stackrel{|t| \rightarrow \infty}{\longrightarrow} 0 .
$$

Therefore, $f \in \mathcal{F}(\bar{A})$ and $f(\theta)=a \in A_{\theta}$. Lastly, note that

$$
\|a\|_{A_{\theta}} \leq\left\|f_{\delta}\right\|_{\mathcal{F}(\bar{A})}=\max _{j=0,1}\left\{\sup _{t \in \mathbb{R}}\left\|f_{\delta}(j+i t)\right\|_{A_{j}}\right\}=\max _{j=0,1}\left\{\sup _{t \in \mathbb{R}} e^{\delta j-\delta t^{2}}\|a\|_{A_{j}}\right\} \leq e^{\delta}\|a\|_{A_{0} \cap A_{1}} .
$$

Taking the limit $\delta \longrightarrow 0^{+}$in the previous equation, we have that $\|a\|_{A_{\theta}} \leq\|a\|_{A_{0} \cap A_{1}}$.

Thus, $A_{0} \cap A_{1} \hookrightarrow A_{\theta}$, and $A_{\theta}$ is an intermediate space between $A_{0}$ and $A_{1}$.

Let $T \in \mathcal{B}\left(A_{0}+A_{1}\right)$ be such that $T_{A_{j}} \in \mathcal{B}\left(A_{j}\right), j=0,1$. Let us show that $T_{A_{\theta}} \in \mathcal{B}\left(A_{\theta}\right)$. Consider $M_{j}=\|T\|_{\mathcal{B}\left(A_{j}\right)}, j=0,1$. Let $a \in A_{\theta}, \epsilon>0$, and $f \in \mathcal{F}(\bar{A})$ be such that $f(\theta)=a$ and $\|f\|_{\mathcal{F}(\bar{A})} \leq\|a\|_{A_{\theta}}+\epsilon$. Consider $g: \overline{\mathbb{S}} \longrightarrow A_{0}+A_{1}$ given by

$$
g(z)=M_{0}^{z-1} M_{1}^{-z} T(f(z))
$$

Note that $g$ is continuous. Moreover, for $z=r+i t \in \overline{\mathbb{S}}$ we have

$$
\begin{aligned}
\|g(z)\|_{A_{0}+A_{1}} & =\left|M^{r+i t-1} M_{1}^{-r-i t}\right|\|T(f(z))\|_{A_{0}+A_{1}}=M_{0}^{r-1} M_{1}^{-r}\|T(f(z))\|_{A_{0}+A_{1}} \\
& \leq M_{0}^{r-1} M_{1}^{-r}\|T\|_{\mathcal{B}\left(A_{0}+A_{1}\right)}\|f(z)\|_{A_{0}+A_{1}}
\end{aligned}
$$

and since $0 \leq r \leq 1$ and $f$ is bounded on $\overline{\mathbb{S}}$, it follows that $g$ is bounded on $\overline{\mathbb{S}}$. Moreover, for $a^{*} \in\left(A_{0}+A_{1}\right)^{*}$ we have that $a^{*} \circ T \in\left(A_{0}+A_{1}\right)^{*}$. Since $f: \overline{\mathbb{S}} \longrightarrow A_{0}+A_{1}$ is holomorphic on $\mathbb{S}$, we have that

$$
a^{*}(g(z))=a^{*}\left(M_{0}^{z-1} M_{1}^{z}(T(f(z)))=M_{0}^{z-1} M_{1}^{z} a^{*}(T(f(z)))\right.
$$

is holomorphic on $\mathbb{S}$. Thus, $g$ is holomorphic on $\mathbb{S}$. Note also that for $t \in \mathbb{R}$ and $j=0,1$

$$
g(j+i t)=M_{0}^{j+i t-1} M_{1}^{-j-i t} T(f(j+i t)) \in A_{j}
$$


thus, $t \longmapsto g(j+i t) \in A_{j}$ is continuous, $j=0,1$. Furthermore,

$$
\begin{aligned}
\|g(j+i t)\|_{A_{j}} & =\left|M_{0}^{j+i t-1} M_{1}^{-j-i t}\right|\|T(f(j+i t))\|_{A_{j}}=M_{0}^{j-1} M_{1}^{-j}\|T(f(j+i t))\|_{A_{j}} \\
& \leq M_{0}^{j-1} M_{1}^{-j}\|T\|_{\mathcal{B}\left(A_{j}\right)}\|f(j+i t)\|_{A_{j}}=M_{0}^{j-1} M_{1}^{-j} M_{j}\|f(j+i t)\|_{A_{j}} \\
& =\|f(j+i t)\|_{A_{j}} \stackrel{|t| \rightarrow \infty}{\longrightarrow} 0
\end{aligned}
$$

Thus, $g \in \mathcal{F}(\bar{A})$ and $A_{\theta} \ni g(\theta)=M_{0}^{\theta-1} M_{1}^{-\theta} T(a)$, and $T(a) \in A_{\theta}$.

Finally, let us show that $T_{A_{\theta}} \in \mathcal{B}\left(A_{\theta}\right)$. It follows from (2.9) that

$$
\|g\|_{\mathcal{F}(\bar{A})}=\max _{j=0,1}\left\{\sup _{t \in \mathbb{R}}\|g(j+i t)\|_{A_{j}}\right\} \leq \max _{j=0,1}\left\{\sup _{t \in \mathbb{R}}\|f(j+i t)\|_{A_{j}}\right\}=\|f\|_{\mathcal{F}(\bar{A})} .
$$

Thus,

$$
\begin{aligned}
\|T(a)\|_{A_{\theta}} & =\left\|g(\theta) M_{0}^{1-\theta} M_{1}^{\theta}\right\|_{A_{\theta}}=M_{0}^{1-\theta} M_{1}^{\theta}\|g(\theta)\|_{A_{\theta}} \\
& \leq M_{0}^{1-\theta} M_{1}^{\theta}\|g\|_{\mathcal{F}(\bar{A})} \leq M_{0}^{1-\theta} M_{1}^{\theta}\|f\|_{\mathcal{F}(\bar{A})} \leq M_{0}^{1-\theta} M_{1}^{\theta}\left(\|a\|_{A_{\theta}}+\epsilon\right)
\end{aligned}
$$

Since $\epsilon>0$ is arbitrary, it follows that $T_{A_{\theta}} \in \mathcal{B}\left(A_{\theta}\right)$.

Next, we present two results that will be useful later on.

Theorem 2.3.6. Let $\left(A_{0}, A_{1}\right)$ be a compatible Banach couple and $0<\theta<1$. Then $A_{0} \cap A_{1}$ is dense in $A_{\theta}$.

Proof. See [BL12], Theorem 4.2.2.

Theorem 2.3.7. Let $\left(A_{0}, A_{1}\right)$ be a compatible Banach couple and $0<\theta<1$. Suppose that $A_{0} \cap A_{1}$ is dense in both $A_{0}$ and $A_{1}$ and that at least one of the spaces $A_{0}$ and $A_{1}$ is reflexive. Then

$$
A_{\theta}^{*}=\left(A_{0}, A_{1}\right)_{\theta}^{*}=\left(A_{0}^{*}, A_{1}^{*}\right)_{\theta},
$$

with equal norms.

Proof. See [BL12], Section 4.5.

Theorem 2.3.8. (The Reiteration Theorem) Let $\left(A_{0}, A_{1}\right)$ be a compatible Banach couple and $0 \leq \theta_{0}, \theta_{1} \leq 1$. Assume that $A_{0} \cap A_{1}$ is dense in $A_{0}, A_{1}$ and $A_{\theta_{0}} \cap A_{\theta_{1}}$. Then, for every $0 \leq \gamma \leq 1$,

$$
\left(A_{\theta_{0}}, A_{\theta_{1}}\right)_{\gamma}=\left(A_{0}, A_{1}\right)_{\theta}, \quad \theta=(1-\gamma) \theta_{0}+\gamma \theta_{1},
$$

with equal norms.

Proof. See [BL12], Theorem 4.6.1. 


\subsection{Complex Interpolation of $L_{p}$ spaces}

Let $(\Omega, \mathcal{M}, \mu)$ be a measure space and for $1 \leq p \leq \infty$, put $L_{p}=L_{p}(\Omega, \mathcal{M}, \mu)$. We saw in Example 2.2.6 that if $1 \leq p_{0}<p<p_{1} \leq \infty$ then $L_{p}$ is an interpolated space between $L_{p_{0}}$ and $L_{p_{1}}$. In order to illustrate the complex interpolation method, we will consider the complex interpolation of $L_{p}$ spaces and verify that we obtain the same result. That is, we will prove the following result:

Theorem 2.4.1. Let $1<p_{0}<p_{1}<\infty$ and $0<\theta<1$. Then

$$
\left(L_{p_{0}}, L_{p_{1}}\right)_{\theta}=L_{p}, \quad \frac{1}{p}=\frac{1-\theta}{p_{0}}+\frac{\theta}{p_{1}}
$$

with equal norms.

Put $A_{0}=L_{p_{0}}, A_{1}=L_{p_{1}}, A_{\theta}=\left(L_{p_{0}}, L_{p_{1}}\right)_{\theta}$ and $\bar{A}=\left(L_{p_{0}}, L_{p_{1}}\right)$. We will use the following Lemma:

Lemma 2.4.2. Let

$$
\mathcal{D}=\left\{a=\sum_{n=1}^{m} \alpha_{n} \chi_{E_{n}}: a \text { is a simple function, } m \in \mathbb{N}, \mu\left(E_{n}\right)<\infty, n=1, \ldots, m\right\} .
$$

Then $\mathcal{D}$ is dense in both $L_{p}$ and $A_{\theta}$.

Proof. We already know from Proposition 1.2.6 that $\mathcal{D}$ is dense in $L_{p}, L_{p_{0}}$ and $L_{p_{1}}$. Thus, $\mathcal{D}$ is dense in $L_{p_{0}} \cap L_{p_{1}}$. It follows from Theorem 2.3.6 and the fact that $L_{p_{0}} \cap L_{p_{1}} \rightarrow A_{\theta}$ that $\mathcal{D}$ is dense in $A_{\theta}$.

Proof of Theorem 2.4.1: To begin, let us show that it is sufficient to prove that

$$
\|a\|_{A_{\theta}}=\|a\|_{L_{p}}, \forall a \in \mathcal{D} \text {. }
$$

Let $a \in A_{\theta}$. From Lemma 2.4.2, there is a sequence $\left(a_{n}\right) \subset \mathcal{D}$ such that $\left\|a-a_{n}\right\|_{A_{\theta}} \stackrel{n \rightarrow \infty}{\longrightarrow} 0$. Assuming (2.10) is valid, and since $\left(a_{n}\right)$ is a Cauchy sequence in $A_{\theta}$, it follows that $\left(a_{n}\right)$ is a Cauchy sequence in $L_{p}$. Thus, there is $b \in L_{p}$ such that $\left\|b-a_{n}\right\|_{L_{p}} \stackrel{n \rightarrow \infty}{\longrightarrow} 0$.

From Example 2.2.6, we have

$$
\|c\|_{L_{p_{0}}+L_{p_{1}}} \leq 2\|c\|_{L_{p}}, \forall c \in L_{p}
$$

Since $A_{\theta}$ is an intermediate space between $\left(A_{0}, A_{1}\right)$, we have $A_{\theta} \hookrightarrow A_{0}+A_{1}$. Thus, there is $C \geq 0$ such that $\|d\|_{A_{0}+A_{1}} \leq C\|d\|_{A_{\theta}}, \forall c \in A_{\theta}$. Therefore,

$$
\|a-b\|_{A_{0}+A_{1}} \leq\left\|a-a_{n}\right\|_{A_{0}+A_{1}}+\left\|b-a_{n}\right\|_{A_{0}+A_{1}} \leq C\left\|a-a_{n}\right\|_{A_{\theta}}+2\left\|b-a_{n}\right\|_{L_{p}} \stackrel{n \rightarrow \infty}{\longrightarrow} 0
$$

Hence, $a=b \in A_{\theta} \subseteq L_{p}$. Similarly, it is shown that $L_{p} \subseteq A_{\theta}$. Moreover,

$$
\|a\|_{A_{\theta}}=\lim _{n \rightarrow \infty}\left\|a_{n}\right\|_{A_{\theta}}=\lim _{n \rightarrow \infty}\left\|a_{n}\right\|_{L_{p}}=\|a\|_{L_{p}}
$$


we therefore have that (2.10) implies the desired result.

Consider, then, $a \in \mathcal{D}$. Let us show that $\|a\|_{A_{\theta}}=\|a\|_{L_{p}}$. Let us write $a=\sum_{n=1}^{m} \alpha_{n} \chi_{E_{n}}$ in the canonical form, i.e. $\alpha_{n} \neq 0, E_{i} \cap E_{j}=\varnothing$ if $i \neq j, \mu\left(E_{n}\right)<\infty$. Suppose initially that $\|a\|_{L_{p}}=1$.

For each $z \in \overline{\mathbb{S}}$, define

$$
f_{\epsilon}(z)=e^{\epsilon\left(z^{2}-\theta^{2}\right)}|a|^{\frac{p}{p(z)}} \frac{a}{|a|},
$$

where $\frac{1}{p(z)}=\frac{1-z}{p_{0}}+\frac{z}{p_{1}}$ and $\epsilon>0$. Let us show that $f_{\epsilon} \in \mathcal{F}(\bar{A})$ :

(i) $f_{\epsilon}: \overline{\mathbb{S}} \longrightarrow L_{p_{0}}+L_{p_{1}}$ is well-defined: For each $z=r+i t \in \overline{\mathbb{S}}$ we have

$$
\left|f_{\epsilon}(z)\right|=\left.\left.\left|e^{\epsilon\left((r+i t)^{2}-\theta^{2}\right)}\right||| a\right|^{\frac{p}{p(r+i t)}}|\frac{|a|}{|a|}=\underbrace{e^{\epsilon r^{2}}}_{\leq e^{\epsilon}} \underbrace{e^{-\epsilon t^{2}}}_{\leq 1} \underbrace{e^{-\epsilon \theta^{2}}}_{\leq 1}| a\right|^{\frac{p}{p(r)}} \leq e^{\epsilon}|a|^{\frac{p}{p(r)}}
$$

Since $p_{0} \leq p(r) \leq p_{1}$, we have

$$
\int\left|f_{\epsilon}(z)\right|^{p(r)} \leq \int e^{\epsilon p(r)}|a|^{\frac{p}{p(r)} p(r)} \leq e^{\epsilon p_{1}} \int|a|^{p}=e^{\epsilon p_{1}}
$$

Thus, $f_{\epsilon}(z) \in L_{p(r)} \subseteq L_{p_{0}}+L_{p_{1}}$.

(ii) $f_{\epsilon}$ is holomorphic on $\mathbb{S}$ : Let $\varphi \in\left(L_{p_{0}}+L_{p_{1}}\right)^{*}$. We have, for $z \in \mathbb{S}$,

$$
\begin{aligned}
\varphi\left(f_{\epsilon}(z)\right) & =e^{\epsilon\left(z^{2}-\theta^{2}\right)} \varphi\left(\left|\sum_{n=1}^{m} \alpha_{n} \chi_{E_{n}}\right| \frac{p}{p(z)} \frac{a}{|a|}\right) \\
& =e^{\epsilon\left(z^{2}-\theta^{2}\right)} \varphi\left(\sum_{n=1}^{m}\left|\alpha_{n}\right|^{\frac{p}{p(z)}} \frac{a}{|a|^{2}} \chi_{E_{n}}\right) \\
& =e^{\epsilon\left(z^{2}-\theta^{2}\right)}\left[\sum_{n=1}^{m}\left|\alpha_{n}\right|^{\frac{p}{p(z)}} \varphi\left(\frac{a}{|a|} \chi_{E_{n}}\right)\right]
\end{aligned}
$$

Note that $\varphi \circ f_{\epsilon}$ is holomorphic in the usual (scalar) sense, thus, $f_{\epsilon}$ is holomorphic.

(iii) $f_{\epsilon}$ is bounded over $\overline{\mathbb{S}}$ : For all $z \in \overline{\mathbb{S}}$, it follows from (2.11) and (2.12) that

$$
\left\|f_{\epsilon}(z)\right\|_{L_{p_{0}}+L_{p_{1}}} \leq 2\left\|f_{\epsilon}(z)\right\|_{L_{p(r)}} \leq\left(e^{\epsilon p_{1}}\right)^{\frac{1}{p(r)}} \leq e^{\epsilon \frac{p_{1}}{p_{0}}}
$$

(iv) $f_{\epsilon}$ is continuous over $\overline{\mathbb{S}}$ : Indeed, it is immediate that $f_{\epsilon}(z)=\sum_{n=1}^{m} e^{\epsilon\left(z^{2}-\theta^{2}\right)}\left|\alpha_{n}\right|^{\frac{p}{p(z)}} \frac{a}{|a|} \chi_{E_{n}}$ is continuous.

(v) Note finally that $f_{\epsilon}(j+i t) \in L_{p(j)}=L_{p_{j}}, j=0,1, t \in \mathbb{R}$. Moreover,

$$
f_{\epsilon}(j+i t)=\sum_{n=1}^{m} e^{\epsilon\left((j+i t)^{2}-\theta^{2}\right)}\left|\alpha_{n}\right|^{\frac{p}{p(z)}} \frac{a}{|a|} \chi_{E_{n}}
$$

and therefore, $t \mapsto f_{\epsilon}(j+i t)$ is continuous from $\mathbb{R}$ to $L_{p_{j}}$. Furthermore,

$$
\left\|f_{\epsilon}(j+i t)\right\|_{A_{j}}^{p_{j}}=\int\left|f_{\epsilon}(j+i t)\right|^{p_{j}} \leq e^{\epsilon p_{j}} e^{-\epsilon t^{2} p_{j}} \int|a|^{p}=e^{\epsilon p_{j}} e^{-\epsilon t^{2} p_{j}} \stackrel{|t| \rightarrow \infty}{\longrightarrow} 0
$$


Hence, $f_{\epsilon} \in \mathcal{F}(\bar{A})$. Note that

$$
\left\|f_{\epsilon}\right\|_{\mathcal{F}(\bar{A})}=\max \left\{\sup _{t \in \mathbb{R}}\left\|f_{\epsilon}(i t)\right\|_{A_{0}}, \sup _{t \in \mathbb{R}}\left\|f_{\epsilon}(1+i t)\right\|_{A_{1}}\right\} \leq e^{\epsilon}
$$

Thus, since $f_{\epsilon}(\theta)=a$, we have

$$
\|a\|_{A_{\theta}}=\inf \left\{\|g\|_{\mathcal{F}(\bar{A})}: g \in \mathcal{F}(\bar{A}), g(\theta)=a\right\} \leq\left\|f_{\epsilon}\right\|_{\mathcal{F}(\bar{A})} \leq e^{\epsilon}
$$

Taking the limit $\epsilon \longrightarrow 0^{+}$in the previous equation, we have that $\|a\|_{A_{\theta}} \leq 1$.

If $\|a\|_{L_{p}} \neq 1$, we can use the homogeneity of the norm to prove that $\|a\|_{A_{\theta}} \leq\|a\|_{L_{p}}$.

For the other inequality consider $a \in \mathcal{D}$. If $p^{\prime}$ denotes the conjugate exponent of $p$, we have from Theorem 1.2.9 that

$$
\|a\|_{L_{p}}=\sup \left\{\left|\int a b\right|: b \in \mathcal{D},\|b\|_{L_{p^{\prime}}}=1\right\}
$$

Let $\epsilon>0$. From the infimum property and the definition of the norm in $A_{\theta}$, there is $f \in \mathcal{F}(\bar{A})$ such that $f(\theta)=a$ and $\|f\|_{\mathcal{F}(\bar{A})} \leq\|a\|_{A_{\theta}}+\epsilon$. Let $b \in \mathcal{D}$ be such that $\|b\|_{L_{p^{\prime}}}=1$. For each $z \in \overline{\mathbb{S}}$ we define

$$
\begin{gathered}
g(z)=|b|^{\frac{p^{\prime}}{p^{\prime}(z)}} \frac{b}{|b|}, \quad \text { where } \frac{1}{p^{\prime}(z)}=\frac{1-z}{p_{0}^{\prime}}+\frac{z}{p_{1}^{\prime}} \\
F(z)=\int f(z) g(z)
\end{gathered}
$$

It follows from the Lebesgue's Dominated Convergence Theorem (Theorem 1.2.1) that $F: \mathbb{S} \longrightarrow \mathbb{C}$ is continuous. Writing $b=\sum_{n=1}^{m} \beta_{n} \chi_{F_{n}}$, with $\beta_{n} \neq 0$ and $F_{i} \cap F_{j}=\varnothing$, if $j \neq i$, we have, for $z=r+i t \in \overline{\mathbb{S}}$,

$$
\begin{aligned}
|F(z)| & =\left|\int f(z) g(z)\right| \leq \int|f(z) g(z)|=\int|f(z)||b|^{\frac{p^{\prime}}{p^{\prime}(r)}}=\left.\int|f(z)|\left|\sum_{n=1}^{m} \beta_{n} \chi_{F_{n}}\right|\right|^{\frac{p^{\prime}}{p^{\prime}(r)}} \\
& =\sum_{n=1}^{m} \int_{F_{n}}|f(z) \| \beta|^{\frac{p^{\prime}}{p^{\prime}(r)}}=\sum_{n=1}^{m}|\beta|^{\frac{p^{\prime}}{p^{\prime}(r)}} \int_{F_{n}}|f(z)|
\end{aligned}
$$

Note that, since $f(z) \in L_{p_{0}}+L_{p_{1}} \subseteq L_{1}$ and $0 \leq r \leq 1$ (thus $|\beta|^{\frac{p^{\prime}}{p^{\prime}(r)}}$ is bounded), it follows that $|F(z)|$ is bounded on $\overline{\mathbb{S}}$.

Let $\gamma$ be a closed piecewise $C^{1}$ curve in $\mathbb{S}$. We have

$$
\begin{aligned}
\int_{\gamma} F(z) d z & =\int_{\gamma}\left[\int_{\Omega} f(z)(s) g(z)(s) d \mu(s)\right] d z \\
& =\int_{\gamma}\left[\int_{\Omega} f(z)(s)\left(\frac{b(s)}{|b(s)|} \sum_{n=1}^{m}\left|\beta_{n}\right|^{\frac{p^{\prime}}{p^{\prime}(z)}} \chi_{F_{n}}(s)\right) d \mu(s)\right] d z \\
& =\sum_{n=1}^{m} \int_{F_{n}} \frac{b(s)}{|b(s)|}\left[\int_{\gamma} f(z)(s)\left|\beta_{n}\right|^{\frac{p^{\prime}}{p^{\prime}(z)}} d z\right] d \mu(s)
\end{aligned}
$$

Since for each fixed $s \in \Omega$, we have that

$$
z \longmapsto f(z)(s)\left|\beta_{n}\right|^{\frac{p^{\prime}}{p^{\prime}(z)}}
$$


is holomorphic on $\mathbb{S}$, we have that from Cauchy's Integral Theorem (Theorem 1.4.2) that

$$
\int_{\gamma} f(z)(s)\left|\beta_{n}\right|^{\frac{p^{\prime}}{p^{\prime}(z)}} d z=0
$$

Thus, $\int_{\gamma} F(z) d z=0$, and from Morera's Theorem (Theorem 1.4.3), it follows that $F$ is holomorphic on $\mathbb{S}$.

Note that, for all $t \in \mathbb{R}$ and $j=0,1$,

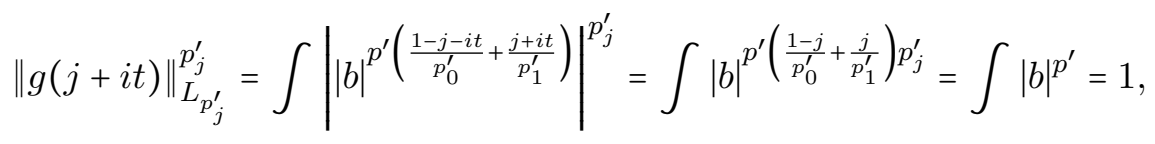

thus, from Hölder's Inequality (Theorem 1.2.2),

$$
|F(j+i t)| \leq \int|f(j+i t) g(j+i t)| \leq\|f(j+i t)\|_{L_{p_{j}}}\|g(j+i t)\|_{L_{p_{j}^{\prime}}}=\|f(j+i t)\|_{L_{p_{j}}}
$$

Now, by the Maximum Principle of Phragmén-Lindelöf (Theorem 1.4.6), we have for all $z \in \mathbb{S}$

$$
|F(z)| \leq \max _{j=0,1}\left\{\sup _{t \in \mathbb{R}}|F(j+i t)|\right\} \leq \max _{j=0,1}\left\{\sup _{t \in \mathbb{R}}\|f(j+i t)\|_{L_{p_{j}}}\right\}=\|f\|_{\mathcal{F}(\bar{A})}
$$

So, note that

$$
|F(\theta)|=\left|\int f(\theta) g(\theta)\right|=\left|\int a b\right| \leq\|f\|_{\mathcal{F}(\bar{A})}
$$

Thus,

$$
\|a\|_{L_{p}}=\sup \left\{\left|\int a b\right|: b \in \mathcal{D},\|b\|_{L_{p^{\prime}}}=1\right\} \leq\|f\|_{\mathcal{F}(\bar{A})} \leq\|a\|_{A_{\theta}}+\epsilon
$$

Since $\epsilon>0$ is arbitrary, we have that $\|a\|_{L_{p}} \leq\|a\|_{A_{\theta}}$.

\subsection{Complex Interpolation of Weighted $L_{p}$ spaces}

In this section, in order to illustrate the complex interpolation method, we will find the weighted $L_{p}$ interpolation scale in the case of $L_{1}$-weights. The proof presented here is very similar to that of the previous section, we consider only a few changes in the arguments.

Consider $\Omega=(0,1), \mathcal{M}$ the Borel $\sigma$-algebra on $(0,1)$ and $m$ the Lebesgue measure. Let $w_{0}, w_{1}$ : $(0,1) \longrightarrow(0, \infty)$ such that $w_{0}, w_{1} \in L_{1}(\Omega, \mathcal{M}, \mu)$. We have the following result:

Theorem 2.5.1. For $1<p<\infty$ and $0<\theta<1$ we have, with equal norms,

$$
\left(L_{p}\left(w_{0}\right), L_{p}\left(w_{1}\right)\right)_{\theta}=L_{p}(w)
$$

where $w=w_{0}^{1-\theta} w_{1}^{\theta}$. 
Proof. Consider $A_{0}=L_{p}\left(w_{0}\right), A_{1}=L_{p}\left(w_{1}\right), A_{\theta}=\left(L_{p}\left(w_{0}\right), L_{p}\left(w_{1}\right)\right)_{\theta}$ and $\bar{A}=\left(L_{p}\left(w_{0}\right), L_{p}\left(w_{1}\right)\right)$. Denote $\nu_{0}=w_{0} d m, \nu_{1}=w_{1} d m$ and $\nu=w d m$. First, let us see that it is enough to show that

$$
\|a\|_{A_{\theta}}=\|a\|_{L_{p}(w)}, \forall a \in \mathcal{D}
$$

where $\mathcal{D}=\left\{a=\sum_{i=1}^{n} \alpha_{i} \chi_{E_{i}}: a\right.$ is a simple function, $\left.n \in \mathbb{N}, \nu\left(E_{i}\right)<\infty, i=1, \ldots, n\right\}$.

If

$$
\mathcal{D}_{j}=\left\{a=\sum_{i=1}^{n} \alpha_{i} \chi_{E_{i}}: a \text { is a simple function, } n \in \mathbb{N}, \nu_{j}\left(E_{i}\right)<\infty, i=1, \ldots, n\right\}, j=0,1
$$

note that, since $w_{0}, w_{1} \in L_{1}$ and $w_{0}^{1-\theta} w_{1}^{\theta} \leq(1-\theta) w_{0}+\theta w_{1}$, we have $\mathcal{D}_{j}=\mathcal{D}$, from which follows that $\mathcal{D}$ dense in both $L_{p}(w)$ and $A_{\theta}$ (see Lemma 2.4.2).

Let $a \in A_{\theta}$. Then there is a sequence $\left(a_{n}\right)$ in $\mathcal{D}$ such that $\left\|a_{n}-a\right\|_{A_{\theta}} \stackrel{n \rightarrow \infty}{\longrightarrow} 0$. Assuming that (2.13) is valid, we have

$$
\left\|a_{n}-a_{m}\right\|_{A_{\theta}}=\left\|a_{n}-a_{m}\right\|_{L_{p}(w)}, \forall n, m \in \mathbb{N} .
$$

Therefore, $\left(a_{n}\right)$ is a Cauchy sequence in $L_{p}(w)$, and there is $b \in L_{p}(w)$ such that $\left\|a_{n}-a\right\|_{L_{p}(w)} \stackrel{n \rightarrow \infty}{\longrightarrow}$ 0 .

Consider

$$
E=\left\{s \in(0,1): \frac{w_{0}(s)}{w_{1}(s)} \leq 1\right\}
$$

Thus, for $s \in E^{c}$ we have $w_{0}^{1-\theta}(s) w_{1}^{\theta}(s) \geq w_{0}^{\theta}(s) w_{1}^{1-\theta}(s)$, which implies that for each $c \in L_{p}(w)$

$$
\begin{gathered}
\int_{E}|c|^{p} w_{0} d m \leq \int_{E}|c|^{p} w_{0}\left(\frac{w_{1}}{w_{0}}\right)^{\theta} d m=\int_{E}|c|^{p} w d m \\
\int_{E^{c}}|c|^{p} w_{1} d m \leq \int_{E^{c}}|c|^{p} w_{1}\left(\frac{w_{0}}{w_{1}}\right)^{\theta} d m=\int_{E^{c}}|c|^{p} w_{0}^{\theta} w_{1}^{1-\theta} d m \leq \int_{E^{c}}|c|^{p} w d m
\end{gathered}
$$

Thus,

$$
\|c\|_{L_{p}\left(w_{0}\right)+L_{p}\left(w_{1}\right)} \leq\left\|c \chi_{E}\right\|_{L_{p}\left(w_{0}\right)}+\left\|c \chi_{E^{c}}\right\|_{L_{p}\left(w_{1}\right)} \leq\|c\|_{L_{p}(w)}+\|c\|_{L_{p}(w)}=2\|c\|_{L_{p}(w)}, \quad \forall c \in L_{p}(w)
$$

Moreover, since $A_{\theta}$ is an intermediate space with respect to $\left(A_{0}, A_{1}\right)$, and thus $A_{\theta} \hookrightarrow A_{0}+A_{1}$, there is $C \geq 0$ such that

$$
\|d\|_{A_{0}+A_{1}} \leq C\|d\|_{A_{\theta}}, \quad \forall d \in A_{\theta}
$$

Therefore,

$$
\begin{aligned}
\|a-b\|_{L_{p}\left(w_{0}\right)+L_{p}\left(w_{1}\right)} & \leq\left\|a-a_{n}\right\|_{L_{p}\left(w_{0}\right)+L_{p}\left(w_{1}\right)}+\left\|b-a_{n}\right\|_{L_{p}\left(w_{0}\right)+L_{p}\left(w_{1}\right)} \\
& \leq C\left\|a-a_{n}\right\|_{A_{\theta}}+2\left\|b-a_{n}\right\|_{L_{p}(w)} \stackrel{n \rightarrow \infty}{\longrightarrow} 0
\end{aligned}
$$

Hence, $a=b \in A_{\theta} \subseteq L_{p}(w)$. Similarly, it is shown that $L_{p}(w) \subseteq A_{\theta}$. Moreover,

$$
\|a\|_{A_{\theta}}=\lim _{n \rightarrow \infty}\left\|a_{n}\right\|_{A_{\theta}}=\lim _{n \rightarrow \infty}\left\|a_{n}\right\|_{L_{p}(w)}=\|a\|_{L_{p}(w)}
$$


we therefore have that (2.13) implies the desired result.

Theus, let $a \in \mathcal{D}$ and suppose initially that $\|a\|_{L_{p}(w)}=1$. Consider, for each $z \in \overline{\mathbb{S}}$ and $s \in(0,1)$,

$$
f_{\epsilon}(z)(s)=e^{\epsilon\left(z^{2}-\theta^{2}\right)} w_{0}^{\frac{z-\theta}{p}}(s) w_{1}^{\frac{\theta-z}{p}}(s) a(s)
$$

where $\epsilon>0$. Let us show that $f_{\epsilon} \in \mathcal{F}(\bar{A})$ :

(i) $f_{\epsilon}: \overline{\mathbb{S}} \longrightarrow L_{p}\left(w_{0}\right)+L_{p}\left(w_{1}\right)$ is well-defined: Given $z=r+i t \in \overline{\mathbb{S}}$, we have

$$
\left|f_{\epsilon}(z)\right|=e^{\epsilon r^{2}} e^{-\epsilon t^{2}} e^{-\epsilon \theta^{2}} w_{0}^{\frac{r-\theta}{p}} w_{1}^{\frac{\theta-r}{p}}|a| \leq e^{\epsilon} w_{0}^{\frac{r-\theta}{p}} w_{1}^{\frac{\theta-r}{p}}|a|
$$

Thus,

$\int\left|f_{\epsilon}(z)\right|^{p} w_{0}^{1-r} w_{1}^{r} d m \leq e^{\epsilon p} \int|a|^{p} w_{0}^{r-\theta} w_{1}^{\theta-r} w_{0}^{1-r} w_{1}^{r} d m=e^{\epsilon p} \int|a|^{p} w^{1-\theta} w_{1}^{\theta} d m=e^{\epsilon p}\|a\|_{L_{p}(w)}^{p}=e^{\epsilon p}$ and we have that $f_{\epsilon}(r+i t) \in L_{p}\left(w_{0}^{1-r} w_{1}^{r}\right)$. Moreover, considering $E$ as in (2.14), we have

$$
\begin{aligned}
\int_{E}\left|f_{\epsilon}(z)\right|^{p} w_{0} d m & \leq e^{\epsilon p} \int_{E}|a|^{p} w_{0}^{1-r} w_{1}^{r} w_{0} d m=e^{\epsilon p} \int_{E}|a|^{p} w_{0}^{1-\theta} w_{1}^{\theta}\left(\frac{w_{0}}{w_{1}}\right)^{r} d m \\
& \leq e^{\epsilon p} \int_{E}|a|^{p} w_{0}^{1-\theta} w_{1}^{\theta} d m \leq e^{\epsilon p}\|a\|_{L_{p}(w)}^{p}=e^{\epsilon p} \\
\int_{E^{c}}\left|f_{\epsilon}(z)\right|^{p} w_{1} d m & \leq \int_{E^{c}}\left|f_{\epsilon}(z)\right|^{p} w_{1}\left(\frac{w_{0}}{w_{1}}\right)^{r} d m=\int_{E^{c}}\left|f_{\epsilon}(z)\right|^{p} w_{0}^{r} w_{1}^{1-r} d m \\
& \leq e^{\epsilon p} \int_{E^{c}}|a|^{p} w_{0}^{r-\theta} w_{1}^{\theta-r} w_{0}^{1-r} w_{1}^{r} d m \leq e^{\epsilon p}\|a\|_{L_{p}(w)}^{p}=e^{\epsilon p}
\end{aligned}
$$

Thus, $f_{\epsilon}(z)=f_{\epsilon}(z) \chi_{E}+f_{\epsilon}(z) \chi_{E^{c}} \in L_{p}\left(w_{0}\right)+L_{p}\left(w_{1}\right)$ is well-defined.

(ii) $f_{\epsilon}$ is bounded on $\overline{\mathbb{S}}$ : It follows from $(2.20),(2.21)$ and the definition of the norm in $L_{p}\left(w_{0}\right)+$ $L_{p}\left(w_{1}\right)$ that, for all $z \in \overline{\mathbb{S}}$ :

$$
\left\|f_{\epsilon}(z)\right\|_{L_{p}\left(w_{0}\right)+L_{p}\left(w_{1}\right)} \leq\left\|f_{\epsilon}(z) \chi_{E}\right\|_{L_{p}\left(w_{0}\right)}+\left\|f_{\epsilon}(z) \chi_{E^{c}}\right\|_{L_{p}\left(w_{1}\right)} \leq 2 e^{\epsilon}\|a\|_{L_{p}(w)}
$$

(iii) $f_{\epsilon}$ is continuous on $\overline{\mathbb{S}}$ : Let $z \in \overline{\mathbb{S}}$ and consider a sequence $\left(z_{n}\right)$ such that $z_{n} \rightarrow z$. For each $s \in E$ and $n \in \mathbb{N}$, define

$$
\begin{gathered}
F_{n}(s)=f_{\epsilon}\left(z_{n}\right)(s) \\
F(s)=f_{\epsilon}(z)(s)
\end{gathered}
$$

Note that $F_{n}(s) \rightarrow F(s)$ for every $s \in E$. Moreover, writing $z_{n}=r_{n}+i t_{n}$ for all $n \in \mathbb{N}$, we have

$$
\left|F_{n}(s)\right| \leq w_{0}^{\frac{r_{n}-\theta}{p}}(s) w_{1}^{\frac{\theta-r_{n}}{p}}(s)|a(s)|
$$

It follows from (2.20) that $w_{0}^{\frac{r_{n}-\theta}{p}}(s) w_{1}^{\frac{\theta-r_{n}}{p}}(s)|a(s)| \in L_{p}\left(E,\left.\mathcal{M}\right|_{E},\left.w_{0}\right|_{E}\right)$. Thus, from the Lebesgue's Dominated Convergence Theorem (Theorem 1.2.1), it follows that

$$
\left\|f_{\epsilon}\left(z_{n}\right) \chi_{E}-f_{\epsilon}(z) \chi_{E}\right\|_{L_{p}\left(w_{0}\right)}=\left\|F_{n}-F\right\|_{L_{p}\left(E,\left.\mathcal{M}\right|_{E},\left.w_{0}\right|_{E}\right)} \stackrel{n \rightarrow \infty}{\longrightarrow} 0
$$


Similarly, it is shown that

$$
\left\|f_{\epsilon}\left(z_{n}\right) \chi_{E^{c}}-f_{\epsilon}(z) \chi_{E^{c}}\right\|_{L_{p}\left(w_{1}\right)} \stackrel{n \rightarrow \infty}{\longrightarrow} 0
$$

Therefore,

$\left\|f_{\epsilon}\left(z_{n}\right)-f_{\epsilon}(z)\right\|_{L_{p}\left(w_{0}\right)+L_{p}\left(w_{1}\right)} \leq\left\|f_{\epsilon}\left(z_{n}\right) \chi_{E}-f_{\epsilon}(z) \chi_{E}\right\|_{L_{p}\left(w_{0}\right)}+\left\|f_{\epsilon}\left(z_{n}\right) \chi_{E^{c}}-f_{\epsilon}(z) \chi_{E^{c}}\right\|_{L_{p}\left(w_{1}\right)} \stackrel{n \rightarrow \infty}{\longrightarrow} 0$

from which follows that $f_{\epsilon}$ is continuous on $\overline{\mathbb{S}}$.

(iv) $f_{\epsilon}$ is holomorphic on $\mathbb{S}$ : Let $x^{*} \in\left(L_{p}\left(w_{0}\right)+L_{p}\left(w_{1}\right)\right)^{*}$. From Theorem 2.2.8, we have $\left(L_{p}\left(w_{0}\right)+\right.$ $\left.L_{p}\left(w_{1}\right)\right)^{*}=L_{p}\left(w_{0}\right)^{*} \cap L_{p}\left(w_{1}\right)^{*}$. Since $\nu_{0}=w_{0} d m$ and $\nu_{1}=w_{1} d m$ are positive $\sigma$-finite measures, it follows from an application of the Radon-Nikodým Theorem (Theorem 1.2.5) that there are $g_{0} \in L_{p}\left(w_{0}\right)^{*}$ and $g_{1} \in L_{p}\left(w_{1}\right)^{*}$ such that

$$
x^{*}(h)=\int h g_{j} w_{j} d m, \forall h \in L_{p}\left(w_{j}\right), j=0,1
$$

Thus, we have

$$
\begin{aligned}
x^{*}\left(f_{\epsilon}(z)\right) & =x^{\star}\left(f_{\epsilon}(z) \chi_{E}\right)+x^{*}\left(f_{\epsilon}(z) \chi_{E^{c}}\right)=\int_{E} f_{\epsilon}(z) g_{0} w_{0} d m+\int_{E^{c}} f_{\epsilon}(z) g_{1} w_{1} d m \\
& =\int_{E} e^{\epsilon\left(z^{2}-\theta^{2}\right)} w_{0}^{\frac{z-\theta}{p}} w_{1}^{\frac{\theta-z}{p}} a g_{0} w_{0} d m+\int_{E^{c}} e^{\epsilon\left(z^{2}-\theta^{2}\right)} w_{0}^{\frac{z-\theta}{p}} w_{1}^{\frac{\theta-z}{p}} a g_{1} w_{1} d m
\end{aligned}
$$

Let $\gamma$ be a closed piecewise $C^{1}$ curve in $\mathbb{S}$. We have

$$
\begin{aligned}
\int_{\gamma} x^{*}\left(f_{\epsilon}(z)\right) d z & =\int_{\gamma}\left[\int_{E} e^{\epsilon\left(z^{2}-\theta^{2}\right)} w_{0}^{\frac{z-\theta}{p}}(s) w_{1}^{\frac{\theta-z}{p}}(s) a(s) g_{0}(s) w_{0}(s) d m(s)\right] d z \\
& +\int_{\gamma}\left[\int_{E^{c}} e^{\epsilon\left(z^{2}-\theta^{2}\right)} w_{0}^{\frac{z-\theta}{p}}(s) w_{1}^{\frac{\theta-z}{p}}(s) a(s) g_{1}(s) w_{1}(s) d m(s)\right] d z \\
& =\int_{E} a(s) g_{0}(s) w_{0}(s)\left[\int_{\gamma} e^{\epsilon\left(z^{2}-\theta^{2}\right)} w_{0}^{\frac{z-\theta}{p}}(s) w_{1}^{\frac{\theta-z}{p}}(s) d z\right] d m(s) \\
& +\int_{E^{c}} a(s) g_{1}(s) w_{1}(s)\left[\int_{\gamma} e^{\epsilon\left(z^{2}-\theta^{2}\right)} w_{0}^{\frac{z-\theta}{p}}(s) w_{1}^{\frac{\theta-z}{p}}(s) d z\right] d m(s)
\end{aligned}
$$

Note that for fixed $s \in(0,1)$ we have that

$$
\begin{aligned}
& \overline{\mathbb{S}} \longrightarrow \mathbb{C} \\
& z \longmapsto e^{\epsilon\left(z^{2}-\theta^{2}\right)} w_{0}^{\frac{z-\theta}{p}}(s) w_{1}^{\frac{\theta-z}{p}}(s)
\end{aligned}
$$

is holomorphic on $\mathbb{S}$. It follows from Cauchy's Integral Theorem (Theorem 1.4.2) that for all $s \in(0,1)$,

$$
\int_{\gamma} e^{\epsilon\left(z^{2}-\theta^{2}\right)} w_{0}^{\frac{z-\theta}{p}}(s) w_{1}^{\frac{\theta-z}{p}}(s) d z=0,
$$

therefore, $\int_{\gamma} x^{*}\left(f_{\epsilon}(z)\right) d z=0$. Since $x^{*} \circ f_{\epsilon}$ is continuous on $\overline{\mathbb{S}}$, it follows from Morera's Theorem (Theorem 1.4.3) that $x^{*} \circ f_{\epsilon}$ is holomorphic on $\mathbb{S}$.

(v) Finally, note that $f_{\epsilon}(j+i t) \in L_{p}\left(w_{j}\right)$ for all $t \in \mathbb{R}, j=0,1$ and $t \mapsto f_{\epsilon}(j+i t)$ is continuous. 
Furthermore, from (2.19) we have

$$
\int\left|f_{\epsilon}(j+i t)\right|^{p} w_{j} \leq \int e^{\frac{-\epsilon t^{2}}{p}}|a|^{p} w_{0}^{j-\theta} w_{1}^{\theta-j} w_{j} d m=e^{\frac{-\epsilon t^{2}}{p}} \int|a|^{p} w_{0}^{1-\theta} w_{1}^{\theta} d m=e^{\frac{-\epsilon t^{2}}{p}} \stackrel{|t| \rightarrow \infty}{\longrightarrow} 0
$$

Thus, $\left\|f_{\epsilon}(j+i t)\right\|_{L_{w_{j}}} \stackrel{|t| \rightarrow \infty}{\longrightarrow} 0$, for $j=0,1$.

Therefore, $f_{\epsilon} \in \mathcal{F}(\bar{A})$. Note moreover that $f_{\epsilon}(\theta)=a$ and

$$
\|a\|_{A_{\theta}}=\inf \left\{\|g\|_{\mathcal{F}(\bar{A})}: g \in \mathcal{F}(\bar{A}), g(\theta)=a\right\} \leq\left\|f_{\epsilon}\right\|_{\mathcal{F}(\bar{A})} \leq e^{\epsilon}
$$

Taking the limit $\epsilon \longrightarrow 0^{+}$in the previous equation, we have that $\|a\|_{A_{\theta}} \leq 1=\|a\|_{L_{p}(w)}$. Due to the homogeneity of the norm, it follows that $\|a\|_{A_{\theta}} \leq\|a\|_{L_{p}(w)}$ for all $a \in \mathcal{D}$.

For the other inequality consider $a \in \mathcal{D}$. If $p^{\prime}$ denotes the conjugate exponent of $p$, we have from Theorem 1.2.9 that

$$
\|a\|_{L_{p}(w)}=\sup \left\{\left|\int a b w d m\right|: b \in \mathcal{D},\|b\|_{L_{p^{\prime}}(w)}=1\right\} .
$$

Let $\epsilon>0$. From the infimum property and the definition of the norm in $A_{\theta}$, there is $f \in \mathcal{F}(\bar{A})$ such that $f(\theta)=a$ and $\|f\|_{\mathcal{F}(\bar{A})} \leq\|a\|_{A_{\theta}}+\epsilon$. Let $b \in \mathcal{D}$ be such that $\|b\|_{L_{p^{\prime}}(w)}=1$. For each $z \in \overline{\mathbb{S}}$ define

$$
g(z)=w_{0}^{\frac{z-\theta}{p^{\prime}}} w_{1}^{\frac{\theta-z}{p^{\prime}}}
$$

Note that for $t \in \mathbb{R}$ and $j=0,1$

$$
\int|g(j+i t)|^{p^{\prime}} w_{j} d m=\int|b|^{p^{\prime}} w_{0}^{j-\theta} w_{1}^{\theta-j} w_{j} d m=\int|b|^{p^{\prime}} w_{0}^{1-\theta} w_{1}^{\theta} d m=1
$$

Thus, $\|g(j+i t)\|_{L_{p^{\prime}}\left(w_{j}\right)}=1$. For each $z \in \overline{\mathbb{S}}$, define $F(z)=\int g(z) f(z) w_{0}^{1-z} w_{1}^{z} d m$. From Hölder's inequality (Theorem 1.2.2), it follows that

$$
|F(j+i t)| \leq \int|g(j+i t) f(j+i t)| w_{0}^{1-j} w_{1}^{j} d m \leq\|g(j+i t)\|_{L_{p^{\prime}}\left(w_{j}\right)}\|f(j+i t)\|_{L_{p}\left(w_{j}\right)}=\|f(j+i t)\|_{L_{p}\left(w_{j}\right)}
$$

By the Maximum Principle of Phragmén-Lindelöf (Theorem 1.4.6), we have for all $z \in \mathbb{S}$

$$
|F(z)| \leq \max _{j=0,1}\left\{\sup _{t \in \mathbb{R}}|F(j+i t)|\right\} \leq \max _{j=0,1}\left\{\sup _{t \in \mathbb{R}}\|f(j+i t)\|_{L_{p_{j}}\left(w_{j}\right)}\right\}=\|f\|_{\mathcal{F}(\bar{A})}
$$

Moreover, note that

$$
|F(\theta)|=\left|\int f(\theta) g(\theta)\right| w_{0}^{1-\theta} w_{1}^{\theta}=\left|\int a b w_{0}^{1-\theta} w_{1}^{\theta}\right| \leq\|f\|_{\mathcal{F}(\bar{A})}
$$

Thus,

$$
\|a\|_{L_{p}(w)}=\sup \left\{\left|\int a b w d m\right|: b \in \mathcal{B},\|b\|_{L_{p^{\prime}}(w)}=1\right\} \leq\|f\|_{\mathcal{F}(\bar{A})} \leq\|a\|_{A_{\theta}}+\epsilon
$$

Since $\epsilon>0$ is arbitrary, we have that $\|a\|_{L_{p}(w)} \leq\|a\|_{A_{\theta}}$. 


\subsection{Notes and Comments}

1. The complex interpolation method, initially presented by A. Calderón in [Cal64], can be generalized to families of Banach spaces in various ways which differ by important technical details. See $\left[\mathrm{CCR}^{+} 82\right]$ for a detailed discussion on one of the methods.

2. We will state some other examples of interpolation scales:

2.1. We denote by $C^{\infty}(\mathbb{R})$ the space of all functions on $\mathbb{R}$ whose all derivatives are continuous on $\mathbb{R}$. Let $m \in \mathbb{N}$ and $1 \leq p<\infty$. We put in $C^{\infty}(\mathbb{R})$ the norm

$$
\|f\|_{p, m}=\left(\sum_{n=0}^{m}\left\|f^{(n)}\right\|_{L_{p}}^{m}\right)^{\frac{1}{p}}
$$

where $f^{(n)}$ denotes the nth derivative of $f$. We define the Sobolev Space $W_{p}^{m}(\mathbb{R})$ as the completion of the normed space $\left(C^{\infty}(\mathbb{R}),\|\cdot\|_{p, m}\right)$. For $1<p_{0}, p_{1}<\infty, 0<\theta<1$ and $m \in \mathbb{N}$ we have

$$
\left(W_{p_{0}}^{m}, W_{p_{1}}^{m}\right)_{\theta}=W_{p}^{m}, \quad \frac{1}{p}=\frac{1-\theta}{p_{0}}+\frac{\theta}{p_{1}},
$$

with equivalent norms (see Proposition 8.1 in [Her86]).

2.2. Let $(\Omega, \mathcal{M}, \mu)$ be a measure space. We say that $\varphi:[0, \infty) \longrightarrow[0, \infty)$ is an $N$-function if it is strictly increasing, continuous, $\varphi(0)=0, \lim _{t \rightarrow 0} \frac{\varphi(t)}{t}=0$ and $\lim _{t \rightarrow \infty} \frac{\varphi(t)}{t}=\infty$. An $N$-function $\varphi$ satisfies the $\Delta_{2}$-property if there is $C>0$ such that $\varphi(2 t) \leq C \varphi(t)$ for all $t \geq 0$.

Let $\varphi$ be an $N$-function that satisfies the $\Delta_{2}$-property. We define the Orlicz space $L_{\varphi}(\mu)$ as

$$
L_{\varphi}(\mu)=\left\{f: \Omega \rightarrow \mathbb{C} ; f \text { is measurable, } \varphi(|f|) \in L_{1}(\Omega, \mathcal{M}, \mu)\right\},
$$

equipped with the norm

$$
\|f\|_{L_{\varphi}(\mu)}=\inf \left\{\lambda>0: \int \varphi\left(\frac{|f|}{\lambda}\right) d \mu \leq 1\right\} .
$$

If $\varphi_{0}$ and $\varphi_{1}$ are $N$-functions that satisfy the $\Delta_{2}$-property, and $0<\theta<1$, then we have

$$
\left(L_{\varphi_{0}}(\mu), L_{\varphi_{1}}(\mu)\right)_{\theta}=L_{\varphi}(\mu)
$$

where $\varphi^{-1}=\left(\varphi_{0}^{-1}\right)^{1-\theta}\left(\varphi_{1}^{-1}\right)^{\theta}$, with equivalent norms (see Theorem 7.3 in [GP77]).

Note that in the examples above, unlike the $L_{p}$ space interpolation scale, the interpolation space found is a Sobolev or Orlicz space up to isomorphism. 


\section{Chapter 3}

\section{Uniform homeomorphisms between unit spheres of interpolation spaces}

This chapter is focused on proving the main result: If $\left(A_{0}, A_{1}\right)$ is a compatible couple of uniformly convex spaces, Daher shows in [Dah95] that for every $0<\theta_{1}, \theta_{2}<1$ there is a uniform homeomorphism between the unit spheres of the complex interpolation spaces $A_{\theta_{1}}$ and $A_{\theta_{2}}$.

In [Dah95], the author defines the complex interpolation space using the holomorphic functions over $\mathbb{S}$ but replacing the continuity of the operators over $\overline{\mathbb{S}}$ by a condition of $L_{p}$ integrability. This allows to find on certain hypotheses a minimal representation for each point of the interpolation space sphere in a uniformly continuous way. The idea behind the proofs is to extend classical results of complex analysis to the setting of vector valued functions.

We also present in this chapter the construction of the minimal functions in the interpolation scales of $L_{p}$ and weighted $L_{p}$ spaces. In the last section of this chapter, we present the concept of horizontal maps and some symmetries that can be observed in the theory.

\subsection{Definitions and Notations}

We will fix in this section some notations that will be used throughout the entire text. Consider $\bar{A}=\left(A_{0}, A_{1}\right)$ a compatible couple of Banach spaces.

For all $s+i t \in \overline{\mathbb{S}}$ and for all $\tau \in \mathbb{R}$ we define, for $j=0,1$,

$$
Q_{j}(s+i t, \tau)=\frac{e^{-\pi(\tau-t)} \sin (\pi s)}{\sin ^{2}(\pi s)+\left(\cos (\pi s)-e^{j i \pi-\pi(\tau-t)}\right)^{2}} .
$$

Note that $Q_{j}$ is nonnegative and for $0<\theta<1$

$$
\int_{\mathbb{R}} Q_{0}(\theta, t) d t=1-\theta, \quad \int_{\mathbb{R}} Q_{1}(\theta, t) d t=\theta
$$

(see [Wid61]). Consider $d \mu_{z, j}(t)=Q_{j}(z, t) d t$. 
Definition 3.1.1. ([Dah95]) For all $p \in[1,+\infty]$ and $0<\theta<1$, consider $\mathcal{F}_{\theta}^{p}(\bar{A})$ as the space of functions $f: \overline{\mathbb{S}} \longrightarrow A_{0}+A_{1}$, such that $f$ is holomorphic over $\mathbb{S}$, the functions $t \longmapsto F(j+i t)$ are (Bochner) measurable from $\mathbb{R}$ into $A_{j}(j=0,1)$, and

$$
f(z)=\int_{\mathbb{R}} f(i t) d \mu_{z, 0}(t)+\int_{\mathbb{R}} f(1+i t) d \mu_{z, 1}(t), \text { for all } z \in \mathbb{S},
$$

such that the norm

$$
\begin{gathered}
\|f\|_{\mathcal{F}_{\theta}^{p}(\bar{A})}^{p}=\int_{\mathbb{R}}\|f(i t)\|_{A_{0}}^{p} d \mu_{\theta, 0}(t)+\int_{\mathbb{R}}\|f(1+i t)\|_{A_{1}}^{p} d \mu_{\theta, 1}(t), 1 \leq p<\infty, \\
\|f\|_{\mathcal{F}^{\infty}(\bar{A})}=\max _{j=0,1}\left\{\underset{t \in \mathbb{R}}{\operatorname{esssup}}\|f(j+i t)\|_{A_{j}}\right\}, p=\infty,
\end{gathered}
$$

is finite.

Note that the space $\mathcal{F}^{\infty}(\bar{A})$ no longer depends on $\theta$.

The space $A_{\theta}^{p}$ is defined as

$$
A_{\theta}^{p}=\left\{f(\theta): f \in \mathcal{F}_{\theta}^{p}(\bar{A})\right\},
$$

that becomes a Banach space with the norm

$$
\|a\|_{A_{\theta}^{p}}=\inf \left\{\|f\|_{\mathcal{F}_{\theta}^{p}(\bar{A})}: f \in \mathcal{F}_{\theta}^{p}(\bar{A}), f(\theta)=a\right\} .
$$

We denote by $\mathbb{D}$ the unit open disk centered at the origin in $\mathbb{C}, \mathbb{T}=\partial \mathbb{D}$ the boundary of the disk and $m$ the normalized Lesbesgue measure over $\mathbb{T}$. Recall that, for all $r \in(0,1)$ and for all $\varphi \in \mathbb{T}$, the Poisson kernel for the unit disk is given by

$$
P_{r}(\varphi)=\frac{1-r^{2}}{r^{2}-2 r \cos \varphi+1} .
$$

If $z=r e^{i \varphi} \in \mathbb{D}$ put $P_{z}(t)=P_{r}(\varphi-t)$.

Denote $I_{0}=\left\{e^{i t} \in \mathbb{T} ; 0 \leq t \leq \frac{\pi}{2}\right\} \cup\left\{e^{i t} \in \mathbb{T} ; \frac{3 \pi}{2} \leq t \leq 2 \pi\right\}, I_{1}=\mathbb{T} \backslash I_{0}$; if $t \in I_{0}$ put $A_{t}=A_{0}$ and if $t \in I_{1}$ put $A_{t}=A_{1}$.

Definition 3.1.2. ([Dah95]) The space $\mathcal{H}(\bar{A})$ is defined as the space of all the functions $g: \overline{\mathbb{D}} \longrightarrow$ $A_{0}+A_{1}$ such that $g$ is holomorphic over $\mathbb{D}$ and continuous and bounded over $\overline{\mathbb{D}}$, such that $t \longmapsto g\left(e^{i t}\right)$ is continuous from $\overline{I_{j}}$ into $A_{j}, j=0,1$, and $g(i)=g(-i)=0 . \mathcal{H}(\bar{A})$ is equipped with the norm

$$
\|g\|_{\mathcal{H}(\bar{A})}=\max _{j=0,1}\left\{\sup _{t \in I_{j}}\left\|g\left(e^{i t}\right)\right\|_{A_{j}}\right\} .
$$

For all $z_{0} \in \mathbb{D}$, the space $B_{z_{0}}$ is defined as

$$
B_{z_{0}}=\left\{g\left(z_{0}\right): g \in \mathcal{H}(\bar{A})\right\}
$$


with the norm

$$
\|b\|_{B_{z_{0}}}=\inf \left\{\|g\|_{\mathcal{H}(\bar{A})}: g \in \mathcal{H}(\bar{A}), g\left(z_{0}\right)=b\right\} .
$$

For all $z_{0} \in \mathbb{D}$, consider $d \nu_{z_{0}}(t)=P_{z_{0}}(t) d m(t)$ over $\mathbb{T}$.

Definition 3.1.3. ([Dah95]) For all $p \in[1,+\infty]$, consider $\mathcal{H}_{z_{0}}^{p}(\bar{A})$ as the space of functions $g: \overline{\mathbb{D}} \longrightarrow$ $A_{0}+A_{1}$ such that $g$ is holomorphic over $\mathbb{D}, t \longmapsto g\left(e^{i t}\right)$ are measurable from $I_{j}$ into $A_{j}, j=0,1$, and

$$
g(z)=\int_{\mathbb{T}} g\left(e^{i t}\right) d \nu_{z}(t), \text { for all } z \in \mathbb{D},
$$

equipped with the norm

$$
\begin{aligned}
& \|g\|_{\mathcal{H}_{z_{0}}^{p}(\bar{A})}^{p}=\int_{\mathbb{T}}\left\|g\left(e^{i t}\right)\right\|_{A_{t}}^{p} d \nu_{z_{0}}(t), 1 \leq p<\infty, \\
& \|g\|_{\mathcal{H}^{\infty}(\bar{A})}=\max _{j=0,1}\left\{\underset{t \in I_{j}}{\operatorname{ess} \sup }\left\|g\left(e^{i t}\right)\right\|_{A_{j}}\right\}, p=\infty .
\end{aligned}
$$

The space $B_{z_{0}}^{p}$ is defined as

$$
B_{z_{0}}^{p}=\left\{g\left(z_{0}\right): g \in \mathcal{H}_{z_{0}}^{p}(\bar{A})\right\}
$$

equipped with the norm

$$
\|b\|_{B_{z_{0}}^{p}}=\inf \left\{\|g\|_{\mathcal{H}_{z_{0}}^{p}(\bar{A})}: g \in \mathcal{H}_{z_{0}}^{p}(\bar{A}), g\left(z_{0}\right)=b\right\}
$$

Since in this setting we are considering vector-valued functions, it is important to notice that the notions of measurability and integrability are considered in the Bochner sense as defined in Section 1.5 .

Lemma 3.1.4. For $1 \leq p \leq \infty, \mathcal{H}_{z_{0}}^{p}(\bar{A})$ is isometric to a closed subspace of $L_{p}\left(I_{0}, \nu_{z_{0}}, A_{0}\right) \oplus^{p}$ $L_{p}\left(I_{1}, \nu_{z_{0}}, A_{1}\right)$.

Proof. Note that it follows from the definition of $\mathcal{H}_{z_{0}}^{p}(\bar{A})$ that for each $g \in \mathcal{H}_{z_{0}}^{p}(\bar{A}), g \uparrow_{I_{j}} \in L_{p}\left(I_{j}, \nu_{z_{0}}, A_{j}\right)$, $j=0,1$. Thus, consider the mapping

$$
\begin{aligned}
\Psi: \mathcal{H}_{z_{0}}^{p}(\bar{A}) & \longrightarrow L_{p}\left(I_{0}, \nu_{z_{0}}, A_{0}\right) \oplus^{p} L_{p}\left(I_{1}, \nu_{z_{0}}, A_{1}\right) \\
g & \longmapsto\left(g \uparrow_{I_{0}}, g \uparrow_{I_{1}}\right)
\end{aligned}
$$

Note that $\Psi$ is linear. Moreover, for every $g \in \mathcal{H}_{z_{0}}^{p}(\bar{A})$,

$$
\begin{aligned}
\|\Psi(g)\|_{L_{p}\left(I_{0}, \nu_{z_{0}}, A_{0}\right) \oplus^{p} L_{p}\left(I_{1}, \nu_{z_{0}}, A_{1}\right)}^{p} & =\int_{I_{0}}\left\|g \uparrow_{I_{0}}\left(e^{i t}\right)\right\|_{A_{0}}^{p} d \nu_{z_{0}}(t)+\int_{I_{0}}\left\|g \uparrow_{I_{1}}\left(e^{i t}\right)\right\|_{A_{1}}^{p} d \nu_{z_{0}}(t) \\
& =\int_{\mathbb{T}}\left\|g\left(e^{i t}\right)\right\|_{A_{t}}^{p} d \nu_{z_{0}}(t)=\|g\|_{\mathcal{H}_{z_{0}}^{p}(\bar{A})}^{p}
\end{aligned}
$$


Let $Y \doteq \Psi\left(\mathcal{H}_{z_{0}}^{p}(\bar{A})\right)$. Let us show that $Y$ is closed. Consider the application

$$
\begin{aligned}
\Phi: L_{p}\left(I_{0}, \nu_{z_{0}}, A_{0}\right) \oplus^{p} L_{p}\left(I_{1}, \nu_{z_{0}}, A_{1}\right) & \longrightarrow L_{p}\left(\mathbb{T}, m, A_{0}+A_{1}\right) \\
(f, g) & \longmapsto h_{(f, g)}
\end{aligned}
$$

where

$$
h_{(f, g)}\left(e^{i t}\right)= \begin{cases}f\left(e^{i t}\right) & \text { if } t \in I_{0} \\ g\left(e^{i t}\right) & \text { if } t \in I_{1}\end{cases}
$$

Note that $\Phi$ is linear and injective. Moreover, if $z_{0}=r e^{i t} \in \mathbb{D}$, we have

$$
\frac{1-r}{1+r} \leq P_{z_{0}}(t), \forall t \in \mathbb{T}
$$

Thus, given $(f, g) \in L_{p}\left(I_{0}, \nu_{z_{0}}, A_{0}\right) \oplus^{p} L_{p}\left(I_{1}, \nu_{z_{0}}, A_{1}\right)$, we have

$$
\begin{aligned}
\|\Phi(f, g)\|_{L_{p}\left(\mathbb{T}, m, A_{0}+A_{1}\right)}^{p} & =\int_{\mathbb{T}}\left\|h_{(f, g)}\left(e^{i t}\right)\right\|_{A_{0}+A_{1}}^{p} d m(t) \leq \int_{I_{0}}\left\|f\left(e^{i t}\right)\right\|_{A_{0}}^{p} d m(t)+\int_{I_{1}}\left\|g\left(e^{i t}\right)\right\|_{A_{1}}^{p} d m(t) \\
& \leq \frac{1+r}{1-r}\left(\int_{I_{0}}\left\|f\left(e^{i t}\right)\right\|_{A_{0}}^{p} \frac{1-r}{1+r} d m(t)+\int_{I_{1}}\left\|g\left(e^{i t}\right)\right\|_{A_{1}}^{p} \frac{1-r}{1+r} d m(t)\right) \\
& \leq \frac{1+r}{1-r}\|(f, g)\|_{L_{p}\left(I_{0}, \nu_{z_{0}}, A_{0}\right) \oplus^{p} L_{p}\left(I_{1}, \nu_{z_{0}}, A_{1}\right)}
\end{aligned}
$$

Therefore, $\Phi$ is continuous.

Now, let $\Upsilon: H^{p}\left(A_{0}+A_{1}\right) \longrightarrow L_{p}\left(\mathbb{T}, m, A_{0}+A_{1}\right)$ as in (1.15). We know that $\Upsilon\left(H^{p}\left(A_{0}+A_{1}\right)\right)$ is closed in $L_{p}\left(\mathbb{T}, m, A_{0}+A_{1}\right)$ and

$\Upsilon\left(H^{p}\left(A_{0}+A_{1}\right)\right)=\left\{h \in L_{p}\left(\mathbb{T}, m, A_{0}+A_{1}\right): z \mapsto \int_{\mathbb{T}} h\left(e^{i t}\right) d \nu_{z}(t)\right.$ defines a holomorphic function on $\left.\mathbb{D}\right\}$

Let $\left(f_{n}\right)$ be a sequence on $Y$ and consider $f \in L_{p}\left(I_{0}, \nu_{z_{0}}, A_{0}\right) \oplus^{p} L_{p}\left(I_{1}, \nu_{z_{0}}, A_{1}\right)$ such that $f_{n} \longrightarrow f$. Since $\Phi$ is continuous, we have that $\Phi\left(f_{n}\right) \longrightarrow \Phi(f)$ on $L_{p}\left(\mathbb{T}, m, A_{0}+A_{1}\right)$. Note that $\Phi\left(f_{n}\right) \in$ $\Upsilon\left(H^{p}\left(A_{0}+A_{1}\right)\right)$, which implies that $\Phi(f) \in \Upsilon\left(H^{p}\left(A_{0}+A_{1}\right)\right)$, thus, $f \in Y$, and we have that $Y$ is closed.

If

$$
\begin{aligned}
\Psi: \mathcal{H}_{z_{0}}^{p}(\bar{A}) & \longrightarrow A_{0}+A_{1} \\
g & \longmapsto g\left(z_{0}\right),
\end{aligned}
$$

note that $B_{z_{0}}$ is isometric to $\mathcal{H}_{z_{0}}^{p}(\bar{A}) / \operatorname{ker} \Psi$, thus, $B_{z_{0}}$ is a Banach space.

Recall that the complex logarithm of $0 \neq z \in \mathbb{C}$ is defined as some $w \in \mathbb{C}$ such that $e^{w}=z$. Thus, if $w=x+i y$ we have

$$
e^{w}=z \Leftrightarrow \log z \doteq w \Leftrightarrow e^{x}=|z|, e^{i y}=\frac{z}{|z|} .
$$

From the last part of (3.1) we have that $x=\log |z|$ and $e^{i y}$ is a complex number with modulus 1, thus, the equation $e^{i y}=\frac{z}{|z|}$ has infinitely many solutions which differ from each other by multiples of $2 \pi i$. If we consider the polar form of $z$, writing $z=r e^{i s}$, we have $z=r e^{i s}=r e^{i(s+2 k \pi)}$ for all $k \in \mathbb{Z}$; 
therefore,

$$
w=\log z=\log |z|+i(s+2 k \pi),
$$

for $k \in \mathbb{Z}$ (see Section 3.4 in [Ahl66] for more details and properties). Throughout this text, we will consider $k=0$, that is, given $0 \neq z=r e^{i s} \in \mathbb{C}$, we put $\log z=\log |z|+i s$.

In what follows, we will present some calculations so that we can better understand the behaviour and properties of the spaces defined above.

Consider the application $\Lambda: \overline{\mathbb{S}} \longrightarrow \overline{\mathbb{D}}$ given by

$$
\Lambda(z)=\frac{1+i e^{i \pi z}}{i+e^{i \pi z}}
$$

Note that $\Lambda^{\prime}(z)=\frac{-2 i \pi e^{i \pi z}}{\left(i+e^{i \pi z}\right)^{2}} \neq 0$ for all $z \in \mathbb{S}$, thus, $\Lambda$ is a conformal mapping.

Let $0<\theta<1$ and put $z_{0}=\Lambda(\theta)$. In the following paragraphs we will show that the mapping $g \longmapsto g \circ \Lambda$ defines a isometry between $\mathcal{H}(\bar{A})$ and $\mathcal{F}(\bar{A})$ (as in Definition 2.3.1) and between $\mathcal{H}_{z_{0}}^{p}(\bar{A})$ and $\mathcal{F}_{\theta}^{p}(\bar{A})$. Therefore we have that $A_{\theta}^{p}=B_{z_{0}}^{p}$ with equal norms, for $p \in[1,+\infty]$. These isometries will be very useful later, allowing us to choose the most convenient space in the demonstrations.

We will start by showing some properties of the application $\Lambda$. Note that:

1) $|\Lambda(j+i t)|=1$, for all $t \in \mathbb{R}$ and $j=0,1$ :

$$
|\Lambda(j+i t)|=\left|\frac{1+i e^{i \pi j-\pi t}}{i+e^{i \pi j-\pi t}}\right|=\frac{1-i e^{-i \pi j-\pi t}}{-i+e^{-i \pi j-\pi t}} \frac{1+i e^{i \pi j-\pi t}}{i+e^{i \pi j-\pi t}}=1
$$

2) $\mathfrak{R}(\Lambda(i t))>0$, for all $t \in \mathbb{R}$ :

$$
\Lambda(i t)=\frac{1+i e^{-\pi t}}{i+e^{-\pi t}} \frac{-i+e^{-\pi t}}{-i+e^{-\pi t}}=\frac{-i+2 e^{-\pi t}+i e^{-2 \pi t}}{1+e^{-2 \pi t}}
$$

and then

$$
\mathfrak{R}(\Lambda(i t))=\frac{2 e^{-\pi t}}{1+e^{-2 \pi t}}>0
$$

3) $\mathfrak{R}(\Lambda(1+i t))<0$, for all $t \in \mathbb{R}$ :

$$
\Lambda(1+i t)=\frac{1+i e^{i \pi-\pi t}}{i+e^{i \pi-\pi t}} \frac{-i+e^{-i \pi-\pi t}}{-i+e^{-i \pi-\pi t}}=\frac{-i-2 e^{-\pi t}+i e^{-2 \pi t}}{1+e^{-2 \pi t}}
$$

and then

$$
\mathfrak{R}(\Lambda(1+i t))=\frac{-2 e^{-\pi t}}{1+e^{-2 \pi t}}<0
$$

Note that this shows that $\{\Lambda(j+i t): t \in \mathbb{R}\} \subseteq\left\{e^{i s}: s \in I_{j}\right\}$, for $j=0,1$. On the other hand, if $s \in I_{j}$, $j=0,1$, put $t=\frac{1}{\pi} \log \left(\frac{e^{i \pi j}\left(1+i e^{i s}\right)}{e^{i s}+i}\right) \in \mathbb{R}$, then we have $e^{i s}=\Lambda(j+i t)$. Thus,

$$
\{\Lambda(j+i t): t \in \mathbb{R}\}=\left\{e^{i s}: s \in I_{j}\right\}, \text { for } j=0,1 .
$$

Note also that for $s \in(0,1)$ we have $\Lambda(s)=\frac{1+i e^{i \pi s}}{i+e^{i \pi s}} \in \mathbb{R}$ (in particular, $\Lambda(1 / 2)=0$ ). The above properties can be seen in the figure below: 

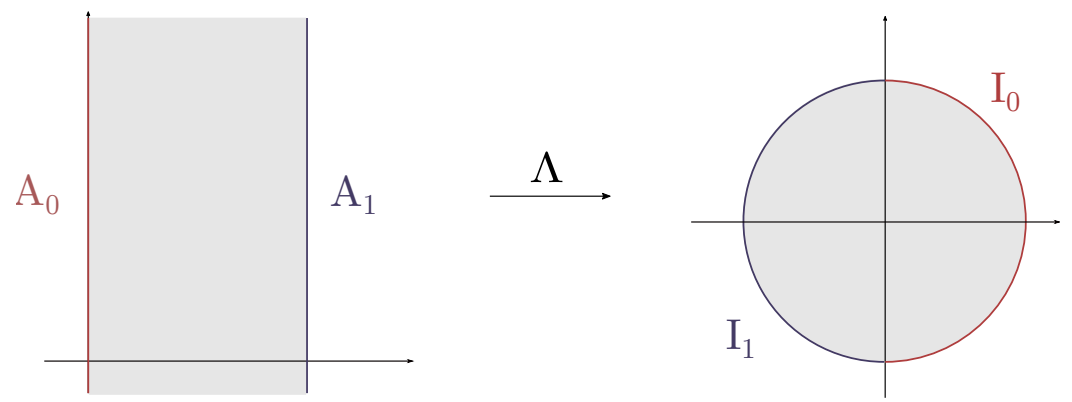

Let us show now that the mapping

$$
\begin{aligned}
\Upsilon: \mathcal{H}(\bar{A}) & \longrightarrow \mathcal{F}(\bar{A}) \\
g & \longmapsto g \circ \Lambda
\end{aligned}
$$

is a surjective isometry. Note first that if $g \in \mathcal{H}(\bar{A})$ then $g \circ \Lambda \in \mathcal{F}(\bar{A})$, indeed,

(i) $g \circ \Lambda$ is continuous on $\overline{\mathbb{S}}$ : it follows from the continuity of $g$ and $\Lambda$.

(ii) $g \circ \Lambda$ is bounded on $\overline{\mathbb{S}}$ : it follows from the fact that $g$ is bounded over $\overline{\mathbb{D}}$.

(iii) $g \circ \Lambda$ is holomorphic on $\mathbb{S}$ : it follows from the properties of holomorphic functions.

(iv) The functions $t \longmapsto g(\Lambda(j+i t)), j=0,1$, are continuous from $\mathbb{R}$ into $A_{j}$ : Note that

$$
\begin{aligned}
& \mathbb{R} \longrightarrow I_{j} \\
& t \longmapsto \Lambda(j+i t)=\frac{1+i e^{i \pi j-\pi t}}{i+e^{i \pi j-\pi t}}
\end{aligned}
$$

is a continuous function in $t$. Then, if $t \rightarrow t_{0}$ we have that $e^{i s}:=\Lambda(j+i t) \rightarrow e^{i s_{0}}:=\Lambda\left(j+i t_{0}\right)$, which implies that $s \rightarrow s_{0}$, and then $g\left(e^{i s}\right) \rightarrow g\left(e^{i s_{0}}\right)$ in $A_{j}$.

(v) $\|g(\Lambda(j+i t))\|_{A_{j}} \rightarrow 0$ when $|t| \rightarrow+\infty(j=0,1)$ : We have that

$$
\begin{gathered}
\Lambda(i t)=\frac{-i+2 e^{-\pi t}+i e^{-2 \pi t}}{1+e^{-2 \pi t}} \rightarrow-i, \text { when } t \rightarrow+\infty \\
\Lambda(1+i t)=\frac{-i-2 e^{-\pi t}+i e^{-2 \pi t}}{1+e^{-2 \pi t}} \rightarrow-i, \text { when } t \rightarrow+\infty \\
\Lambda(i t)=\frac{-i+2 e^{-\pi t}+i e^{-2 \pi t}}{1+e^{-2 \pi t}} \rightarrow i, \text { when } t \rightarrow-\infty \\
\Lambda(1+i t)=\frac{-i-2 e^{-\pi t}+i e^{-2 \pi t}}{1+e^{-2 \pi t}} \rightarrow i, \text { when } t \rightarrow-\infty
\end{gathered}
$$

and then by item (iv) and the fact that $g(i)=g(-i)=0$, the result follows.

(vi) Given $f \in \mathcal{F}(\bar{A})$ and taking $g=f \circ \Lambda^{-1} \in \mathcal{H}(\bar{A})$, we have that $\Upsilon(g)=g \circ \lambda=f$, and thus, $\Upsilon$ is surjective. 
Note finally that

$$
\|g \circ \Lambda\|_{\mathcal{F}(\bar{A})}=\max _{j=0,1}\left\{\sup _{t \in \mathbb{R}}\|g(\Lambda(j+i t))\|_{A_{j}}\right\}=\max _{j=0,1}\left\{\sup _{t \in I_{j}}\left\|g\left(e^{i t}\right)\right\|_{A_{j}}\right\}=\|g\|_{\mathcal{H}(\bar{A})},
$$

and we have that the spaces $\mathcal{H}(\bar{A})$ and $\mathcal{F}(\bar{A})$ are isometric (which implies that $\mathcal{H}(\bar{A})$ is a Banach space).

Now, let us show now that the mapping

$$
\begin{aligned}
\Xi: \mathcal{H}_{z_{0}}^{p}(\bar{A}) & \longrightarrow \mathcal{F}_{\theta}^{p}(\bar{A}) \\
g & \longmapsto g \circ \Lambda
\end{aligned}
$$

is a surjective isometry. It is immediate that $g \circ \Lambda$ is holomorphic on $\mathbb{S}$ and $t \longmapsto g(\Lambda(j+i t)) \in A_{j}$ are Bochner-measurable for $j=0,1$. Moreover, note that, for $z \in \mathbb{S}$ we have

$$
g(\Lambda(z))=\int_{\mathbb{T}} g\left(e^{i t}\right) d \nu_{\Lambda(z)}(t)=\int_{\mathbb{R}} g(\Lambda(i t)) d \mu_{z, 0}(t)+\int_{\mathbb{R}} g(\Lambda(1+i t)) d \mu_{z, 1}(t)
$$

Therefore, $g \circ \Lambda \in \mathcal{F}_{\theta}^{p}(\bar{A})$.

If we consider $z_{0}=r_{0} e^{i \varphi_{0}}$, we have

$$
\frac{1+i e^{i \pi \theta}}{i+e^{i \pi \theta}}=\Lambda(\theta)=z_{0}=r_{0} e^{i \varphi_{0}}
$$

Note that

$$
\begin{gathered}
\Lambda(\theta)=\frac{1+i e^{i \pi \theta}}{i+e^{i \pi \theta}}=\frac{\cos (\pi \theta)}{1+\sin (\pi \theta)} \in \mathbb{R} \Rightarrow|\Lambda(\theta)|=r_{0} \\
r_{0}^{2}=\Lambda(\theta) \overline{\Lambda(\theta)}=\frac{\cos ^{2}(\pi \theta)}{(1+\sin (\pi \theta))^{2}}=\frac{1-\sin (\pi \theta)}{1+\sin (\pi \theta)}
\end{gathered}
$$

Without loss of generality, we can consider $\varphi_{0}=0$. Thus,

$$
\begin{aligned}
\|g\|_{\mathcal{H}_{z_{0}}^{p}(\bar{A})}^{p}= & \int_{\mathbb{T}}\left\|g\left(e^{i t}\right)\right\|_{A_{t}}^{p} d \nu_{z_{0}}(t)=\int_{\mathbb{T}}\left\|g\left(e^{i t}\right)\right\|_{A_{t}}^{p} P_{z_{0}}(t) d m(t) \\
= & \int_{\mathbb{T}}\left\|g\left(e^{i t}\right)\right\|_{A_{t}}^{p} P_{r_{0}}(-t) d m(t)=\int_{\mathbb{T}}\left\|g\left(e^{i t}\right)\right\|_{A_{t}}^{p}\left(\frac{1-r_{0}^{2}}{r_{0}^{2}-2 r_{0} \cos (-t)+1}\right) d m(t) \\
\|g \circ \Lambda\|_{\mathcal{F}_{\theta}^{p}(\bar{A})}^{p} & =\int_{\mathbb{R}}\|g(\Lambda(i s))\|_{A_{0}}^{p} d \mu_{\theta, 0}(s)+\int_{\mathbb{R}}\|g(\Lambda(1+i s))\|_{A_{1}}^{p} d \mu_{\theta, 1}(s) \\
& =\int_{\mathbb{R}}\|g(\Lambda(i s))\|_{A_{0}}^{p} \frac{e^{-\pi s} \sin (\pi \theta)}{\sin ^{2}(\pi \theta)+\left(\cos (\pi \theta)-e^{-\pi s}\right)^{2}} d s \\
& +\int_{\mathbb{R}}\|g(\Lambda(1+i s))\|_{A_{1}}^{p} \frac{e^{-\pi s} \sin (\pi \theta)}{\sin ^{2}(\pi \theta)+\left(\cos (\pi \theta)-e^{-\pi s-i \pi}\right)^{2}} d s \\
& =\int_{\mathbb{R}}\|g(\Lambda(i s))\|_{A_{0}}^{p} \frac{\sin (\pi \theta)}{2(\cosh (\pi s)-(\cos (\pi \theta))} d s \\
& +\int_{\mathbb{R}}\|g(\Lambda(1+i s))\|_{A_{1}}^{p} \frac{\sin (\pi \theta)}{2(\cosh (\pi s)+(\cos (\pi \theta))} d s
\end{aligned}
$$


For $t \in \mathbb{T}$, we have

$$
\frac{1-r_{0}^{2}}{r_{0}^{2}-2 r_{0} \cos (-t)+1}=\frac{1-\frac{1-\sin (\pi \theta)}{1+\sin (\pi \theta)}}{\frac{1-\sin (\pi \theta)}{1+\sin (\pi \theta)}-\frac{2|\cos (\pi \theta)|}{1+\sin (\pi \theta)} \cos (-t)+1}=\frac{\sin (\pi \theta)}{1-|\cos (\pi \theta)| \cos (-t)}
$$

For $t \in I_{0}$, there is $s \in \mathbb{R}$ such that $e^{i t}=\Lambda(i s)$; namely $t=-i \ln \left(\frac{1-i e^{\pi s}}{e^{\pi s}-i}\right)$. Thus,

$$
\begin{gathered}
\cos (-t)=\frac{1}{\cosh (\pi s)}=\operatorname{sech}(\pi s) \\
\frac{1-r_{0}^{2}}{r_{0}^{2}-2 r_{0} \cos (-t)+1}=\frac{\sin (\pi \theta)}{1-\frac{\cos (\pi \theta)}{\cosh (\pi s)}}=\frac{\cosh (\pi s) \sin (\pi \theta)}{\cosh (\pi s)-\cos (\pi \theta)} \\
d m(t)=\frac{1}{2 \pi} \frac{2 \pi e^{\pi s}}{e^{2 \pi s}+1} d s=\frac{1}{2 \cosh (\pi s)} d s
\end{gathered}
$$

For $t \in I_{1}$, there is $s \in \mathbb{R}$ such that $e^{i t}=\Lambda(1+i s)$; namely $t=-i \ln \left(\frac{-i\left(e^{\pi s}-i\right)}{e^{\pi s}+i}\right)$. Hence,

$$
\begin{gathered}
\cos (-t)=-\frac{1}{\cosh (\pi s)}=-\operatorname{sech}(\pi s) \\
\frac{1-r_{0}^{2}}{r_{0}^{2}-2 r_{0} \cos (-t)+1}=\frac{\sin (\pi \theta)}{1+\frac{\cos (\pi \theta)}{\cosh (\pi s)}}=\frac{\cosh (\pi s) \sin (\pi \theta)}{\cosh (\pi s)+\cos (\pi \theta)} \\
d m(t)=\frac{1}{2 \pi} \frac{-2 \pi e^{\pi s}}{e^{2 \pi s}+1} d s=-\frac{1}{2 \cosh (\pi s)} d s
\end{gathered}
$$

Thus,

$$
\begin{aligned}
\|g\|_{\mathcal{H}_{z_{0}}^{p}(\bar{A})}^{p} & =\int_{\mathbb{T}}\left\|g\left(e^{i t}\right)\right\|_{A_{t}}^{p}\left(\frac{1-r_{0}^{2}}{r_{0}^{2}-2 r_{0} \cos (-t)+1}\right) d m(t) \\
& =\int_{\mathbb{R}}\|g(\Lambda(i s))\|_{A_{0}}^{p} \frac{\sin (\pi \theta)}{2(\cosh (\pi s)-(\cos (\pi \theta))} d s \\
& +\int_{\mathbb{R}}\|g(\Lambda(1+i s))\|_{A_{1}}^{p} \frac{\sin (\pi \theta)}{2(\cosh (\pi s)+(\cos (\pi \theta))} d s=\|g \circ \Lambda\|_{\mathcal{F}_{\theta}^{p}(\bar{A})}^{p},
\end{aligned}
$$

which implies that $g \longmapsto g \circ \Lambda$ defines a isometry between $\mathcal{H}_{z_{0}}^{p}(\bar{A})$ and $\mathcal{F}_{\theta}^{p}(\bar{A})$. Given $f \in \mathcal{F}_{\theta}^{p}(\bar{A})$ and taking $g=f \circ \Lambda^{-1} \in \mathcal{H}_{z_{0}}^{p}(\bar{A})$, we have that $\Xi(g)=g \circ \lambda=f$, and thus, $\Xi$ is surjective. Therefore, we have

$$
B_{z_{0}}^{p}=\left\{g\left(z_{0}\right): g \in \mathcal{H}_{z_{0}}^{p}(\bar{A})\right\}=\left\{g(\Lambda(\theta)): g \in \mathcal{H}_{z_{0}}^{p}(\bar{A})\right\}=\left\{f(\theta): f \in \mathcal{F}_{\theta}^{p}(\bar{A})\right\}=A_{\theta}^{p}
$$

And for all $a \in B_{z_{0}}^{p}=A_{\theta}^{p}$,

$$
\begin{aligned}
\|a\|_{B_{z_{0}}^{p}} & =\inf \left\{\|g\|_{\mathcal{H}_{z_{0}}^{p}(\bar{A})}: g \in \mathcal{H}_{z_{0}}^{p}(\bar{A}), g\left(z_{0}\right)=a\right\}=\inf \left\{\|g \circ \Lambda\|_{\mathcal{F}_{\theta}^{p}(\bar{A})}: g \in \mathcal{H}_{z_{0}}^{p}(\bar{A}), g(\Lambda(\theta))=a\right\} \\
& =\inf \left\{\|f\|_{\mathcal{H}_{z_{0}}^{p}(\bar{A})}: f \in \mathcal{F}_{\theta}^{p}(\bar{A}), f(\theta)=a\right\}=\|a\|_{A_{\theta}^{p}} .
\end{aligned}
$$

Now, let us see some properties of the space $\mathcal{H}_{z_{0}}^{p}(\bar{A})$. 
Proposition 3.1.5. If $A_{0}$ and $A_{1}$ are reflexive, then $\mathcal{H}_{z_{0}}^{p}(\bar{A})$ is reflexive for $1<p<\infty$.

Proof. Since $A_{0}$ and $A_{1}$ are reflexive, it follows from Proposition 1.5.11 that $L_{p}\left(I_{0}, \nu_{z_{0}}, A_{0}\right)$ and $L_{p}\left(I_{1}, \nu_{z_{0}}, A_{1}\right)$ are reflexive. Thus, $L_{p}\left(I_{0}, \nu_{z_{0}}, A_{0}\right) \oplus^{p} L_{p}\left(I_{1}, \nu_{z_{0}}, A_{1}\right)$ is reflexive. We have from Proposition 1.1.7(a) that every closed subspace of $L_{p}\left(I_{0}, \nu_{z_{0}}, A_{0}\right) \oplus^{p} L_{p}\left(I_{1}, \nu_{z_{0}}, A_{1}\right)$ is reflexive, therefore, from Lemma 3.1.4, $\mathcal{H}_{z_{0}}^{p}(\bar{A})$ is isomorphic to a reflexive space, and the result follows from Proposition 1.1.7(b).

Proposition 3.1.6. If $A_{0}$ and $A_{1}$ are uniformly convex, then $\mathcal{H}_{z_{0}}^{p}(\bar{A})$ is uniformly convex for $1<p<\infty$.

Proof. Since $A_{0}$ and $A_{1}$ are uniformly convex, it follows from Proposition 1.5.13 that $L_{p}\left(I_{0}, \nu_{z_{0}}, A_{0}\right)$ and $L_{p}\left(I_{1}, \nu_{z_{0}}, A_{1}\right)$ are uniformly convex. Therefore, from Proposition 1.1.17, $L_{p}\left(I_{0}, \nu_{z_{0}}, A_{0}\right) \oplus^{p}$ $L_{p}\left(I_{1}, \nu_{z_{0}}, A_{1}\right)$ is uniformly convex. We have from Proposition 1.1.13(c) that every closed subspace of $L_{p}\left(I_{0}, \nu_{z_{0}}, A_{0}\right) \oplus^{p} L_{p}\left(I_{1}, \nu_{z_{0}}, A_{1}\right)$ is uniformly convex. Thus, from Lemma 3.1.4, $\mathcal{H}_{z_{0}}^{p}(\bar{A})$ is isometric to a uniformly convex space, and the result follows from Proposition 1.1.13(d).

\subsection{Uniform homeomorphism between unit spheres}

Let $\left(A_{0}, A_{1}\right)$ be a compatible Banach couple, $0<\theta<1$ and $\Lambda(\theta)=z_{0}$.

Proposition 3.2.1. For all $1 \leq p<\infty$ we have $B_{z_{0}}^{p}=B_{z_{0}}^{\infty}=A_{\theta}^{\infty}$, with equal norms.

Proof. Note that $\mathcal{H}^{\infty}(\bar{A}) \subseteq \mathcal{H}_{z_{0}}^{p}(\bar{A})$. Thus, for all $a \in B_{z_{0}}^{\infty}$ we have

$$
\|a\|_{B_{z_{0}}^{p}}=\inf \left\{\|g\|_{\mathcal{H}_{z_{0}}^{p}(\bar{A})}: g \in \mathcal{H}_{z_{0}}^{p}(\bar{A}), g\left(z_{0}\right)=a\right\} \leq \inf \left\{\|g\|_{\mathcal{H}^{\infty}(\bar{A})}: g \in \mathcal{H}^{\infty}(\bar{A}), g\left(z_{0}\right)=a\right\}=\|a\|_{B_{z_{0}}^{\infty}} .
$$

On the other hand, consider $\epsilon>0$ and $a \in B_{z_{0}}^{p}$ with $a \neq 0$. Let $g \in \mathcal{H}_{z_{0}}^{p}(\bar{A})$ be such that $g\left(z_{0}\right)=a$ and $\|g\|_{\mathcal{H}_{z_{0}}^{p}(\bar{A})} \leq\|a\|_{B_{z_{0}}^{p}}+\epsilon$. Let $a^{*} \in\left(A_{0}+A_{1}\right)^{*}$ be such that $\left\|a^{*}\right\|=1$ and $a^{*}\left(g\left(z_{0}\right)\right) \neq 0$, and let us write $z_{0}=r e^{i s}$. We have that $a^{*} \circ g: \mathbb{D} \longrightarrow \mathbb{C}$ is holomorphic. Moreover, since $g \in \mathcal{H}_{z_{0}}^{p}(\bar{A})$, we have that $g$ satisfies the vector valued Poisson integral, that is,

$$
g(z)=\int_{\mathbb{T}} g\left(e^{i t}\right) d \nu_{z}(t)=\int_{\mathbb{T}} g\left(e^{i t}\right) P_{z}(t) d m(t)
$$

for all $z \in \mathbb{D}$. From Hille's Theorem (Theorem 1.5.5) it follows that

$$
a^{*}(g(z))=a^{*}\left(\int_{\mathbb{T}} g\left(e^{i t}\right) P_{z}(t) d m(t)\right)=\int_{\mathbb{T}} a^{*}\left(g\left(e^{i t}\right)\right) P_{z}(t) d m(t)
$$

for all $z \in \mathbb{D}$. Thus, according to Theorem 1.3.3, it follows that $a^{*} \circ g \in H^{1}(\mathbb{D})$. From Theorem 1.3.4 and the hypothesis $a^{*}\left(g\left(z_{0}\right)\right) \neq 0$, we have

$$
-\infty<\log \left|a^{*}\left(g\left(z_{0}\right)\right)\right| \leq \frac{1+r}{1-r} \int_{\mathbb{T}} \log \left|a^{*}\left(g\left(e^{i t}\right)\right)\right| d m(t) .
$$


Since for all $t \in \mathbb{T}$ we have

$$
\left|a^{*}\left(g\left(e^{i t}\right)\right)\right| \leq\left\|a^{*}\right\|\left\|g\left(e^{i t}\right)\right\|_{A_{0}+A_{1}} \leq\left\|g\left(e^{i t}\right)\right\|_{A_{t}},
$$

and $\log$ is an increasing function, it follows from (3.2) that

$$
-\infty<\int_{\mathbb{T}} \log \left|a^{*}\left(g\left(e^{i t}\right)\right)\right| d m(t) \leq \int_{\mathbb{T}} \log \left\|g\left(e^{i t}\right)\right\|_{A_{t}} d m(t) .
$$

Hence, if we put $\varphi(t)=\left\|g\left(e^{i t}\right)\right\|_{A_{t}}$ for each $t \in \mathbb{T}$, we have that $\int_{\mathbb{T}} \log \varphi(t) d m(t)>-\infty$. Moreover, $\varphi \in L_{p}(\mathbb{T})$. Indeed, note that for all $t \in \mathbb{T}$ we have

$$
P_{z_{0}}(t)=P_{r}(s-t)=\frac{1-r^{2}}{1-2 r \cos (s-t)+r^{2}} \geq \frac{1-r}{1+r}
$$

thus,

$$
\infty>\|g\|_{\mathcal{H}_{z_{0}}^{p}(\bar{A})}^{p}=\int_{\mathbb{T}}\left\|g\left(e^{i t}\right)\right\|_{A_{t}}^{p} d \nu_{z_{0}}(t)=\int_{\mathbb{T}}\left\|g\left(e^{i t}\right)\right\|_{A_{t}}^{p} P_{z_{0}}(t) d m(t) \geq \frac{1-r}{1+r} \int_{\mathbb{T}}\left\|g\left(e^{i t}\right)\right\|_{A_{t}}^{p} d m(t) .
$$

Furthermore, $\varphi$ is positive. It follows then from Corollary 1.3.10, that there is an outer function $G \in H^{p}(\mathbb{D})$ such that

$$
\left|G\left(e^{i t}\right)\right|=\varphi(t)=\left\|g\left(e^{i t}\right)\right\|_{A_{t}}
$$

for almost every $t \in \mathbb{T}$. Define $H=G\left(z_{0}\right) \frac{g}{G}$. Let us show that $H \in \mathcal{H}^{\infty}(\bar{A})$ :

(i) $H: \mathbb{D} \longrightarrow A_{0}+A_{1}$ is holomorphic: Let $a^{*} \in\left(A_{0}+A_{1}\right)^{*}$. For all $z \in \mathbb{D}$ we have $a^{*}(H(z))=$ $\frac{G\left(z_{0}\right)}{G(z)} a^{*}(g(z))$. Thus, since $a^{*} \circ g: \mathbb{D} \rightarrow \mathbb{C}$ is holomorphic, it follows that $a^{*} \circ H$ is holomorphic.

(ii) $t \longmapsto H\left(e^{i t}\right)$ are measurable from $I_{j}$ to $A_{j}, j=0,1$ : Note first that $\forall t \in I_{j}, H\left(e^{i t}\right)=$ $\frac{G\left(z_{0}\right)}{G\left(e^{i t}\right)} g\left(e^{i t}\right) \in A_{j}$. Moreover, since $I_{j} \ni t \longmapsto \frac{1}{G\left(e^{i t}\right)} \in \mathbb{C}$ is measurable, there is a sequence $\left(G_{n}\right)$ of simple functions such that $\lim _{n \rightarrow \infty} G_{n}\left(e^{i t}\right)=\frac{1}{G\left(e^{i t}\right)}$ for all $t \in I_{j}$. Furthermore, since $I_{j} \ni t \longmapsto g\left(e^{i t}\right) \in A_{j}$ is measurable, there is a sequence $\left(g_{n}\right)$ of simple functions such that $\lim _{n \rightarrow \infty}\left\|g_{n}\left(e^{i t}\right)-g\left(e^{i t}\right)\right\|_{A_{j}}=0$ for almost every $t \in I_{j}$. Note that $H_{n} \doteq G\left(z_{0}\right) G_{n} . g_{n}: I_{j} \longrightarrow A_{j}$ is a simple function for all $n \in \mathbb{N}$. Thus, for almost every $t \in I_{j}$ we have

$$
\begin{aligned}
\left\|H_{n}\left(e^{i t}\right)-H\left(e^{i t}\right)\right\|_{A_{t}} & =\left\|G\left(z_{0}\right) G_{n}\left(e^{i t}\right) g_{n}\left(e^{i t}\right)-G\left(z_{0}\right) \frac{g\left(e^{i t}\right)}{G\left(e^{i t}\right)}\right\|_{A_{t}} \\
& \leq\left|G\left(z_{0}\right)\right|\left\|G_{n}\left(e^{i t}\right) g_{n}\left(e^{i t}\right)-\frac{g_{n}\left(e^{i t}\right)}{G\left(e^{i t}\right)}\right\|_{A_{t}}+\left|G\left(z_{0}\right)\right|\left\|\frac{g_{n}\left(e^{i t}\right)}{G\left(e^{i t}\right)}-\frac{g\left(e^{i t}\right)}{G\left(e^{i t}\right)}\right\|_{A_{t}} \\
& =\left|G\left(z_{0}\right)\right|\left[\left|G_{n}\left(e^{i t}\right)-\frac{1}{G\left(e^{i t}\right)}\right|\left\|g_{n}\left(e^{i t}\right)\right\|_{A_{t}}+\left|\frac{1}{G\left(e^{i t}\right)}\right|\left\|g_{n}\left(e^{i t}\right)-g\left(e^{i t}\right)\right\|_{A_{t}}\right]
\end{aligned}
$$

and we have that $\left\|H_{n}\left(e^{i t}\right)-H\left(e^{i t}\right)\right\|_{A_{t}} \stackrel{n \rightarrow \infty}{\longrightarrow} 0$. Hence $I_{j} \ni t \longmapsto H\left(e^{i t}\right) \in A_{j}$ is measurable.

(iii) $H(z)=\int_{\mathbb{T}} H\left(e^{i t}\right) d \nu_{z}(t)$ for all $z \in \mathbb{D}$ : Let $a^{*} \in\left(A_{0}+A_{1}\right)^{*}$. We will show that $a^{*} \circ H \in H^{1}(\mathbb{D})$, thus, Theorem 1.3.3 implies that $a^{*} \circ H$ satisfies the Poisson integral, and therefore, Hille's Theorem (Theorem 1.5.5) implies the desired result. Note initially that $a^{*} \circ g \in H^{1}(\mathbb{D}$ ) (as seen above). It follows from Theorem 1.3 .8 that $a^{*}(g(z))=B(z) S(z) F(z), \forall z \in \mathbb{D}$, where $B$ 
is a Blaschke product, $S$ is a singular inner function, and $F$ is an outer function such that

$$
F(z)=e^{i \alpha} \exp \left(\int_{\mathbb{T}} \frac{e^{i t}+z}{e^{i t}-z} \log \left|a^{*}\left(g\left(e^{i t}\right)\right)\right| d m(t)\right), z \in \mathbb{D}
$$

for some $\alpha \in \mathbb{R}$. Since $G \in H^{p}(\mathbb{D})$ is an outer function, there are $\beta \in \mathbb{R}, \psi \in L_{p}(\mathbb{T})$ such that $\log \psi \in L_{1}(\mathbb{T}), \psi \geq 0$ and

$$
G(z)=e^{i \beta} \exp \left(\int_{\mathbb{T}} \frac{e^{i t}+z}{e^{i t}-z} \log \psi\left(e^{i t}\right) d m(t)\right), z \in \mathbb{D} .
$$

Note that

$$
\frac{F(z)}{G(z)}=e^{i(\alpha-\beta)} \exp \left(\int_{\mathbb{T}} \frac{e^{i t}+z}{e^{i t}-z} \log \frac{\left|a^{*}\left(g\left(e^{i t}\right)\right)\right|}{\psi\left(e^{i t}\right)} d m(t)\right), z \in \mathbb{D} .
$$

Thus, $F / G$ is an outer function for the space $H^{1}(\mathbb{D})$. Therefore, it follows from Theorem 1.3.8 that $\frac{a^{*} \circ g}{G}=B S \frac{F}{G} \in H^{1}(\mathbb{D})$. Thus,

$$
a^{*} \circ H=a^{*}\left(G\left(z_{0}\right) \frac{g}{G}\right)=G\left(z_{0}\right) \frac{a^{*} \circ g}{G} \in H^{1}(\mathbb{D}),
$$

and we have the desired result.

(iv) Note finally that

$$
\left.\|H\|_{\mathcal{H}^{\infty}(\bar{A})}=\max _{j=0,1}\left\{\|H\|_{L_{\infty}\left(I_{j}, A_{j}\right)}\right)\right\}=\max _{j=0,1}\left\{\underset{t \in I_{j}}{\operatorname{ess} \sup }\left\|G\left(z_{0}\right) \frac{g\left(e^{i t}\right)}{G\left(e^{i t}\right)}\right\|_{A_{t}}\right\}=\left|G\left(z_{0}\right)\right|<\infty .
$$

Thus, $H \in \mathcal{H}^{\infty}(\bar{A})$ and $H\left(z_{0}\right)=a \in B_{z_{0}}^{\infty}$. Consequently,

$$
\|H\|_{\mathcal{H}^{\infty}(\bar{A})}^{p} \leq\left|G\left(z_{0}\right)\right|^{p} \leq \int_{\mathbb{T}}\left|G\left(e^{i t}\right)\right|^{p} d \nu_{z_{0}}(t) \leq \int_{\mathbb{T}}\left\|g\left(e^{i t}\right)\right\|_{A_{t}}^{p} d \nu_{z_{0}}(t)=\|g\|_{\mathcal{H}_{z_{0}}^{p}(\bar{A})}^{p} .
$$

Therefore, $\|H\|_{\mathcal{H}^{\infty}(\bar{A})} \leq\|g\|_{\mathcal{H}_{z_{0}}^{p}(\bar{A})} \leq\|a\|_{B_{z_{0}}^{p}}+\epsilon$. Thus, $\|a\|_{B_{z_{0}}^{\infty}} \leq\|a\|_{B_{z_{0}}^{p}}$ and we have the desired result.

Proposition 3.2.2. $A_{\theta}=A_{\theta}^{\infty}$, with equal norms.

Proof. Let us show that $X=A_{\theta}^{\infty}$ and $Y=A_{\theta}$ satisfy the conditions of Lemma 1.1.2.

(i) $A_{\theta}$ is a subspace of $A_{\theta}^{\infty}$ : Let $a \in A_{\theta}$, and consider $f \in \mathcal{F}(\bar{A})$ such that $f(\theta)=a$. Let us show that $f \in \mathcal{F}^{\infty}(\bar{A})$. We already know that $f: \overline{\mathbb{S}} \longrightarrow A_{0}+A_{1}$ is holomorphic on $\mathbb{S}$. Note that, since $t \longmapsto f(j+i t) \in A_{j}, j=0,1$, is continuous, it follows that it is Bochner-measurable. Moreover, from Remark 1.5.9, it follows that for all $z \in \mathbb{S}$ we have

$$
f(z)=\int_{\mathbb{R}} f(i t) d \mu_{z, 0}(t)+\int_{\mathbb{R}} f(1+i t) d \mu_{z, 1}(t) .
$$

And we have $\|f\|_{\mathcal{F}^{\infty}(\bar{A})}=\|f\|_{\mathcal{F}(\bar{A})}<\infty$. Thus, $f \in \mathcal{F}^{\infty}(\bar{A})$ and $f(\theta)=a \in A_{\theta}^{\infty}$. 
(ii) $\|a\|_{A_{\theta}^{\infty}} \leq\|a\|_{A_{\theta}}$ for all $a \in A_{\theta}$ : Indeed, since $\mathcal{F}(\bar{A}) \subseteq \mathcal{F}^{\infty}(\bar{A})$, we have

$$
\|a\|_{A_{\theta}^{\infty}}=\inf \left\{\|f\|_{\mathcal{F}^{\infty}(\bar{A})}: f \in \mathcal{F}^{\infty}(\bar{A}), f(\theta)=a\right\} \leq \inf \left\{\|f\|_{\mathcal{F}(\bar{A})}: f \in \mathcal{F}(\bar{A}), f(\theta)=a\right\}=\|a\|_{A_{\theta}} .
$$

(iii) $B_{A_{\theta}}$ is dense in $B_{A_{\theta}^{\infty}}$ : Let $a \in A_{\theta}^{\infty}$ be such that $\|a\|_{A_{\theta}^{\infty}}<1$, and consider $f \in \mathcal{F}^{\infty}(\bar{A})$ such that $f(\theta)=a$ and $\|f\|_{\mathcal{F}^{\infty}(\bar{A})}<1$. Let $\left(K_{n}\right)$ be a sequence of functions in $L_{1}(\mathbb{R})$ with compact support, such that the sequence is an approximation to the identity in $L_{1}(\mathbb{R})$, that is, $\left(K_{n}\right)$ has the following properties:

(a) $\int_{\mathbb{R}} K_{n}(t) d t=1$ for every $n \in \mathbb{N}$.

(b) $\sup _{n}\left\|K_{n}\right\|_{L_{1}(\mathbb{R})}<\infty$.

(c) For every $\delta>0, \lim _{n \rightarrow \infty} \int_{|t| \geq \delta}\left|K_{n}(t)\right| d t=0$.

Assume, moreover, that $\sup _{n}\left\|K_{n}\right\|_{L_{1}(\mathbb{R})}=1$. For each $z \in \overline{\mathbb{S}}$ and $n \in \mathbb{N}$, let

$$
f_{n}(z)=e^{\left(z^{2}-1\right) / n} \int_{\mathbb{R}} K_{n}(t) f(z-i t) d t
$$

Note that there are $M_{1}, M_{2}>0$ such that for every $z \in \overline{\mathbb{S}}$ and $n \in \mathbb{N},\left|e^{\left(z^{2}-1\right) / n}\right| \leq M_{1}$ and $\|f(z)\|_{A_{0}+A_{1}} \leq M_{2}$. Let us show that $f_{n} \in \mathcal{F}(\bar{A})$ for all $n \in \mathbb{N}$ :

(a) $f_{n}: \overline{\mathbb{S}} \longrightarrow A_{0}+A_{1}$ is continuous: the proof is based on properties of convolution and will not be included here.

(b) $f_{n}$ is bounded: Let $z \in \overline{\mathbb{S}}$, we have

$$
\begin{aligned}
\left\|f_{n}(z)\right\|_{A_{0}+A_{1}} & \leq\left|e^{\left(z^{2}-1\right) / n}\right| \int_{\mathbb{R}}\left|K_{n}(t)\right|\|f(z-i t)\|_{A_{0}+A_{1}} d t \\
& \leq M_{1} M_{2}\left\|K_{n}\right\|_{L_{1}(\mathbb{R})} \leq M_{1} M_{2} \sup _{n}\left\|K_{n}\right\|_{L_{1}(\mathbb{R})}=M_{1} M_{2}
\end{aligned}
$$

(c) $f_{n}$ is holomorphic on $\mathbb{S}$ : Let $a^{*} \in\left(A_{0}+A_{1}\right)^{*}$. For all $z \in \mathbb{S}$ we have from Hille's Theorem (Theorem 1.5.5)

$$
a^{*}\left(f_{n}(z)\right)=a^{*}\left(e^{\left(z^{2}-1\right) / n} \int_{\mathbb{R}} K_{n}(t) f(z-i t) d t\right)=e^{\left(z^{2}-1\right) / n} \int_{\mathbb{R}} K_{n}(t) a^{*}(f(z-i t)) d t
$$

Since $a^{*} \circ f$ is holomorphic in the usual sense, it follows that $a^{*} \circ f_{n}$ is holomorphic in the usual sense (integral of holomorphic function is holomorphic). Thus, $f_{n}: \mathbb{S} \longrightarrow A_{0}+A_{1}$ is holomorphic.

(d) For $j=0,1, t \in \mathbb{R}$, we have that $f_{n}(j+i t)=e^{\left((j+i t)^{2}-1\right) / n} \int_{\mathbb{R}} K_{n}(s) f(j+i t-i s) d s \in A_{j}$. Moreover,

$$
\begin{aligned}
\left\|f_{n}(j+i t)\right\|_{A_{j}} & =e^{\left(j^{2}-t^{2}-1\right) / n}\left\|\int_{\mathbb{R}} K_{n}(s) f(j+i t-i s) d s\right\|_{A_{j}} \\
& \leq e^{-t^{2} / n} \int_{\mathbb{R}}\left|K_{n}(s)\right|\|f(j+i(t-s))\|_{A_{j}} \\
& \leq e^{-t^{2} / n}\|f\|_{\mathcal{F}^{\infty}(\bar{A})} \sup _{m}\left\|K_{m}\right\|_{L_{1}(\mathbb{R})} \stackrel{|t| \rightarrow \infty}{\longrightarrow} 0
\end{aligned}
$$


Thus, $f_{n} \in \mathcal{F}(\bar{A}) \subseteq \mathcal{F}^{\infty}(\bar{A})$. For $a_{n} \doteq f_{n}(\theta) \in A_{\theta}$, note that

$$
\left\|a_{n}\right\|_{A_{\theta}}=\inf \left\{\|f\|_{\mathcal{F}(\bar{A})}: f \in \mathcal{F}(\bar{A}), f(\theta)=a_{n}\right\} \leq\left\|f_{n}\right\|_{\mathcal{F}(\bar{A})} \leq 1
$$

Let us show, lastly, that $\left\|a_{n}-a\right\|_{A_{\theta}^{\infty}} \stackrel{n \rightarrow \infty}{\longrightarrow} 0$. From Proposition 3.2.1 we have that $A_{\theta}^{\infty}=B_{z_{0}}^{\infty}=$ $B_{z_{0}}^{1}=A_{\theta}^{1}$. Since $f_{n}, f \in \mathcal{F}^{\infty}(\bar{A})$, we have that $f_{n}, f \in \mathcal{F}_{\theta}^{1}(\bar{A})$ and

$$
\begin{aligned}
\left\|a_{n}-a\right\|_{A_{\theta}^{\infty}} & \leq\left\|f_{n}-f\right\|_{\mathcal{F}^{\infty}(\bar{A})} \leq\left\|f_{n}-f\right\|_{\mathcal{F}_{\theta}^{1}(\bar{A})} \\
& =\int_{\mathbb{R}}\left\|f_{n}(i t)-f(i t)\right\|_{A_{0}} d \mu_{\theta, 0}(t)+\int_{\mathbb{R}}\left\|f_{n}(1+i t)-f(1+i t)\right\|_{A_{0}} d \mu_{\theta, 1}(t)
\end{aligned}
$$

Note moreover that, since $\int_{\mathbb{R}} K_{n}(t) d t=1$, we have

$$
\begin{aligned}
\int_{\mathbb{R}}\left\|f_{n}(i t)-f(i t)\right\|_{A_{0}} d \mu_{\theta, 0}(t) & =\int_{\mathbb{R}}\left\|e^{\left(-t^{2}-1\right) / n} \int_{\mathbb{R}} K_{n}(s) f(i(t-s)) d s-f(i t)\right\|_{A_{0}} d \mu_{\theta, 0}(t) \\
& =\int_{\mathbb{R}}\left\|\int_{\mathbb{R}} K_{n}(s)\left[e^{\left(-t^{2}-1\right) / n} f(i(t-s))-f(i t)\right] d s\right\|_{A_{0}} d \mu_{\theta, 0}(t) \\
& \leq \int_{\mathbb{R}}\left[\int_{\mathbb{R}}\left|K_{n}(s)\right|\left\|e^{\left(-t^{2}-1\right) / n} f(i(t-s))-f(i t)\right\|_{A_{0}} d s\right] d \mu_{\theta, 0}(t) \\
& =\int_{\mathbb{R}}\left|K_{n}(s)\right|\left[\int_{\mathbb{R}}\left\|e^{\left(-t^{2}-1\right) / n} f(i(t-s))-f(i t)\right\|_{A_{0}} d \mu_{\theta, 0}(t)\right] d s
\end{aligned}
$$

Let $F: \mathbb{R} \longrightarrow A_{0}$ be given by $F(t)=f(i t)$. Note that $F$ is Bochner-measurable and

$$
\int_{\mathbb{R}}\|F(t)\|_{A_{0}} d \mu_{\theta, 0}(t)=\int_{\mathbb{R}}\|f(i t)\|_{A_{0}} d \mu_{\theta, 0}(t)<\infty,
$$

i.e., $F \in L_{1}\left(\mathbb{R}, \mu_{\theta, 0}, A_{0}\right)$. Let $\epsilon>0$. From Proposition 1.5.14 there is $\varphi: \mathbb{R} \longrightarrow A_{0}$ such that

$$
\begin{gathered}
\varphi=\sum_{j=1}^{m} \varphi_{j} a_{j}, \quad \text { with } m \in \mathbb{N}, a_{j} \in A_{0}, \varphi_{j} \in C_{c}(\mathbb{R}), \\
\int_{\mathbb{R}}\|F(t)-\varphi(t)\|_{A_{0}} d \mu_{\theta, 0}(t)=\|F-\varphi\|_{L_{1}\left(\mathbb{R}, \mu_{\theta, 0}, A_{0}\right)}<\frac{\epsilon}{5}
\end{gathered}
$$

Thus, we have

$$
\begin{aligned}
\int_{\mathbb{R}}\left\|f_{n}(i t)-f(i t)\right\|_{A_{0}} d \mu_{\theta, 0}(t) & \leq \int_{\mathbb{R}}\left|K_{n}(s)\right|\left[\int_{\mathbb{R}}\left\|e^{\left(-t^{2}-1\right) / n} f(i(t-s))-f(i t)\right\|_{A_{0}} d \mu_{\theta, 0}(t)\right] d s \\
& =\int_{\mathbb{R}}\left|K_{n}(s)\right|\left[\int_{\mathbb{R}}\left\|e^{\left(-t^{2}-1\right) / n} F(t-s)-F(t)\right\|_{A_{0}} d \mu_{\theta, 0}(t)\right] d s \\
& \leq \int_{\mathbb{R}}\left|K_{n}(s)\right|\left[\int_{\mathbb{R}}\left\|e^{\left(-t^{2}-1\right) / n} F(t-s)-e^{\left(-t^{2}-1\right) / n} \varphi(t-s)\right\|_{A_{0}} d \mu_{\theta, 0}(t)\right] d s \\
& +\int_{\mathbb{R}}\left|K_{n}(s)\right|\left[\int_{\mathbb{R}}\left\|e^{\left(-t^{2}-1\right) / n} \varphi(t-s)-\varphi(t-s)\right\|_{A_{0}} d \mu_{\theta, 0}(t)\right] d s \\
& +\int_{\mathbb{R}}\left|K_{n}(s)\right|\left[\int_{\mathbb{R}}\|\varphi(t-s)-\varphi(t)\|_{A_{0}} d \mu_{\theta, 0}(t)\right] d s \\
& +\int_{\mathbb{R}}\left|K_{n}(s)\right|\left[\int_{\mathbb{R}}\|\varphi(t)-F(t)\|_{A_{0}} d \mu_{\theta, 0}(t)\right] d s
\end{aligned}
$$

In what follows, we will present some inequalities that will help us to estimate the inequality 
above. To begin, note that

$$
\begin{aligned}
& \int_{\mathbb{R}}\left|K_{n}(s)\right|\left[\int_{\mathbb{R}}\left\|e^{\left(-t^{2}-1\right) / n} F(t-s)-e^{\left(-t^{2}-1\right) / n} \varphi(t-s)\right\|_{A_{0}} d \mu_{\theta, 0}(t)\right] d s \\
& =\int_{\mathbb{R}}\left|K_{n}(s)\right|\left[\int_{\mathbb{R}}\left|e^{\left(-t^{2}-1\right) / n}\right|\|F(t-s)-\varphi(t-s)\|_{A_{0}} d \mu_{\theta, 0}(t)\right] d s \\
& \leq \int_{\mathbb{R}}\left|K_{n}(s)\right|\left[\int_{\mathbb{R}}\|F(t-s)-\varphi(t-s)\|_{A_{0}} d \mu_{\theta, 0}(t)\right] d s \\
& \leq \sup _{m \in \mathbb{N}}\left\|K_{m}\right\|_{L_{1}(\mathbb{R})}\|F-\varphi\|_{L_{1}\left(\mathbb{R}, \mu_{\theta, 0}, A_{0}\right)}<\frac{\epsilon}{5}
\end{aligned}
$$

We have that each $\varphi_{j}$ is bounded (since it is continuous with compact support), thus, there are $M_{j}>0$ such that $\left|\varphi_{j}(t)\right| \leq M_{j}$ for all $t \in \mathbb{R}$. Hence, for every $t \in \mathbb{R}$

$$
\|\varphi(t)\|_{A_{0}}=\left\|\sum_{j=1}^{m} \varphi_{j}(t) a_{j}\right\|_{A_{0}} \leq \sum_{j=1}^{m}\left|\varphi_{j}(t)\right|\left\|a_{j}\right\|_{A_{0}} \leq \sum_{j=1}^{m} M_{j}\left\|a_{j}\right\|_{A_{0}} \doteq M .
$$

Let $n_{1} \in \mathbb{N}$ be such that $\sup _{t \in \mathbb{R}}\left|e^{\left(-t^{2}-1\right) / n}-1\right|<\frac{\epsilon}{5 M(1-\theta)}$ for all $n \geq n_{1}$. It follows that for all $n \geq n_{1}$ we have

$$
\begin{aligned}
& \int_{\mathbb{R}}\left|K_{n}(s)\right|\left[\int_{\mathbb{R}}\left\|e^{\left(-t^{2}-1\right) / n} \varphi(t-s)-\varphi(t-s)\right\|_{A_{0}} d \mu_{\theta, 0}(t)\right] d s \\
& =\int_{\mathbb{R}}\left|K_{n}(s)\right|\left[\int_{\mathbb{R}}\left|e^{\left(-t^{2}-1\right) / n}-1\right|\|\varphi(t-s)\|_{A_{0}} d \mu_{\theta, 0}(t)\right] d s \\
& \leq \int_{\mathbb{R}}\left|K_{n}(s)\right|\left[\int_{\mathbb{R}} M\left|e^{\left(-t^{2}-1\right) / n}-1\right| d \mu_{\theta, 0}(t)\right] d s \\
& =M \int_{\mathbb{R}}\left|e^{\left(-t^{2}-1\right) / n}-1\right|\left[\int_{\mathbb{R}}\left|K_{n}(s)\right| d s\right] d \mu_{\theta, 0}(t) \\
& \leq M \int_{\mathbb{R}}\left|e^{\left(-t^{2}-1\right) / n}-1\right|\left[\sup _{m}\left\|K_{m}\right\|_{L_{1}(\mathbb{R})}\right] d \mu_{\theta, 0}(t) \\
& =M \int_{\mathbb{R}}\left|e^{\left(-t^{2}-1\right) / n}-1\right| d \mu_{\theta, 0}(t) \leq M \int_{\mathbb{R}} \frac{\epsilon}{5 M(1-\theta)} d \mu_{\theta, 0}(t)=\frac{\epsilon}{5}
\end{aligned}
$$

Now, since $\varphi_{j} \in C_{c}(\mathbb{R}), j=1, \ldots, m$, we have that each $\varphi_{j}$ is uniformly continuous. Thus, let $\delta>0$ be such that $\left|\varphi_{j}(t-s)-\varphi_{j}(t)\right|<\frac{\epsilon}{5 m(1-\theta)\left\|a_{j}\right\|_{A_{0}}}, \forall t \in \mathbb{R}, j=1, \ldots, m$, whenever $|s|<\delta$. Moreover, since $\lim _{n} \int_{|s|>\gamma}\left|K_{n}(s)\right| d s=0$ for all $\gamma>0$, let $n_{2} \in \mathbb{N}$ be such that $\int_{|s|>\delta}\left|K_{n}(s)\right| d s \leq$ 
$\frac{\epsilon}{10 M(1-\theta)}$ for all $n \geq n_{2}$. Then, for all $n \geq n_{2}$,

$$
\begin{aligned}
& \int_{\mathbb{R}}\left|K_{n}(s)\right|\left[\int_{\mathbb{R}}\|\varphi(t-s)-\varphi(t)\|_{A_{0}} d \mu_{\theta, 0}(t)\right] d s \\
& =\int_{|s|<\delta}\left|K_{n}(s)\right|\left[\int_{\mathbb{R}}\|\varphi(t-s)-\varphi(t)\|_{A_{0}} d \mu_{\theta, 0}(t)\right] d s \\
& +\int_{|s| \geq \delta}\left|K_{n}(s)\right|\left[\int_{\mathbb{R}}\|\varphi(t-s)-\varphi(t)\|_{A_{0}} d \mu_{\theta, 0}(t)\right] d s \\
& \leq \int_{|s|<\delta}\left|K_{n}(s)\right|\left[\int_{\mathbb{R}} \sum_{j=1}^{m}\left|\varphi_{j}(t-s)-\varphi_{j}(t)\right|\left\|a_{j}\right\|_{A_{0}} d \mu_{\theta, 0}(t)\right] d s \\
& +\int_{|s| \geq \delta}\left|K_{n}(s)\right|\left[\int_{\mathbb{R}} 2 M d \mu_{\theta, 0}(t)\right] d s \\
& \leq \int_{|s|<\delta}\left|K_{n}(s)\right|\left[\int_{\mathbb{R}} \sum_{j=1}^{m} \frac{\epsilon\left\|a_{j}\right\|_{A_{0}}}{5 M(1-\theta)\left\|a_{j}\right\|_{A_{0}}} d \mu_{\theta, 0}(t)\right] d s \\
& +2 M(1-\theta) \int_{|s| \geq \delta}\left|K_{n}(s)\right| d s \\
& =\frac{\epsilon}{5} \int_{|s|<\delta}\left|K_{n}(s)\right| d s+2 M(1-\theta) \int_{|s| \geq \delta}\left|K_{n}(s)\right| d s \\
& \leq \frac{\epsilon}{5}+2 M(1-\theta) \frac{\epsilon}{10 M(1-\theta)}=\frac{\epsilon}{5}+\frac{\epsilon}{5}
\end{aligned}
$$

Replacing equations (3.3), (3.5), (3.6) and (3.7) on (3.4) we then have, for every $n \geq \max \left\{n_{1}, n_{2}\right\}$,

$$
\int_{\mathbb{R}}\left\|f_{n}(i t)-f(i t)\right\|_{A_{0}} d \mu_{\theta, 0}(t)<\frac{\epsilon}{5}+\frac{\epsilon}{5}+\frac{\epsilon}{5}+\frac{\epsilon}{5}+\frac{\epsilon}{5}=\epsilon .
$$

Similarly, it is shown that $\int_{\mathbb{R}}\left\|f_{n}(1+i t)-f(1+i t)\right\|_{A_{0}} d \mu_{\theta, 1}(t) \longrightarrow 0$ as $n \rightarrow \infty$.

Thus, $\left\|a_{n}-a\right\|_{A_{\theta}^{\infty}} \stackrel{n \rightarrow \infty}{\longrightarrow} 0$, from which follows that $B_{A_{\theta}}$ is dense in $B_{A_{\theta}^{\infty}}$.

From Lemma 1.1.2, we have the desired result.

By imposing certain conditions over the interpolation couple, the infimum is reached in equation (2.8). The uniqueness of the function where the minimum is achieved will allow us to find the uniform homeomorphism between the unit spheres of two interpolation spaces.

The following proposition gives us conditions for the existence and uniqueness of the minimal functions.

Proposition 3.2.3. Suppose that the spaces $A_{0}$ and $A_{1}$ are reflexive. Then we have

(a) For all $a \in S_{A_{\theta}}$ there is $g \in \mathcal{H}^{\infty}(\bar{A})$ such that $g\left(z_{0}\right)=a$ and $\left\|g\left(e^{i t}\right)\right\|_{A_{t}}=1$ for almost every $t \in \mathbb{T}$; so we have $\|a\|_{A_{\theta}}=\|g\|_{\mathcal{H}^{\infty}(\bar{A})}$.

(b) If $A_{0}$ is strictly convex then for all $a \in S_{A_{\theta}}$ and for all $1<p<\infty$ there is a unique $g_{a} \in \mathcal{H}_{z_{0}}^{p}(\bar{A})$ such that $g_{a}\left(z_{0}\right)=a$ and $\left\|g_{a}\right\|_{\mathcal{H}_{z_{0}}^{p}(\bar{A})}=1$.

(c) If $A_{0}$ and $A_{1}$ are uniformly convex then the mapping $\Gamma: S_{A_{\theta}} \longrightarrow \mathcal{H}_{z_{0}}^{p}(\bar{A})$ defined by $\Gamma(a)=g_{a}$ is uniformly continuous $(1<p<\infty)$. 
Proof. (a) Let $a \in S_{A_{\theta}}$ and $1<p<\infty$. Consider the set $F_{a}=\left\{h \in \mathcal{H}_{z_{0}}^{p}(\bar{A}): h\left(z_{0}\right)=a\right\}$. Note that $F_{a}$ is nonempty and convex. Let us show that $F_{a}$ is closed: Consider $h \in \overline{F_{a}}$ and a sequence $\left(h_{n}\right)$ in $F_{a}$ such that $\left\|h_{n}-h\right\|_{\mathcal{H}_{z_{0}}^{p}(\bar{A})} \rightarrow 0$. Note that, for every $f \in \mathcal{H}_{z_{0}}^{p}(\bar{A})$, it follows from Hölder's Inequality (Theorem 1.2.2) that

$$
\begin{aligned}
\left\|f\left(z_{0}\right)\right\|_{A_{0}+A_{1}}^{p} & =\left\|\int_{\mathbb{T}} f\left(e^{i t}\right) d \nu_{z_{0}}(t)\right\|_{A_{0}+A_{1}}^{p} \leq\left(\int_{\mathbb{T}}\left\|f\left(e^{i t}\right)\right\|_{A_{0}+A_{1}} d \nu_{z_{0}}(t)\right)^{p} \\
& \leq\left(\int_{\mathbb{T}}\left\|f\left(e^{i t}\right)\right\|_{A_{t}} d \nu_{z_{0}}(t)\right)^{p} \leq \int_{\mathbb{T}}\left\|f\left(e^{i t}\right)\right\|_{A_{t}}^{p} d \nu_{z_{0}}(t)=\|f\|_{\mathcal{H}_{z_{0}}^{p}(\bar{A})}^{p}
\end{aligned}
$$

Thus,

$$
\left\|a-h\left(z_{0}\right)\right\|_{A_{0}+A_{1}}=\left\|h_{n}\left(z_{0}\right)-h\left(z_{0}\right)\right\|_{A_{0}+A_{1}} \leq\left\|h_{n}-h\right\|_{\mathcal{H}_{z_{0}}^{p}(\bar{A})} \stackrel{n \rightarrow \infty}{\longrightarrow} 0
$$

Therefore, $a=h\left(z_{0}\right)$ and $h \in F_{a}$. Since $F_{a}$ is closed, nonempty and convex, and $\mathcal{H}_{z_{0}}^{p}(\bar{A})$ is reflexive (Proposition 3.1.5), it follows from Proposition 1.1.8 that there is a minimal element $\tilde{g} \in F_{a}$ such that

$$
\|\tilde{g}\|_{\mathcal{H}_{z_{0}}^{p}(\bar{A})}=\inf \left\{\|h\|_{\mathcal{H}_{z_{0}}^{p}(\bar{A})}: h \in F_{a}\right\}=\inf \left\{\|h\|_{\mathcal{H}_{z_{0}}^{p}(\bar{A})}: h \in \mathcal{H}_{z_{0}}^{p}(\bar{A}), h\left(z_{0}\right)=a\right\}=\|a\|_{A_{\theta}}=1 .
$$

As we saw in the proof of Proposition 3.2.1, there is $G \in H^{p}(\mathbb{D})$ such that $\left|G\left(e^{i t}\right)\right|=\left\|\tilde{g}\left(e^{i t}\right)\right\|_{A_{t}}$ for almost every $t \in \mathbb{T}$. Moreover, by putting $g=G\left(z_{0}\right) \frac{\tilde{g}}{G}$ we have that $g \in \mathcal{H}^{\infty}(\bar{A})$ and $g\left(z_{0}\right)=a$. Let us show that $\left\|g\left(e^{i t}\right)\right\|_{A_{t}}=1$ for almost every $t \in \mathbb{T}$. Note that

$$
\begin{aligned}
1 & =\|a\|_{A_{\theta}}=\inf \left\{\|h\|_{\mathcal{H}^{\infty}(\bar{A})}: h \in \mathcal{H}^{\infty}(\bar{A}), h\left(z_{0}\right)=a\right\} \leq\|g\|_{\mathcal{H}^{\infty}(\bar{A})} \\
& =\max _{j=0,1}\left\{\underset{t \in I_{j}}{\operatorname{esssup}}\left\|g\left(e^{i t}\right)\right\|_{A_{t}}\right\}=\max _{j=0,1}\left\{\underset{t \in I_{j}}{\operatorname{esssup}}\left\|G\left(z_{0}\right) \frac{\tilde{g}\left(e^{i t}\right)}{G\left(e^{i t}\right)}\right\|_{A_{t}}\right\}=\left|G\left(z_{0}\right)\right|
\end{aligned}
$$

Thus, $\left|G\left(z_{0}\right)\right| \geq 1$. On the other hand we have

$$
\|g\|_{\mathcal{H}^{\infty}(\bar{A})}^{p}=\left|G\left(z_{0}\right)\right|^{p} \leq \int_{\mathbb{T}}\left|G\left(e^{i t}\right)\right|^{p} d \nu_{z_{0}}(t)=\int_{\mathbb{T}}\left\|\tilde{g}\left(e^{i t}\right)\right\|_{A_{t}}^{p} d \nu_{z_{0}}(t)=\|\tilde{g}\|_{\mathcal{H}_{z_{0}}^{p}(\bar{A})}^{p}=\|a\|_{A_{\theta}}^{p}=1
$$

Therefore, $\left|G\left(z_{0}\right)\right| \leq 1$. Thus, $\left\|g\left(e^{i t}\right)\right\|_{A_{t}}=\left|G\left(z_{0}\right)\right|=1$ for almost every $t \in \mathbb{T}$. In addition, note that $1=\|a\|_{A_{\theta}}=\|g\|_{\mathcal{H}^{\infty}(\bar{A})}$.

(b) Let $a \in S_{A_{\theta}}$ and $g_{a} \doteq g \in \mathcal{H}_{z_{0}}^{p}(\bar{A})$ as found in the previous item. Note that $g_{a}\left(z_{0}\right)=a$ and $\left\|g_{a}\right\|_{\mathcal{H}_{z_{0}}^{p}(\bar{A})}^{p}=\int_{\mathbb{T}}\left\|g_{a}\left(e^{i t}\right)\right\|_{A_{t}}^{p} d \nu_{z_{0}}(t)=1$.

Let us show the uniqueness of $g_{a}$. Suppose there is $h \in \mathcal{H}_{z_{0}}^{p}(\bar{A})$ such that $h\left(z_{0}\right)=a,\|h\|_{\mathcal{H}_{z_{0}}^{p}(\bar{A})}=1$ and $g_{a} \neq h$. Since $A_{0}$ is strictly convex, from Proposition 1.5.12 we have that $L_{p}\left(I_{0}, \nu_{z_{0}}, A_{0}\right)$ is strictly convex. It follows then from Proposition 1.1.10 that

$$
\begin{aligned}
\left\|g_{a}+h\right\|_{\mathcal{H}_{z_{0}}^{p}(\bar{A})} & =\left(\int_{I_{0}}\left\|g_{a}\left(e^{i t}\right)+h\left(e^{i t}\right)\right\|_{A_{0}}^{p} d \nu_{z_{0}}(t)+\int_{I_{1}}\left\|g_{a}\left(e^{i t}\right)+h\left(e^{i t}\right)\right\|_{A_{1}}^{p} d \nu_{z_{0}}(t)\right)^{1 / p} \\
& =\left(\left\|g_{a}+h\right\|_{L_{p}\left(I_{0}, \nu_{z_{0}}, A_{0}\right)}^{p}+\left\|g_{a}+h\right\|_{L_{p}\left(I_{1}, \nu_{z_{0}}, A_{1}\right)}^{p}\right)^{1 / p} \\
& <\left[\left(\left\|g_{a}\right\|_{L_{p}\left(I_{0}, \nu_{z_{0}}, A_{0}\right)}+\|h\|_{L_{p}\left(I_{0}, \nu_{z_{0}}, A_{0}\right)}\right)^{p}+\left(\left\|g_{a}\right\|_{L_{p}\left(I_{1}, \nu_{z_{0}}, A_{1}\right)}+\|h\|_{L_{p}\left(I_{1}, \nu_{z_{0}}, A_{1}\right)}\right)^{p}\right]^{1 / p} .
\end{aligned}
$$


Let $q$ denote the conjugate exponent of $p$. It follows from Hölder's Inequality (Theorem 1.2.2) that for $j=0,1$

$$
\begin{aligned}
\left(\left\|g_{a}\right\|_{L_{p}\left(I_{j}, \nu_{z_{0}}, A_{j}\right)}+\|h\|_{L_{p}\left(I_{j}, \nu_{z_{0}}, A_{j}\right)}\right)^{p} & =2^{\frac{p}{q}}\left(2^{-\frac{1}{q}}\left\|g_{a}\right\|_{L_{p}\left(I_{j}, \nu_{z_{0}}, A_{j}\right)}+2^{-\frac{1}{q}}\|h\|_{L_{p}\left(I_{j}, \nu_{z_{0}}, A_{j}\right)}\right)^{p} \\
& \leq 2^{\frac{p}{q}}\left(\left\|g_{a}\right\|_{L_{p}\left(I_{j}, \nu_{z_{0}}, A_{j}\right)}^{p}+\|h\|_{L_{p}\left(I_{j}, \nu_{z_{0}}, A_{j}\right)}^{p}\right)
\end{aligned}
$$

Thus, replacing (3.9) in (3.8) we have

$$
\begin{aligned}
\left\|g_{a}+h\right\|_{\mathcal{H}_{z_{0}}^{p}(\bar{A})} & <\left[\left(\left\|g_{a}\right\|_{L_{p}\left(I_{0}, \nu_{z_{0}}, A_{0}\right)}+\|h\|_{L_{p}\left(I_{0}, \nu_{z_{0}}, A_{0}\right)}\right)^{p}+\left(\left\|g_{a}\right\|_{L_{p}\left(I_{1}, \nu_{z_{0}}, A_{1}\right)}+\|h\|_{L_{p}\left(I_{1}, \nu_{z_{0}}, A_{1}\right)}\right)^{p}\right]^{1 / p} \\
& \leq 2^{\frac{1}{q}}\left(\left\|g_{a}\right\|_{L_{p}\left(I_{0}, \nu_{z_{0}}, A_{0}\right)}^{p}+\|h\|_{L_{p}\left(I_{0}, \nu_{z_{0}}, A_{0}\right)}^{p}+\left\|g_{a}\right\|_{L_{p}\left(I_{1}, \nu_{z_{0}}, A_{1}\right)}^{p}+\|h\|_{L_{p}\left(I_{1}, \nu_{z_{0}}, A_{1}\right)}^{p}\right)^{\frac{1}{p}} \\
& =2^{\frac{1}{q}}\left(\left\|g_{a}\right\|_{\mathcal{H}_{z_{0}}^{p}(\bar{A})}^{p}+\|h\|_{\mathcal{H}_{z_{0}}^{p}(\bar{A})}^{p}\right)^{\frac{1}{p}}=2^{\frac{1}{q}} 2^{\frac{1}{p}}=2
\end{aligned}
$$

Hence, $\left\|\frac{g_{a}+h}{2}\right\|_{\mathcal{H}_{z_{0}}^{p}(\bar{A})}<1$. However, note that $\frac{g_{a}+h}{2} \in \mathcal{H}_{z_{0}}^{p}(\bar{A})$ and $\left(\frac{g_{a}+h}{2}\right)\left(z_{0}\right)=a$; thus, $\left\|\frac{g_{a}+h}{2}\right\|_{\mathcal{H}_{z_{0}}^{p}(\bar{A})} \geq$ $\|a\|_{A_{\theta}}=1$ and we have a contradiction.

(c) Since $A_{0}$ and $A_{1}$ are uniformly convex spaces, it follows from Proposition 3.1.6 that $\mathcal{H}_{z_{0}}^{p}(\bar{A})$ is uniformly convex. Let $\delta$ denote the modulus of convexity of $\mathcal{H}_{z_{0}}^{p}(\bar{A})$ and consider $a, b \in S_{A_{\theta}}$. Note that

$$
1=\|a\|_{A_{\theta}}=\left\|\frac{a+b+a-b}{2}\right\|_{A_{\theta}} \leq\left\|\frac{a+b}{2}\right\|_{A_{\theta}}+\left\|\frac{a-b}{2}\right\|_{A_{\theta}},
$$

which implies that

$$
\left\|\frac{a-b}{2}\right\|_{A_{\theta}} \geq 1-\left\|\frac{a+b}{2}\right\|_{A_{\theta}}
$$

Moreover, since $\frac{\Gamma(a)+\Gamma(b)}{2} \in \mathcal{H}_{z_{0}}^{p}(\bar{A})$ and $\left(\frac{\Gamma(a)+\Gamma(b)}{2}\right)(\theta)=\frac{a+b}{2}$, we have

$$
\left\|\frac{a+b}{2}\right\|_{A_{\theta}} \leq\left\|\frac{\Gamma(a)+\Gamma(b)}{2}\right\|_{\mathcal{H}_{z_{0}}^{p}(\bar{A})} .
$$

Thus,

$$
\begin{aligned}
\left\|\frac{a-b}{2}\right\|_{A_{\theta}} & \geq 1-\left\|\frac{a+b}{2}\right\|_{A_{\theta}} \geq 1-\left\|\frac{\Gamma(a)+\Gamma(b)}{2}\right\|_{\mathcal{H}_{z_{0}}^{p}(\bar{A})} \\
& \geq 1-\left(1-\delta\left(\|\Gamma(a)-\Gamma(b)\|_{\mathcal{H}_{z_{0}}^{p}(\bar{A})}\right)\right)=\delta\left(\|\Gamma(a)-\Gamma(b)\|_{\mathcal{H}_{z_{0}}^{p}(\bar{A})}\right)
\end{aligned}
$$

Hence, $\Gamma$ is uniformly continuous.

Remark 3.2.4. We saw in the previous section that the spaces $\mathcal{H}_{z_{0}}^{p}(\bar{A})$ and $\mathcal{F}_{\theta}^{p}(\bar{A})$ are isometric. Thus, if $\Psi: \mathcal{H}_{z_{0}}^{p}(\bar{A}) \longrightarrow \mathcal{F}_{\theta}^{p}(\bar{A})$ defines an isometry (and therefore, it is uniformly continuous), then with the notation of the above proposition, we have that $\Psi \circ \Gamma: S_{A_{\theta}} \longrightarrow \mathcal{F}_{\theta}^{p}(\bar{A})$ is uniformly continuous. From now on, to facilitate notation, we will consider $\Gamma: S_{A_{\theta}} \longrightarrow \mathcal{F}_{\theta}^{p}(\bar{A})$, that is, we will omit the isometry $\Psi$. Given $a \in S_{A_{\theta}}, \Gamma(a) \in \mathcal{F}_{\theta}^{p}(\bar{A})$ is what we call minimal function. The 
uniformly continuous function $\Gamma: S_{A_{\theta}} \longrightarrow \mathcal{F}_{\theta}^{p}(\bar{A})$ is what we call minimal lifting map.

Moreover, note that from Proposition 3.2.3, the $g_{a}$ found does not depend on $p$, thus, we can consider $\Gamma: S_{A_{\theta}} \longrightarrow \mathcal{F}^{\infty}(\bar{A})$. Recall that the space $\mathcal{F}^{\infty}(\bar{A})$ is independent of $\theta$.

Gathering all the above propositions, we finally obtain Daher's main result, which gives us a uniform homeomorphism between spheres of interpolation spaces.

Theorem 3.2.5. Let $\left(A_{0}, A_{1}\right)$ be a compatible Banach couple, $0<\theta_{1}, \theta_{2}<1$ with $A_{0} \cap A_{1}$ dense in both $A_{0}$ and $A_{1}$. If $A_{0}$ and $A_{1}$ are uniformly convex, then the mapping $U: S_{A_{\theta_{1}}} \longrightarrow S_{A_{\theta_{2}}}$ given by $U(a)=\Gamma(a)\left(\theta_{2}\right)$ is a uniform homeomorphism.

Proof. Let $a \in S_{A_{\theta_{1}}}$ and consider $f \doteq \Gamma(a) \in \mathcal{F}^{\infty}(\bar{A})$. Note that $\|f\|_{\mathcal{F}^{\infty}(\bar{A})}=1$. Put $b \doteq f\left(\theta_{2}\right)=$ $\Gamma(a)\left(\theta_{2}\right)$. Let us show first that $U$ is well-defined, i.e., $U(a)=b \in S_{A_{\theta_{2}}}$. Note that since $f \in \mathcal{F}^{\infty}(\bar{A})$, we have $f\left(\theta_{2}\right)=b \in A_{\theta_{2}}$; let us show that $\|b\|_{A_{\theta_{2}}}=1$. From the Hahn-Banach Theorem (Corollary 1.1.4) there is $a^{*} \in A_{\theta_{1}}^{*}$ such that $\left\|a^{*}\right\|_{A_{\theta_{1}}^{*}}=1$ and $a^{*} a=1$. From Theorem 2.3.7, we have that $A_{\theta_{1}}^{*}=\left(A_{0}^{*}, A_{1}^{*}\right)_{\theta_{1}}$. Denote $\bar{A}^{*}=\left(A_{0}^{*}, A_{1}^{*}\right)$. Applying Proposition 3.2.3(a) to the element $a^{*} \in S_{A_{\theta_{1}}^{*}}$ there is $f^{*} \in \mathcal{F}_{\theta_{1}}^{2}\left(\bar{A}^{*}\right)$ such that $f^{*}\left(\theta_{1}\right)=a^{*},\left\|f^{*}\right\|_{\mathcal{F}_{\theta_{1}}^{2}\left(\bar{A}^{*}\right)}=1$ and $\left\|f^{*}(j+i t)\right\|_{A_{j}^{*}}=1$ for almost every $t \in \mathbb{R}, j=0,1$. We also know that for every $z \in \mathbb{S}$

$$
f^{\star}(z)=\int_{\mathbb{R}} f^{\star}(i t) d \mu_{z, 0}(t)+\int_{\mathbb{R}} f^{\star}(1+i t) d \mu_{z, 1}(t) .
$$

Thus,

$$
\left\|f^{*}(z)\right\|_{A_{0}^{*}+A_{1}^{*}} \leq \int_{\mathbb{R}}\left\|f^{*}(i t)\right\|_{A_{0}^{*}} d \mu_{z, 0}(t)+\int_{\mathbb{R}}\left\|f^{*}(1+i t)\right\|_{A_{1}^{*}} d \mu_{z, 1}(t)=\int_{\mathbb{R}} d \mu_{z, 0}(t)+\int_{\mathbb{R}} d \mu_{z, 1}(t)=1 .
$$

Let $G: \mathbb{S} \longrightarrow \mathbb{C}$ given by $G(z)=f^{*}(z)(f(z))$. Note that $G$ is holomorphic. Moreover, for every $z \in \mathbb{S}$ we have

$$
|G(z)|=\left|f^{*}(z)(f(z))\right| \leq\left\|f^{*}(z)\right\|_{A_{0}^{*}+A_{1}^{*}}\|f(z)\|_{A_{0}+A_{1}} \leq\left\|f^{*}(z)\right\|_{A_{0}^{*}+A_{1}^{*}}\|f\|_{\mathcal{F}^{\infty}(\bar{A})} \leq 1,
$$

and $\left|G\left(\theta_{1}\right)\right|=\left|f^{*}\left(\theta_{1}\right)\left(f\left(\theta_{1}\right)\right)\right|=a^{*} a=1$. If follows from the Maximum Modulus Principle that $G$ is constant and $G \equiv 1$. In particular, $1=G\left(\theta_{2}\right)=f^{\star}\left(\theta_{2}\right)\left(f\left(\theta_{2}\right)\right)=f^{\star}\left(\theta_{2}\right)(b)$, thus,

$$
1=f^{*}\left(\theta_{2}\right)(b) \leq\left\|f^{*}\left(\theta_{2}\right)\right\|_{A_{0}^{*}+A_{1}^{*}}\|b\|_{A_{\theta_{2}}} \leq\|b\|_{A_{\theta_{2}}} .
$$

On the other hand we have $\|b\|_{A_{\theta_{2}}} \leq\|f\|_{\mathcal{F}^{\infty}(\bar{A})}=1$. Therefore, $\|U(a)\|_{A_{\theta_{2}}}=\|b\|_{A_{\theta_{2}}}=1$.

Let us show that $U$ is uniformly continuous. Let $\epsilon>0$. From Proposition 3.2.3(c) we have that

$$
\Gamma: S_{A_{\theta_{1}}} \longrightarrow \mathcal{F}_{\theta_{1}}^{p}(\bar{A})
$$

$(1<p<\infty)$ is uniformly continuous, thus, there is $\delta>0$ such that for all $a_{1}, a_{2} \in S_{A_{\theta_{1}}}$ with $\left\|a_{1}-a_{2}\right\|_{A_{\theta_{1}}}<\delta$ we have $\left\|\Gamma\left(a_{1}\right)-\Gamma\left(a_{2}\right)\right\|_{\mathcal{F}_{\theta_{1}}^{p}(\bar{A})}<\epsilon$. 
Let us show that there is a constant $C\left(\theta_{1}, \theta_{2}\right)>0$ such that

$$
\left\|\Gamma\left(a_{1}\right)-\Gamma\left(a_{2}\right)\right\|_{\mathcal{F}_{\theta_{2}}^{p}(\bar{A})} \leq C\left(\theta_{1}, \theta_{2}\right)\left\|\Gamma\left(a_{1}\right)-\Gamma\left(a_{2}\right)\right\|_{\mathcal{F}_{\theta_{1}}^{p}(\bar{A})}
$$

Consider $z_{1}=\Lambda\left(\theta_{1}\right) \in \mathbb{R}, z_{2}=\Lambda\left(\theta_{2}\right) \in \mathbb{R}$, and

$$
g=\left(\Gamma\left(a_{1}\right)-\Gamma\left(a_{2}\right)\right) \circ \Lambda^{-1} \in \mathcal{H}_{z_{1}}^{p}(\bar{A}), \mathcal{H}_{z_{2}}^{p}(\bar{A})
$$

We will use the conformal map $\Lambda: \overline{\mathbb{S}} \longrightarrow \overline{\mathbb{D}}$ between the strip and the disk to transfer the calculations to the spaces $\mathcal{H}_{z_{1}}^{p}(\bar{A})$ and $\mathcal{H}_{z_{2}}^{p}(\bar{A})$.

Note that for all $t \in \mathbb{R}$

$$
\frac{1-z_{j}}{1+z_{j}} \leq P_{z_{j}}(t)=\frac{1-z_{j}^{2}}{z_{j}^{2}-2 z_{j} \cos t+1} \leq \frac{1+z_{j}}{1-z_{j}}, j=0,1
$$

Thus,

$$
\frac{1-z_{1}}{1+z_{1}} \frac{1-z_{2}}{1+z_{2}} \leq \frac{P_{z_{2}}(t)}{P_{z_{1}}(t)} \leq \underbrace{\frac{1+z_{1}}{1-z_{1}} \frac{1+z_{2}}{1-z_{2}}}_{\doteq C\left(z_{1}, z_{2}\right)}
$$

Therefore,

$$
\begin{aligned}
\|g\|_{\mathcal{H}_{z_{2}}^{p}(\bar{A})}^{p} & =\int_{\mathbb{T}}\left\|g\left(e^{i t}\right)\right\|_{A_{t}}^{p} d \nu_{z_{2}}(t)=\int_{\mathbb{T}}\left\|g\left(e^{i t}\right)\right\|_{A_{t}}^{p} d P_{z_{2}}(t) d m(t) \\
& \leq C\left(z_{1}, z_{2}\right) \int_{\mathbb{T}}\left\|g\left(e^{i t}\right)\right\|_{A_{t}}^{p} d P_{z_{1}}(t) d m(t)=C\left(z_{1}, z_{2}\right)\|g\|_{\mathcal{H}_{z_{1}}^{p}(\bar{A})}^{p}
\end{aligned}
$$

Returning to the spaces $\mathcal{F}_{\theta_{1}}^{p}(\bar{A})$ and $\mathcal{F}_{\theta_{2}}^{p}(\bar{A})$ through the conformal mapping $\Lambda: \overline{\mathbb{S}} \longrightarrow \overline{\mathbb{D}}$, we have that there is $C\left(\theta_{1}, \theta_{2}\right)>0$ such that

$$
\left\|\Gamma\left(a_{1}\right)-\Gamma\left(a_{2}\right)\right\|_{\mathcal{F}_{\theta_{2}}^{p}(\bar{A})} \leq C\left(\theta_{1}, \theta_{2}\right)\left\|\Gamma\left(a_{1}\right)-\Gamma\left(a_{2}\right)\right\|_{\mathcal{F}_{\theta_{1}}^{p}(\bar{A})}
$$

Hence,

$$
\begin{aligned}
\left\|U\left(a_{1}\right)-U\left(a_{2}\right)\right\|_{A_{\theta_{2}}} & =\left\|\Gamma\left(a_{1}\right)\left(\theta_{2}\right)-\Gamma\left(a_{2}\right)\left(\theta_{2}\right)\right\|_{A_{\theta_{2}}}=\left\|\left(\Gamma\left(a_{1}\right)-\Gamma\left(a_{2}\right)\right)\left(\theta_{2}\right)\right\|_{A_{\theta_{2}}} \\
& =\inf \left\{\|f\|_{\mathcal{F}_{\theta_{2}}^{p}(\bar{A})}: f \in \mathcal{F}_{\theta_{2}}^{p}(\bar{A}), f\left(\theta_{2}\right)=U\left(a_{1}\right)-U\left(a_{2}\right)\right\} \\
& \leq\left\|\Gamma\left(a_{1}\right)-\Gamma\left(a_{2}\right)\right\|_{\mathcal{F}_{\theta_{2}}^{p}(\bar{A})} \leq C\left(\theta_{1}, \theta_{2}\right)\left\|\Gamma\left(a_{1}\right)-\Gamma\left(a_{2}\right)\right\|_{\mathcal{F}_{\theta_{1}}^{p}(\bar{A})}<C\left(\theta_{1}, \theta_{2}\right) \epsilon
\end{aligned}
$$

and we have that $U$ is uniformly continuous.

Let us show finally that $U$ is invertible, where the inverse mapping $V \doteq U^{-1}$ is the analogue application that associates to each $b \in S_{A_{\theta_{2}}}$ the value in $\theta_{1}$ of its minimal representation. Let $b \in S_{A_{\theta_{2}}}$, from Proposition 3.2.3, there is a unique $f_{b} \in \mathcal{F}^{\infty}(\bar{A})$ such that $\left\|f_{b}\right\|_{\mathcal{F}^{\infty}(\bar{A})}=1$ and $f_{b}\left(\theta_{2}\right)=b$. We define $V: S_{A_{\theta_{2}}} \longrightarrow S_{A_{\theta_{1}}}$ by

$$
V(b)=f_{b}\left(\theta_{1}\right)
$$

Note that from what we saw above, $V$ is well-defined and is uniformly continuous. Let us show 
that $U(V(b))=b$. Consider $a \doteq f_{b}\left(\theta_{1}\right) \in S_{A_{\theta_{1}}}$. From Proposition 3.2.3 there is a unique $f_{a} \in \mathcal{F}^{\infty}(\bar{A})$ such that $\left\|f_{a}\right\|_{\mathcal{F}^{\infty}(\bar{A})}=1$ and $f_{a}\left(\theta_{1}\right)=a$. Note that $\Gamma(a)=f_{a}$. It follows from the uniqueness of the minimal function that $f_{a} \equiv f_{b}$. Thus,

$$
U(V(b))=U\left(f_{b}\left(\theta_{1}\right)\right)=U\left(f_{a}\left(\theta_{1}\right)\right)=U(a)=f_{a}\left(\theta_{2}\right)=f_{b}\left(\theta_{2}\right)=b .
$$

Similarly, it is shown that for all $a \in S_{A_{\theta_{1}}}$ we have $V(U(a))=a$.

Therefore, $U$ is a uniform homeomorphism between $S_{A_{\theta_{1}}}$ and $S_{A_{\theta_{2}}}$.

Remark 3.2.6. M. Cwikel and S. Reisner proved in [CR82] that given a compatible Banach couple $\left(A_{0}, A_{1}\right)$ and $0<\theta<1$ with at least one of $A_{0}$ or $A_{1}$ uniformly convex, then $A_{\theta}$ is uniformly convex. It follows from the Reiteration Theorem (Theorem 2.3.8) that $A_{\theta}=\left(A_{0}, A_{\beta}\right)_{\gamma}$, with equal norms, where $0<\beta, \gamma<1$ and such that $\theta=\beta \gamma$. Therefore, in the previous theorem, it is sufficient to assume that $A_{0}$ or $A_{1}$ is uniformly convex.

\subsection{Minimal Functions in $L_{p}$ Spaces}

It is well-know that for $1<p, q<\infty$, the unit spheres of $L_{p}$ and $L_{q}$ are uniformly homeomorphic. An explicit form for a uniform homeomorphism was given by S. Mazur [Maz29], and the following is called the Mazur map:

$$
\begin{aligned}
M: S_{L_{p}} & \longrightarrow S_{L_{q}} \\
f & \longmapsto \operatorname{sgn}(f)|f|^{p / q}
\end{aligned}
$$

The purpose of this section is to obtain an explicit form for the minimal function and minimal lifting map associated with the interpolation space $L_{p}$. We will show that the uniform homeomorphism given by the Daher's Theorem coincides with the Mazur map.

Let $m$ denote the Lebesgue measure on $\Omega=(0,1), \mathcal{M}$ the Borel $\sigma$-algebra on $(0,1)$, and for $1 \leq p \leq \infty$ we put $L_{p}=L_{p}(\Omega, \mathcal{M}, m)$.

Consider $A_{0}=L_{p_{0}}$ and $A_{1}=L_{p_{1}}$, with $1<p_{0}<p_{1}<\infty$. For $0<\theta<1$ we have from Theorem 2.4.1 that

$$
\left(L_{p_{0}}, L_{p_{1}}\right)_{\theta}=L_{p_{\theta}}, \quad \text { with } \frac{1}{p_{\theta}}=\frac{1-\theta}{p_{0}}+\frac{\theta}{p_{1}} .
$$

It follows from Proposition 1.2.6 that $L_{p_{0}} \cap L_{p_{1}}$ is dense in both $L_{p_{0}}$ and $L_{p_{1}}$. From Proposition 1.2.10, we have that $L_{p_{0}}$ and $L_{p_{1}}$ are uniformly convex spaces. Thus, according to Proposition 3.2.3, for each $f \in S_{L_{p_{\theta}}}$ and $1<p<\infty$ there is one and only one $\Gamma(f) \in \mathcal{F}_{\theta}^{p}(\bar{A})$ such that $\Gamma(f)(\theta)=f$ and $\|\Gamma(f)\|_{\mathcal{F}_{\theta}^{p}(\bar{A})}=1$. The function $\Gamma: S_{L_{p_{\theta}}} \longrightarrow \mathcal{F}_{\theta}^{p}(\bar{A})$ is the minimal lifting map associated to the space $L_{p_{\theta}}$ and to the couple $\left(L_{p_{0}}, L_{p_{1}}\right)$. We will show that, for all $z \in \overline{\mathbb{S}}$ and $f \in S_{L_{p_{\theta}}}$,

$$
\Gamma(f)(z)=\operatorname{sgn}(f)|f|^{p_{\theta}\left(\frac{1-z}{p_{0}}+\frac{z}{p_{1}}\right)} .
$$

Note that, since $1<p_{0}<p_{1}<+\infty$, we can consider $\epsilon>0$ such that $p_{0}-\epsilon>1$ and $p_{1}+\epsilon<+\infty$. 
Let $p_{0}^{\prime}=p_{0}-\epsilon$ and $p_{1}^{\prime}=p_{1}+\epsilon$; with this, we have that $L_{p_{0}^{\prime}}$ and $L_{p_{1}^{\prime}}$ are Banach spaces. Consider $\mathcal{R}=\{z \in \mathbb{C}:-\epsilon<\mathfrak{R} z<1+\epsilon\}$ and for each $f \in S_{L_{p_{\theta}}}$ and $z \in \overline{\mathcal{R}}$ let

$$
\phi(f)(z)=\operatorname{sgn}(f)|f|^{p_{\theta}\left(\frac{1-z}{p_{0}}+\frac{z}{p_{1}}\right)} .
$$

If $z=r+i t$, note that we have

$$
|\phi(f)(z)|=|f|^{p_{\theta}\left(\frac{1-r}{p_{0}}+\frac{r}{p_{1}}\right)}
$$

and then, the function $\phi(f): \overline{\mathcal{R}} \longrightarrow L_{p_{0}^{\prime}}+L_{p_{1}^{\prime}}$ is well-defined.

Lemma 3.3.1. If $f \in L_{p_{\theta}}$ is a simple function, then $\phi(f)$ is holomorphic on $\mathcal{R}$.

Proof. Consider $f=\sum_{j=1}^{n} \alpha_{j} \chi_{E_{j}}$ written in the canonical representation (with $\alpha_{j} \in \mathbb{C}$ and $E_{i} \cap E_{j}=\varnothing$ if $i \neq j)$. Let $\varphi \in\left(L_{p_{0}^{\prime}}+L_{p_{1}^{\prime}}\right)^{*}$, then we have

$$
\begin{aligned}
\varphi(\phi(f)(z)) & =\varphi\left(\operatorname{sgn}(f)\left|\sum_{j=1}^{n} \alpha_{j} \chi_{E_{j}}\right|^{p_{\theta}\left(\frac{1-z}{p_{0}}+\frac{z}{p_{1}}\right)}\right)=\varphi\left(\operatorname{sgn}(f) \sum_{j=1}^{n}\left|\alpha_{j}\right|^{p_{\theta}\left(\frac{1-z}{p_{0}}+\frac{z}{p_{1}}\right)} \chi_{E_{j}}\right) \\
& =\sum_{j=1}^{n}\left|\alpha_{j}\right|^{p_{\theta}\left(\frac{1-z}{p_{0}}+\frac{z}{p_{1}}\right)} \varphi\left(\operatorname{sgn}(f) \chi_{E_{j}}\right)
\end{aligned}
$$

which is holomorphic, and the result follows from Theorem 1.4.8.

By the previous Lemma and Remark 1.5.9 it follows that, if $f$ is a simple function,

$$
\phi(f)(z)=\int_{\mathbb{R}} \phi(f)(i t) d \mu_{z, 0}(t)+\int_{\mathbb{R}} \phi(f)(1+i t) d \mu_{z, 1}(t), \forall z \in \mathbb{S} .
$$

From now on, we will consider $\phi(f)$ as the restriction to the strip $\overline{\mathbb{S}}$, that is, $\phi(f): \overline{\mathbb{S}} \longrightarrow L_{p_{0}}+L_{p_{1}}$.

Lemma 3.3.2. If $f \in S_{L_{p_{\theta}}}$ is a simple function then the functions $t \longmapsto \phi(f)(j+i t), j=0,1$ are Bochner measurable from $\mathbb{R}$ into $L_{p_{j}}$.

Proof. Note that $\phi(f)(j+i t) \in L_{p_{j}}$ for $j=0,1$ : Indeed, we have

$$
\begin{aligned}
\|\phi(f)(i t)\|_{L_{p_{0}}} & =\left\|\operatorname{sgn}(f)|f|^{p_{\theta}\left(\frac{1-i t}{p_{0}}+\frac{i t}{p_{1}}\right)}\right\|_{L_{p_{0}}}=\left(\int|f|^{\left.p_{\theta}\left(\frac{1-i t}{p_{0}}+\frac{i t}{p_{1}}\right)\right|^{p_{0}}}\right)^{1 / p_{0}} \\
& =\left(\int|f|^{p_{\theta}}\right)^{1 / p_{0}}=\|f\|_{L_{p_{\theta}}}^{p_{\theta / p_{0}}}=1 \\
\|\phi(f)(1+i t)\|_{L_{p_{1}}} & =\left\|\operatorname{sgn}(f)|f|^{p_{\theta}\left(\frac{1-(1+i t)}{p_{0}}+\frac{1+i t}{p_{1}}\right)}\right\|_{L_{p_{1}}}=\left(\left.\left.\int|| f\right|^{p_{\theta}\left(\frac{1-(1+i t)}{p_{0}}+\frac{1+i t}{p_{1}}\right)}\right|^{p_{1}}\right)^{1 / p_{1}} \\
& =\left(\int|f|^{p_{\theta}}\right)^{1 / p_{1}}=\|f\|_{L_{p_{\theta}}}^{p_{\theta / p_{1}}}=1
\end{aligned}
$$

The last equality in the two equations above follows from the fact that $f \in S_{L_{p_{\theta}}}$. Note that 
$\|\phi(f)(i t)\|_{L_{p_{0}}}$ and $\|\phi(f)(1+i t)\|_{L_{p_{1}}}$ no longer depend on $t$.

Now consider $f=\sum_{k=1}^{n} \alpha_{k} \chi_{E_{k}}$ written in the canonical representation. We have for $j=0,1$

$$
\phi(f)(j+i t)=\sum_{k=1}^{n} \underbrace{\left|\alpha_{k}\right|^{p_{\theta}\left(\frac{1-(j+i t)}{p_{0}}+\frac{j+i t}{p_{1}}\right)}}_{\text {continuous in t }} \underbrace{\operatorname{sgn}(f) \chi_{E_{k}}}_{\in L_{p_{j}}},
$$

therefore, we have the result.

Note also that

$$
\begin{aligned}
\|\phi(f)\|_{\mathcal{F}_{\theta}^{p}(\bar{A})}^{p} & =\int_{\mathbb{R}}\|\phi(f)(i t)\|_{L_{p_{0}}}^{p} d \mu_{\theta, 0}(t)+\int_{\mathbb{R}}\|\phi(f)(1+i t)\|_{L_{p_{1}}}^{p} d \mu_{\theta, 1}(t) \\
& =\|f\|_{L_{p_{\theta}}}^{p p_{\theta / p_{0}}} \int_{\mathbb{R}} d \mu_{\theta, 0}+\|f\|_{L_{p_{\theta}}}^{p p_{\theta / p_{1}}} \int_{\mathbb{R}} d \mu_{\theta, 1}(t)=(1-\theta)+\theta=1
\end{aligned}
$$

Therefore, we have that if $f \in S_{L_{p_{\theta}}}$ is a simple function, then $\phi(f) \in \mathcal{F}_{\theta}^{p}(\bar{A})$. Note also that

$$
\phi(f)(\theta)=\operatorname{sgn}(f)|f|^{p_{\theta}\left(\frac{1-\theta}{p_{0}}+\frac{\theta}{p_{1}}\right)}=f
$$

and $\|\phi\|_{\mathcal{F}_{\theta}^{p}(\bar{A})}=1$, that is, $\phi(f)$ satisfy the thesis from Proposition 3.2.3(b), so we have that

$$
\Gamma(f)=\phi(f) \text {, if } f \text { is a simple function. }
$$

Finally, let us show that the result is valid for any $f \in S_{L_{p_{\theta}}}$. Since the simple functions are dense in $L_{p_{\theta}}$, let $\left(f_{n}\right)$ be a sequence of simple functions in $S_{L_{p_{\theta}}}$ such that $\left\|f_{n}-f\right\|_{L_{p_{\theta}}} \rightarrow 0$ as $n \rightarrow \infty$, that is,

$$
\left\|f_{n}-f\right\|_{L_{p_{\theta}}}^{p_{\theta}}=\int\left|f_{n}(s)-f(s)\right|^{p_{\theta}} d m(s) \longrightarrow 0
$$

which implies that $f_{n}(s) \longrightarrow f(s)$ for almost every $s \in(0,1)$.

We have to show that $\left\|\phi\left(f_{n}\right)-\phi(f)\right\|_{\mathcal{F}_{\theta}^{p}(\bar{A})} \longrightarrow 0$. Since $\Gamma: S_{L_{p_{\theta}}} \longrightarrow \mathcal{F}_{\theta_{1}}^{p}(\bar{A})$ is continuous, we have that $\phi\left(f_{n}\right)=\Gamma\left(f_{n}\right) \longrightarrow \Gamma(f)$ in $\mathcal{F}_{\theta}^{p}(\bar{A})$, therefore $\phi\left(f_{n}\right)(j+i t)(s) \rightarrow \Gamma(f)(j+i t)(s)$, for almost every $t \epsilon$ $\mathbb{R}$ and for almost every $s \in(0,1)$. Since

$$
\phi\left(f_{n}\right)(j+i t)(s)=\operatorname{sgn}\left(f_{n}(s)\right)\left|f_{n}(s)\right|^{p_{\theta}\left(\frac{1-(j+i t)}{p_{0}}+\frac{j+i t}{p_{1}}\right)} \longrightarrow \underbrace{\operatorname{sgn}(f(s))|f(s)|^{p_{\theta}\left(\frac{1-(j+i t)}{p_{0}}+\frac{j+i t}{p_{1}}\right)}}_{\phi(f)(j+i t)(s)}
$$

we have that $\Gamma(f)(j+i t)(s)=\phi(f)(j+i t)(s)$ for almost every $t \in \mathbb{R}$ and for almost every $s \in(0,1)$, which implies that $\left\|\phi\left(f_{n}\right)-\phi(f)\right\|_{\mathcal{F}_{\theta}^{p}(\bar{A})} \longrightarrow 0$.

Therefore, the minimal lifting map $\Gamma: S_{L_{p_{\theta}}} \longrightarrow \mathcal{F}_{\theta}^{p}(\bar{A})$ is given by

$$
\Gamma(f)(z)=\operatorname{sgn}(f)|f|^{p_{\theta}\left(\frac{1-z}{p_{0}}+\frac{z}{p_{1}}\right)},
$$

for every $f \in S_{L_{p_{\theta}}}$ and $z \in \overline{\mathbb{S}}$. 
Furthermore, according to Theorem 3.2.5 it follows that for $0<\theta_{1}, \theta_{2}<1$, the mapping

$$
\begin{aligned}
U: S_{L_{p_{\theta_{1}}}} & \longrightarrow S_{L_{p_{\theta_{2}}}} \\
f & \longmapsto \operatorname{sgn}(f)|f|^{\frac{p_{\theta_{1}}}{p_{\theta_{2}}}}
\end{aligned}
$$

defines a uniform homemomorphism between the unit spheres of the interpolation spaces $L_{p_{\theta_{1}}}$ and $L_{p_{\theta_{2}}}$. Note that $U$ is exactly the Mazur Map.

\subsection{Minimal Functions in Weighted $L_{p}$ Spaces}

In this section, we want to give an explicit form for the minimal function and the minimal lifting map in the case of weighted $L_{p}$ spaces. Daher's Theorem requires certain hypothesis for the interpolation couple, such as the density of the intersection in each space of the interpolation couple; with that in mind, we will assume certain hypotheses over the weights that we will consider.

Let $m$ denote the Lebesgue measure in $\Omega=(0,1), \mathcal{M}$ the Borel $\sigma$-algebra on $(0,1)$, and consider the measure space $(\Omega, \mathcal{M}, m)$. For $1<p<\infty$, consider $L_{p}=L_{p}(\Omega, \mathcal{M}, m)$. Let $\epsilon>0$ and $w_{0}, w_{1}$ : $(0,1) \longrightarrow(0, \infty)$ two measurable functions such that

$$
w_{0}^{1+\epsilon} w_{1}^{-\epsilon}, w_{0}^{-\epsilon} w_{1}^{1+\epsilon} \in L_{1}
$$

Let $1<q<\infty$ and $0<\theta<1$. From Theorem 2.5.1 we have, with equal norms,

$$
\left(L_{q}\left(w_{0}\right), L_{q}\left(w_{1}\right)\right)_{\theta}=L_{q}(w)
$$

where $w=w_{0}^{1-\theta} w_{1}^{\theta}$.

Let us show that the spaces $A_{0}=L_{q}\left(w_{0}\right)$ and $A_{1}=L_{q}\left(w_{1}\right)$ satisfy the hypotheses of Proposition 3.2 .3 .

Lemma 3.4.1. For all $s \in(0,1)$, we have $w_{0}(s)+w_{1}(s) \leq w_{0}^{1+\epsilon}(s) w_{1}^{-\epsilon}(s)+w_{0}^{-\epsilon}(s) w_{1}^{1+\epsilon}(s)$.

Proof. Let $f(t)=t^{1+\epsilon}-(1+\epsilon) t, t \geq 0$. Note that $f^{\prime}(t)=(1+\epsilon)\left(t^{\epsilon}-1\right)=0$ if and only if $t=1$. If $t \leq 1, f^{\prime}(t)<0$, thus $f$ is not increasing, and if $t>1, f$ is increasing. Therefore, $t=1$ is a (global) minimum point of $f$, from which follows that $t^{1+\epsilon}-(1+\epsilon) t=f(t) \geq f(1)=-\epsilon, \forall t \geq 0$. Putting $t=\frac{w_{0}}{w_{1}}$, we have $w_{0}^{1+\epsilon} w_{1}^{-\epsilon} \geq(1+\epsilon) w_{0}-\epsilon w_{1}$. Similarly, we obtain $w_{0}^{-\epsilon} w_{1}^{1+\epsilon} \geq-\epsilon w_{0}+(1+\epsilon) w_{1}$. Thus, $w_{0}^{1+\epsilon} w_{1}^{-\epsilon}+w_{0}^{-\epsilon} w_{1}^{1+\epsilon} \geq w_{0}+w_{1}$.

Lemma 3.4.2. $L_{q}\left(w_{0}\right) \cap L_{q}\left(w_{1}\right)$ is dense in both $L_{q}\left(w_{0}\right)$ and $L_{q}\left(w_{1}\right)$.

Proof. Since we are assuming $w_{0}^{1+\epsilon} w_{1}^{-\epsilon}, w_{0}^{-\epsilon} w_{1}^{1+\epsilon} \in L_{1}$, it follows from the previous lemma that $w_{0}, w_{1} \in L_{1}$. Thus, the result follows from Proposition 1.2.6.

From Proposition 1.2.10, we have that $L_{q}\left(w_{0}\right)$ and $L_{q}\left(w_{1}\right)$ are uniformly convex spaces. Thus, according to Proposition 3.2.3, for each $f \in S_{L_{q}(w)}$ and $1<p<\infty$ there is one and only one 
$\Gamma(f) \in \mathcal{F}_{\theta}^{p}(\bar{A})$ such that $\Gamma(f)(\theta)=f$ and $\|\Gamma(f)\|_{\mathcal{F}_{\theta}^{p}(\bar{A})}=1$. We will show that the minimal lifting map $\Gamma: S_{L_{q}(w)} \longrightarrow \mathcal{F}_{\theta}^{p}(\bar{A})$ is given by

$$
\Gamma(f)(z)=f w_{0}^{\frac{z-\theta}{q}} w_{1}^{\frac{\theta-z}{q}}, f \in S_{L_{q}(w)}, z \in \overline{\mathbb{S}} .
$$

Consider $\mathcal{S}=\{z \in \mathbb{C}:-\epsilon<\mathfrak{R} z<1+\epsilon\}$. For each $f \in S_{L_{q}(w)}$ and $z \in \overline{\mathcal{S}}$, define

$$
\Phi(f)(z)=f w_{0}^{\frac{z-\theta}{q}} w_{1}^{\frac{\theta-z}{q}}
$$

We want to show initially that $\Phi(f) \uparrow_{\overline{\mathbb{S}}} \in \mathcal{F}_{\theta}^{p}(\bar{A})$. With this in mind, we need $\Phi(f)$ to be holomorphic on $\mathbb{S}$. The idea is to show that it is holomorphic in a slightly larger strip $\mathcal{S}$, so that Cauchy's Integral Formula and the measurability at the boundary are automatically satisfied (see Remark 1.5.9).

Note that, for $z=r+i t \in \mathcal{S}$, we have

$$
\int_{0}^{1}\left|f w_{0}^{\frac{z-\theta}{q}} w_{1}^{\frac{\theta-z}{q}}\right|^{q} w_{0}^{1-r} w_{1}^{r} d m=\int_{0}^{1}\left|f w_{0}^{r-\theta} w_{1}^{\theta-r}\right|^{q} w_{0}^{1-r} w_{1}^{r} d m=\int_{0}^{1}|f|^{q} w_{0}^{1-\theta} w_{1}^{\theta} d m
$$

Thus,

$$
\Phi(f)(r+i t) \in L_{q}\left(w_{0}^{1-r} w_{1}^{r}\right)
$$

For $r=-\epsilon, \Phi(f)(-\epsilon+i t) \epsilon L_{q}\left(w_{0}^{1+\epsilon} w_{1}^{-\epsilon}\right)$ and for $r=1+\epsilon, \Phi(f)(1+\epsilon+i t) \epsilon L_{q}\left(w_{0}^{-\epsilon} w_{1}^{1+\epsilon}\right)$. Therefore,

$$
\Phi(f): \mathcal{S} \longrightarrow L_{q}\left(w_{0}^{1+\epsilon} w_{1}^{-\epsilon}\right)+L_{q}\left(w_{0}^{-\epsilon} w_{1}^{1+\epsilon}\right)
$$

is well-defined for each $f \in S_{L_{q}(w)}$.

Since we are assuming $w_{0}^{1+\epsilon} w_{1}^{-\epsilon}, w_{0}^{-\epsilon} w_{1}^{1+\epsilon} \epsilon L_{1}$, we have that $L_{q}\left(w_{0}^{1+\epsilon} w_{1}^{-\epsilon}\right) \cap L_{q}\left(w_{0}^{-\epsilon} w_{1}^{1+\epsilon}\right)$ is dense in both $L_{q}\left(w_{0}^{1+\epsilon} w_{1}^{-\epsilon}\right)$ and $L_{q}\left(w_{0}^{-\epsilon} w_{1}^{1+\epsilon}\right)$. Thus, from Theorem 2.2.8, it follows that

$$
\left(L_{q}\left(w_{0}^{1+\epsilon} w_{1}^{-\epsilon}\right)+L_{q}\left(w_{0}^{-\epsilon} w_{1}^{1+\epsilon}\right)\right)^{*}=\left(L_{q}\left(w_{0}^{1+\epsilon} w_{1}^{-\epsilon}\right)\right)^{*} \cap\left(L_{q}\left(w_{0}^{-\epsilon} w_{1}^{1+\epsilon}\right)\right)^{*} .
$$

Lemma 3.4.3. For each $f \in S_{L_{q}(w)}, \Phi(f)$ is holomorphic on $\mathcal{S}$.

Proof. Let us put $B_{0}=L_{q}\left(w_{0}^{1+\epsilon} w_{1}^{-\epsilon}\right)$ and $B_{1}=L_{q}\left(w_{0}^{-\epsilon} w_{1}^{1+\epsilon}\right)$, and let $x^{*} \epsilon\left(B_{0}+B_{1}\right)^{*}=B_{0}^{*} \cap B_{1}^{*}$. We will show that $x^{*} \circ \Phi(f)$ is holomorphic in $\mathcal{S}$ (from which follows that $\Phi(f)$ is holomorphic).

Note that $\nu_{0}=w_{0}^{1+\epsilon} w_{1}^{-\epsilon} d m$ and $\nu_{1}=w_{0}^{-\epsilon} w_{1}^{1+\epsilon} d m$ are positive $\sigma$-finite measures. Thus, since $x^{*} \epsilon B_{j}^{*}$, $j=0,1$, it follows from the Radon-Nikodým Theorem (Theorem 1.2.5) that there is $g_{j} \in B_{j}^{*}$ such that

$$
\begin{aligned}
& x^{*}(h)=\int h(s) g_{0}(s) w_{0}^{1+\epsilon}(s) w_{1}^{-\epsilon}(s) d m(s), \forall h \in B_{0} \\
& x^{*}(h)=\int h(s) g_{1}(s) w_{0}^{-\epsilon}(s) w_{1}^{1+\epsilon}(s) d m(s), \quad \forall h \in B_{1}
\end{aligned}
$$

Let us see that for each $z \in \overline{\mathcal{S}}$ we can write

$$
\Phi(f)(z)=h_{0}(z)+h_{1}(z) \in B_{0}+B_{1}
$$


with $h_{0}(z)=f w_{0}^{\frac{z-\theta}{q}} w_{1}^{\frac{\theta-z}{q}} \chi_{E} \in B_{0}, h_{1}(z)=f w_{0}^{\frac{z-\theta}{q}} w_{1}^{\frac{\theta-z}{q}} \chi_{E^{c}} \in B_{1}$, where $E=\left\{s \in(0,1): 0<\frac{w_{0}(s)}{w_{1}(s)} \leq 1\right\}$ and $E^{c}=\left\{s \in(0,1): \frac{w_{0}(s)}{w_{1}(s)}>1\right\}$. Indeed, for $z=r+i t \in \overline{\mathcal{S}}$, we have

$$
\begin{aligned}
\int\left|h_{0}(z)\right|^{q} w_{0}^{1+\epsilon} w_{1}^{-\epsilon} d m & =\int_{E}|f|^{q} w_{0}^{r-\theta} w_{1}^{\theta-r} w_{0}^{1+\epsilon} w_{1}^{-\epsilon} d m=\int_{E}|f|^{q}\left(w_{0}^{1-\theta} w_{1}^{\theta}\right)\left(\frac{w_{0}}{w_{1}}\right)^{\epsilon+r} d m \\
& \leq \int_{E}|f|^{q}\left(w_{0}^{1-\theta} w_{1}^{\theta}\right) d m \leq\|f\|_{L_{q}(w)}^{q}=1 \\
\int\left|h_{1}(z)\right|^{q} w_{0}^{-\epsilon} w_{1}^{1+\epsilon} d m & =\int_{E^{c}}|f|^{q} w_{0}^{r-\theta} w_{1}^{\theta-r} w_{0}^{-\epsilon} w_{1}^{1+\epsilon} d m=\int_{E^{c}}|f|^{q}\left(w_{0}^{1-\theta} w_{1}^{\theta}\right)\left(\frac{w_{1}}{w_{0}}\right)^{1+\epsilon-r} d m \\
\leq & \int_{E^{c}}|f|^{q}\left(w_{0}^{1-\theta} w_{1}^{\theta}\right) d m \leq\|f\|_{L_{q}(w)}^{q}=1
\end{aligned}
$$

Thus, $h_{0}(z)=f w_{0}^{\frac{z-\theta}{q}} w_{1}^{\frac{\theta-z}{q}} \chi_{E} \in B_{0}$ and $h_{1}(z)=f w_{0}^{\frac{z-\theta}{q}} w_{1}^{\frac{\theta-z}{q}} \chi_{E^{c}} \in B_{1}$. Therefore, for each $z \in \overline{\mathcal{S}}$ we have

$$
\begin{aligned}
x^{*}(\Phi(f)(z)) & =x^{*}\left(h_{0}(z)+h_{1}(z)\right)=x^{*}\left(h_{0}(z)\right)+x^{*}\left(h_{1}(z)\right) \\
& =\int_{0}^{1} h_{0}(z)(s) g_{0}(s)\left(w_{0}^{1+\epsilon} w_{1}^{-\epsilon}\right)(s) d m(s)+\int_{0}^{1} h_{1}(z)(s) g_{1}(s)\left(w_{0}^{-\epsilon} w_{1}^{1+\epsilon}\right)(s) d m(s) \\
& =\int_{E} f(s) w_{0}^{\frac{z-\theta}{q}}(s) w_{1}^{\frac{\theta-z}{q}}(s) g_{0}(s) w_{0}^{1+\epsilon}(s) w_{1}^{-\epsilon}(s) d m(s) \\
& +\int_{E^{c}} f(s) w_{0}^{\frac{z-\theta}{q}}(s) w_{1}^{\frac{\theta-z}{q}}(s) g_{1}(s) w_{0}^{-\epsilon}(s) w_{1}^{1+\epsilon}(s) d m(s)
\end{aligned}
$$

Note that $x^{*}(\Phi(f)(z))$ is continuous on $\mathcal{S}$. Indeed, let $z \in \mathcal{S}$ and $\left(z_{n}\right)$ be a sequence in $\mathcal{S}$ such that $z_{n} \rightarrow z$. Define, for every $s \in E$

$$
\begin{gathered}
F_{n}(s)=f(s) w_{0}^{\frac{z_{n}-\theta}{q}}(s) w_{1}^{\frac{\theta-z_{n}}{q}}(s) g_{0}(s) w_{0}^{1+\epsilon}(s) w_{1}^{-\epsilon}(s) \\
F(s)=f(s) w_{0}^{\frac{z-\theta}{q}}(s) w_{1}^{\frac{\theta-z}{q}}(s) g_{0}(s) w_{0}^{1+\epsilon}(s) w_{1}^{-\epsilon}(s)
\end{gathered}
$$

Note that for fixed $s \in E$, we have that $F_{n}(s) \rightarrow F(s)$ (since $F(s)$ is a continuous function in $z$ ). Moreover, if $z_{n}=r_{n}+i t_{n}$, since $-\epsilon<r_{n}<1+\epsilon$ and $\frac{w_{0}}{w_{1}} \leq 1$ in $E$,

$$
\left|F_{n}(s)\right|=\left[\left|f g_{0}\right| w_{0}^{1+\epsilon} w_{1}^{-\epsilon} w_{0}^{\frac{r_{n}-\theta}{q}} w_{1}^{\frac{\theta-r_{n}}{q}}\right](s) \leq\left[\left|f g_{0}\right|\left(\frac{w_{0}}{w_{1}}\right)^{\frac{-\epsilon-\theta}{q}} w_{0}^{1+\epsilon} w_{1}^{-\epsilon}\right](s)
$$

Note also that from Hölder's Inequality (Theorem 1.2.2) we have

$$
\begin{aligned}
\int_{E}\left|f g_{0}\right|\left(\frac{w_{0}}{w_{1}}\right)^{\frac{-\epsilon-\theta}{q}} w_{0}^{1+\epsilon} w_{1}^{-\epsilon} d m & \leq\left[\int_{E}|f|^{q}\left(\frac{w_{0}}{w_{1}}\right)^{-\epsilon-\theta} w_{0}^{1+\epsilon} w_{1}^{-\epsilon} d m\right]^{\frac{1}{q}}\left[\int_{E}\left|g_{0}\right|^{q^{\prime}} w_{0}^{1+\epsilon} w_{1}^{-\epsilon} d m\right]^{\frac{1}{q^{\prime}}} \\
& =\left[\int_{E}|f|^{q} w_{0}^{1-\theta} w_{1}^{\theta} d m\right]^{\frac{1}{q}}\left[\int_{E}\left|g_{0}\right|^{q^{\prime}} w_{0}^{1+\epsilon} w_{1}^{-\epsilon} d m\right]^{\frac{1}{q^{\prime}}}
\end{aligned}
$$

And since $f \in L_{q}\left(w_{0}^{1-\theta} w_{1}^{\theta}\right)$ and $g_{0} \in B_{0}^{*}=L_{q^{\prime}}\left(w_{0}^{1+\epsilon} w_{1}^{-\epsilon}\right)$, where $q^{\prime}$ is the exponent conjugate to $q$, it follows that $\left|f g_{0}\right|\left(\frac{w_{0}}{w_{1}}\right)^{\frac{-\epsilon-\theta}{q}} w_{0}^{1+\epsilon} w_{1}^{-\epsilon} \in L_{1}\left(E, \mathcal{M} \uparrow_{E}, m\right)$. Thus, from the Lebesgue's Dominated 
Convergence Theorem (Theorem 1.2.1), since $z_{n} \rightarrow z$, we have

$$
\begin{aligned}
x^{*}\left(h_{0}\left(z_{n}\right)\right) & =\int_{E} f(s) w_{0}^{\frac{z_{n}-\theta}{q}}(s) w_{1}^{\frac{\theta-z_{n}}{q}}(s) g_{0}(s) w_{0}^{1+\epsilon}(s) w_{1}^{-\epsilon}(s) d m \longrightarrow \\
& \longrightarrow \int_{E} f(s) w_{0}^{\frac{z-\theta}{q}}(s) w_{1}^{\frac{\theta-z}{q}}(s) g_{0}(s) w_{0}^{1+\epsilon}(s) w_{1}^{-\epsilon}(s) d m=x^{*}\left(h_{0}(z)\right)
\end{aligned}
$$

Similarly, it is shown that $x^{*}\left(h_{1}\left(z_{n}\right)\right) \rightarrow x^{*}\left(h_{1}(z)\right)$. Therefore, when $z_{n} \rightarrow z$

$$
x^{*}\left(\Phi(f)\left(z_{n}\right)\right)=x^{*}\left(h_{0}\left(z_{n}\right)\right)+x^{*}\left(h_{1}\left(z_{n}\right)\right) \longrightarrow x^{*}\left(h_{0}(z)\right)+x^{*}\left(h_{1}(z)\right)=x^{*}(\Phi(f)(z))
$$

and $x^{*}(\Phi(f)(z))$ is continuous over $\mathcal{S}$.

Now, let $\gamma$ be a closed piecewise $C^{1}$ curve in $\mathcal{S}$. We have

$$
\begin{aligned}
\int_{\gamma} x^{*}(\Phi(f)(z)) d z & =\int_{\gamma}\left[\int_{E} f(s) w_{0}^{\frac{z-\theta}{q}}(s) w_{1}^{\frac{\theta-z}{q}}(s) g_{0}(s) w_{0}^{1+\epsilon}(s) w_{1}^{-\epsilon}(s) d m(s)\right] d z \\
& +\int_{\gamma}\left[\int_{E^{c}} f(s) w_{0}^{\frac{z-\theta}{q}}(s) w_{1}^{\frac{\theta-z}{q}}(s) g_{1}(s) w_{0}^{-\epsilon}(s) w_{1}^{1+\epsilon}(s) d m(s)\right] d z \\
& =\int_{E} f(s) g_{0}(s) w_{0}^{1+\epsilon}(s) w_{1}^{-\epsilon}(s)\left[\int_{\gamma} w_{0}^{\frac{z-\theta}{q}}(s) w_{1}^{\frac{\theta-z}{q}}(s) d z\right] d m(s) \\
& +\int_{E^{c}} f(s) g_{1}(s) w_{0}^{-\epsilon}(s) w_{1}^{1+\epsilon}(s)\left[\int_{\gamma} w_{0}^{\frac{z-\theta}{q}}(s) w_{1}^{\frac{\theta-z}{q}}(s) d z\right] d m(s)
\end{aligned}
$$

Note that for fixed $s \in(0,1)$ we have that the map

$$
\begin{aligned}
& \mathcal{S} \longrightarrow \mathbb{C} \\
& z \longmapsto w_{0}^{\frac{z-\theta}{q}}(s) w_{1}^{\frac{\theta-z}{q}}(s)
\end{aligned}
$$

is holomorphic on $\mathcal{S}$. It follows from Cauchy's Integral Theorem (Theorem 1.4.2) that

$$
\int_{\gamma} w_{0}^{\frac{z-\theta}{q}}(s) w_{1}^{\frac{\theta-z}{q}}(s) d z=0
$$

Thus, $\int_{\gamma} x^{*}(\Phi(f)(z)) d z=0$. From Morera's Theorem (Theorem 1.4.3), we have that $x^{*}(\Phi(f)(z))$ is holomorphic on $\mathcal{S}$. Therefore, we have the desired result.

It follows from (3.10) that for each $f \in S_{L_{q}(w)}, \Phi(f)(j+i t) \in L_{q}\left(w_{j}\right), j=0,1, t \in \mathbb{R}$. Moreover, since $\Phi(f)$ is holomorphic over $\mathcal{S}$, it is continuous (Proposition 1.4.10). Thus, the mappings

$$
\begin{aligned}
\mathbb{R} & \longrightarrow L_{q}\left(w_{j}\right) \\
t \longmapsto & \longmapsto(f)(j+i t)
\end{aligned}
$$

are Bochner-measurable for $j=0,1$.

From Remark 1.5.9 we have that for each $z \in \mathbb{S}$

$$
\Phi(f)(z)=\int_{\mathbb{R}} \Phi(f)(i t) d \mu_{z, 0}(t)+\int_{\mathbb{R}} \Phi(f)(1+i t) d \mu_{z, 1}(t)
$$


From now on, we will consider $\Phi(f)$ as the restriction to the strip $\overline{\mathbb{S}}$, i.e. $\Phi(f): \mathbb{S} \longrightarrow L_{q}\left(w_{0}\right)+$ $L_{q}\left(w_{1}\right)$.

Note that for $t \in \mathbb{R}$, we have

$$
\begin{aligned}
\|\Phi(f)(i t)\|_{L_{q}\left(w_{0}\right)}^{q} & =\int|f|^{q}\left|w_{0}^{\frac{i t-\theta}{q}} w_{1}^{\frac{\theta-i t}{q}}\right|^{q} w_{0} d m \\
& =\int|f|^{q} w_{0}^{-\theta} w_{1}^{\theta} w_{0} d m=\int|f|^{q} w_{0}^{1-\theta} w_{1}^{\theta} d m=\|f\|_{L_{q}(w)}^{q}=1 \\
\|\Phi(f)(1+i t)\|_{L_{q}\left(w_{1}\right)}^{q} & =\int|f|^{q}\left|w_{0}^{\frac{1+i t-\theta}{q}} w_{1}^{\frac{\theta-1-i t}{q}}\right|^{q} w_{1} d m \\
& =\int|f|^{q} w_{0}^{1-\theta} w_{1}^{\theta-1} w_{1} d m=\int|f|^{q} w_{0}^{1-\theta} w_{1}^{\theta} d m=\|f\|_{L_{q}(w)}^{q}=1
\end{aligned}
$$

Consequently,

$$
\begin{aligned}
\|\Phi(f)\|_{\mathcal{F}_{\theta}^{p}(\bar{A})}^{p} & =\int_{\mathbb{R}}\|\Phi(f)(i t)\|_{L_{q}\left(w_{0}\right)}^{p} d \mu_{\theta, 0}(t)+\int_{\mathbb{R}}\|\Phi(f)(1+i t)\|_{L_{q}\left(w_{1}\right)}^{p} d \mu_{\theta, 1}(t) \\
& =\|f\|_{L_{q}(w)}^{p} \int_{\mathbb{R}} d \mu_{\theta, 0}(t)+\|f\|_{L_{q}(w)}^{p} \int_{\mathbb{R}} d \mu_{\theta, 1}(t)=(1-\theta)+\theta=1
\end{aligned}
$$

Thus, $\Phi(f) \in \mathcal{F}_{\theta}^{p}(\bar{A})$. Moreover, $\Phi(f)(\theta)=f$ and $\|\Phi(f)\|_{\mathcal{F}_{\theta}^{p}(\bar{A})}=1$. By the uniqueness of the minimal function in the Proposition 3.2.3 it follows that $\Gamma(f)=\Phi(f)$, for all $f \in S_{L_{q}(w)}$, i.e. $\Phi: S_{L_{q}(w)} \longrightarrow \mathcal{F}_{\theta}^{p}(\bar{A})$ is the minimal lifting map associated to $L_{q}(w)$.

Furthermore, according to Theorem 3.2.5 it follows that for $0<\theta_{1}, \theta_{2}<1$, the mapping

$$
\begin{aligned}
U: S_{L_{q}\left(w_{0}^{1-\theta_{1}} w_{1}^{\theta_{1}}\right)} & \longrightarrow S_{L_{q}\left(w_{0}^{1-\theta_{2}} w_{1}^{\theta_{2}}\right)} \\
f & \longmapsto f w_{0}^{\frac{\theta_{2}-\theta_{1}}{q}} w_{1}^{\frac{\theta_{1}-\theta_{2}}{q}}
\end{aligned}
$$

defines a uniform homemomorphism between the unit spheres of the interpolation spaces $L_{q}\left(w_{0}^{1-\theta_{1}} w_{1}^{\theta_{1}}\right)$ and $L_{q}\left(w_{0}^{1-\theta_{2}} w_{1}^{\theta_{2}}\right)$.

\subsection{Vertical and Horizontal Maps}

Looking at the minimal functions given by Proposition 3.2.3, we can consider vertical maps: instead of taking the minimal functions applied to a $\theta \in(0,1)$, we can consider, for fixed $t \in \mathbb{R}$, the minimal functions applied to $\theta+i t$; that is, the vertical maps are the functions

$$
\begin{aligned}
A_{\theta} & \longrightarrow A_{\theta} \\
\Gamma(f)(\theta)=f & \longmapsto \Gamma(f)(\theta+i t)
\end{aligned}
$$

Note that if we restrict the application above to the unit sphere of $A_{\theta}$ we have a uniform automorphism. 


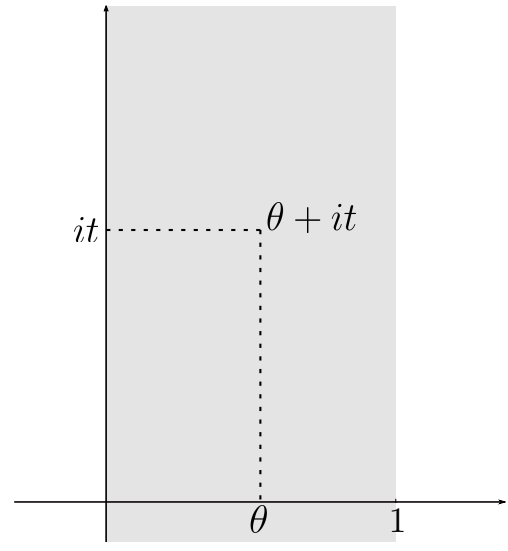

Example 3.5.1. Let $m$ denote the Lebesgue measure on $\Omega=(0,1), \mathcal{M}$ the Borel $\sigma$-algebra on $(0,1)$, and for $1 \leq p \leq \infty$ we put $L_{p}=L_{p}(\Omega, \mathcal{M}, m)$. For $0<\theta<1$ and $1<p_{0}<p_{1}<\infty$, we have from Theorem 2.4.1 that

$$
\left(L_{p_{0}}, L_{p_{1}}\right)_{\theta}=L_{p_{\theta}}, \quad \text { with } \frac{1}{p_{\theta}}=\frac{1-\theta}{p_{0}}+\frac{\theta}{p_{1}} .
$$

Moreover, we saw in Section 3.3 that the minimal lifting map $\Gamma: S_{L_{p_{\theta}}} \longrightarrow \mathcal{F}_{\theta}^{p}(\bar{A})$ is given by

$$
\Gamma(f)(z)=\operatorname{sgn}(f)|f|^{p_{\theta}\left(\frac{1-z}{p_{0}}+\frac{z}{p_{1}}\right)},
$$

for every $f \in S_{L_{p_{\theta}}}$ and $z \in \overline{\mathbb{S}}$. Thus, for $t \in \mathbb{R}$ the vertical map is given by

$$
\begin{aligned}
V_{t}: L_{p_{\theta}} & \longrightarrow L_{p_{\theta}} \\
f & \longmapsto f|f|^{p_{\theta}\left(\frac{-1}{p_{0}}+\frac{1}{p_{1}}\right) i t}
\end{aligned}
$$

Note that $V_{t}(f)$ equals $f$ times a unimodular function, for each $f \in L_{p_{\theta}}$.

We can also consider the horizontal maps that arise at different levels of the scale: that is, taking $\theta_{1}, \theta_{2} \in(0,1)$, and fixed $t \in \mathbb{R}$, consider the map

$$
\begin{aligned}
A_{\theta_{1}} & \longrightarrow A_{\theta_{2}} \\
\Gamma(f)\left(\theta_{1}+i t\right) & \longmapsto \Gamma(f)\left(\theta_{2}+i t\right)
\end{aligned}
$$




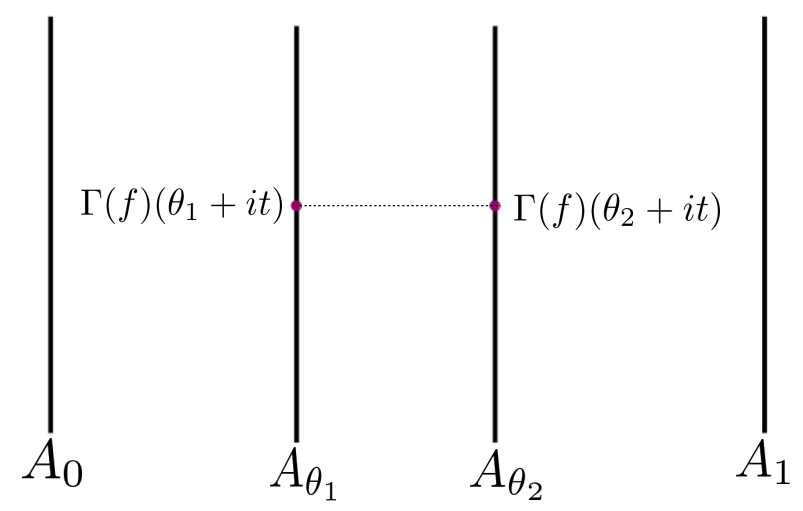

Let us analyze the horizontal maps on the interpolation scale of Lebesgue spaces. Let $m$ denote the Lebesgue measure in $\Omega=(0,1), \mathcal{M}$ the Borel $\sigma$-algebra on $(0,1)$, and for $1 \leq p \leq \infty$ we put $L_{p}=L_{p}(\Omega, \mathcal{M}, m)$.

Consider $A_{0}=L_{p_{0}}$ and $A_{1}=L_{p_{1}}$, with $1<p_{0}<p_{1}<+\infty$. Let $\theta_{1}, \theta_{2} \in(0,1)$, and define $1<p_{\theta_{1}}, p_{\theta_{2}}<\infty$ such that

$$
\frac{1}{p_{\theta_{j}}}=\frac{1-\theta_{j}}{p_{0}}+\frac{\theta_{j}}{p_{1}}, j=1,2
$$

Denote by $M$ the Mazur map

$$
\begin{aligned}
M: S_{L_{p_{\theta_{1}}}} & \longrightarrow S_{L_{p_{\theta_{2}}}} \\
f & \longmapsto \operatorname{sgn}(f)|f|^{p_{\theta_{1}} / p_{\theta_{2}}}
\end{aligned}
$$

The minimal lifting map $\Gamma: S_{L_{p_{1}}} \longrightarrow \mathcal{F}_{\theta_{1}}^{p}(\bar{A})$ associated to the space $L_{p_{\theta_{1}}}$ is given by $\Gamma(f)(z)=$ $\operatorname{sgn}(f)|f|^{p_{\theta_{1}}\left(\frac{1-z}{p_{0}}+\frac{z}{p_{1}}\right)}$, for every $f \in S_{L_{p_{\theta_{1}}}}$ and $z \in \overline{\mathbb{S}}$.

Proposition 3.5.2. For every $t \in \mathbb{R}$, the horizontal map

$$
\begin{aligned}
H_{t}: S_{L_{p_{\theta_{1}}}} & \longrightarrow S_{L_{p_{\theta_{2}}}} \\
f=\Gamma(g)\left(\theta_{1}+i t\right) & \longmapsto \Gamma(g)\left(\theta_{2}+i t\right)
\end{aligned}
$$

coincides with the Mazur map and therefore is a uniform homeomorphism between the spheres of interpolation spaces.

Proof. Let us first show that

$$
\begin{aligned}
& S_{L_{p_{\theta_{1}}}}=\left\{\Gamma(g)\left(\theta_{1}+i t\right): g \in S_{L_{p_{\theta_{1}}}}\right\}, \forall t \in \mathbb{R} \\
& S_{L_{p_{\theta_{2}}}}=\left\{\Gamma(g)\left(\theta_{2}+i t\right): g \in S_{L_{p_{\theta_{1}}}}\right\}, \forall t \in \mathbb{R}
\end{aligned}
$$


Indeed, if $g \in S_{L_{p_{\theta_{1}}}}$, we have

$$
\begin{aligned}
\Gamma(g)\left(\theta_{1}+i t\right) & =\operatorname{sgn}(g)|g|^{p_{\theta_{1}}\left(\frac{1-\left(\theta_{1}+i t\right)}{p_{0}}+\frac{\theta_{1}+i t}{p_{1}}\right)}=\operatorname{sgn}(g)|g|^{p_{\theta_{1}}\left(\frac{1-\theta_{1}}{p_{0}}+\frac{\theta_{1}}{p_{1}}\right)}|g|^{p_{\theta_{1}}\left(\frac{-i t}{p_{0}}+\frac{i t}{p_{1}}\right)} \\
& =\operatorname{sgn}(g)|g||g|^{p_{\theta_{1}}\left(\frac{-i t}{p_{0}}+\frac{i t}{p_{1}}\right)}=g|g|^{p_{\theta_{1}}\left(\frac{-i t}{p_{0}}+\frac{i t}{p_{1}}\right)}
\end{aligned}
$$

Then,

$$
\int\left|\Gamma(g)\left(\theta_{1}+i t\right)\right|^{p_{\theta_{1}}}=\int|g|^{p_{\theta_{1}}}=\|g\|_{L_{p_{\theta_{1}}}}^{p_{\theta_{1}}}=1
$$

Therefore, $\left\{\Gamma(g)\left(\theta_{1}\right): g \in S_{L_{p_{\theta_{1}}}}\right\} \subseteq S_{L_{p_{\theta_{1}}}}$. Now, let $f \in S_{L_{p_{\theta_{1}}}}$ and consider, $\forall s \in(0,1)$

$$
g(s)= \begin{cases}\frac{f(s)}{|f(s)|^{p_{1}}\left(\frac{-i t}{p_{0}}+\frac{i t}{p_{1}}\right)}, & \text { if } f(s) \neq 0 \\ 0, & \text { if } f(s)=0\end{cases}
$$

Note that

$$
\int|g|^{p_{\theta_{1}}}=\int\left|\frac{f(s)}{|f(s)|^{p_{\theta_{1}}\left(\frac{-i t}{p_{0}}+\frac{i t}{p_{1}}\right)}}\right|^{p_{\theta_{1}}}=\int|f|^{p_{\theta_{1}}}=\|f\|_{L_{p_{\theta_{1}}}^{p_{\theta_{1}}}}^{p_{1}}=1
$$

thus, $g \in S_{L_{p_{1}}}$ and

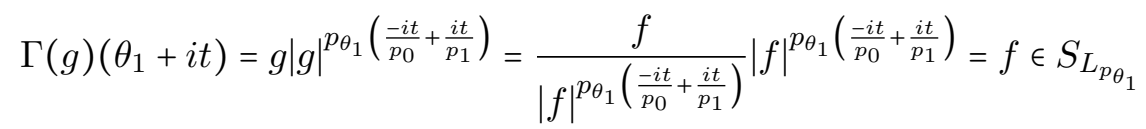

and this proves (3.11). Similarly, we can prove (3.12). Thus, $H_{t}: S_{L_{p_{\theta_{1}}}} \longrightarrow S_{L_{p_{\theta_{2}}}}$.

Note that $H_{t}$ is well defined. Indeed, suppose that $g, h \in S_{L_{p_{\theta_{1}}}}$ are such that

$$
\Gamma(g)\left(\theta_{1}+i t\right)=\Gamma(h)\left(\theta_{1}+i t\right) .
$$

Let us show that $\Gamma(g)\left(\theta_{2}+i t\right)=\Gamma(h)\left(\theta_{2}+i t\right)$. We have that

$$
\Gamma(g)\left(\theta_{1}+i t\right)=g|g|^{p_{\theta_{1}}\left(\frac{-i t}{p_{0}}+\frac{i t}{p_{1}}\right)}=h|h|^{p_{\theta_{1}}\left(\frac{-i t}{p_{0}}+\frac{i t}{p_{1}}\right)}=\Gamma(h)\left(\theta_{1}+i t\right),
$$

which implies $\left|\Gamma(g)\left(\theta_{1}+i t\right)\right|=|g|=|h|=\left|\Gamma(h)\left(\theta_{1}+i t\right)\right|$ and

$$
\operatorname{sgn}\left(\Gamma(g)\left(\theta_{1}+i t\right)\right)=\operatorname{sgn}(g)|g|^{p_{\theta_{1}}\left(\frac{-i t}{p_{0}}+\frac{i t}{p_{1}}\right)}=\operatorname{sgn}(h)|h|^{p_{\theta_{1}}\left(\frac{-i t}{p_{0}}+\frac{i t}{p_{1}}\right)}=\operatorname{sgn}\left(\Gamma(h)\left(\theta_{1}+i t\right)\right) .
$$

Thus,

$$
\begin{aligned}
H_{t}\left(\Gamma(g)\left(\theta_{1}+i t\right)\right) & =\Gamma(h)\left(\theta_{2}+i t\right)=\operatorname{sgn}(h)|h|^{p_{\theta_{1}} / p_{\theta_{2}}}|h|^{p_{\theta_{1}}\left(\frac{-i t}{p_{0}}+\frac{i t}{p_{1}}\right)} \\
& =\Gamma(g)\left(\theta_{2}+i t\right)=H_{t}\left(\Gamma(h)\left(\theta_{1}+i t\right)\right)
\end{aligned}
$$

Finally, let us show that that $H_{t}(f)=M(f)$ for all $f \in S_{L_{p_{1}}}$. Note that, if $f=\Gamma(g)\left(\theta_{1}+i t\right)$, then

$$
\operatorname{sgn}(f)=\operatorname{sgn}\left(\Gamma(g)\left(\theta_{1}+i t\right)\right)=\operatorname{sgn}(g)|g|^{p_{\theta_{1}}\left(\frac{-i t}{p_{0}}+\frac{i t}{p_{1}}\right)}
$$


and

$$
|f|=\left|\Gamma(g)\left(\theta_{1}+i t\right)\right|=|g|
$$

So we have

$$
\begin{aligned}
M(f) & =M\left(\Gamma(g)\left(\theta_{1}+i t\right)\right)=\operatorname{sgn}(f)|f|^{p_{\theta_{1}} / p_{\theta_{2}}} \\
& =\operatorname{sgn}(g)|g|^{p_{\theta_{1}}\left(\frac{-i t}{p_{0}}+\frac{i t}{p_{1}}\right)}|g|^{p_{\theta_{1}} / p_{\theta_{2}}}=\Gamma(g)\left(\theta_{2}+i t\right)=H_{t}(f)
\end{aligned}
$$

Therefore, $H_{t}=M$.

\subsection{Notes and Comments}

If $X_{1}$ and $X_{2}$ are two isomorphic Banach spaces, then $S_{X_{1}}$ and $S_{X_{2}}$ are uniformly homeomorphic. Indeed, if $\Psi: X_{1} \longrightarrow X_{2}$ defines an isomorphism, notice that

$$
\begin{aligned}
U: S_{X_{1}} & \longrightarrow S_{X_{2}} \\
x & \longmapsto \frac{\Psi(x)}{\|\Psi(x)\|_{X_{2}}}
\end{aligned}
$$

defines a uniformly continuous function, whose inverse is given by

$$
\begin{aligned}
U^{-1}: S_{X_{2}} & \longrightarrow S_{X_{1}} \\
y & \longmapsto \frac{\Psi^{-1}(y)}{\left\|\Psi^{-1}(y)\right\|_{X_{1}}} .
\end{aligned}
$$

As we saw in Section 2.6, the interpolation of two Sobolev (respectively, Orlicz) spaces gives us a Sobolev (respectively, Orlicz) space with an equivalent norm (that is, gives us an interpolation space that is isomorphic to a Sobolev or Orlicz space). Thus, according to what we have seen above, Daher's Theorem still applies, that is, the unit spheres between two interpolation spaces on the Sobolev or Orlicz scale are uniformly homeomorphic. However, the lack of an isometry makes it more difficult to explicit the form of the minimal functions. 



\section{Chapter 4}

\section{Banach Lattices}

Many of the naturally occurring Banach spaces carry additional structures other than the linear and metric structure. This additional structure may affect properties that are stated completely in terms of Banach space concepts (such as linear operators and duality). An additional structure that can be considered is the lattice structure. The concept of lattice involves ordering the space partially. Banach lattices provide a way to study classical vector spaces such as $L_{p}$ spaces and Lorentz spaces. In this chapter, we will introduce the concept and constructions of Banach lattices based on the article [Cal64].

In this chapter, we consider $(\Omega, \mathcal{M}, \mu)$ a $\sigma$-finite measure space. We denote by $L_{0} \doteq L_{0}(\Omega, \mathcal{M}, \mu)$ the class of all measurable functions $f: \Omega \longrightarrow \mathbb{C}$. In $L_{0}$ we identify the functions that are equal $\mu$-a.e. For $1 \leq p \leq \infty$, put $L_{p} \doteq L_{p}(\Omega, \mathcal{M}, \mu)$.

\subsection{Initial Definitions}

Definition 4.1.1. A subclass $X$ of $L_{0}$ is a Banach lattice on $L_{0}$ if

(i) $X$ is a Banach space.

(ii) If $f \in X$ and $|g| \leq|f| \mu$-a.e. we have that $g \in X$ and $\|g\|_{X} \leq\|f\|_{X}$.

An immediate example is the case of $L_{p}$ spaces.

Example 4.1.2. For $1 \leq p \leq \infty, L_{p}$ is a Banach lattice.

Indeed, if $f \in L_{p}$ and $|g| \leq|f| \mu$-a.e., then for $p<\infty$ we have

$$
\int|g|^{p} d \mu \leq \int|f|^{p} d \mu
$$

which implies that $g \in L_{p}$ and $\|g\|_{L_{p}} \leq\|f\|_{L_{p}}$. If $p=\infty$, consider $\lambda \geq 0$ such $|f| \leq \lambda \mu$-a.e., then we have $|g| \leq|f| \leq \lambda \mu$-a.e., that is, $g$ is essentially bounded and $\|g\|_{L_{\infty}} \leq\|f\|_{L_{\infty}}$.

We want to consider different constructions of Banach lattices (such as the Calderón-Lozanovskii 
construction). The next lemmas will be useful to prove the completeness of the spaces that we will define.

Lemma 4.1.3. Let $X$ be a Banach lattice and consider a sequence $\left(f_{n}\right)$ in $X$ such that $\sum_{n}\left\|f_{n}\right\|_{X}<$ $\infty$. Then there is $f \in X$ such that the series $\sum_{n} f_{n}(x)$ is absolutely convergent and $\sum_{n} f_{n}(x)=f(x)$ $\mu$-a.e.

Proof. See Section 33.2 in [Cal64].

Definition 4.1.4. Consider $I \subseteq \mathbb{R}$ an interval. A function $\varphi: I \longrightarrow \mathbb{R}$ is said to be concave if for any $x, y \in I$ and $\lambda \in[0,1]$ we have

$$
(1-\lambda) \varphi(x)+\lambda \varphi(y) \leq \varphi((1-\lambda) x+\lambda y)
$$

Lemma 4.1.5. (Jensen's inequality for concave functions) Let $\varphi:[0, \infty) \longrightarrow \mathbb{R}$ be a concave non-negative function such that $\varphi(0)=0$. Let $\left(\lambda_{n}\right)$ and $\left(a_{n}\right)$ be two sequences of positive numbers and suppose that $\sum_{n} \lambda_{n}<\infty$. Then we have

$$
\frac{\sum_{n} \lambda_{n} \varphi\left(a_{n}\right)}{\sum_{n} \lambda_{n}} \leq \varphi\left(\frac{\sum_{n} \lambda_{n} a_{n}}{\sum_{n} \lambda_{n}}\right)
$$

\subsection{Construction of Banach lattices}

Consider $X$ a Banach lattice on $L_{0}$ and $\varphi: \Omega \times[0, \infty) \longrightarrow \mathbb{R}$ such that for each $x \in \Omega, \varphi(x, \cdot)$ is an increasing concave function and $\varphi(x, 0)=0$. Consider

$$
\varphi(X)=\left\{g \in L_{0}: \exists \lambda>0 \text { and } f \in B_{X}, f \geq 0 \text { such that }|g(x)| \leq \lambda \varphi(x, f(x)) \mu \text {-a.e. }\right\}
$$

equipped with the norm

$$
\|g\|_{\varphi(X)}=\inf \left\{\lambda>0:|g(x)| \leq \lambda \varphi(x, f(x)) \mu \text {-a.e. for some } f \in B_{X}, f \geq 0\right\} .
$$

To better understand what we are dealing with, let us first consider an example. We will see in Section 4.3 that the construction above, considering a specific $\varphi$, coincides with what we call $p$ convexification.

Example 4.2.1. If $X=L_{1}$ and $\varphi(x, t)=t^{\frac{1}{p}}$, for $1<p<\infty$, then $\varphi(X)=L_{p}$.

Let $g \in \varphi(X)$, then there are $f \in B_{X}, f \geq 0, \lambda>0$ such that $|g| \leq \lambda|f|^{\frac{1}{p}}, \mu$-a.e. Therefore,

$$
\int|g|^{p} d \mu \leq \lambda^{p} \int|f| d \mu=\lambda^{p}\|f\|_{L_{1}}<\infty
$$

Thus, $g \in L_{p}$. Equation (4.1) also implies that $\|g\|_{L_{p}} \leq\|g\|_{\varphi(X)}$. 
On the other hand, if $g \in L_{p}$, with $g \neq 0$, consider

$$
f=\frac{|g|^{p}}{\|g\|_{L_{p}}^{p}} \text { and } \lambda=\|g\|_{L_{p}}>0
$$

then $f \in B_{X}$. Moreover

$$
\lambda|f|^{\frac{1}{p}}=\|g\|_{L_{p}}\left(\frac{|g|^{p}}{\int|g|^{p} d \mu}\right)^{\frac{1}{p}}=|g|
$$

Thus, $g \in \varphi(X)$. Equation (4.2) also implies that $\|g\|_{\varphi(X)} \leq\|g\|_{L_{p}}$.

Hence, $\varphi(X)=L_{p}$, with equality of norms.

Theorem 4.2.2. $\left(\varphi(X),\|\cdot\|_{\varphi(X)}\right)$ is a Banach lattice.

Proof. Let us first show that $\|\cdot\|_{\varphi(X)}$ defines a norm on $\varphi(X)$.

(i) Let $g \in \varphi(X)$ and $\alpha \in \mathbb{K}$. Consider

$$
\begin{gathered}
S_{1}=\left\{\lambda:|\alpha g(x)| \leq \lambda \varphi(x, f(x)) \mu \text {-a.e. for some } f \in B_{X}, f \geq 0\right\} \\
S_{2}=\left\{|\alpha| \lambda:|g(x)| \leq \lambda \varphi(x, f(x)) \mu \text {-a.e. for some } f \in B_{X}, f \geq 0\right\}
\end{gathered}
$$

Then we have that $\|\alpha g\|_{\varphi(X)}=\inf S_{1}$ and $|\alpha|\|g\|_{\varphi(X)}=\inf S_{2}$. If $\lambda \in S_{1}$, then there is $f \in B_{X}$, $f \geq 0$, such that $|\alpha g(x)| \leq \lambda \varphi(x, f(x)) \mu$-a.e. Taking $\beta=\frac{\lambda}{|\alpha|}$, we have that $|\alpha| \beta$ satisfies $|g(x)| \leq \beta \varphi(x, f(x)) \mu$-a.e., therefore, $\lambda=|\alpha| \beta \in S_{2}$, i.e., $S_{1} \subseteq S_{2}$. Hence $|\alpha|\|g\|_{\varphi(X)}=\inf S_{2} \leq$ $\inf S_{1}=\|\alpha g\|_{\varphi(X)}$. Similarly, we can prove that $S_{1} \subseteq S_{2}$ and we have $|\alpha|\|g\|_{\varphi(X)}=\|\alpha g\|_{\varphi(X)}$.

(ii) Consider $g_{1}, g_{2} \in \varphi(X)$. For every $\epsilon>0$ there are $\lambda_{1}, \lambda_{2}>0, f_{1}, f_{2} \in B_{X}, f_{1}, f_{2} \geq 0$ such that

$$
\lambda_{j} \leq\left\|g_{j}\right\|_{\varphi(X)}+\frac{\epsilon}{2} \quad \text { and } \quad\left|g_{j}\right| \leq \lambda_{j} \varphi\left(x, f_{j}\right), \mu \text {-a.e., } j=0,1
$$

then, from Lemma 4.1.5 we have that

$$
\left|g_{1}\right|+\left|g_{2}\right| \leq \lambda_{1} \varphi\left(x, f_{1}(x)\right)+\lambda_{2} \varphi\left(x, f_{2}(x)\right) \leq\left(\lambda_{1}+\lambda_{2}\right)\left[\varphi\left(x, \frac{\lambda_{1} f_{1}+\lambda_{2} f_{2}}{\lambda_{1}+\lambda_{2}}\right)\right] \mu \text {-a.e. }
$$

Note that, since $\frac{\lambda_{1} f_{1}+\lambda_{2} f_{2}}{\lambda_{1}+\lambda_{2}} \in B_{X}$, we have

$$
\left\|\left|g_{1}\right|+\left|g_{2}\right|\right\|_{\varphi(X)} \leq \lambda_{1}+\lambda_{2} \leq\left\|g_{1}\right\|_{\varphi(X)}+\left\|g_{2}\right\|_{\varphi(X)}+\epsilon
$$

As $\epsilon>0$ is arbitrary

$$
\left\|\left|g_{1}\right|+\left|g_{2}\right|\right\|_{\varphi(X)} \leq\left\|g_{1}\right\|_{\varphi(X)}+\left\|g_{2}\right\|_{\varphi(X)}
$$

Now, from the definitions of $\left\|\left|g_{1}\right|+\left|g_{2}\right|\right\|_{\varphi(X)}$ and $\left\|g_{1}+g_{2}\right\|_{\varphi(X)}$ and the fact that $\left|g_{1}+g_{2}\right| \leq$ $\left|g_{1}\right|+\left|g_{2}\right|$ we have

$$
\left\|g_{1}+g_{2}\right\|_{\varphi(X)} \leq\left\|\left|g_{1}\right|+\left|g_{2}\right|\right\|_{\varphi(X)} \leq\left\|g_{1}\right\|_{\varphi(X)}+\left\|g_{2}\right\|_{\varphi(X)} .
$$


(iii) Finally, consider $g \in \varphi(X)$ such that $\|g\|_{\varphi(X)}=0$. Let us show that $g=0 \mu$-a.e. We have that

$$
0=\|g\|_{\varphi(X)}=\inf \left\{\lambda:|g(x)| \leq \lambda \varphi(x, f(x)) \mu \text {-a.e. for some } f \in B_{X}, f \geq 0\right\} .
$$

Therefore, by the infimum property, for each $n \in \mathbb{N}$, there are $f_{n} \in B_{X}, f_{n} \geq 0$, such that

$$
|g(x)| \leq \frac{1}{n^{3}} \varphi\left(x, f_{n}(x)\right), \mu \text {-a.e. }
$$

From the concavity of $\varphi(x, t)$ in $t$ and $\varphi(x, 0)=0$ we have, for all $0 \leq \lambda \leq 1$, that

$$
\varphi(x, \lambda t)=\varphi(x, \lambda t+(1-\lambda) 0) \geq \lambda \varphi(x, t)+(1-\lambda) \varphi(x, 0)=\lambda \varphi(x, t),
$$

that is, $\varphi(x, \lambda t) \geq \lambda \varphi(x, t)$. Therefore, for each $n \in \mathbb{N}$

$$
|g| \leq \frac{1}{n^{3}} \varphi\left(x, f_{n}\right) \leq \frac{1}{n} \varphi\left(x, \frac{1}{n^{2}} f_{n}\right), \mu \text {-a.e. }
$$

i.e., for each $n \in \mathbb{N}$ there are sets $M_{n} \subseteq \Omega$, with $\mu\left(M_{n}^{c}\right)=0$ such that $|g| \leq \frac{1}{n^{3}} \varphi\left(x, f_{n}\right) \leq$ $\frac{1}{n} \varphi\left(x, \frac{1}{n^{2}} f_{n}\right)$, for all $x \in M_{n}$. Taking $M=\bigcap_{n} M_{n}$, it follows that $\mu\left(M^{c}\right)=0$ and

$$
|g| \leq \frac{1}{n^{3}} \varphi\left(x, f_{n}\right) \leq \frac{1}{n} \varphi\left(x, \frac{1}{n^{2}} f_{n}\right), \forall n \in \mathbb{N}, \forall x \in M .
$$

Moreover, since $\left\|\sum_{n} \frac{1}{n^{2}} f_{n}\right\|_{X} \leq \sum_{n} \frac{1}{n^{2}}\left\|f_{n}\right\|_{X} \leq \sum_{n} \frac{1}{n^{2}}<\infty$, it follows from Lemma 4.1.3 that $\sum_{n} \frac{1}{n^{2}} f_{n}(x)$ is convergent $\mu$-a.e., and in particular, $\frac{1}{n^{2}} f_{n}(x)$ converges to zero $\mu$-a.e., that is, there is a measurable set $N$ with $\mu\left(N^{c}\right)=0$ and such that

$$
\frac{1}{n^{2}} f_{n}(x)=0, \forall x \in N .
$$

Now, for $x \in M \cap N$, we have that $\frac{1}{n^{2}} f_{n}(x) \rightarrow 0$ when $n \rightarrow \infty$, and since $\varphi(x, t)$ is concave in $t$, it is continuous in $t$, then $\varphi\left(x, \frac{1}{n^{2}} f_{n}(x)\right) \rightarrow \varphi(x, 0)=0$. Thus, for all $x \in M \cap N$

$$
|g(x)| \leq \frac{1}{n} \varphi\left(x, \frac{1}{n^{2}} f_{n}(x)\right) \rightarrow 0 .
$$

Since $\mu\left((M \cap N)^{c}\right)=0$, it follows that $g=0 \mu$-a.e.

Now, let us show that the norm defined on $\varphi(X)$ is complete. Let $\left(g_{n}\right)$ be a sequence of functions in $\varphi(X)$ such that $\sum_{n}\left\|g_{n}\right\|_{\varphi(X)}<\infty$. From the infimum property, for every $\epsilon>0$ and for each $n \in \mathbb{N}$ there are $\lambda_{n}>0$ and functions $f_{n} \in B_{X}, f_{n} \geq 0$ such that

$$
\begin{gathered}
\lambda_{n} \leq\left\|g_{n}\right\|_{\varphi(X)}+\frac{\epsilon}{2^{n}} \\
\left|g_{n}(x)\right| \leq \lambda_{n} \varphi\left(x, f_{n}(x)\right), \mu \text {-a.e. }
\end{gathered}
$$

From Lemma 4.1.5, we have

$$
\sum_{n}\left|g_{n}(x)\right| \leq \sum_{n} \lambda_{n} \varphi\left(x, f_{n}(x)\right) \leq\left(\sum_{n} \lambda_{n}\right) \varphi\left(x, \frac{\sum_{n} \lambda_{n} f_{n}(x)}{\sum_{n} \lambda_{n}}\right), \mu \text {-a.e. }
$$


Note that

$$
\sum_{n}\left\|\lambda_{n} f_{n}\right\|_{X} \leq \sum_{n} \lambda_{n} \leq \sum_{n}\left\|g_{n}\right\|_{\varphi(X)}+\epsilon
$$

thus, from Lemma 4.1.3, there is $f \in X$ such that $\sum_{n} \lambda_{n} f_{n}(x)=f(x)$, $\mu$-a.e. Moreover, since $\frac{\sum_{n} \lambda_{n} f_{n}(x)}{\sum_{n} \lambda_{n}} \in B_{X}$, it follows from (4.3) that $\sum_{n}\left|g_{n}\right| \in \varphi(X)$. And since $\left|\sum_{n} g_{n}(x)\right| \leq \sum_{n}\left|g_{n}(x)\right|$, we have from the definition of $\varphi(X)$ that $\sum_{n} g_{n} \in \varphi(X)$.

Lastly, let us show that given a function $f \in \varphi(X)$ and $g$ such that $|g| \leq|f|$ then $g \in \varphi(X)$ and $\|g\|_{\varphi(X)} \leq\|f\|_{\varphi(X)}$. Indeed, since $f \in \varphi(X)$, there are $\lambda>0$ and $h \in B_{X}, h \geq 0$, such that $|f| \leq \lambda \varphi(x, h)$; thus

$$
|g| \leq|f| \leq \lambda \varphi(x, h)
$$

and $g \in \varphi(X)$. The inequality (4.4) also implies that

$$
\begin{aligned}
\|g\|_{\varphi(X)} & =\inf \left\{\lambda>0:|g(x)| \leq \lambda \varphi(x, h(x)) \mu \text {-a.e. for some } h \in B_{X}, h \geq 0\right\} \\
& \leq \inf \left\{\lambda>0:|f(x)| \leq \lambda \varphi(x, h(x)) \mu \text {-a.e. for some } h \in B_{X}, h \geq 0\right\}=\|f\|_{\varphi(X)} .
\end{aligned}
$$

\subsubsection{Calderón-Lozanovskii Construction}

Let us now introduce the Calderón-Lozanovskii Construction for Banach lattices. Later we will see that such a construction, under certain conditions, coincides with the complex interpolation space.

Consider $X_{1}, X_{2}$ two Banach lattices on $L_{0}$ and $0<\theta<1$. Define

$$
X=X_{1}^{1-\theta} X_{2}^{\theta}=\left\{f \in L_{0}:|f(x)| \leq \lambda|g(x)|^{1-\theta}|h(x)|^{\theta} \mu \text {-a.e., for some } \lambda>0 \text { and } g \in B_{X_{1}}, h \in B_{X_{2}}\right\}
$$

For each $f \in X$, we define

$$
\|f\|_{X}=\inf \left\{\lambda:|f(x)| \leq \lambda|g(x)|^{1-\theta}|h(x)|^{\theta} \mu \text {-a.e., such that } g \in B_{X_{1}}, h \in B_{X_{2}}\right\} .
$$

Theorem 4.2.3. $\left(X,\|\cdot\|_{X}\right)$ is a Banach lattice on $L_{0}$.

Proof. Let us first show that $\|\cdot\|_{X}$ defines a norm in $X$.

(i) Given $f \in X$ and $\alpha \in \mathbb{R}$, the proof that $\|\alpha f\|_{X}=|\alpha|\|f\|_{X}$ is similar to (i) in the proof of Theorem 4.2.2.

(ii) Let $f_{1}, f_{2} \in X$. Then, for each $\epsilon>0$ there are $\lambda_{1}, \lambda_{2}>0, g_{1}, g_{2} \in B_{X_{1}}$ and $h_{1}, h_{2} \in B_{X_{2}}$ such that

$$
\lambda_{i} \leq\|f\|_{X_{i}}+\frac{\epsilon}{2}, \quad \text { and }\left|f_{i}\right| \leq \lambda_{i}\left|g_{i}\right|^{1-\theta}\left|h_{i}\right|^{\theta}, \mu \text {-a.e., } i=1,2
$$


Taking $p=\frac{1}{1-\theta}$ and $q=\frac{1}{\theta}$, we have from the Hölder's Inequality (Theorem 1.2.2) that

$$
\begin{aligned}
\left|f_{1}\right|+\left|f_{2}\right| & \leq \lambda_{1}\left|g_{1}\right|^{1-\theta}\left|h_{1}\right|^{\theta}+\lambda_{2}\left|g_{2}\right|^{1-\theta}\left|h_{2}\right|^{\theta}=\lambda_{1}^{1-\theta} \lambda_{1}^{\theta}\left|g_{1}\right|^{1-\theta}\left|h_{1}\right|^{\theta}+\lambda_{2}^{1-\theta} \lambda_{2}^{\theta}\left|g_{2}\right|^{1-\theta}\left|h_{2}\right|^{\theta} \\
& \leq\left[\left(\lambda_{1}^{1-\theta}\left|g_{1}\right|^{1-\theta}\right)^{\frac{1}{1-\theta}}+\left(\lambda_{2}^{1-\theta}\left|g_{2}\right|^{1-\theta}\right)^{\frac{1}{1-\theta}}\right]^{1-\theta}\left[\left(\lambda_{1}^{\theta}\left|h_{1}\right|^{\theta}\right)^{\frac{1}{\theta}}+\left(\lambda_{2}^{\theta}\left|h_{2}\right|^{\theta}\right)^{\frac{1}{\theta}}\right]^{\theta} \\
& =\left(\lambda_{1}\left|g_{1}\right|+\lambda_{2}\left|g_{2}\right|\right)^{1-\theta}\left(\lambda_{1}\left|h_{1}\right|+\lambda_{2}\left|h_{2}\right|\right)^{\theta} \\
& =\left(\lambda_{1}+\lambda_{2}\right)^{1-\theta}\left(\frac{\lambda_{1}\left|g_{1}\right|+\lambda_{2}\left|g_{2}\right|}{\lambda_{1}+\lambda_{2}}\right)^{1-\theta}\left(\lambda_{1}+\lambda_{2}\right)^{\theta}\left(\frac{\lambda_{1}\left|h_{1}\right|+\lambda_{2}\left|h_{2}\right|}{\lambda_{1}+\lambda_{2}}\right)^{\theta} \\
& =\left(\lambda_{1}+\lambda_{2}\right)\left(\frac{\lambda_{1}\left|g_{1}\right|+\lambda_{2}\left|g_{2}\right|}{\lambda_{1}+\lambda_{2}}\right)^{1-\theta}\left(\frac{\lambda_{1}\left|h_{1}\right|+\lambda_{2}\left|h_{2}\right|}{\lambda_{1}+\lambda_{2}}\right)^{\theta}
\end{aligned}
$$

Therefore

$$
\left|f_{1}\right|+\left|f_{2}\right| \leq\left(\lambda_{1}+\lambda_{2}\right)\left(\frac{\lambda_{1}\left|g_{1}\right|+\lambda_{2}\left|g_{2}\right|}{\lambda_{1}+\lambda_{2}}\right)^{1-\theta}\left(\frac{\lambda_{1}\left|h_{1}\right|+\lambda_{2}\left|h_{2}\right|}{\lambda_{1}+\lambda_{2}}\right)^{\theta}
$$

Note that, since $\frac{\lambda_{1}\left|g_{1}\right|+\lambda_{2}\left|g_{2}\right|}{\lambda_{1}+\lambda_{2}} \in B_{X_{1}}$ and $\frac{\lambda_{1}\left|h_{1}\right|+\lambda_{2}\left|h_{2}\right|}{\lambda_{1}+\lambda_{2}} \in B_{X_{2}}$, it follows from the definition of $X$ and equation (4.5) that $\left|f_{1}\right|+\left|f_{2}\right| \in X$ and $\left\|\left|f_{1}\right|+\left|f_{2}\right|\right\|_{X} \leq \lambda_{1}+\lambda_{2}$. Then

$$
\left\|f_{1}+f_{2}\right\|_{X} \leq\left\|\left|f_{1}\right|+\left|f_{2}\right|\right\|_{X} \leq \lambda_{1}+\lambda_{2} \leq\left\|f_{1}\right\|_{X}+\left\|f_{2}\right\|_{X}+\epsilon \text {. }
$$

Since $\epsilon>0$ is arbitrary, we have $\left\|f_{1}+f_{2}\right\|_{X} \leq\left\|f_{1}\right\|_{X}+\left\|f_{2}\right\|_{X}$.

(iii) Finally, let $f \in X$ be such that $\|\cdot\|_{X}=0$. Let us show that $f=0 \mu$-a.e. By the definition of $\|\cdot\|_{X}$, for each $n \in \mathbb{N}$ there are $\lambda_{n} \leq \frac{1}{n^{2}}, g_{n} \in B_{X_{1}}$ and $h_{n} \in B_{X_{2}}$ such that

$$
|f| \leq \lambda_{n}\left|g_{n}\right|^{1-\theta}\left|h_{n}\right|^{\theta}, \quad \mu \text {-a.e. }
$$

Therefore

$$
|f| \leq \lambda_{n}\left|g_{n}\right|^{1-\theta}\left|h_{n}\right|^{\theta} \leq \frac{1}{n^{2}}\left|g_{n}\right|^{1-\theta}\left|h_{n}\right|^{\theta}=\left|\frac{1}{n^{2}} g_{n}\right|^{1-\theta}\left|\frac{1}{n^{2}} h_{n}\right|^{\theta}, \mu \text {-a.e. }
$$

Note that $\sum_{n}\left\|\frac{1}{n^{2}} g_{n}\right\|_{X_{1}}<\infty$ and $\sum_{n}\left\|\frac{1}{n^{2}} h_{n}\right\|_{X_{1}}<\infty$. Therefore, from Lemma 4.1.3 it follows that $\frac{1}{n^{2}} g_{n} \rightarrow 0$ and $\frac{1}{n^{2}} h_{n} \rightarrow 0 \mu$-a.e. and combining this with the inequality (4.6), we have that $f=0 \mu$-a.e.

In order to show that $X$ is a Banach space, consider a sequence $\left(f_{n}\right)$ in $X$ such that $\sum_{n}\left\|f_{n}\right\|_{X}<\infty$. From the definition of the norm, for each $\epsilon>0$ there are sequences $\left(\lambda_{n}\right)$ in $\mathbb{R}^{+},\left(g_{n}\right)$ in $B_{X_{1}}$ and $\left(h_{n}\right)$ in $B_{X_{2}}$ such that

$$
\lambda_{n} \leq\left\|f_{n}\right\|_{X}+\frac{\epsilon}{2^{n}} \text { and }\left|f_{n}\right| \leq \lambda_{n}\left|g_{n}\right|^{1-\theta}\left|h_{n}\right|^{\theta}, \mu \text {-a.e. }
$$


From Hölder's Inequality (Theorem 1.2.2) we have that

$$
\begin{aligned}
\sum_{n}\left|f_{n}\right| & \leq\left(\sum_{n} \lambda_{n}\left|g_{n}\right|\right)^{1-\theta}\left(\sum_{n} \lambda_{n}\left|h_{n}\right|\right)^{\theta} \\
& \leq\left(\sum_{n} \lambda_{n}\right)\left[\left(\sum_{n} \lambda_{n}\left|g_{n}\right|\right)\left(\sum_{n} \lambda_{n}\right)^{-1}\right]^{1-\theta}\left[\left(\sum_{n} \lambda_{n}\left|h_{n}\right|\right)\left(\sum_{n} \lambda_{n}\right)^{-1}\right]^{\theta}, \mu \text {-a.e. }
\end{aligned}
$$

Therefore, $\sum_{n}\left|f_{n}\right|<\infty \mu$-a.e..

Moreover, since $\left(\sum_{n} \lambda_{n}\left|g_{n}\right|\right)\left(\sum_{n} \lambda_{n}\right)^{-1} \in B_{X_{1}}$ and $\left(\sum_{n} \lambda_{n}\left|h_{n}\right|\right)\left(\sum_{n} \lambda_{n}\right)^{-1} \in B_{X_{2}}$, from the definition of $X$, it follows that $\sum_{n}\left|f_{n}\right| \in X$. Let $f(x)=\sum_{n} f_{n}(x)$ (where we define $f$ to be zero at the points where the series is not convergent). Since $|f| \leq \sum_{n}\left|f_{n}\right| \mu$-a.e., $f \in X$. Finally, let us show that $\sum_{n=1}^{N} f_{n} \rightarrow f$ when $N \rightarrow \infty:$

$$
\left\|f-\sum_{n=1}^{N} f_{n}\right\|_{X}=\left\|\sum_{n=N+1}^{\infty} f_{n}\right\|_{X} \leq\left\|\sum_{n=N+1}^{\infty}\left|f_{n}\right|\right\|_{X} \leq \sum_{n=N+1}^{\infty}\left\|f_{n}\right\|_{X} \rightarrow 0 \text {, when } N \rightarrow \infty
$$

Thus, $\sum_{n} f_{n} \in X$ and $X$ is a Banach space.

Lastly, let $f \in X$ and $g \in L_{0}$ be such that $|g| \leq|f| \mu$-a.e.. Then, there are $\lambda>0$ and functions $h_{1} \in B_{X_{1}}, h_{2} \in B_{X_{2}}$ such that

$$
|g| \leq|f| \leq \lambda\left|h_{1}\right|^{1-\theta}\left|h_{2}\right|^{\theta}, \mu \text {-a.e. }
$$

From (4.7) it follows then that $g \in X$ and $\|g\|_{X} \leq\|f\|_{X}$. Therefore, $X$ is a Banach lattice.

Example 4.2.4. If $X_{1}=L_{p}, X_{2}=L_{q}, 1<p, q<\infty$, then $X=X_{1}^{1-\theta} X_{2}^{\theta}=L_{r}$, with $\frac{1}{r}=\frac{1-\theta}{p}+\frac{\theta}{q}$.

Let $f \in X$, then there are functions $g \in B_{L_{p}}, h \in B_{L_{q}}$ and $\lambda>0$ such that

$$
|f| \leq \lambda|g|^{1-\theta}|h|^{\theta}, \mu \text {-a.e. }
$$

Thus, from Hölder's Inequality (Theorem 1.2.2),

$$
\begin{aligned}
\int|f|^{r} d \mu & \leq \lambda^{r} \int|g|^{r(1-\theta)}|h|^{r \theta} d \mu \leq \lambda^{r}\left(\int|g|^{p} d \mu\right)^{\frac{r(1-\theta)}{p}}\left(\int|h|^{q} d \mu\right)^{\frac{r \theta}{q}} \\
& =\lambda^{r}\|g\|_{L_{p}}^{r(1-\theta)}\|h\|_{L_{q}}^{r \theta} \leq \lambda^{r}<\infty
\end{aligned}
$$

Therefore, $f \in L_{r}$ and $\|f\|_{L_{r}} \leq\|f\|_{X}$.

On the other hand, if $f \in L_{r}, f \neq 0$, consider

$$
g=\left(\frac{|f|^{r}}{\|f\|_{L_{r}}}\right)^{\frac{1}{p}}, h=\left(\frac{|f|^{r}}{\|f\|_{L_{r}}}\right)^{\frac{1}{q}} \text { and } \lambda=\|f\|_{L_{r}}>0 .
$$

Note that $g \in B_{L_{p}}$ and $h \in B_{L_{q}}$. Moreover,

$$
\lambda|g|^{1-\theta}|h|^{\theta}=\|f\|_{L_{r}} \frac{|f|^{\frac{r(1-\theta)}{p}}}{\left(\int|f|^{r} d \mu\right)^{\frac{1-\theta}{p}}} \frac{|f|^{\frac{r \theta}{q}}}{\left(\int|f|^{r} d \mu\right)^{\frac{\theta}{q}}}=\|f\|_{L_{r}} \frac{|f|}{\|f\|_{L_{r}}}=|f|
$$


which implies that $f \in X$ and $\|f\|_{X} \leq\|f\|_{L_{r}}$.

Note that in the example above, the result of the Calderón-Lozanovskii construction coincides with the result of the complex interpolation method. We will see that under certain conditions, that is always the case.

As we mentioned above, we want to consider the interpolation of Banach Lattices. With this in mind, note that $X_{1} \cap X_{2}$ and $X_{1}+X_{2}$ are also Banach lattices on $L_{0}$ with the norms defined in Section 2.2.

Indeed, consider $f \in X_{1}+X_{2}$ and $g \in L_{0}$ such that $|g| \leq|f| \mu$-a.e. We have that

$$
\|f\|_{X_{1}+X_{2}}=\inf \left\{\left\|f_{1}\right\|_{X_{1}}+\left\|f_{2}\right\|_{X_{2}}: f=f_{1}+f_{2}, f_{1} \in X_{1}, f_{2} \in X_{2}\right\} .
$$

From the infimum property, for each $\epsilon>0$ there are $f_{1} \in X_{1}, f_{2} \in X_{2}$ such that $f=f_{1}+f_{2}$ and $\left\|f_{1}\right\|_{X_{1}}+\left\|f_{2}\right\|_{X_{2}} \leq\|f\|_{X_{1}+X_{2}}+\epsilon$. Put

$$
\tilde{g}(x)=\left\{\begin{array}{l}
\frac{f_{1}(x) g(x)}{f(x)}+\frac{f_{2}(x) g(x)}{f(x)}, \text { if } f(x) \neq 0 \\
0, \text { if } f(x)=0
\end{array}\right.
$$

Note that $g=\tilde{g} \mu$-a.e. Moreover, for $i=1,2$

$$
\left|\frac{f_{i} g}{f}\right|=\left|\frac{f_{i} g}{f_{1}+f_{2}}\right| \leq\left|\frac{f_{i} f}{f_{1}+f_{2}}\right| \leq\left|f_{i}\right|
$$

Thus,

$$
\left\|\frac{f_{i} g}{f}\right\|_{X_{i}} \leq\left\|f_{i}\right\|_{X_{i}}, i=1,2
$$

and we have

$$
\|g\|_{X_{1}+X_{2}} \leq\left\|\frac{f_{1} g}{f}\right\|_{X_{1}}+\left\|\frac{f_{2} g}{f}\right\|_{X_{2}} \leq\left\|f_{1}\right\|_{X_{1}}+\left\|f_{2}\right\|_{X_{2}} \leq\|f\|_{X_{1}+X_{2}}+\epsilon
$$

Since $\epsilon>0$ is arbitrary, we have that $\|g\|_{X_{1}+X_{2}} \leq\|f\|_{X_{1}+X_{2}}$, which proves that $X_{0}+X_{1}$ is a Banach lattice.

Now, let $f \in X_{1} \cap X_{2}$ and $g \in L_{0}$ with $|g| \leq|f| \mu$-a.e., then, from the order on $X_{1}$ and $X_{2}$

$$
\|g\|_{X_{1} \cap X_{2}}=\max \left\{\|g\|_{X_{1}},\|g\|_{X_{2}}\right\} \leq \max \left\{\|f\|_{X_{1}},\|f\|_{X_{2}}\right\}=\|f\|_{X_{1} \cap X_{2}} .
$$

Thus, $X_{1} \cap X_{2}$ is a Banach lattice.

Moreover, the space $X_{1}^{1-\theta} X_{2}^{\theta}$ is an intermediate space between $X_{1}$ and $X_{2}$. The following theorem gives us conditions for which $X_{1}^{1-\theta} X_{2}^{\theta}$ becomes an interpolation space (and coincides with the result of the complex interpolation space with respect to the compatible couple $\left.\left(X_{1}, X_{2}\right)\right)$.

Theorem 4.2.5. If $X_{1}$ and $X_{2}$ are Banach lattices on $L_{0}$, then for every $0<\theta<1$ it holds that

$$
\left(X_{1}, X_{2}\right)_{\theta}={\overline{X_{1} \cap X_{2}}}^{\left(X_{1}, X_{2}\right)_{\theta}}={\overline{X_{1} \cap X_{2}}}^{X_{1}^{1-\theta} X_{2}^{\theta}}
$$


with equality of norms.

Proof. See [RT10], Theorem 9.

Note that the first equality in the previous theorem follows from Theorem 2.3.6. Furthermore, note we have a condition for the result of the Calderón-Lozanovskii construction and the complex interpolation space to coincide: if $X_{1} \cap X_{2}$ is dense in $X_{1}^{1-\theta} X_{2}^{\theta}$, then

$$
\left(X_{1}, X_{2}\right)_{\theta}={\overline{X_{1} \cap X_{2}}}^{X_{1}^{1-\theta} X_{2}^{\theta}}=X_{1}^{1-\theta} X_{2}^{\theta} .
$$

In particular, the $L_{p}$ spaces satisfy this condition.

\subsection{Banach Lattices and Uniform Homeomorphisms}

Now we want to consider the notion of minimal functions in the Banach lattice setting and find the form of uniform homeomorphisms in this case (in particular, we want to recover the examples of $L^{p}$ spaces for $\left.1<p<\infty\right)$.

Definition 4.3.1. Let $X$ be a Banach lattice on $L_{0}$ and $1<p<\infty$. We define the $p$-convexification of $X$ by

$$
X_{(p)}=\left\{f \in L_{0}:|f|^{p} \in X\right\}
$$

equipped with the norm

$$
\|f\|_{X_{(p)}}=\left\||f|^{p}\right\|_{X}^{1 / p}
$$

Proposition 4.3.2. Consider $X$ a Banach lattice on $L_{0}, 1<p<\infty$ and $\varphi: \Omega \times[0, \infty) \longrightarrow \mathbb{R}$ given by $\varphi(x, t)=t^{\frac{1}{p}}$. Then $\varphi(X)=X_{(p)}$, with equality of norms.

Proof. Let $g \in \varphi(X)$ and $\epsilon>0$. By the infimum property, there are $\lambda \geq 0$ and $f \in B_{X}, f \geq 0$, such that

$$
|g| \leq \lambda|f|^{\frac{1}{p}} \mu \text {-a.e., and } \lambda \leq\|g\|_{\varphi(X)}+\epsilon .
$$

Note that $\lambda^{p}|f| \in X$ and $|g|^{p} \leq \lambda^{p}|f|$. Since $X$ is a Banach lattice, it follows that $|g|^{p} \in X$, which implies that $g \in X_{(p)}$. Moreover, it follows from (4.8) and the definition of Banach lattice that

$$
\|g\|_{X_{(p)}}^{p}=\left\||g|^{p}\right\|_{X} \leq \lambda^{p}\|f\|_{X} \leq \lambda^{p} \leq\left(\|g\|_{\varphi(X)}+\epsilon\right)^{p} .
$$

Thus, since $\epsilon>0$ is arbitrary, we have $\|g\|_{X_{(p)}} \leq\|g\|_{\varphi(X)}$. On the other hand, let $g \in X_{(p)}, g \neq 0$. Let

$$
f=\frac{|g|^{p}}{\|g\|_{X_{(p)}}^{p}} \text { and } \lambda=\|g\|_{X_{(p)}}>0 .
$$

Note that $f \in B_{X}$. Moreover, $\lambda|f|^{\frac{1}{p}}=|g|$. Therefore, $g \in \varphi(X)$ and $\|g\|_{\varphi(X)} \leq\|g\|_{X_{(p)}}$.

It follows from the previous proposition and Theorem 4.2.2 that $X_{(p)}$ is a Banach lattice. 
Let $1<p_{0}<p_{1}<\infty$ and $0<\theta<1$, and consider $X_{\left(p_{0}\right)}$ and $X_{\left(p_{1}\right)}$. Suppose that $X_{\left(p_{0}\right)} \cap X_{\left(p_{1}\right)}$ is dense in $X_{\left(p_{0}\right)}^{1-\theta} \cdot X_{\left(p_{1}\right)}^{\theta}$. Applying the complex interpolation method to this convexifications, from Theorem 4.2 .5 we have that

$$
\left(X_{\left(p_{0}\right)}, X_{\left(p_{1}\right)}\right)_{\theta}=X_{\left(p_{0}\right)}^{1-\theta} \cdot X_{\left(p_{1}\right)}^{\theta}
$$

Moreover, under the assumptions above, we have the following result:

Proposition 4.3.3. $\left(X_{\left(p_{0}\right)}, X_{\left(p_{1}\right)}\right)_{\theta}=X_{\left(p_{\theta}\right)}$, with equal norms, where $\frac{1}{p_{\theta}}=\frac{1-\theta}{p_{0}}+\frac{\theta}{p_{1}}$.

Proof. Let $f \in X_{\left(p_{\theta}\right)}, f \neq 0$. Consider

$$
g=\left(\frac{|f|^{p_{\theta}}}{\|f\|_{X_{\left(p_{\theta}\right)}}^{p_{\theta}}}\right)^{\frac{1}{p_{0}}}, h=\left(\frac{|f|^{p_{\theta}}}{\|f\|_{X_{\left(p_{\theta}\right)}}^{p_{\theta}}}\right)^{\frac{1}{p_{1}}} \text { and } \lambda=\|f\|_{X_{\left(p_{\theta}\right)}}>0 .
$$

Note that $g \in B_{X_{\left(p_{0}\right)}}$ and $h \in B_{X_{\left(p_{1}\right)}}$. Moreover,

$$
\lambda|g|^{1-\theta}|h|^{\theta}=\|f\|_{X_{\left(p_{\theta}\right)}}\left(\frac{|f|^{p_{\theta}}}{\|f\|_{X_{\left(p_{\theta}\right)}}^{p_{\theta}}}\right)^{\frac{1}{p_{\theta}}}=|f|
$$

which implies that $f \in\left(X_{\left(p_{0}\right)}, X_{\left(p_{1}\right)}\right)_{\theta}=X_{\left(p_{0}\right)}^{1-\theta} \cdot X_{\left(p_{1}\right)}^{\theta}$ and $\|f\|_{\left(X_{\left(p_{0}\right)}, X_{\left(p_{1}\right)}\right)_{\theta}} \leq\|f\|_{X_{\left(p_{\theta}\right)}}$.

On the other hand, let $f \in\left(X_{\left(p_{0}\right)}, X_{\left(p_{1}\right)}\right)_{\theta}$ and $\epsilon>0$. Then, there are $\lambda>0, g \in B_{X_{\left(p_{0}\right)}}$ and $h \in B_{X_{\left(p_{1}\right)}}$ such that

$$
|f|^{p_{\theta}} \leq \lambda^{p_{\theta}}|g|^{1-\theta}|h|^{\theta} \mu \text {-a.e. and } \lambda \leq\|f\|_{\left(X_{\left(p_{0}\right)}, X_{\left(p_{1}\right)}\right)_{\theta}}+\epsilon .
$$

Since $f(u)=\exp u$ is a convex function and $\frac{1-\theta}{p_{0}} p_{\theta}+\frac{\theta}{p_{1}} p_{\theta}=1$, we have

$$
\begin{aligned}
|f|^{p_{\theta}} & \leq \lambda^{p_{\theta}}|g|^{(1-\theta) p_{\theta}}|h|^{\theta p_{\theta}}=\lambda^{p_{\theta}}|g|^{\frac{(1-\theta) p_{\theta}}{p_{0}} p_{0}}|h|^{\frac{\theta p_{\theta}}{p_{1}} p_{1}} \\
& =\lambda^{p_{\theta}} \exp \left(\frac{(1-\theta) p_{\theta}}{p_{0}} \ln \left(|g|^{p_{0}}\right)+\frac{\theta p_{\theta}}{p_{1}} \ln \left(|h|^{p_{1}}\right)\right) \\
& \leq \lambda^{p_{\theta}}\left(\frac{(1-\theta) p_{\theta}}{p_{0}}|g|^{p_{0}}+\frac{\theta p_{\theta}}{p_{1}}|h|^{p_{1}}\right)
\end{aligned}
$$

Since $|g|^{p_{0}},|h|^{p_{1}} \in X$ and $X$ is a Banach lattice, it follows that $|f|^{p_{\theta}} \in X$, that is, $f \in X_{\left(p_{\theta}\right)}$. Furthermore, from (4.9) we have that

$$
\begin{aligned}
\|f\|_{X_{\left(p_{\theta}\right)}}^{p_{\theta}} & =\left\||f|^{p_{\theta}}\right\|_{X} \leq \lambda^{p_{\theta}}\left(\frac{(1-\theta) p_{\theta}}{p_{0}} p_{0}\left\||g|^{p_{0}}\right\|_{X}+\frac{\theta p_{\theta}}{p_{1}} p_{1}\left\||h|^{p_{1}}\right\|_{X}\right) \\
& =\lambda^{p_{\theta}}\left(\frac{(1-\theta) p_{\theta}}{p_{0}}\|g\|_{X_{\left(p_{0}\right)}}^{p_{0}}+\frac{\theta p_{\theta}}{p_{1}}\|h\|_{X_{\left(p_{1}\right)}}^{p_{1}}\right) \leq \lambda^{p_{\theta}} \leq\left(\|f\|_{\left(X_{\left(p_{0}\right)}, X_{\left(p_{1}\right)}\right)_{\theta}}+\epsilon\right)^{p_{\theta}}
\end{aligned}
$$

therefore, $\|f\|_{X_{\left(p_{\theta}\right)}} \leq\|f\|_{\left(X_{\left(p_{0}\right)}, X_{\left(p_{1}\right)}\right)_{\theta}}$.

Again assuming the above hypotheses, we can consider the Calderón-Lozanovskii decomposition of 
a function

$$
f \in\left(X_{\left(p_{0}\right)}, X_{\left(p_{1}\right)}\right)_{\theta}=X_{\left(p_{0}\right)}^{1-\theta} \cdot X_{\left(p_{1}\right)}^{\theta}
$$

given by

$$
f=\operatorname{sgn}(f) g^{1-\theta} h^{\theta}
$$

where $\frac{1}{p_{\theta}}=\frac{1-\theta}{p_{0}}+\frac{\theta}{p_{1}}, g=|f|^{p_{\theta} / p_{0}}$ and $h=|f|^{p_{\theta} / p_{1}}$.

Theorem 4.3.4. Let $1<p_{0}<p_{1}<\infty$ and $0<\theta<1$. The minimal lifting map $\Gamma: S_{X_{\left(p_{0}\right)}^{1-\theta} \cdot X_{\left(p_{1}\right)}^{\theta}} \longrightarrow$ $\mathcal{F}_{\theta}^{p}(\bar{A})$ is given by

$$
\Gamma(f)(z)=\operatorname{sgn}(f) g^{1-z} h^{z}
$$

for each $f \in S_{X_{\left(p_{0}\right)}^{1-\theta} \cdot X_{\left(p_{1}\right)}^{\theta}}$ and $z \in \overline{\mathbb{S}}$, where $\frac{1}{p_{\theta}}=\frac{1-\theta}{p_{0}}+\frac{\theta}{p_{1}}, g=|f|^{p_{\theta} / p_{0}}$ and $h=|f|^{p_{\theta} / p_{1}}$.

Furthermore, according to Theorem 3.2.5, we have the following corollary:

Corollary 4.3.5. Let $1<p_{0}<p_{1}<\infty$ and $0<\theta_{1}, \theta_{2}<1$. The mapping

$$
\begin{aligned}
U: S_{X_{\left(p_{0}\right)}^{1-\theta_{1}} \cdot X_{\left(p_{1}\right)}^{\theta_{1}}} & \longrightarrow S_{X_{\left(p_{0}\right)}^{1-\theta_{2}} \cdot X_{\left(p_{1}\right)}^{\theta_{2}}} \\
f & \longmapsto \operatorname{sgn}(f)|f|^{\frac{p_{1}}{\theta_{2}}}
\end{aligned}
$$

defines a uniform homeomorphism, where $\frac{1}{p_{\theta_{1}}}=\frac{1-\theta_{1}}{p_{0}}+\frac{\theta_{1}}{p_{1}}$ and $\frac{1}{p_{\theta_{2}}}=\frac{1-\theta_{2}}{p_{0}}+\frac{\theta_{2}}{p_{1}}$.

Note that the mapping $U$ from the previous corollary is exactly the uniform homeomorphism given between the unit sphere of the spaces $L_{p_{\theta_{1}}}$ and $L_{p_{\theta_{2}}}$ as we saw in Section 3.3. 



\section{Chapter 5}

\section{Further discussion}

A major object of the theory involving complex interpolation is the study of quasi-Banach spaces.

If $X$ is a vector space over $\mathbb{K}$ (where $\mathbb{K}=\mathbb{R}$ or $\mathbb{C}$ ), we say that $\|\cdot\|: X \rightarrow[0, \infty)$ is a quasi-norm on $X$ if

(i) $\|x\|=0$ if and only if $x=0$;

(ii) $\|\alpha x\|=|\alpha|\|x\|$, if $\alpha \in \mathbb{K}$ and $x \in X$.

(iii) Exists a constant $C \geq 1$ such that if $x_{1}, x_{2} \in X$, then $\left\|x_{1}+x_{2}\right\| \leq C\left(\left\|x_{1}\right\|+\left\|x_{2}\right\|\right)$.

The Aoki-Rolewicz Theorem states that if $0<p \leq 1$ and $C=2^{\frac{1}{p}-1}$, then there is a constant $B$ such that if $x_{1}, \cdots, x_{m} \in X$ we have

$$
\left\|\sum_{n=1}^{m} x_{n}\right\| \leq B\left(\sum_{n=1}^{m}\left\|x_{n}\right\|^{p}\right)^{\frac{1}{p}} .
$$

It is then possible to replace $\|\cdot\|$ by an equivalent $p$-subadditive quasi-norm $\|\cdot\|$ so that

$$
\left\|x_{1}+x_{2}\right\| \leq\left(\left\|x_{1}\right\|^{p}+\left\|x_{2}\right\|^{p}\right)^{1 / p} .
$$

The quasi-norm $\|\cdot\|$ induces a metric topology on $X ; X$ is called a quasi-Banach space if $X$ is complete for this metric.

It is possible to consider the construction of Calderón-Lozanovskii (as seen in Section 4.2.1), however considering the quasi-Banach lattice setting as follows: Given $\left(X_{0}, X_{1}\right)$ a compatible pair of quasiBanach lattices and a function $\varphi: \mathbb{R}_{+}^{2} \rightarrow \mathbb{R}_{+}$which is homogeneous and non-decreasing in each argument, the space $\varphi\left(X_{0}, X_{1}\right)$ is given by $x \in X_{0}+X_{1}$ such that $|x| \leq \varphi\left(x_{0}, x_{1}\right)$ for some $x_{0} \in X_{0}$ and $x_{1} \in X_{1}$. The space becomes a quasi-Banach lattice when endowed with the quasi-norm

$$
\|x\|_{\varphi\left(X_{0}, X_{1}\right)}=\inf \left\{\lambda>0:|x| \leq \lambda \varphi\left(x_{0}, x_{1}\right),\left\|x_{0}\right\|_{X_{0}} \leq 1,\left\|x_{1}\right\|_{X_{1}} \leq 1\right\} .
$$

In [RT18] the authors study the interpolation properties of the Calderón-Lozanovskii construction in the quasi-Banach lattice setting. It remains open what would be the concepts of minimal functions in this setting. 



\section{Bibliography}

[Ahl66] L. V. Ahlfors. Complex analysis: an introduction to the theory of analytic functions of one complex variable, volume 2. McGraw-Hill New York, 1966. 17, 18, 55

[AL85] I. Aharoni e J. Lindenstrauss. An extension of a result of Ribe. Israel Journal of Mathematics, 52:59-64, 1985. 1

[Bea11] B. Beauzamy. Introduction to Banach spaces and their geometry. Elsevier, 2011. 7, 8

[BL12] J. Bergh e J. Löfström. Interpolation spaces: an introduction, volume 223. Springer Science \& Business Media, 2012. 29, 37, 41

[BS88] Colin Bennett e Robert C Sharpley. Interpolation of operators. Academic press, 1988. 29

[Cal64] A. Calderón. Intermediate spaces and interpolation, the complex method. Studia Mathematica, 24(2):113-190, 1964. 2, 29, 37, 50, 83, 84

[CCR $\left.{ }^{+} 82\right]$ R. R. Coifman, M. Cwikel, R. Rochberg, Y. Sagher e G. Weiss. A theory of complex interpolation for families of Banach spaces. Advances in Mathematics, 43(3):203-229, 1982. 50

[CR82] M. Cwikel e S. Reisner. Interpolation of uniformly convex banach spaces. Proceedings of the American Mathematical Society, 84(4):555-559, 1982. 70

[Dah95] M. Daher. Homéomorphismes uniformes entre les sphères unité des espaces d'interpolation. Canadian Mathematical Bulletin, 38(3):286-294, 1995. 2, 5, 51, 52, 53

[DUJ43] J. Diestel e J. J. Uhl Jr. Vector measures, volume 15 of American Mathematical Society. Mathematical Surveys. Library of Congress Cataloging in Publication Data, 1943. 21

[Dur70] P. L. Duren. Theory of Hp Spaces. Pure and applied mathematics / H. Bass and S. Eilenberg editors. Academic Press, 1970. 15, 16, 17

[Enf70a] P. Enflo. On the nonexistence of uniform homeomorphisms between Lp-spaces. Arkiv för matematik, 8(2):103-105, 1970. 1

[Enf70b] P. Enflo. Uniform structures and square roots in topological groups, part ii. Israel Journal of Mathematics, 8:253-272, 1970. 1

[FHH $\left.{ }^{+} 01\right]$ M. Fabian, P. Habala, P. Hájek, V. M Santalucía, J. Pelant e V. Zizler. Functional analysis and infinite-dimensional geometry. Springer Science \& Business Media, 2001. 5

[Fol99] G. B. Folland. Real analysis: modern techniques and their applications, volume 40. John Wiley \& Sons, 1999. 10, 12 
[GP77] J. Gustavsson e J. Peetre. Interpolation of Orlicz spaces. Studia Mathematica, 60(1):3359, 1977. 50

[Har15] G. H. Hardy. The mean value of the modulus of an analytic function. Proceedings of the London Mathematical Society, 2(1):269-277, 1915. 15

[Her86] E. Hernández. Intermediate spaces and the complex method of interpolation for families of Banach spaces. Annali della Scuola Normale Superiore di Pisa-Classe di Scienze, 13(2):245-266, 1986. 50

[JLS96] W. B. Johson, J. Lindenstrauss e G. Schechtman. Banach spaces determined by their uniform structures. Geometric and Functional Analysis GAFA, 6:430-470, 1996. 1

[Kad67] M. I. Kadec. Proof of the topological equivalence of all separable infinite-dimensional Banach spaces. Functional Analysis and Its applications, 1(1):53-62, 1967. 1

[Kal13] N. J. Kalton. Examples of uniformly homeomorphic Banach spaces. Israel Journal of Mathematics, 194:151-182, 2013. 1

[Koo98] P. Koosis. Introduction to Hp spaces, volume 115. Cambridge University Press, 1998. 15

[KS02] S. G. Krein e E. M. Semenov. Interpolation of linear operators, volume 54. American Mathematical Soc., 2002. 29

[Lim87] B. V. Limaye. Banach space-valued analytic functions. Em Spectral perturbation and approximation with numerical experiements, páginas 44-60, Canberra AUS, 1987. Centre for Mathematical Analysis, The Australian National University. 17

[Lin64] J. Lindenstrauss. On nonlinear projections in Banach spaces. The Michigan Mathematical Journal, 11(3):263-287, 1964. 1

[Lun18] Alessandra Lunardi. Interpolation theory, volume 16. Springer, 2018. 29

[Maz29] S. Mazur. Une remarque sur l'homéomorphie des champs fonctionnels. Studia Mathematica, 1(1):83-85, 1929. 1, 70

[Meg12] R. E. Megginson. An introduction to Banach space theory, volume 183. Springer Science \& Business Media, 2012. 5, 6, 7, 8, 14

[Mik78] J. Mikusiński. The Bochner Integral. Springer, 1978. 21

[Phe60] R. R. Phelps. Uniqueness of Hahn-Banach extensions and unique best approximation. Transactions of the American Mathematical Society, 95(2):238-255, 1960. 7

[Phi43] R. S. Phillips. On weakly compact subsets of a Banach space. American Journal of Mathematics, 65(1):108-136, 1943. 25

[Pis16] G. Pisier. Martingales in Banach spaces, volume 155. Cambridge University Press, 2016. $15,17,27$

[Rem98] R. Remmert. Theory of Complex Functions, volume 122 of Graduate Texts in Mathematics. Springer Science \& Business Media, 1998. 17, 18

[Rib84] M. Ribe. Existence of separable uniformly homeomorphic nonisomorphic Banach spaces. Israel Journal of Mathematics, 48:139-147, 1984. 1

[Rie23] F. Riesz. Über die randwerte einer analytischen funktion. Mathematische Zeitschrift, 18(1):87-95, 1923. 15 
[RS12] M. Reed e B. Simon. Methods of Modern Mathematical Physics: Functional Analysis. Elsevier, 2012. 17

[RT10] Y. Raynaud e P. Tradacete. Interpolation of Banach lattices and factorization of pconvex and q-concave operators. Integral Equations and Operator Theory, 66(1):79-112, 2010. 91

[RT18] Y. Raynaud e P. Tradacete. Calderón-Lozanovskii interpolation on quasi-Banach lattices. Banach Journal of Mathematical Analysis, 12(2):294-313, 2018. 95

[Rud70] W. Rudin. Real and Complex Analysis. Tata McGraw-hill education, 1970. 10, 11, 15

[Wid61] D. V. Widder. Functions harmonic in a strip. Proceedings of the American Mathematical Society, 12(1):67-72, 1961. 51 


\section{Index}

$A_{\theta}^{p}, 52$

$A_{\theta}, 39$

$B\left(x_{0}, r\right), 5$

$B_{X}, 5$

$B_{z_{0}}^{p}, 53$

Banach lattice, 83

Blaschke product, 16

Bochner integrable, 21

Bochner measurable, 21

Calderón-Lozanovskii construction, 87 canonical factorization theorem, 16

Cauchy's integral formula, 17

Cauchy's Integral Theorem, 18

compatible couple, 33

complex interpolation space, 39

complex logarithm, 54

concave function, 84

conjugate exponent, 11

$\mathcal{F}(\bar{A}), 38$

$\mathcal{F}_{\theta}^{p}(\bar{A}), 52$

Fatou's theorem, 15

$\mathcal{H}(\bar{A}), 52$

$\mathcal{H}_{z_{0}}^{p}(\bar{A}), 53$

Hölder's Inequality, 11

Hahn-Banach Theorem, 6

Hardy space, 15

Hille's theorem, 22

holomorphic function, 20

strongly, 19

weakly, 19

$L_{0}, 83$

$L_{p}$ space, 11

Lebesgue's Dominated Convergence Theorem, 10

Maximum Modulus Principle, 18

Mazup map, 79

Mazur map, 70

measure

absolutely continuous, 11 minimal function, 68

minimal lifting map, 68

modulus of convexity, 7

Morera's Theorem, 18

Orlicz space, 50

outer function, 16

$p$-convexification, 91

$p$-direct sum, 8

Phragmén-Lindelöf Maximum Principle, 18

Phragmen-Lindelöff Maximum Principle for Banach spaces, 21

Poisson Integral, 15

Poisson kernel, 15, 52

radial limit, 15

Radon-Nikodým Theorem, 11

reflexive space, 6

Riesz-Thorin Interpolation Theorem, 30

$S_{X}, 5$

singular inner function, 16

Sobolev space, 50

stricly convex space, 7

Three Line theorem, 30

topological vector space, 33

uniformly convex space, 8

vector valued $L_{p}$ space, 24

vertical maps, 77

weighted $L_{p}$ space, 14

$Y \hookrightarrow X, 33$ 